\title{
REFERENCE
}

\section{Workshop on IEEE-1588, Standard for a Precision Clock Synchronization Protocol for Networked Measurement and Control Systems}

Lee, K. B.

U. S. DEPARTMENT OF COMMERCE Technology Administration National Institute of Standards and Technology

Gaithersburg, MD 20899

\section{QC \\ 100 \\ .456 \\ * 7070 \\ 2003}

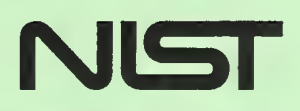

National Instifute of Standards and Technology

Technology Administration

U.S. Department of Commerce 



\section{Workshop on IEEE-1588, Standard for a Precision Clock Synchronization Protocol for Networked Measurement and Control Systems}

Lee, K. B.

U. S. DEPARTMENT OF COMMERCE Technology Administration National Institute of Standards and Technology

Gaithersburg, MD 20899

November 20, 2003

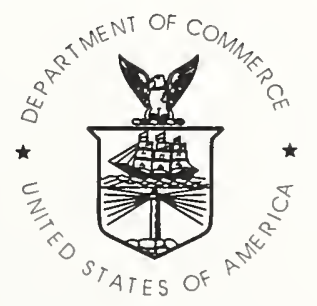

U.S. DEPARTMENT OF COMMERCE Donald L. Evans, Secretary

TECHNOLOGY ADMINISTRATION

Phillip J. Bond, Under Secretary for Technology

NATIONAL INSTITUTE OF STANDARDS

AND TECHNOLOGY

Arden L. Bement, Jr., Director 
NISTIR 7070

\section{Workshop on IEEE-1588, Standard for a Precision Clock Synchronization Protocol for Networked Measurement and Control Systems}

Kang Lee

National Institute of Standards and Technology

Gaithersburg, Maryland

John Eidson

Agilent Technologies

Palo Alto, California

September 24, 2003

DISCLAIMER:

This publication consists of workshop proceedings containing presentation slides, technical papers, recommendations. and other materials contributed by participants of this workshop. This publication provides the material as presented and discussed at the workshop in its original form, without modification by the National Institute of Standards and Technology (NIST). Commercial equipment and software referred to in this document are identified for informational purposes only, and does not imply recommendation of or endorsement by NIST, nor does it imply that the products so identified are the best available for the purpose. 


\section{TABLE OF CONTENTS}

Executive Summary

Summary of Points Noted During the Open Discussion Section..................................................... 5

Agenda.

Time Synchronization in Switched Ethernet

Øyvind Holmeide, OnTime Networks AS

Boundary Clock Implementation with Time Synchronization Protocol Analyzer 20

Embedded SynUTC and IEEE 1588 Clock Synchronization for

Industrial Ethernet 26

Nikolaus E. Kerö, Oregano Systems, Hannes Muhr, Georg Gaderer, Roland Höller,

Thilo Sauter, Institute of Computer Technology, Vienna University of Technology,

Horaver Martin, Teehnikun Wien

PTP in Redundant Network Structures 38

Ludwig Winkel, Siemens Automation and Drives

A Solution for Fault-Tolerant IEEE-1588 (Slides)

A Solution for Fault-Tolerant IEEE-1588 (Paper)

Jeff Allan, Dongik Lee, Dependable Real-Time Systems Ltd.

PTP in Switched Networks

Thomas Mueller, Zurieh University of Applied Seienec, Winterthur, Suisse,

Karl Weber, Siemens Automation and Drives

Impact of Switch Cascading on Time Accuracy

IEEE 1588 Network Devices

Dirk S. Mohl, Hirshmann Eleetronies

A Frequency Compensated Clock for Precision Synchronization

using IEEE 1588 Protocol and its Application to Ethernet

Sivaran Balasubramanian, Kendal R. Harris and Anatoly Moldovansky,

Roekwell Automation

IEEE-1588 Node Synchronization Improvement by High Stability Oscillators

John C. Eidson, Bruce Hamilton, Agilent Technologies

IEEE $1588^{\mathrm{TM}}$ Node Synchronization Improvement by High Stability Oscillators

Time Correlation on a Network Based Airborne Telemetry System

Jiwang Dai, Edward Grozalis, L3 Communications Telemetry East, Thomas DeSelms, Veridian

Enginecring

Implementation of IEEE Std.-1588 in a Networked I/O Node

Mark E. Shepard, Douglas G. Fowley, Roy L. Jackson, Dennis B. King,

GE Drives \& Controls, Ine.

Application of IEEE 1588 to a Distributed Motion Control System

Kendal Harris, Sivaram Balasubramanian, Anatoly Moldovansky

Rockwell Automation

IEEE 1588 Proposal for Metro Ethernet Enterprise Solutions

Glenn Algie, Nortel Networks

Appendix A

Question and Answers on Workshop Papers

Demonstrations:

- IEEE 1588 Prototype Demonstration, Hirshmann Eleetronies

- Demonstration for the Application of the IEEE 1588 to Distributed Motion Control, Rockwell Automation

- Implementation of IEEE Std.-1588 in a Networked I/O Node, GE Drives \& Controls (no slide provided)

- Set up to Show Effeets of Oseillator Quality on Synehronization, Agilent Teehnologies

- Boundary Clock Implementation, On Time Networks (no slide provided)

- Extending IEEE 1588 to fault tolerant synehronization with a worst-case preeision in the $100 \mathrm{~ns}$ range, Oregano Systems (no slide provided)

Final Participants List 


\section{Workshop Summary}

\section{Executive Summary from the Conference Co-Chairs}

The IEEE 1588 standard defines a protocol enabling precise synchronization of clocks in measurement and control systems implemented with technologies such as network communication, local computing, and distributed objects. The protocol enables heterogeneous systems that include clocks of various inherent precision, resolution, and stability to synchronize. The IEEE 1588 standard was approved as ANSI/IEEE 1588-2002 in January 2003. This conference was to provide a forum for users and implementers of the 1588 standard to exchange information and ideas about the standard.

The conference participants represented different sectors of industry ranging from networked instrumentation and measurement, to utility power generation and distribution, to aerospace and avionics, to semiconductor production equipment manufacturing, to telecommunication. The workshop opened with welcoming remarks and a presentation about the National Institute of Standards and Technology (NIST) and the various programs of its Manufacturing Engineering Laboratory (MEL) by the MEL Director Dr. Dale Hall. Dr. Hall pointed out that a workshop like this is one of the many avenues for industry and NIST to work together on standards and technology issues.

Twelve presentations on implementations based on the IEEE 1588 standard or technologies addressing time synchronization issues were presented in three sessions. Following that, a demonstration session was held featuring implementations based on IEEE 1588 from six companies. The demonstrations ranged from networked time synchronization of sensor data acquisition, to motion control, to software tools for time synchronization diagnostics. The final session was an open forum discussion. It focused on issues about the standard identified by the attendees. Attendees were invited to put issues on the board, or add a check mark to existing issue(s) they wanted to discuss. The moderator chose the issues with the most check marks first. The purpose was to get the issues aired and defined and to identify the hot items, not to solve technical issues. At the end, all issues on the board were addressed or at least briefly discussed. The summary of the discussion on these issues is presented below. The ideas and issues brought out in this workshop will be the used to formulate the agenda for the next workshop to be held in the same time frame in 2004. The proceedings for the workshop will be put on the IEEE 1588 Web site at http://cee 1588 .nist.gov as soon as it is approved for publication by NIST. Plans for the next workshop will be presented in the spring of 2004. 


\section{Summary of Points Discussed During the Open Discussion Session}

\section{Market Acceptance / Requirements}

IEEE 1588 is applicable to a much wider range of applications than networking sensors and networked measurement and control systems. Because of its potentially wide application space, many questions still need to be answered in future workshops. They are:

- Which set of markets is appropriate to use the IEEE 1588 standard?

- Once the markets are identified, we must determine for each market

- what set of requirements is appropriate?

- what level of synchronization?

- how much redundancy?

If we don't have a good set of requirements and use cases, we won't be able to make good choices. For example, in the telecommunication space, we may be able to find customers who have advanced technology shops and are willing to help.

Telecommunication requirements are likely to be different from most others. Ideally it would be the same standard, just a different implementation. Applications where IEEE 1588 overlaps other technologies, such as Network Time Protocol (NTP), will need to be examined.

Tools would help acceptance, for example, for a Field Programmable Gate Array (FPGA) core, or for a source code for the protocol stack. It was noted that Application Specific Integrated Circuit (ASIC)/FPGA cores tend to be very application-specific, whereas, the stack and management message handling would be widely applicable. For the FPGA, people could publish the state diagram. In other standards, a marketing track was formed to deal with this issue.

The standard leaves some things to the implementers. We may need more clarification or statements of preferred implementation to assure interoperation. It was noted that the committee keeps questions and interpretations of the standard on the IEEE 1588 Web site. Questions currently go to the IEEE, or committee chair.

\section{Conformance \& Certification}

- Minimum subset for conformance to the IEEE 1588 standard.

- Certification tools/procedures are needed.

- A test set.

- A certification body, or an accepted set of procedures.

- Part of the issues is who has liability if things do not work. The Distributed Network Protocol (http://www.dnp.org) is a nonprofit organization, thus not willing to assume liability. They developed a book saying, "this is how you certify." Anyone who wants to become a certifier pays a license fee for the logo, follows the book, and is a certifier. If something goes wrong, liability falls to the certifier.

- A university, a company, or NIST may become a certifier for a test fee.

- A reference implementation.

- Keen interest in a Plugfest - plug-and-play tests for interoperability.

- Financial support for some of these activities. 


\section{Extensions}

- Ways to update the standard were briefly discussed. Suggested modifications can be partitioned into two categories. One category of modifications fit within the existing standard and the other causes a dramatic modification of the core of the standard. Changes should probably go hand-in-hand with chosen markets.

- Additional payload (in existing packets, or extension packets)

- IPv6

- 802.3 tagged frames (may be able to adopt this \& Ipv6 as alternate profiles).

- "Bare" Ethernet, below Internet Protocol (IP) and Unified Data Protocol (UDP), e.g., Ethernet-level multicast. Some embedded systems don't use TCP or UDP. On the other hand, it's easier to use off-the-shelf systems when your packets have IP headers. The IP join/leave mechanism may be useful for management.

- Redundancy. Part of this issue includes defining what it means.

- Bypass clocks.

- Networks other than LAN, e.g., physically bigger or poorer-quality links.

- Support for heterogeneous components, along the lines of Simple Network Time Protocol (SNTP).

- What is the governing body? IEEE has rules for amending a standard, but they address only procedures, not content. A Project Authorization Request (PAR) needs to be formulated and submitted to IEEE-SA in order to obtain a project to update the standard.

- Task groups are needed to hold technical discussions and coordinate the work on the standard. They will be formed shortly after the workshop. In the group discussion, we will prioritize the tasks and get a clear view of the requirements before opening any PAR because a PAR carries a deadline.

- Volunteers are solicited to serve in the task groups. Anyone interested to participate should contact John Eidson and Kang Lee.

\section{Conference Proceedings}

When the conference proceedings are ready, they will be available for access on the IEEE 1588 website located at URL: htw:/heeel 588 nist.gov.

\section{Future Workshop}

The plan for future workshop was briefly discussed. Most attendees wanted to have another workshop organized by NIST. Location is to be determined. Practically no one voted for a workshop in six months, but the majority of the attendees preferred to have the next workshop in about one year. Companies participated in the demonstration at this workshop and other attendees expressed interest in showing their implementations based on IEEE 1588 in the next workshop. In addition, participants wanted to have a tutorial session on the detail of the standard - what it is, why is it needed, and how it can be used, etc.

The topics of the next workshop should include:

- Tutorial. There was a specific request for a session on comparing PTP with other clock synchronization protocols.

- Technical papers.

- Reports by the task groups.

- A Plugfest. 
Workshop on

IEEE-1588, Standard for a Precision Clock Synchronization Protocol for Networked Measurement and Control Systems

\author{
Co-sponsored by \\ NIST and IEEE Instrumentation and Measurement Society
}

\author{
NIST \\ Gaithersburg, Maryland \\ September 24, 2003 \\ Workshop: Lecture room A \\ Demonstration: Lecture room B
}

\title{
AGENDA
}

- 8:00 AM: Bus leaves conference hotel for NIST facility

- 8:30-9:00 AM: Continental breakfast, meet other attendees, pick up conference badges and material.

- 9:00-9:15 AM: Workshóp opening Moderator: Kang Lee, NIST

- Welcome from Dr. Dale Hall, Director of the NIST Manufacturing Engineering Laboratory

- Administrative details

- 9:15 AM to 10:35 AM: Technical paper presentations session 1 Moderator: John Eidson, Agilent Technologies

- 9:15-9:35 AM: Boundary Clock implementation: Øyvind Holmeide, Managing Director, OnTime Networks AS.

- 9:35-9:55AM: Extending IEEE 1588 to fault tolerant synchronization with a worst-case precision in the $\mathbf{1 0 0} \mathbf{n s}$ range: Nikoaus E. Keroe, Oregano Systems, Georg Gaderer, Roland Höller and Thilo Sauter, Institute of Computer Technology, Vienna University of Technology

- 9:55-10:15AM: Consequences of Redundant Structures PTP: Ludwig Winkel, SIEMENS Automation and Drives

- 10:15-10:35AM: A Solution for Fault-Tolerant IEEE-1588: Jeff Allan \& Dr Dongik Lee, Dependable Real Time Systems Ltd., Sheffield, U.K.

- 10:35 - 10:50 AM: Morning coffee break

- 10:50 AM-12:10 PM: Technical paper presentations session 2

Moderator: Kang Lee, NIST 
- 10:50-11:10AM: Impact of Switch Cascading on Time Accuracy: Prof. Thomas Mueller, University Wintherthur, Suisse, Karl Weber, SIEMENS Automation and Drives

- 11:10-11:30AM: IEEE 1588 and Network Devices: Dirk S. Mohl, Hirschmann Electronics

- 11:30-11:50AM: A Frequency Compensated Clock for Precision Synchronization using IEEE 1588 Protocol and its Application to Ethernet: Sivaram Balasubramanian, Kendal R. Harris and Anatoly Moldovansky, Rockwell Automation

- 11:50AM-12:10PM: IEEE-1588 Node Synchronization Improvement by High Stability Oscillators: John C Eidson \& Bruce Hamilton, Agilent Laboratories

- 12:10-1:10 PM: Lunch at NIST cafeteria

- 1:10-2:30 PM: Technical paper presentations session 3

Moderator: Joe White, US Naval Research Laboratory

- 1:10-1:30PM: Time Correlation using network based data acquisition on-

board a Military Test Vehicle: Jiwang Dai, Ph.D, Senior Software Engineer, L3

Communications Telemetry East, Thomas DeSelms, Senior Network Systems

Engineer, Veridian Engineering, and Edward Grozalis, Senior Engineer, L3

Communications Telemetry East

- 1:30-1:50PM: Implementation of IEEE Std.-1588 in a Networked I/O Node:

Mark Shepard, GE Drives \& Controls, Inc., Salem, VA

- 1:50-2:10PM: Application of IEEE 1588 to Distributed Motion Control: Kendal

R. Harris, Sivaram Balasubramanian, and Anatoly Moldovansky, Rockwell

Automation

- 2:10-2:30PM: Proposal for IEEE1588 use over Metro Ethernet Layer 2 VPNs:

Glenn Algie, Senior Advisor, Wireless Technology Labs, Nortel Networks

- 2:30-3:30 PM: Demonstration of implementations

Moderator: John Eidson, Agilent Technologies

- OnTime Networks AS, Øyvind Holmeide, Managing Director

- Oregano Systems, Nikoaus E. Keroe

- Hirschmann Electronics, Dirk Mohl \& Dominik Iadonisi

- Rockwell Automation, Kendal R. Harris and Sivaram Balasubramanian

- GE Drives \& Controls, Inc., Salem, VA, Mark Shepard

- Agilent Laboratories, John C Eidson \& Bruce Hamilton

- 3:30-3:45 PM: Afternoon refreshment break

- 3:45-4:45 PM: Open discussion session- Moderator: John Eidson, Scribe: Bruce Hamilton, Agilent Technologies

- Topics selected by attendees

- Next steps

- 4:45-5 PM: Closing comments- Kang Lee \& John Eidson

- 5:15 PM: Bus leaves NIST for conference hotel 


\section{ABSTRACTS FOR TECHNICAL SESSIONS}

Session 1, 9:15 AM to 10:35 AM:

- Boundary Clock implementation: Øyvind Holmeide, Managing Director, OnTime Networks AS. Abstract: The presentation will cover special properties of a boundary clock implementation. The principles for achieving the same timing accuracy on a 1588 boundary clock implementation without Follow_Up packet support as with the support of this feature will also be described.

- Extending IEEE 1588 to fault tolerant synchronization with a worst-case precision in the 100 ns range: Nikoaus E. Keroe, Oregano Systems, Georg Gaderer, Roland Höller and Thilo Sauter, Institute of Computer Technology, Vienna University of Technology Abstract: We present the SynUTC paradigm, fitted into the IEEE1588 standard for realizing the basic functionality in terms of time distribution and accuracy. By using the extended features provided by the SynUTC technology a worst case precision of less than 100 ns under any network load can be realized.

- Consequences of Redundant Structures PTP: Ludwig Winkel, SIEMENS Automation and Drives Abstract: A switch over in redundant structures will result in a loss of delay time when a link fails. A UDP based node cannot detect this failure. A method for handling time synchronization in redundant structures will be proposed.

- A Solution for Fault -Tolerant IEEE-1588: Jeff Allan \& Dr Dongik Lee, Dependable Real Time Systems Ltd., Sheffield, U.K. Abstract: The IEEE-1588 Standard offers a very stable and accurate platform for distributed time-based communications. A claimed weakness however of the current IEEE-1588 Standard relates to a lack of fault-tolerance. Dependable Real Time Systems Ltd (DRTS Ltd) have been developing reliable and faulttolerant clock synchronization techniques for CAN networks. The core technology enables time triggered CAN communications that can be implemented in standard CAN nodes using no extra hardware and needing no new silicon to provide a cost-effective solution for safety-critical applications. This paper outlines how DRTS ideas previously implemented in CAN can be applied to the IEEE-1588 Standard and outlines a simple way in which the current standard could be improved to include a reliable fault-tolerant capability.

Session 2, 10:50 AM-12:10 PM:

- Impact of Switch Cascading on Time Accuracy: Prof. Thomas Mueller, University Wintherthur, Suisse, Karl Weber, SIEMENS Automation and Drives Abstract: Switches add a non-deterministic delay to messages. They may be treated as boundary clocks but the cascading of control loops in each node will introduce additional sources for time inaccuracies. A method for efficient handling of switched networks based on PTP will be proposed.

- IEEE 1588 and Network Devices: Dirk S. Mohl, Hirschmann Electronics Abstract: The presentation will start with an overview of our IEEE 1588 implementation. It will give an overview of the system design (software and hardware) and also show some issues of a portable IEEE1 588 code (Linux, Windows, VxWorks). It will give some details about precision of the implementation and show some ideas for possible improvements in the software stack. The presentation will give some tips on how to implement IEEE 1588 and where to get the necessary software stacks. It will then show why IEEE 1588 is also 
necessary and useful in layer 2 switches. The presentation will close with an overview of possible enhancements for IEEE 1588 like calculation and adjusting clock drifts and using SNMP for management.

- A Frequency Compensated Clock for Precision Synchronization using IEEE 1588 Protocol and its Application to Ethernet: Sivaram Balasubramanian, Kendal R. Harris and Anatoly Moldovansky, Rockwell Automation Abstract: In a distributed control system containing multiple clocks, individual clocks tend to drift apart due to instabilities inherent in source oscillators and environmental conditions such as temperature, mechanical, aging etc. Hence, some kind of correction is necessary to synchronize individual clocks to maintain the notion of global time, which is accurate to some requisite clock resolution. In this paper, we present a frequency compensated clock to achieve precision synchronization amongst distributed clocks using IEEE 1588 protocol to exchange timing information. Further, we explore its application to Ethernet.

- IEEE-1588 Node Synchronization Improvement by High Stability Oscillators: John C Eidson \& Bruce Hamilton, Agilent Laboratories Abstract: This paper outlines work done for the US Naval Research Laboratory to investigate the sensitivity of synchronization accuracy with the stability of local oscillators. The results indicate the likely accuracy obtainable in a variety of configurations.

Session 3, 1:10-2:30 PM:

- Time Correlation using network based data acquisition on-board a Military Test Vehicle: Jiwang Dai, Ph.D, Senior Software Engineer, L3 Communications Telemetry East, Thomas DeSelms, Senior Network Systems Engineer, Veridian Engineering, and Edward Grozalis, Senior Engineer, L3 Communications Telemetry East Abstract: Military avionics busses and processors are getting faster, plus data acquisition systems are specified as needing an order of magnitude better time correlation than the System Under Test (SUT). In combination with these is the drive to lower cost, increase capability and use COTS equipment the test vehicle community is moving to a network based data acquisition system. Using a network based data acquisition system presents problems when attempting to correlate data on a asynchronous network. This paper will detail timing correlation issues when using a network based data acquisition system on-board a military test vehicle and propose IEEE 1588 as one of the solutions.

- Implementation of IEEE Std.-1588 in a Networked I/O Node: Mark Shepard, GE Drives \& Controls, Inc., Salem, VA Abstract: IEEE Std. 1588-2002 is very specific in its description of the Precision Time Protocol for exchanging information between clocks. However, many of the detailed algorithms for disciplining, converging and tracking the actual clocks are "beyond the scope" of the standard. This paper describes an implementation of PTP with emphasis on the specification, design and performance of these algorithms. Included are initial acquisition of a master clock, tracking of the master clock, and rejection of outlying timestamps. Measured data from an operating system on switched Ethernet is presented.

- Application of IEEE 1588 to Distributed Motion Control: Kendal R. Harris, Sivaram Balasubramanian, and Anatoly Moldovansky, Rockwell Automation Abstract: This paper discusses the application of IEEE 1588 to distributed motion control systems. Current solutions rely on proprietary implementations to time synchronize distributed motion control system components. With the introduction of IEEE 1588 these solutions may now 
be implemented using open networks and standard components. Both peer to peer controller and controller to drive systems are considered.

- Proposal for IEEE1588 use over Metro Ethernet Layer 2 VPNs: Glenn Algie, Senior Advisor, Wireless Technology Labs, Nortel Networks Abstract: Metro edge and core deployed timing sources, receivers and inter-working devices can be a mix of Metro provider owned and managed vs. Enterprise owned and managed. A jitter tolerant recovery method is proposed where fewer Boundary clocks are required in the L1/L2 switched Metro. The presentation proposes a small set of enhancements to IEEE1588 to enable a suite of Metro Ethernet use cases.

Demonstrations, 2:30-3:30 PM:

- OnTime Networks AS, Øyvind Holmeide, Managing Director Description: Industrial managed Ethernet switch with Boundary clock functionality. 8 10/100BASE ports with any combination of FX and TX type. SNMP v2c, IGMP snooping, layer 2 and layer 3 QoS. The Boundary clock implementation supports: HW time stamping of IEEE-1588 or SNTP/NTP time packets, 1 PPS output signal. No IEEE-1588 management support

- Oregano Systems, Nikoaus E. Keroe

Description: An complete evaluation system will be presented consisting of four nodes together with a standard switch which is enhanced by a special time stamping unit able to perform both SynUTC high precision time stamping and boundary clock functions. Each computing node will offer a $1 \mathrm{pp}$ pulse and several other phase locked clock signals together with sensor inputs and actuator outputs. We will demonstrate the accuracy with a simple application. The basic IEEE1588 functionality and compatibility will be shown together with the extended features of the SynUTC approach (fault tolerance, ensemble clock and the like).

- Hirschmann Electronics, Dirk Mohl \& Dominik Iadonisi Description: Modular Ethernet switch with full IEEE 1588 implementation (hardware time stamping, best master clock algorithm, boundary clock and management: PTP/SNMP). Two switches will synchronize each other with an accuracy of about 100ns and synchronize a PC running Windows $2 \mathrm{k}$ and a legacy software implementation of IEEE 1588 with an accuracy of about $100 \mu \mathrm{s}$.

- Rockwell Automation, Kendal R. Harris and Sivaram Balasubramanian Description: Ethernet bridge with non-1588 boundary clock. Used for synchronizing remote chassis and distributed motion control. Network: 10/100BaseT. Will implement all of the functionality defined in 1588 except for best master clock algorithm \& associated functionality. This is a hardware-assisted implementation with synchronization accuracies in 50-500 nanoseconds range.

- GE Drives \& Controls, Inc., Salem, VA, Mark Shepard Description: An equipment demonstration will be presented using components from the GE SpeedTronic Mark-VIe Turbine Control System. A system controller, including a threeport boundary clock, and a thermocouple input node will be linked through a 100BaseT industrial Ethernet switched network. Both controller and node implement IEEE Std. 1588-2002 with hardware-assisted timestamps. Instrumentation to monitor the precision of time synchronization will be included. 
- Agilent Laboratories, John C Eidson \& Bruce Hamilton

Description: Prototype implementations of an earlier version of IEEE-1588 to demonstrate the effects of local oscillator stability on synchronization accuracy. Hardware assist and 10BaseT network technology is used. Internal visibility is provided by a web interface. 


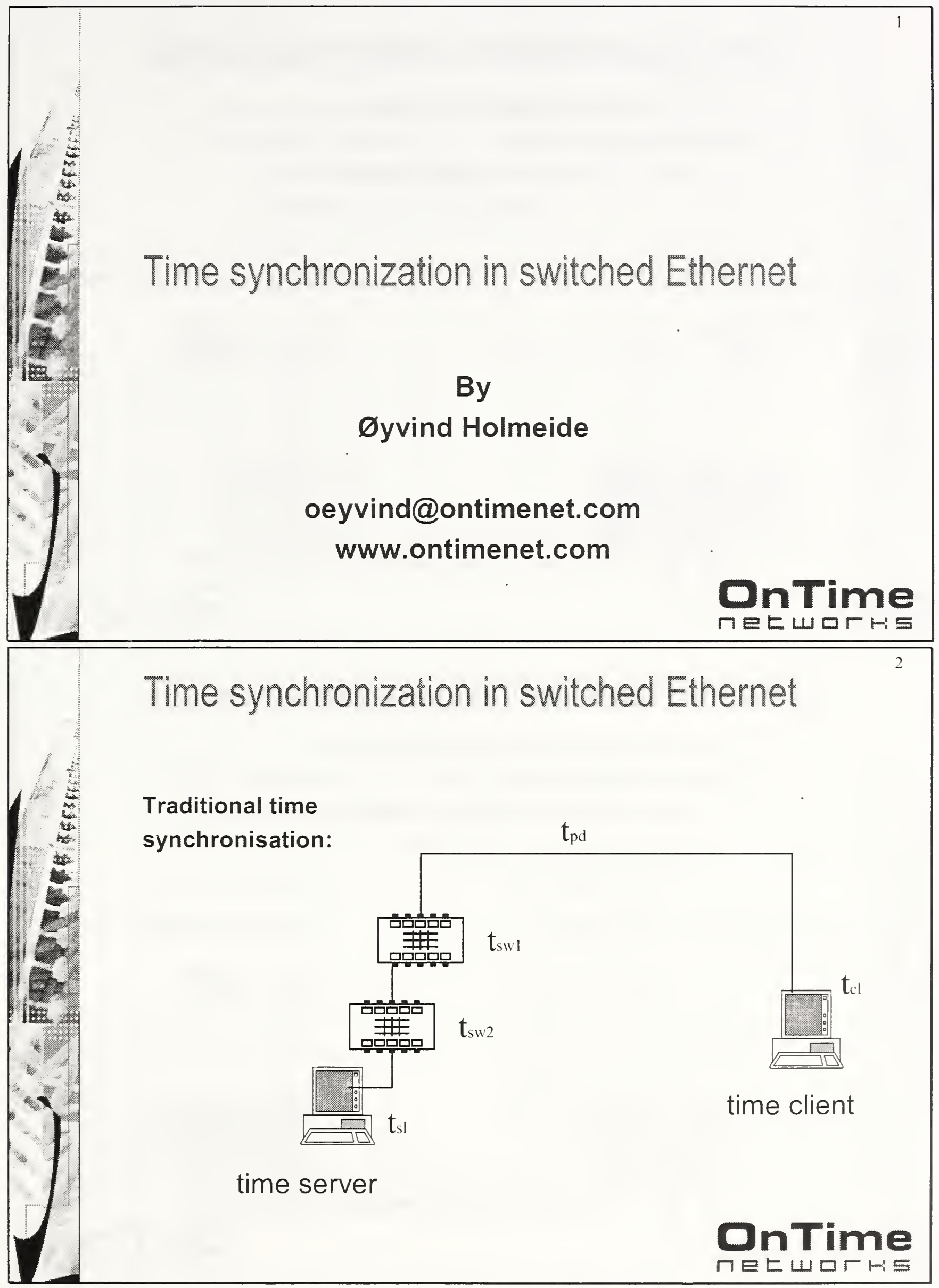




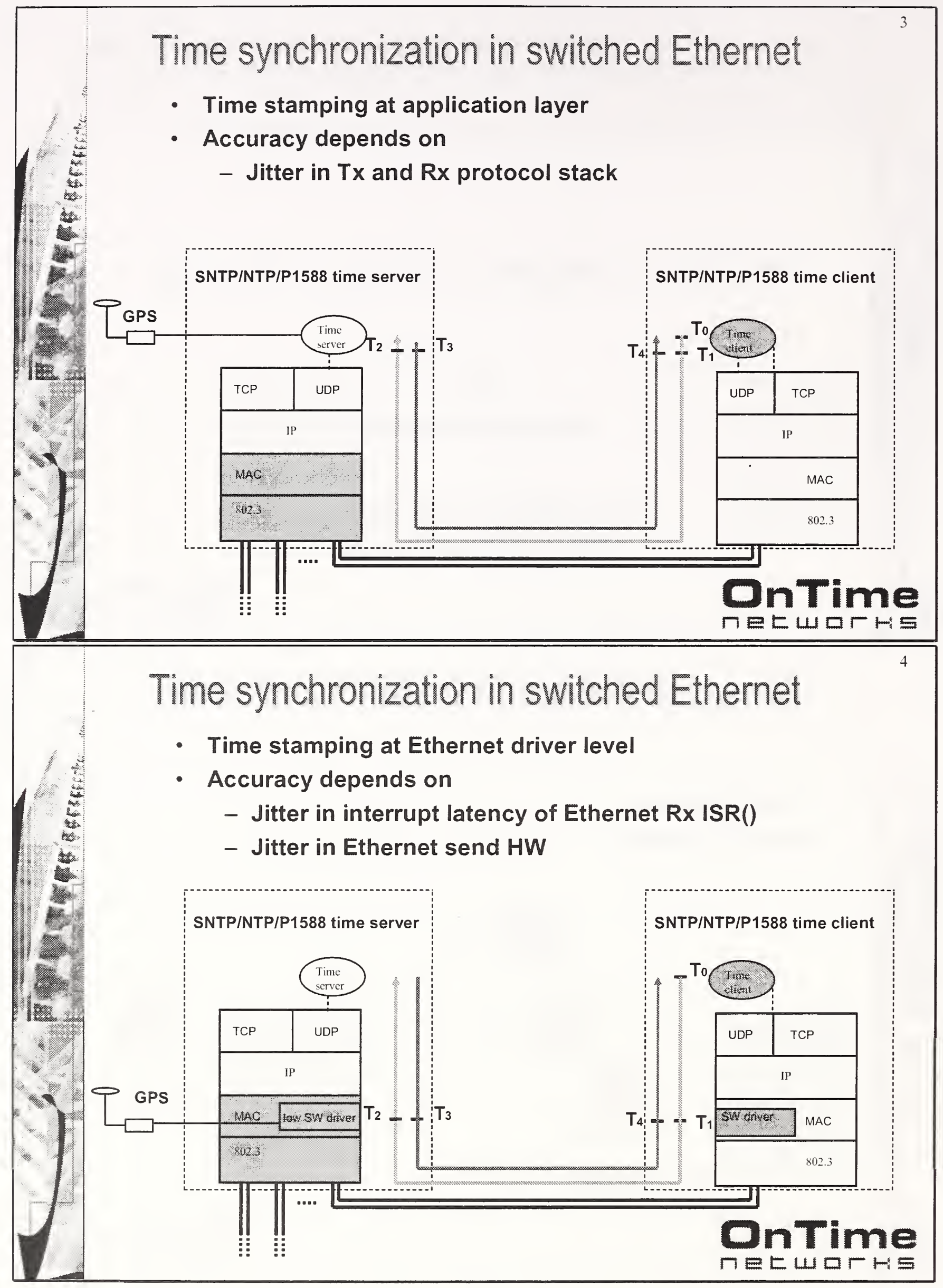




\section{Time synchronization in switched Ethernet}

- Time stamping at Ethernet HW

- Accuracy depends on

- GPS PPS rise time

- Stability of local oscillator

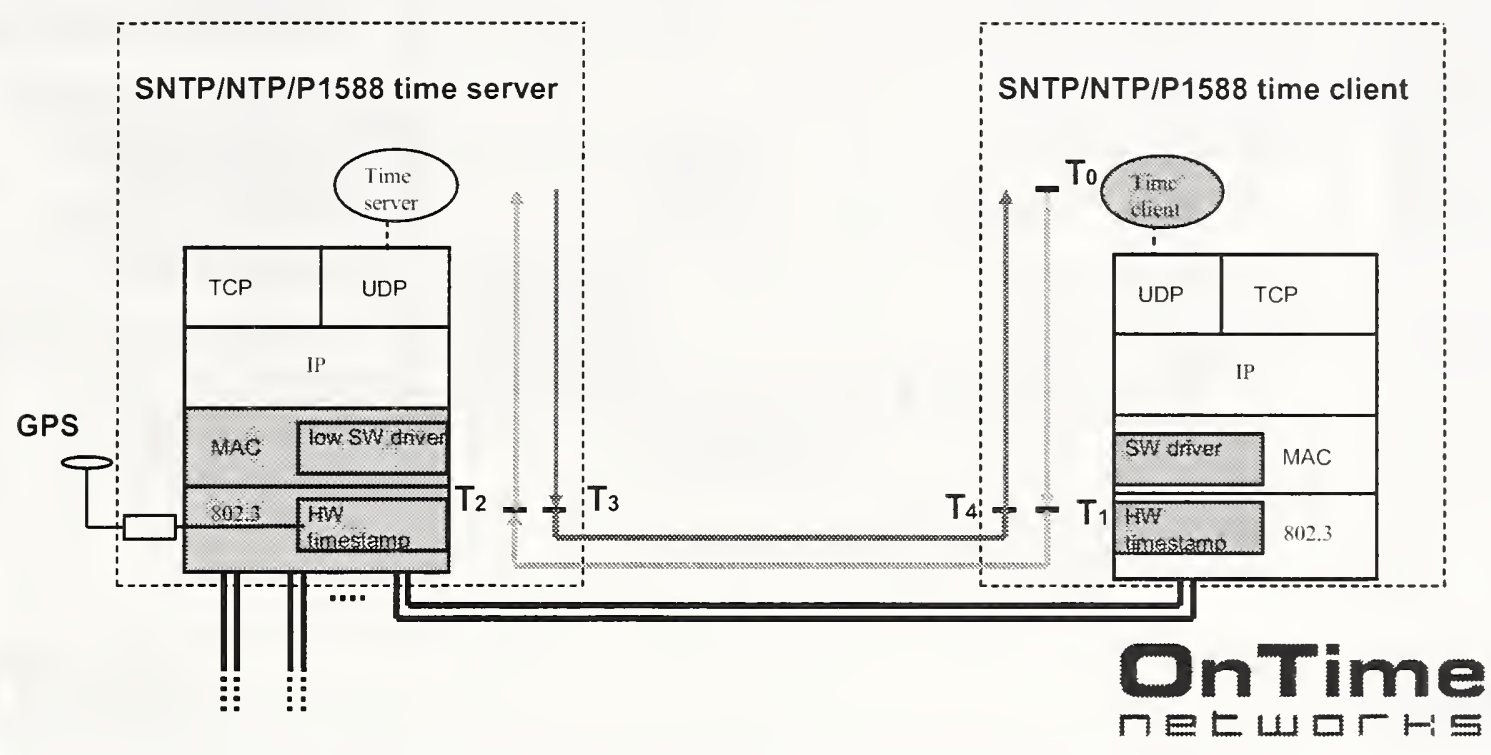

Time synchronization in switched Ethernet

How to achieve an accurate T3 time stamp in case

SNTP/NTP is used as the time server protocol:

- T3 is a future time that is generated by the switch CPU

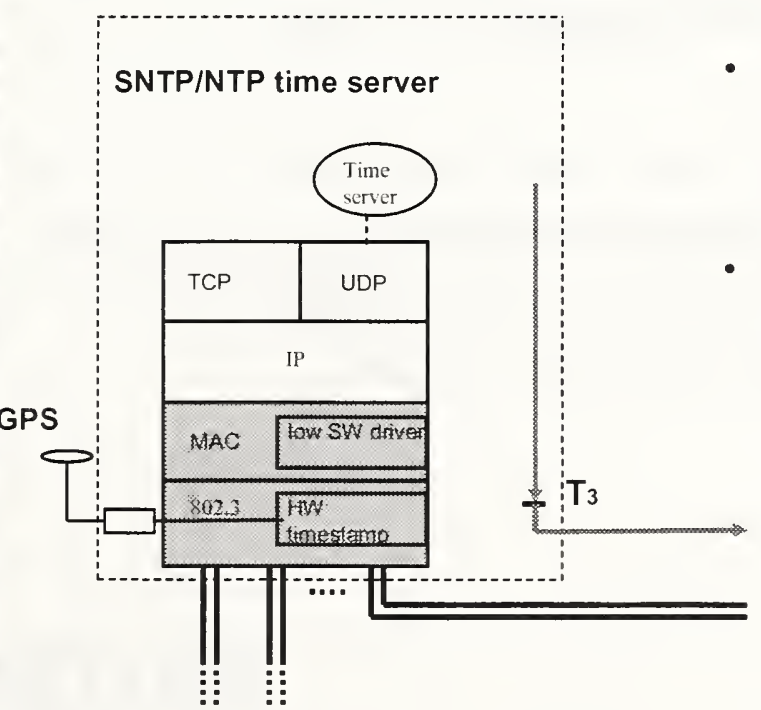

- The SNTP/NTP reply packet is sent from the switch when actual time is equal to T3 which is found in the SNTP/NTP payload

- Deterministic transmission of the SNTP/NTP reply packet can be achieved by using:

- Flow control (FDX - IEEE 802.3x)

- Back pressure (HDX)

- Transmission of a dummy packet 


\section{Time synchronization in switched Ethernet}

Delay through the Ethernet switch infrastructure depends on:

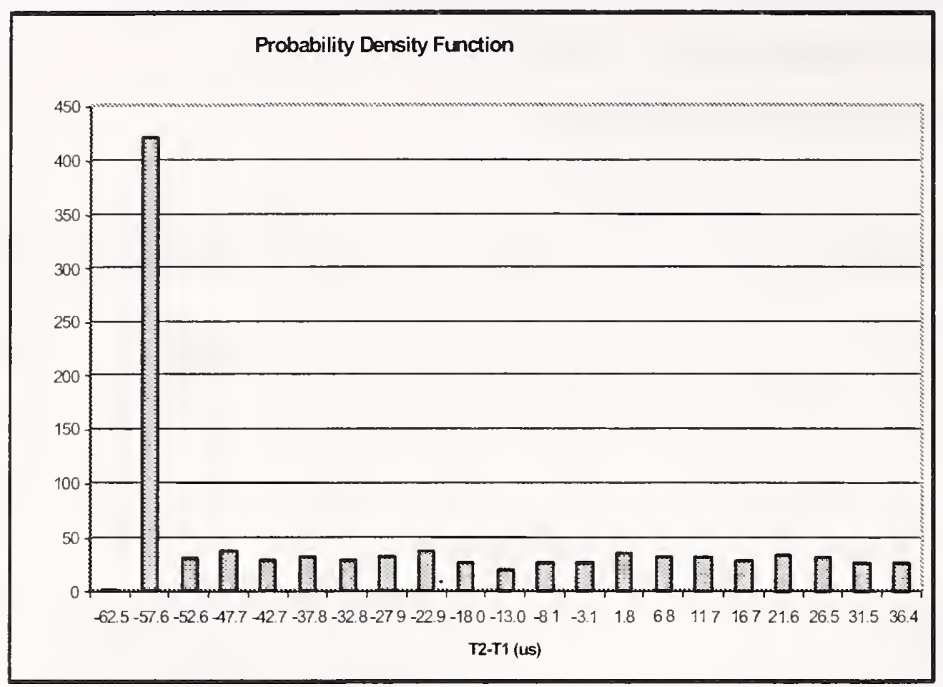

- Store-and-forward

- Network load

- Packet size

- Flow control

- Drop link speed

- Priority queues

Example on switch latency

OnTime metwark

\section{Time synchronization in switched Ethernet}

Filtering based on:

- NTP filtering techniques

- Erasures

- Detect and discard if time packets are extra delayed through switch queuing 


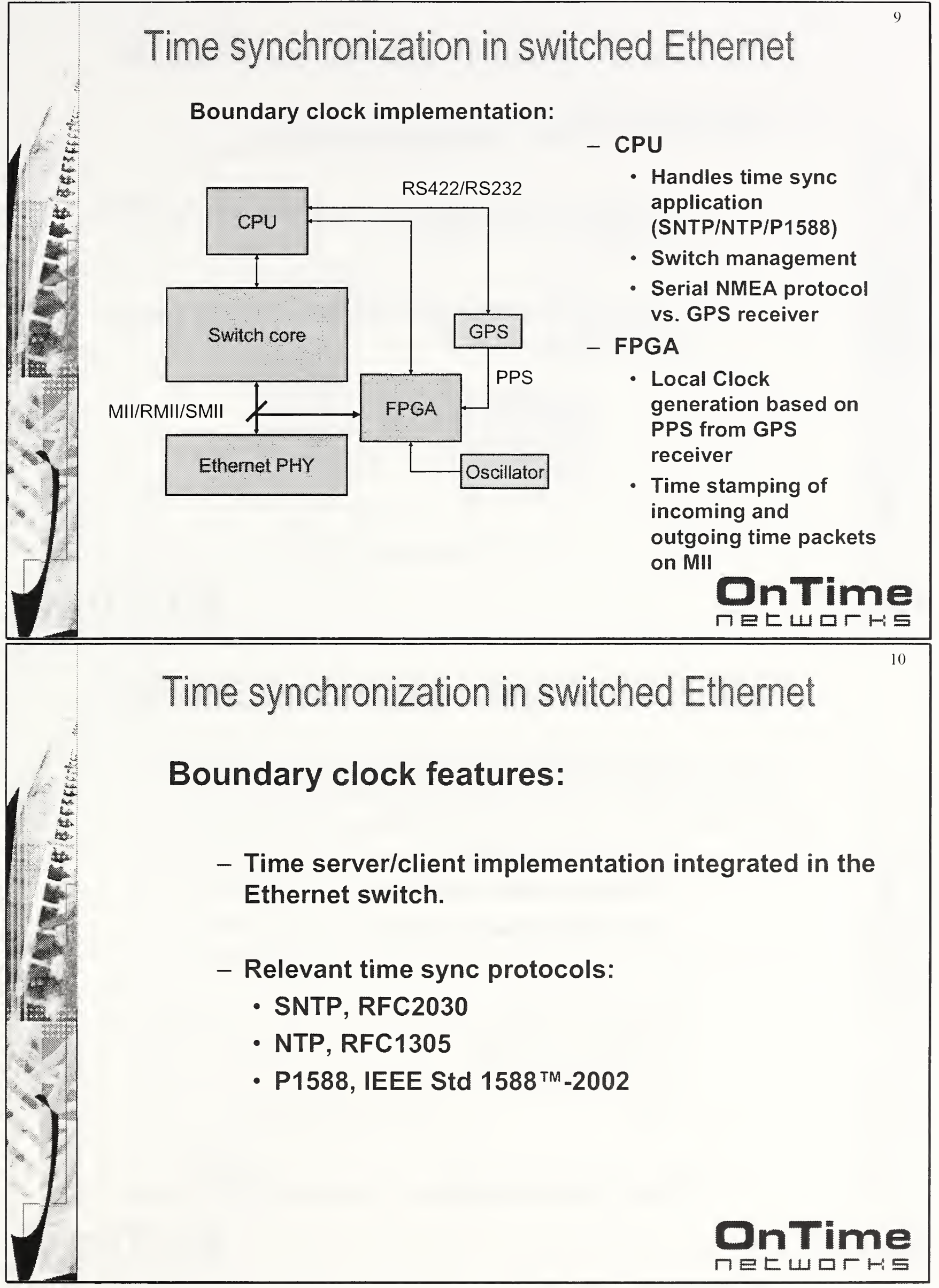


Time synchronization in switched Ethernet

\section{Boundary clock features cont'd:}

- Accuracy independent of network load, 0,1 PPM possible

- Forwarding of time sync packets only to switch CPU (P1588)

Time synchronization in switched Ethernet

Boundary clock features cont'd:

- Individual port state

- P1588: master, slave, etc

- NTP/SNTP: server/client

- Time reference:

- External GPS or

- Local clock

- Etc.

- Time synchronization protocol analyzer support 


\section{Time synchronization in switched Ethernet}

Boundary clock implementation with time synchronization protocol analyzer support:

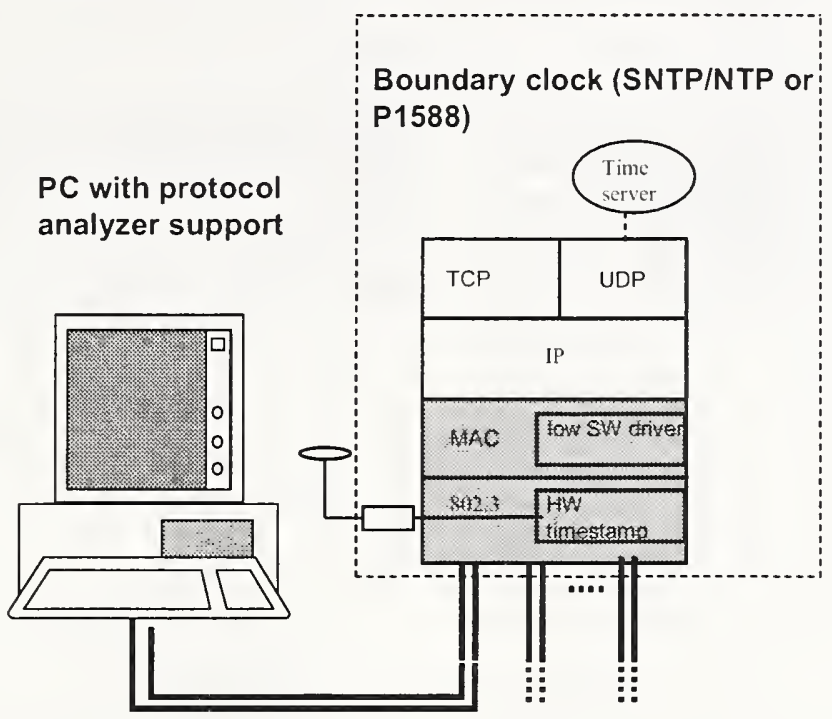

\section{Time synchronization in switched Ethernet}

- Boundary clock with protocol analyzer support

- All time sync packets (SNTP/NTP or P1588) sent and received on any port are sent to the $\mathrm{PC}$ for presentation

- PC with protocol analyzer support

- Directly connected to the Boundary clock

- Time sync packets are visualized

- Detailed presentation of the time sync packet content

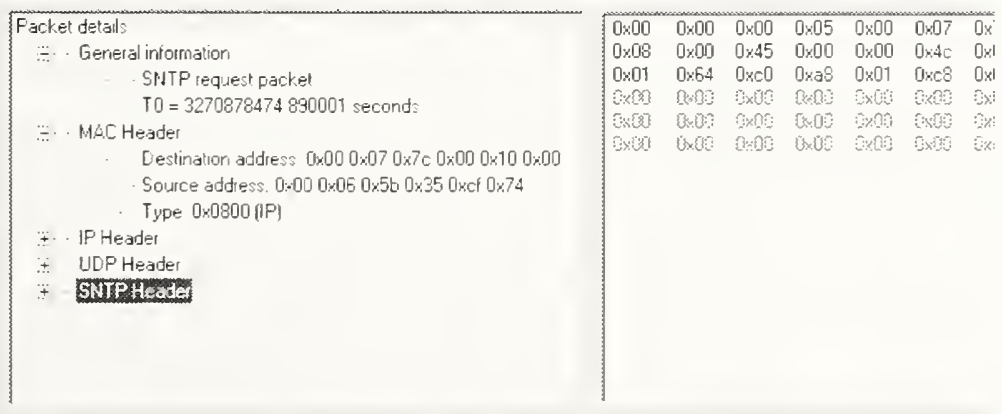




\title{
Boundary Clock Implementation with Time Synchronization Protocol Analyzer
}

\author{
Kristin Holmeide and Øyvind Holmeide
}

\begin{abstract}
Variable latencies through the protocol stacks and the Ethernet switches will degrade the timing accuracy that can be achieved when time synchronization is performed via a switched Ethernet infrastructure. Time stamping of incoming and outgoing time packets shall preferably be done as low as possible in the protocol stacks. The Ethernet switch latency depends on the network load and the switch architecture. This problem can be solved if time synchronization properties are integrated in the Ethernet switches as well as in the Ethernet end nodes. This article describes the basic principles of a P1588 Boundary Clock and the well established internet SNTP/NTP protocol and how to implement these protocols on Ethernet switches in order to achieve a timing accuracy in the sub-microsecond $(\mu s)$ range regardless of the network load. How a Boundary Clock implementation can be used as a time synchronization protocol analyzer is also described.
\end{abstract}

Index Terms-Time synchronization, switched Ethernet, SNTP/NTP, P1588, Time synchronization protocol analyzer.

\section{TIME STAMPING OF TIME PACKETS}

Timing accuracy depends on where the time stamping of incoming and outgoing time packets is performed. Time stamping can be performed at the application layer, Ethernet driver level (software) or at the Ethernet data link/physical layer (hardware).

\section{A. Time stamping at application layer}

Most time synchronization implementations perform all time stamping at the application layer, see Figure 1 for details. This means that the timing accuracy that can be achieved at the clients will suffer from the variable latency through the UDP/IP protocol stack. This latency depends on the UDP/IP implementation, platform load and the Real Time Operating System (RTOS) implementation. The impact of this jitter can be reduced by using the NTP or similar filtering techniques.
The timing accuracy that can be achieved at the time clients by performing time stamping at application layer will typically be in milliseconds range.

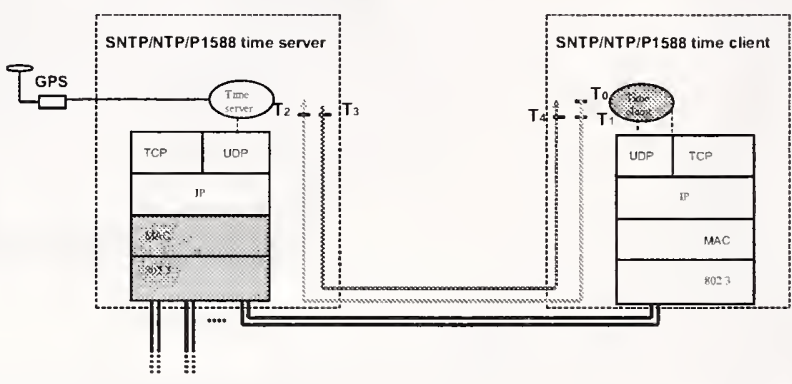

Figure 1, time stamping at application layer

The server adds both the receive time stamp of the time request, $T_{2}$, and the transmit time stamp of the time reply, $T_{3}$, in the time reply packets. The $T_{3}$ timestamp is put into the packet before the packet is sent.

\section{B. Time stamping at the Ethernet driver}

The timing accuracy can be significantly improved if the time stamping is performed at the Ethernet software driver level. The receive time stamps, i.e. $\mathrm{T}_{2}$ and $\mathrm{T}_{4}$, are performed in the Ethernet Interrupt Service Routine (ISR), while the transmit time stamps of the client, $\mathrm{T}_{1}$, is performed in the Ethernet send routine of the Ethernet driver. The $T_{3}$ time stamp is somewhat more tricky, since this time stamp is already included in the reply packet that is generated in the application layer. How to achieve an accurate $T_{3}$ time stamp is further discussed in chapter II. This is relevant in case SNTP or NTP are used as time synchronization protocols. 
It is possible to store the server clock when the time reply was sent from the server, $\mathrm{T}_{3}$, and send this time stamp in a second packet later. This is one of the main properties of the P1588 protocol. The time reply is referred to as a SYNC packet and the second packet is referred to as the FOLLOW_UP packet.

Transmit time stamping can be very accurate; while the corresponding receive time stamps may suffer from jitter in the interrupt latency. This jitter depends on the RTOS implementation and the platform load. An alternative software approach that can be used in order to overcome this problem is to implement an RTOS independent ISR that performs the time stamping of incoming time packets.

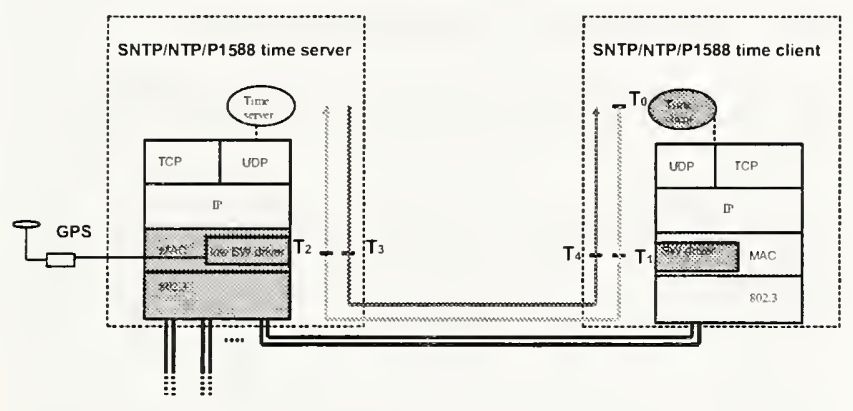

Figure 2, time stamping in Ethernet level

\section{Time stamping at the Ethernet data link/physical layer}

The latency through the protocol stacks can be removed if time stamping is performed in the data link/physical layer. The time stamping can be implemented in HW in the Ethernet controller, the PHY chip or in e.g. a separate FPGA that is interfacing the Media Independent Interface (MII) between the Ethernet controller and the Ethernet PHY chip. Time synchronization can be extremely accurate if time stamping is performed in HW. The accuracy at the time clients can be better than 1 PPM if this method is used and a direct wire is used between the server and the client. (Achieving an accurate $\mathrm{T}_{3}$ time stamp is also a challenge when using this method, see chapter II for how this can be done.)

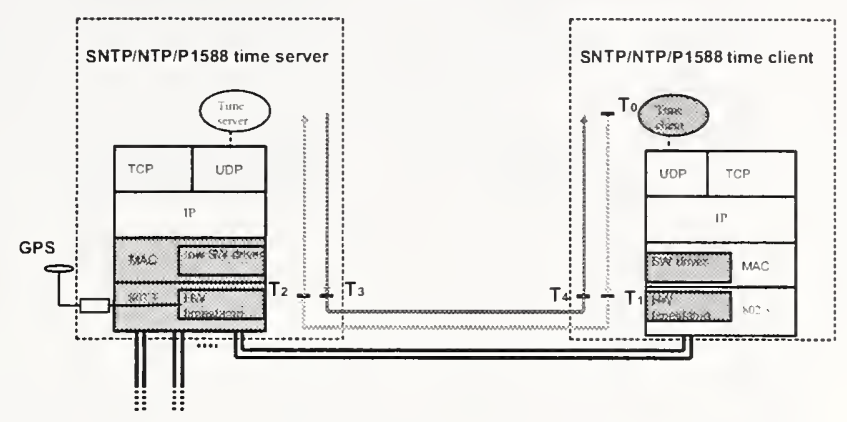

Figure 3, time stamping at Ethernet data link/physical layer

\section{HOW TO ACHIEVE AN ACCURATE T3 TIME STAMP ${ }^{1}$}

The SNTP time reply contains both the receive time stamp of the time request packet, $\mathrm{T}_{2}$, and the transmit time stamp, $\mathrm{T}_{3}$, of the time reply packet. Thus, actual time must be equal to $T_{3}$, when the time reply packet hit the drop link. This means that the server must have deterministic access to the media. I.e. $T_{3}$ is a future time when this time stamp is put into the time reply, and the packet will then be sent on a pre-determined point in time. The following implementations can be used:

1. The flow control feature of the Ethernet switch, ref. IEEE802.3x, can be used in case of full duplex connectivity in order to hold back the reply packet until absolute time is equal to the $\mathrm{T}_{3}$ time stamp given in the time reply payload. The time reply packet must be ready for transmission when the flow control is turned off.

2. The back pressure feature of the Ethernet switch can be used in case of half duplex connectivity in order to hold back the reply packet until absolute time is equal to the $T_{3}$ time stamp given in the time reply payload. The time reply packet must be 
ready for transmission when the back pressure signal (JAM patter) is turned off

3. Sending a dummy packet of a given length can be used for both half and full duplex connectivity drop links. The time reply packet must be sent immediately after the dummy packet (only minimum Inter Packet Gap (IPG) between the packets). The time reply packet is granted access to the media at a given time, T3, by using this technique.

Note: the time reply packet may experience a collision in case of half duplex connectivity, and a re-transmission of this packet will then contain a wrong $\mathrm{T}_{3}$ time stamp. This must be handled either at the server (i.e. abort retransmissions of time reply packets) or at the client (the client generates an erasure in case a collision is detected prior to the reception of the time reply packet).

\section{WHY IS SWITCH LATENCY A PROBLEM?}

Figure 4 shows a traditional time synchronization implementation, where time packets are sent through a switched Ethernet infrastructure.

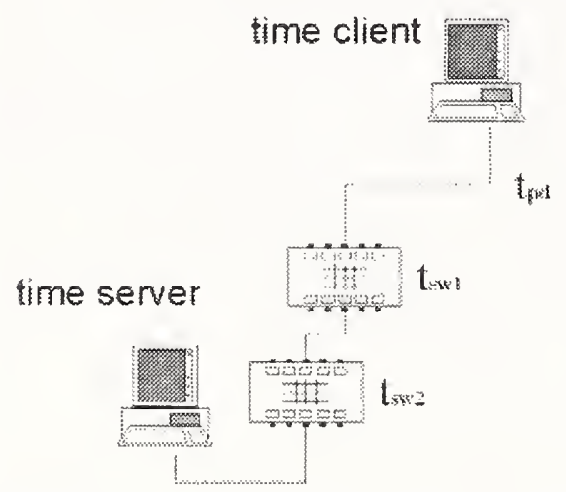

Figure 4, traditional time synchronization

The network latency depends on the network load, drop link speed, packets sizes, the switch architecture and the number of switches between the server and the client. The switch latency may vary from a few tens of microseconds up to several milliseconds.

Most new Ethernet switch designs are based on "store-and-forward" technology. This means that an Ethernet packet must be completely received on an input port before it is checked for bit errors (the switch calculates the Frame Check Sequence (FCS), and compares it with the FCS found at the end of the packet) and forwarded to the respective output port. Thus, the latency depends on the drop link speed and the packet sizes. E.g. an Ethernet packet of maximum Ethernet packet size (1522 bytes) received on a $10 \mathrm{Mbps}$ drop link will be delayed by 1.2 milliseconds due to the "store-and-forward" mechanism. A corresponding 100 Mbps drop link represents a delay of 122 microseconds.

The packet may be further delayed if other packets are queued for transmission on the same output port. Protecting the time packets by using priority does not improve the situation, because the transmission of another packet may already be started when the high priority time packet enters the output port queue. Figure 5 shows an example of the Probability Density Function (PDF) of the switch latency taken from actual measurements in case other packets are sent to the same output port as the time packets. The measurement is based on $50 \%$ load on the receiving drop link. The PDF has a uniform distribution for all switch latency measurements where extra delay is introduced due to other packets.

Note: extra latency due to other traffic is also a problem in case the switch is based on "cutthrough" packet forwarding.

The switch latency may also vary due to general switch load. I.e. packets sent and received on ports not used for time updates. Such an unwanted switch property is only relevant on old switch architectures. 

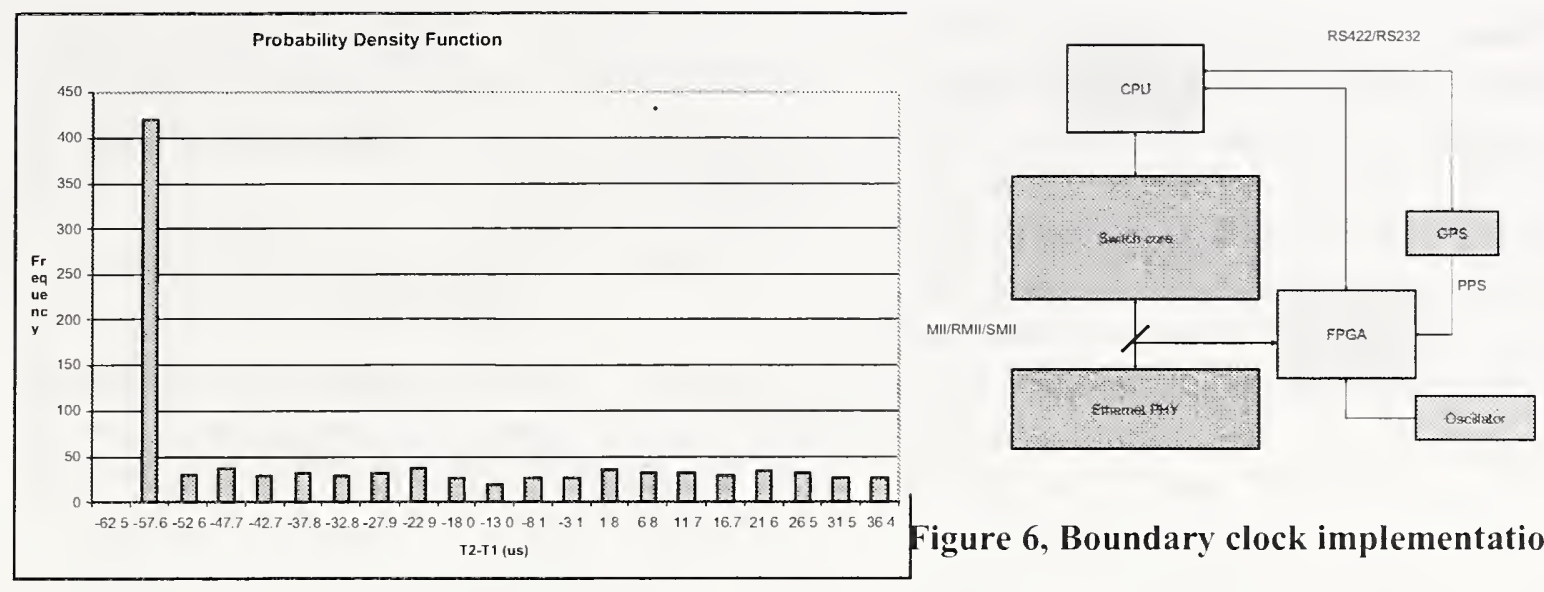

Figure 6, Boundary clock implementation

Figure 5, example of switch latency measurements

The NTP filtering techniques can be used to reduce the impact of variable network latency. However, these filtering techniques are not optimized for typical latency density functions relevant on a LAN based on Ethernet switches. A better accuracy can be achieved if knowledge about general switch latency characteristics is utilized. Time updates performed in a LAN network, where there is one Ethernet switch on the path between the time client and the time server, will suffer from variable switch latency caused by the switch network load as earlier described. However, both the time client and the time server can verify if a received time packet is extra delayed through the switch, by another packet being sent from the switch. The extra delay is verified if the interval between this preceding packet and the time packet, is equal to the minimum Inter Packet Gap of Ethernet or close to this interval ${ }^{2}$. A convenient implementation of this erasure technique, in case the time synchronization implementation is based on a software time stamping of incoming time packets in the Ethernet ISR, is to generate this type of erasure if the time packet received is not the first received packet associated to the Ethernet receive interrupt.

\section{BOUNDARY CLOCK IMPLEMENTATION}

A Boundary clock implementation is shown in Figure 6.

\footnotetext{
${ }^{2} \mathrm{ABB}$ patent pending
}

Incoming and outgoing time packets are time stamped in hardware at the Media Independent Interface (MII) between the switch core and the Ethernet PHY. Thus, an incoming time packet is time stamped before it is forwarded through the Ethernet switch core and an outgoing time packet is time stamped after the packet has been sent through the switch core. This means that variable latency through the switch core has no impact on the time synchronization accuracy. The time stamping is performed in an FPGA. The FPGA also generates the local clock of the Boundary clock implementation based on either an external Pulse Per Second (PPS) input from e.g. a GPS receiver, or only based on a local oscillator (e.g. the switch core oscillator). The drift and offset of the local oscillator is adjusted based on the PPS signal in case an external time base is used.

The CPU handles the time sync protocol, Boundary clock configuration via e.g. SNMP, serial interface versus an external clock source (if available) and the interface versus the FPGA. The NMEA protocol over RS232 or RS422 versus an external GPS is often relevant in order to have reference to absolute time. RS422 is the preferred interface for both serial data and the PPS signal in order to meet various installation requirements (distance between GPS receiver and Boundary clock). The most relevant time synchronization protocols are based on SNTP/NTP (RFC2030/RFC1305) or P1588 (IEEE Std $\left.1588^{\mathrm{TM}^{\mathrm{M}}}-2002\right)$. These protocols are all based on UDP/IP.

An Ethernet network where all network elements are based on Boundary clock 
implementation should not forward time synchronization packets from one Ethernet port to another Ethernet port. Time synchronization packets received on a Boundary clock port should be forwarded to the Boundary clock CPU only in order to make sure that there is no network element on the path between the time client and the time server. This property is included in the P1588 standard, but not in the SNTP or NTP standards. However, a SNTP/NTP client might be able to verify the number of network hops (switches) between the client and a given server based on the stratum field of the SNTP/NTP reply from a SNTP/NTP server or based on the propagation delay between the client and the server. The stratum field and/or propagation delay measurement performed by the client are used in order to choose the best SNTP/NTP server alternative. I.e. preferably a SNTP/NTP Boundary clock implementation directly connected to the SNTP/NTP client. A network hop can be identified based on the store-and-forward delay introduced by the network element. Such a SNTP/NTP property ought to be implemented on a SNTP/NTP Boundary clock.

The ports of a Boundary clock may have different states. E.g. one port may act as a time client versus a better time server, while all the other ports act as time servers. This is a fundamental principle of the P1588 standard. In P1588 context this means that the time client port is in SLAVE state and the other ports on the Boundary clock are in MASTER state.

The Boundary clock accuracy in case an external time base is used, depends on the quality of the PPS signal from an external clock source (rise time of PPS and jitter between the PPS signals) and the jitter per second of the local oscillator. The latter degradation factor is often depending on temperature variation unless the local oscillator has a temperature compensation property. A quality parameter can be calculated based on the jitter per second of the local oscillator. This parameter can be calculated for a given time interval and associated to time updates performed within this time interval. This parameter is referred to as the clock variance in the P1588 standard. An accuracy in the order of 0,1 PPM is possible.

\section{TIME SYNCHRONZATION PROTOCOL ANALYZER}

A Boundary Clock implementation can also function as a time synchronization protocol analyzer together with a PC directly connected to Boundary Clock. The PC is running a program for configuration and visualization of time synchronization packets sent and received on any of the ports on the Boundary Clock. Both the P1588 and the SNTP/NTP protocols can be supported. Such a program for network capture and a Boundary clock with the capability of trapping and sending time synchronization packets to the connected PC, can be an excellent tool during the development of time synchronization support based on P1588 or SNTP/NTP of an Ethernet enabled end node.

The multicast client part of the network capture program connects to the corresponding multicast server of the Boundary Clock implementation via a predefined IP multicast socket address and port number. Multicast packets sent from the multicast client to the Boundary Clock are only forwarded to the Boundary Clock CPU. No knowledge about the Boundary Clock IP address is required since IP multicast communication is used between the PC and the Boundary Clock.

All P1588 or SNTP/NTP packets sent or received on any ports of the Boundary Clock including the port where the $\mathrm{PC}$ is running the network capture program, will be trapped on the Boundary Clock and sent to the PC via the established multicast socket.

The time synchronization packets are visualized per port in the same order as they appear in time on the Boundary Clock. The user can select any of the trapped time synchronization packets for a detailed packet view. A time synchronization packet is presented as shown in Figure 7. 


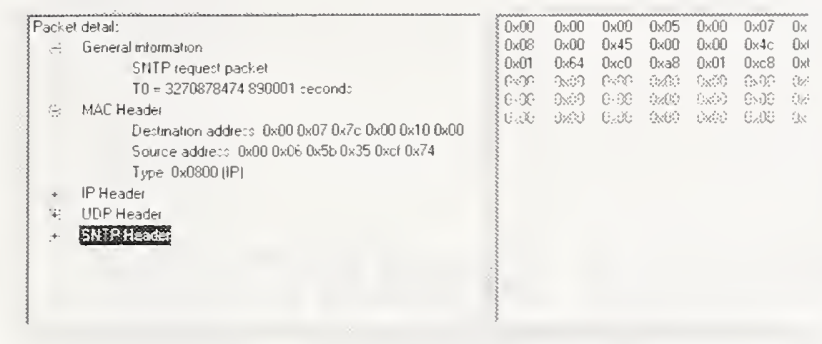

Figure 7, visualization of a time synchronization packet

The whole packet and each field can also be presented in hex format representation. Tool tip text is associated to relevant fields of the time synchronization packet payload. The time stamps relevant for exact time synchronization can easily be extracted for e.g. the purpose of correct calibration and/or proper time synchronization on a P1588 or SNTP/NTP platform under development.

A P1588 or SNTP/NTP implementation can be compared versus the standard or other similar time synchronization implementations by using this tool. Interoperability problems related to time synchronization by using the P1588 or SNTP/NTP protocols can also be easily identified.

Time synchronization communication on all ports on the Boundary Clock can be inspected from the same tool, without any other network elements (i.e. hubs) on the drop links between the Boundary Clock and the time synchronization end nodes. 


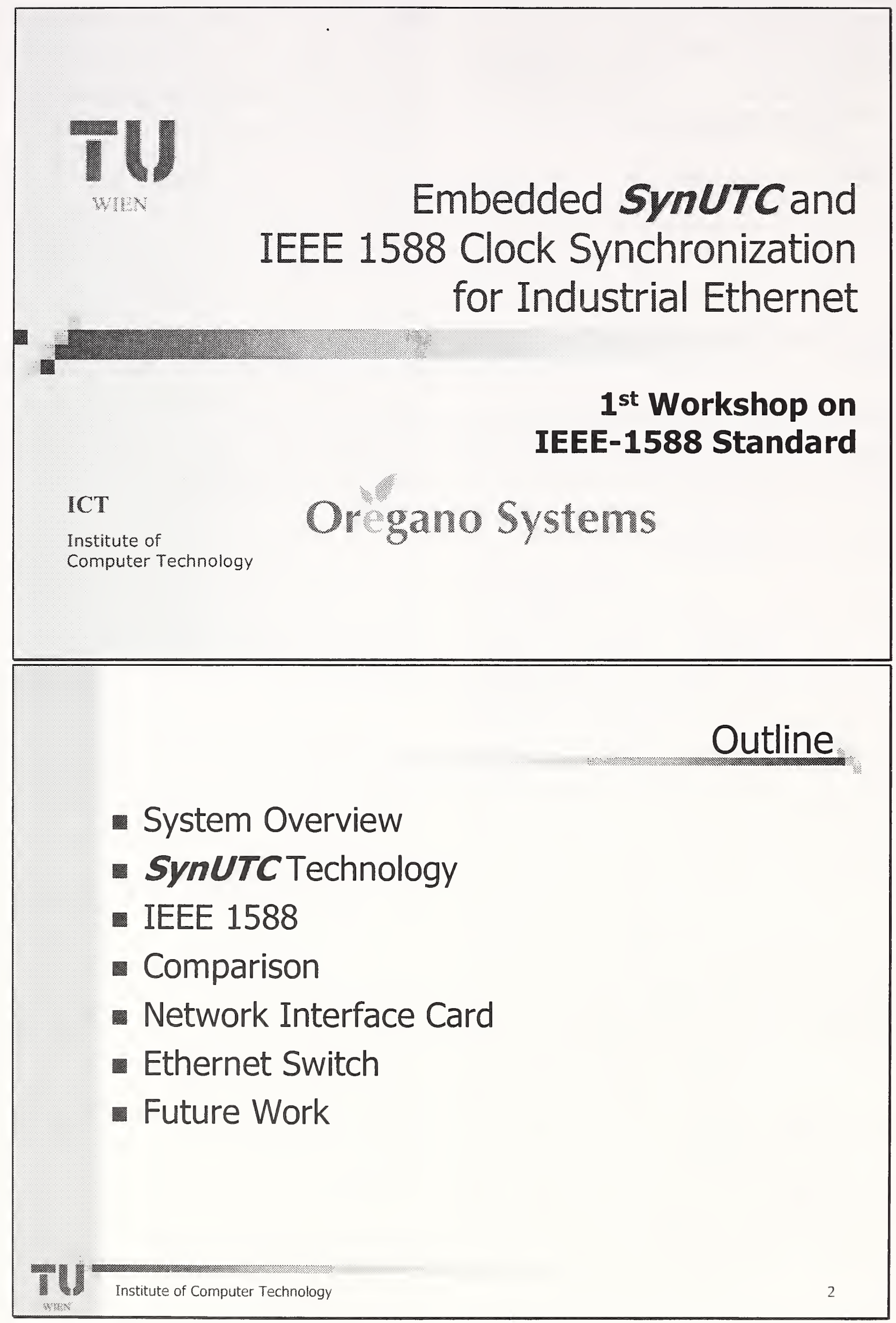




\section{System Overview}

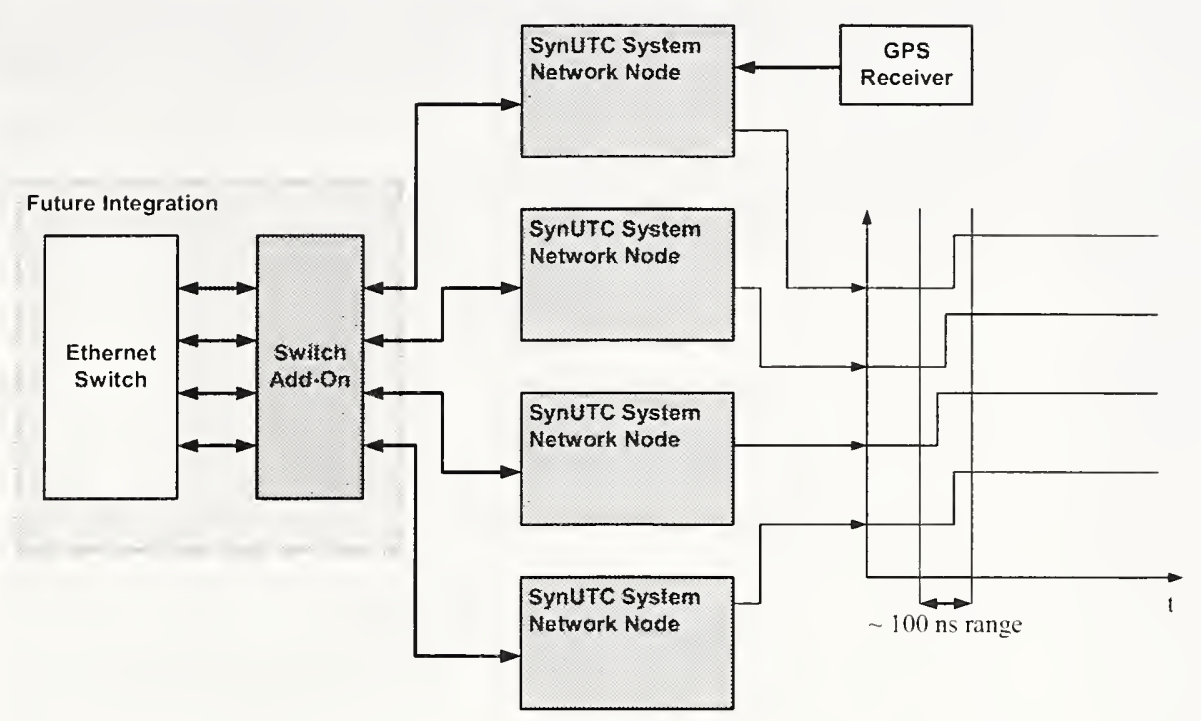

\section{SynUTCTechnology}

- Adder based clock

- On-the-fly timestamping

- Interval based paradigm (pos/neg accuracies)

- Clock state and rate synchronization

- Non master-slave principle

- Covers synchronization algorithms

- Covers hardware implementation issues

- Strong mathematical background 


\section{SynUTCAdder Based Clock 1}

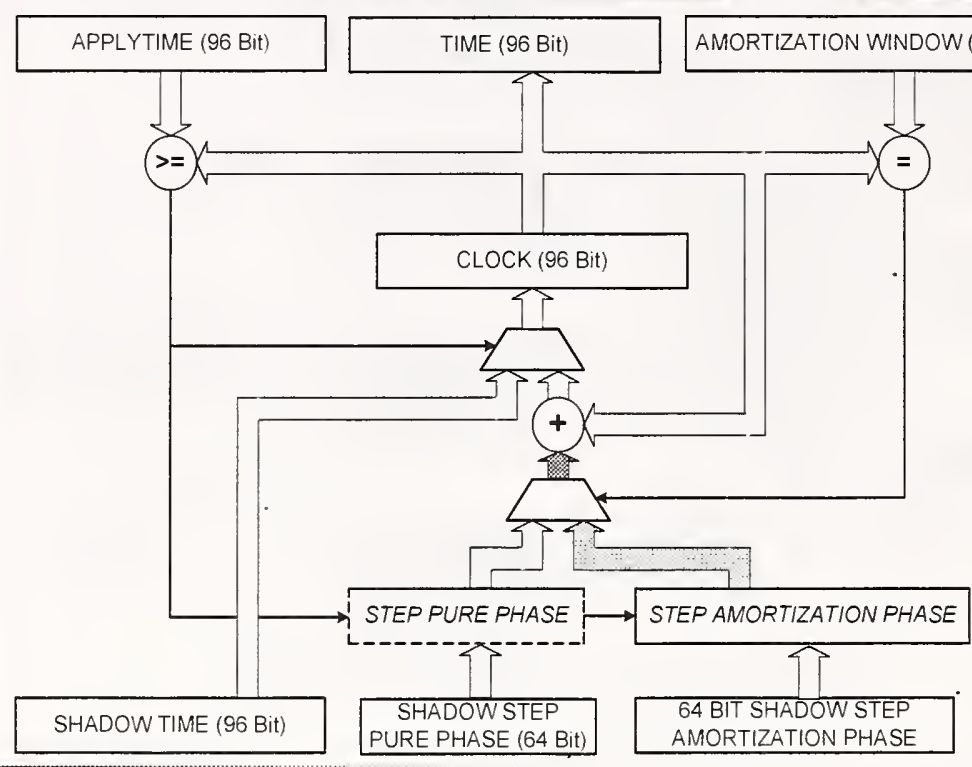

\section{SynUTCAdder Based Clock 2}

IEEE 1588 + PSynUTC Adder Based Clock Register Layout

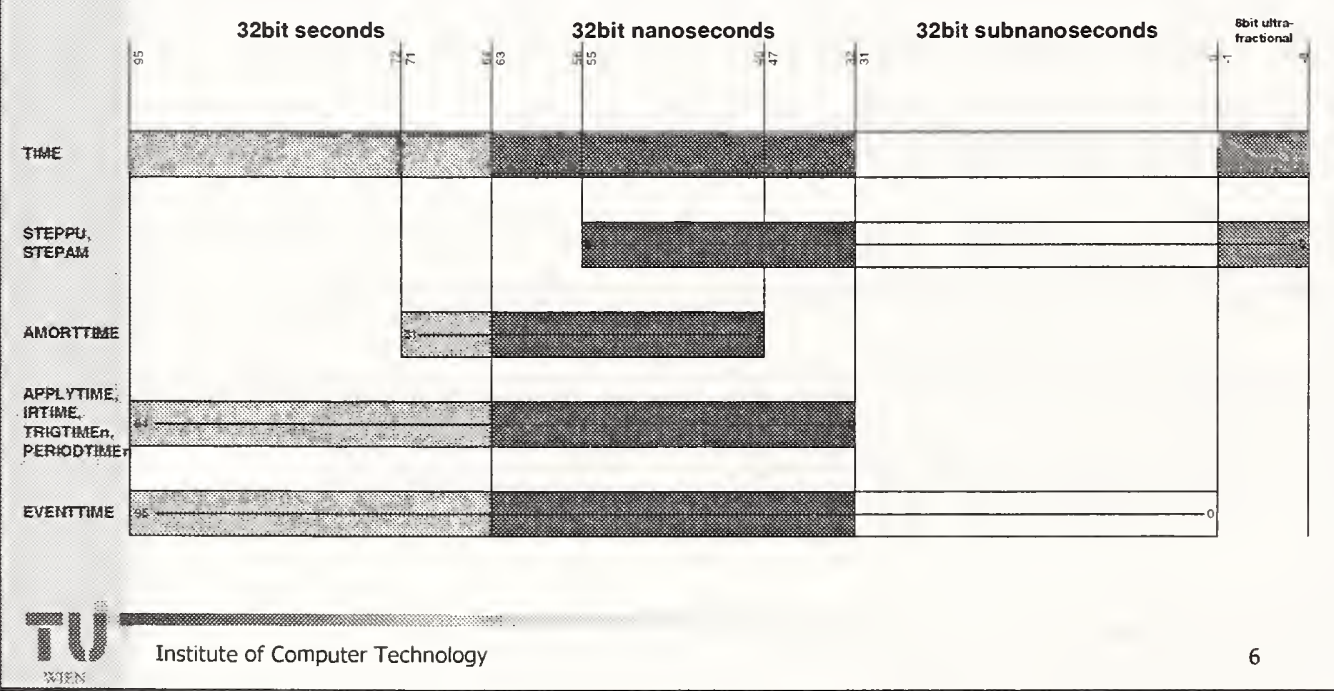




\section{SynUTC Adder Based Clock 3}

\begin{tabular}{|c|c|c|c|}
\hline $\begin{array}{l}\text { Number of } \\
\text { Pipeline } \\
\text { Slages }\end{array}$ & $\begin{array}{l}\text { FPAI (CMOS } \\
0.18 \mu \mathrm{m})[\mathrm{MH}]\end{array}$ & $\begin{array}{l}\text { FPA2 (CMOS } \\
0.18 \mu \mathrm{m})[\mathrm{MHz})\end{array}$ & $\begin{array}{l}\text { ASIC (CMOS } \\
0.35 \\
{[\mathrm{MH}]}\end{array}$ \\
\hline \hline 0 & 114 & 82 & 242 \\
\hline 1 & 139 & 128 & 257 \\
\hline 2 & 166 & 174 & 247 \\
\hline 5 & 155 & 181 & 293 \\
\hline 10 & 134 & 192 & 297 \\
\hline 20 & 122 & 210 & 383 \\
\hline 30 & 123 & 216 & 396 \\
\hline 47 & 110 & 245 & 443 \\
\hline
\end{tabular}

\section{SynUTC Adder Based Clock 4}

- Core for 2 MACs approx. 40k gates

(130 MHz @ $0.18 \mu \mathrm{m}$ CMOS)

- NIC FPGA design in Altera Stratix 1S25F672

(20k LEs for all cores @ 33/50 MHz)

- SAO FPGA design in Altera Stratix 1S25F672

(4k LEs @ $50 \mathrm{MHz}$ )

- Long adder can be easily pipelined

higher clock rates

Seamless FPGAs implementation 


\section{Intervals and Continuous Amortization}

Clock Time

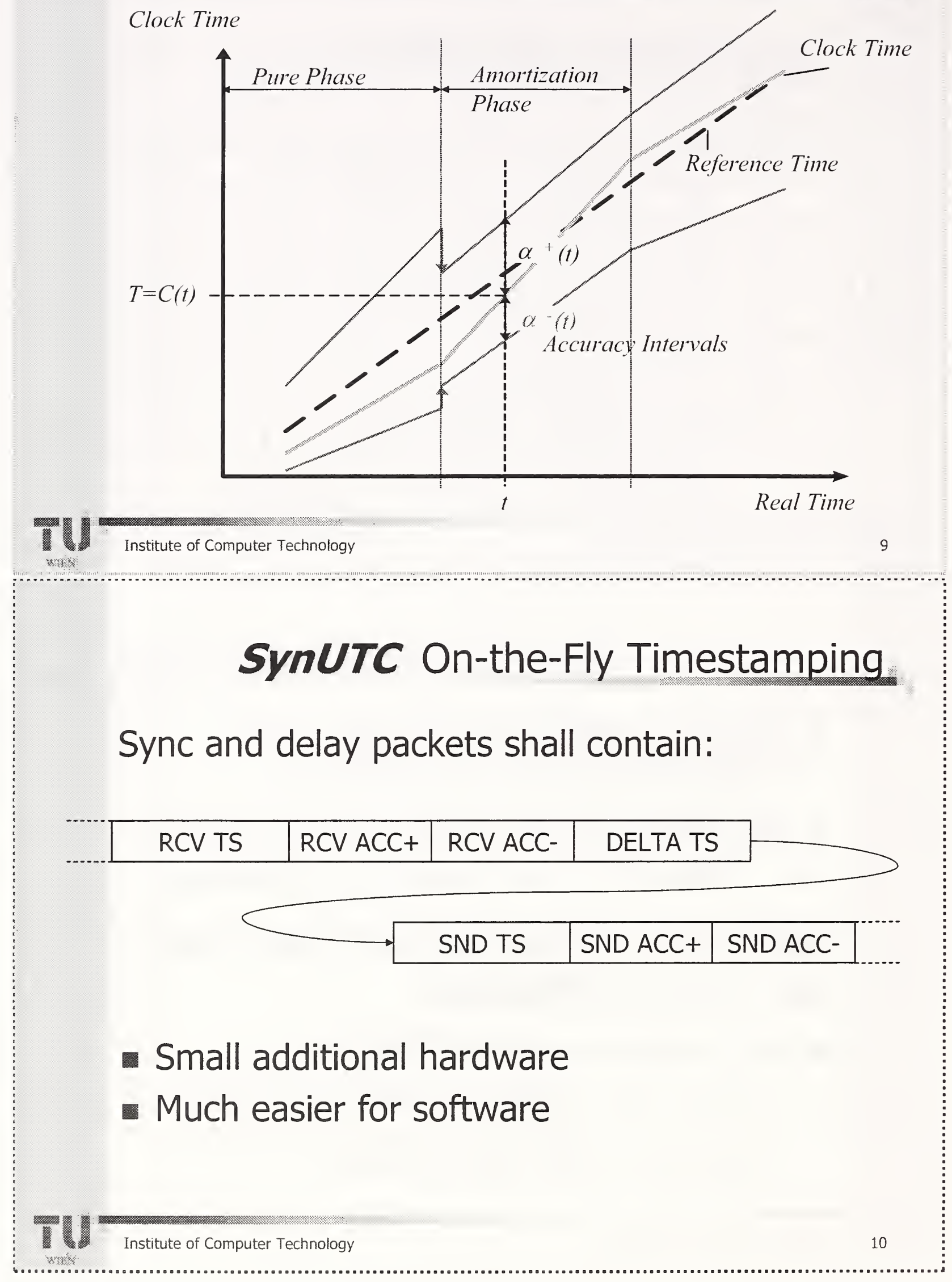




\section{IEEE 1588}

- Small networks

- Consuming small amount of resources

- Small administrative overhead

- Master-slave principle

- Automatic network partitioning

- PTP (Precision Time Protocol) clock definition

- BMC (Best Master Clock algorithm)

\section{SynUTC VS. IEEE 1588}

- Improved fault tolerance, due to its NON master-slave principle

- Clock accuracy information for all clocks (eases control loops, ...)

- On the fly packet timestamping

- Algorithms for state and rate synchronization of the clocks

- Support for external clock synchronization 

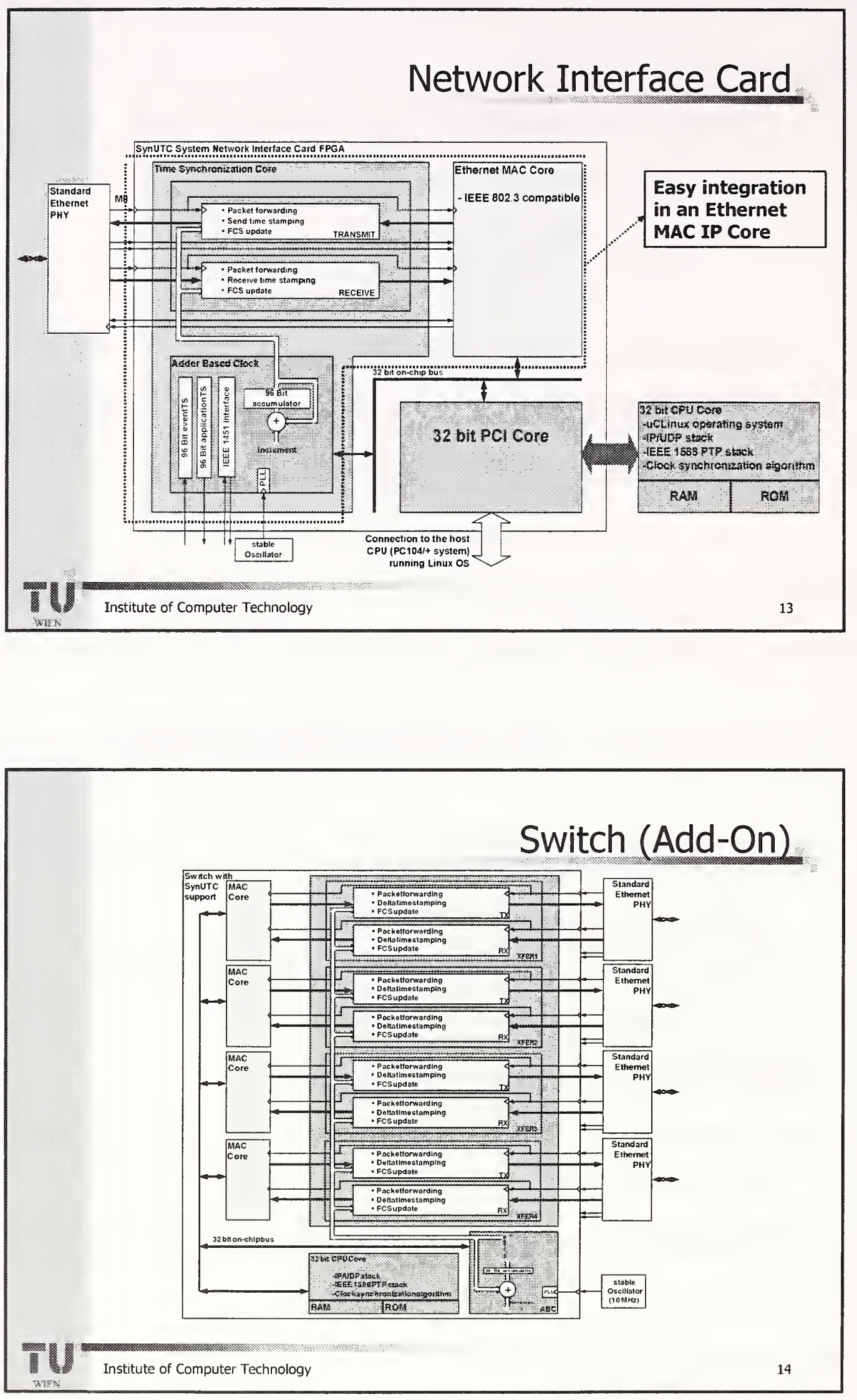


\section{Ethernet PHY Evaluation 1}

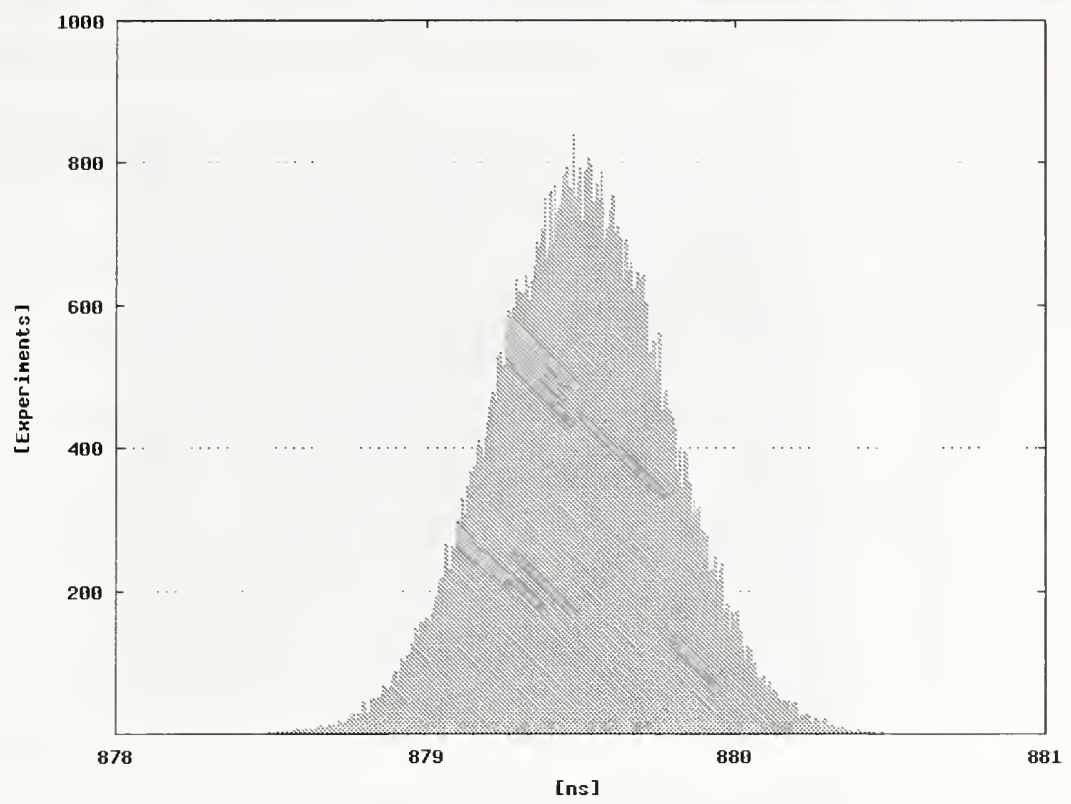

Jitter of 100.000 clock synchronization packets for

Fast Ethernet PHY's, 99m CAT-5 cable, 100Base-Tx Full Duplex Mode

\section{Ethernet PHY Evalulation 2}

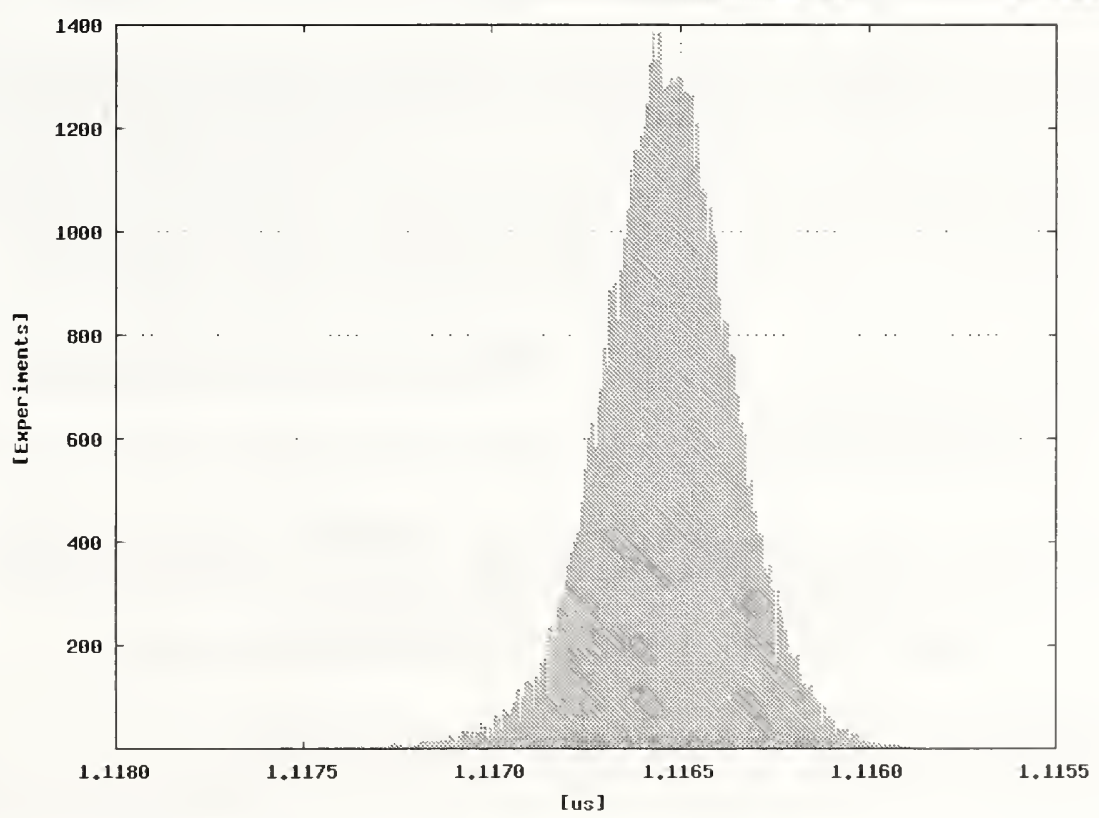

Jitter of 100.000 clock synchronization packets for

Gigabit Ethernet PHY's, 99m CAT-5 cable, 100Base-Tx Full Duplex Mode 


\section{Ethernet PHY Evalualtion 3}

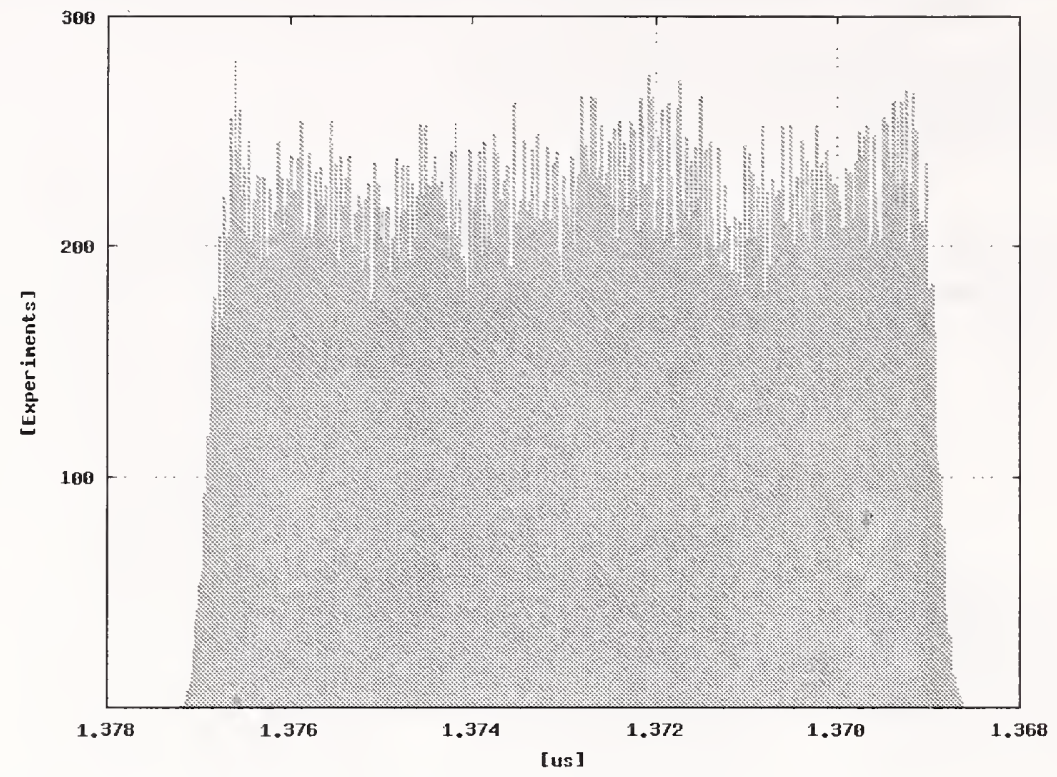

Jitter of 100.000 clock synchronization packets for

Gigabit Ethernet PHY's, 99m CAT-5 cable, 1000Base-Tx Full Duplex Mode

\section{Ongoing and Future Work}

- Thorough evaluation and measurements of hardware IP and protocol stack with the PSynUTC/IEEE 1588 prototype system

- Integration in SoC embedded systems (REMPLI, ...)

- Close cooperation with IEEE 1588 committee (1st IEEE 1588 workshop, ...)

- Application to sensor networks 


\section{Clock Synchronization Constraints.}

- How to achieve 10 ns accuracy ?

- Overall accuracy $\pi$ depends on

$$
\pi=c_{1} \varepsilon+c_{2} G+c_{3} u+c_{4} P \rho
$$

- $\varepsilon$... Transmission delay uncertainty

- G ... Local clock granularity

- $u$... Rate synchronization uncertainty

- Timing error due to discrete rate adjustment $\left(u=1 / f_{\text {osc }}\right)$

- P $\rho$... Clock drift during a re-synchronization period

$=\mathrm{P}$... length of the re-synchronization period

- $\rho$... oscillator drift

\section{Local Clock Granularity}

- Compensated external crystal oscillator

TCXO, MXCO, OXCO

- Limited frequency range: $1 \mathrm{MHz}$ up to $10 / 20 / 100 \mathrm{MHz}$

- PLL to decrease granularity

- On-Chip module $\times 4, \times 8 \ldots$

- Operating frequency of 96 bit accumulator

- Pipe-lined architecture

- > $200 \mathrm{MHz}$ using state-of-the-art FPGA families

* $>400 \mathrm{MHz}$ using affordable CMOS-ASIC fabrication processes

- Generic adder based clock generator module - Different optimization criteria 


\section{Mbit/s Transmission Delay Uncertainty}

20000

$$
\sigma \approx 300 n s
$$

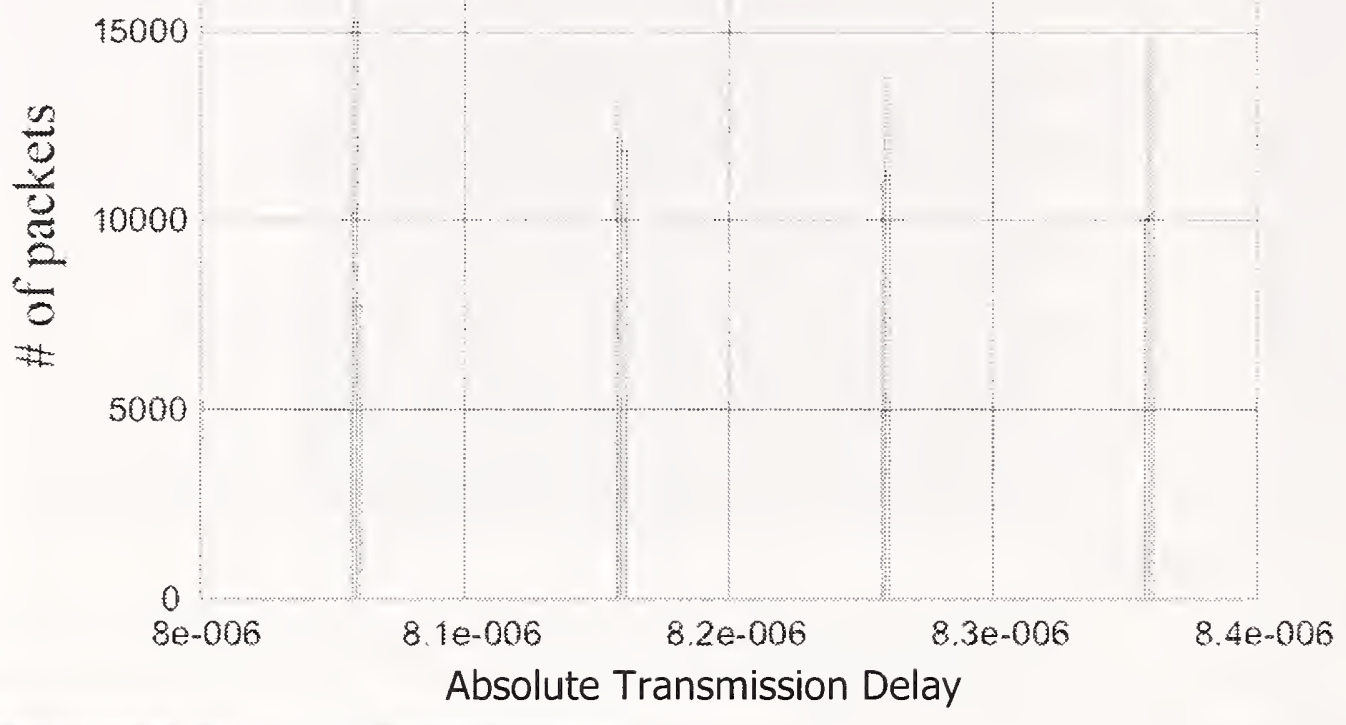

\section{Re-synchronization Mechanism}

- Interval clocks instead of ordinary clocks

- Local clock values are adjusted

- Accuracy intervals are adjusted

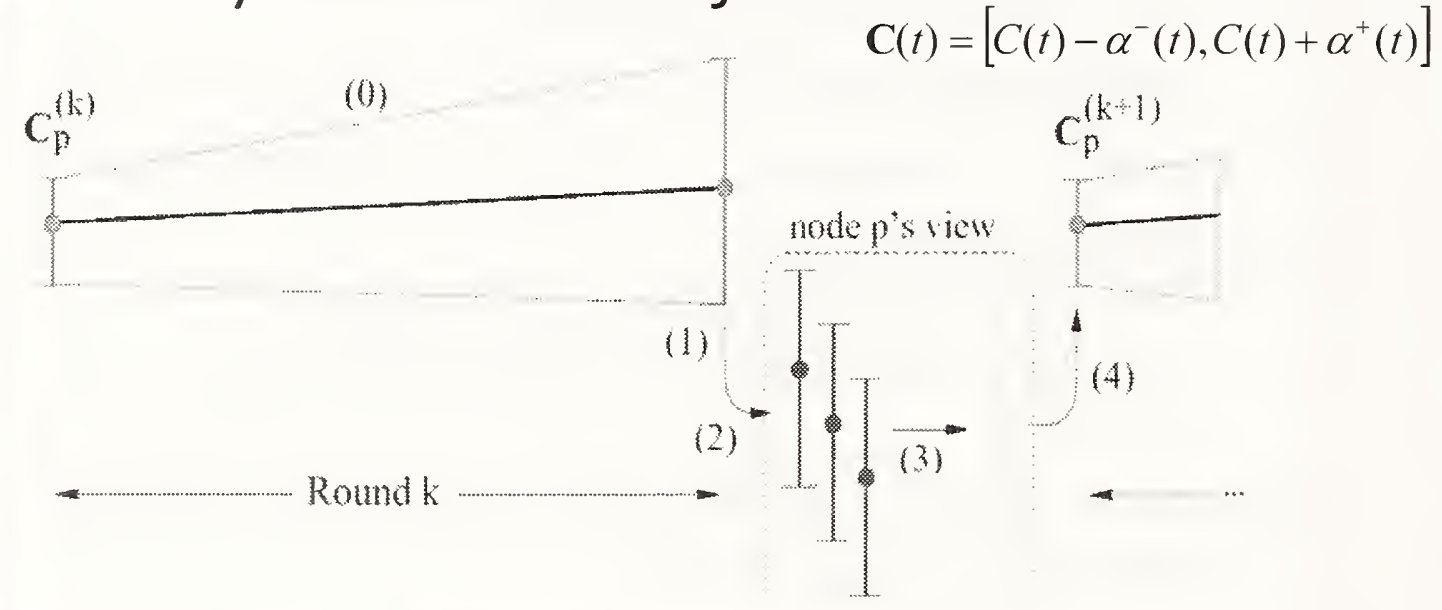

$$
\mathbf{C}(t)=\left[C(t)-\alpha^{-}(t), C(t)+\alpha^{+}(t)\right]
$$

$C_{p}$

(1) 


\section{Mbit/s Transmission Delay Uncertainty}

2000

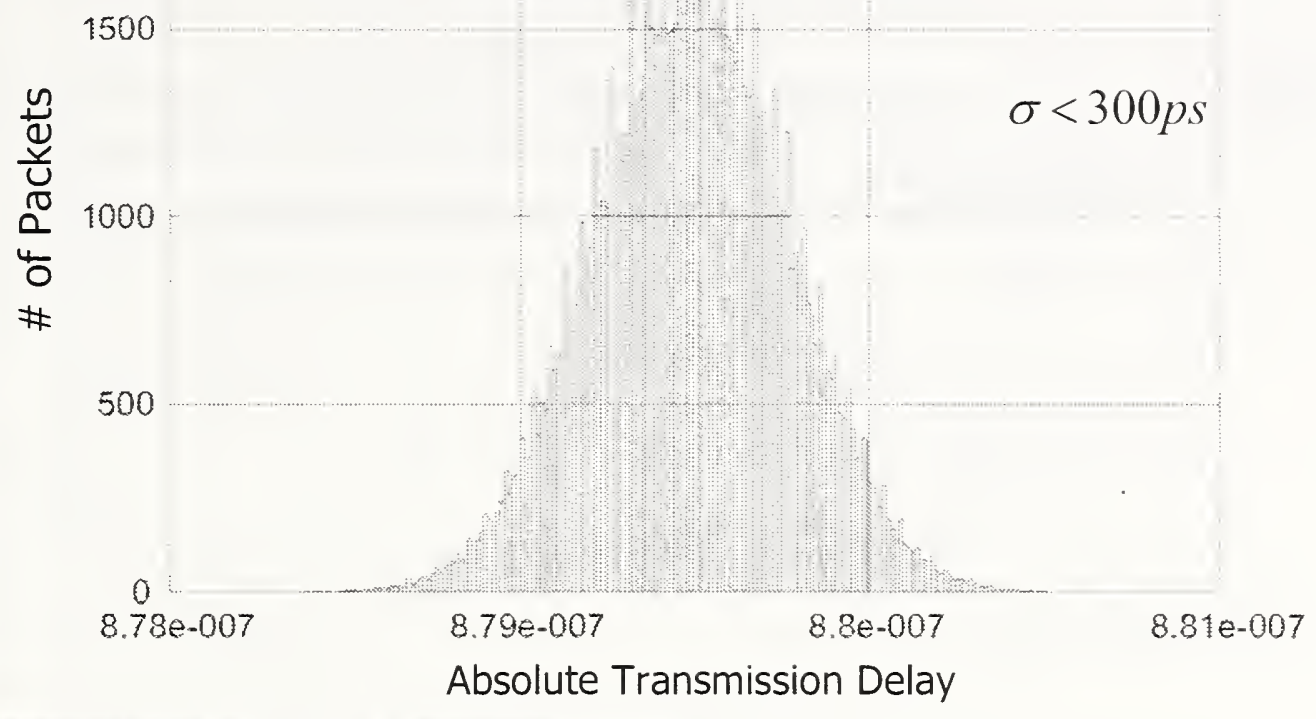




\section{PTP in redundant network structures}

\section{Topics}

- Configurations

- Recovery

- Proposed enhancements

- Time Master Redundancy

- Secondary Master Handling

- Conclusion

\section{Configurations}

- A redundant system has at least two nodes that are connected by at least two different communication paths

- Ring structure as base for redundancy is the most common type of architecture

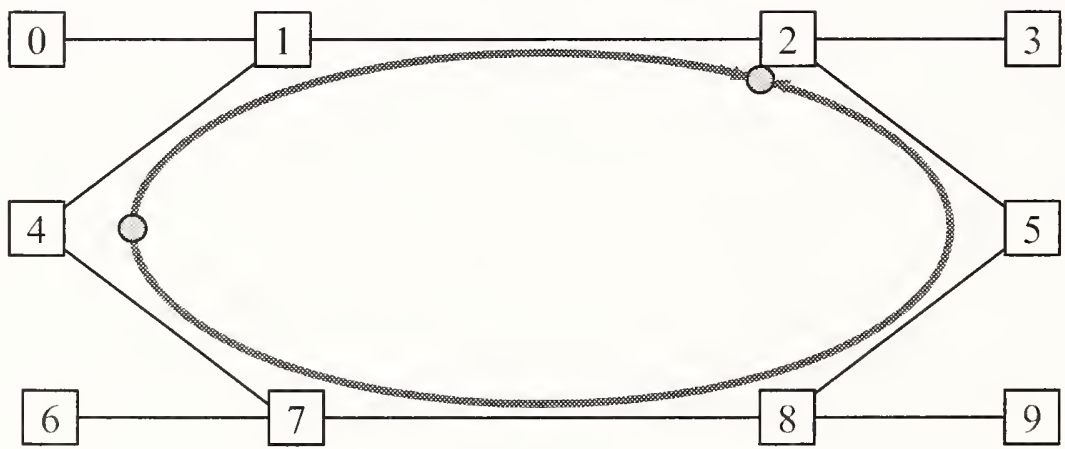

$>$ delays between 2 nodes are different ...PTP must know path See path between 4 and 2 via 1 and via 7,8,5 


\section{configuration issues}

- Circulating frames shall be avoided in switched networks

- Every ring node shall be something like a boundary clock

- As only one port shall be PTP slave in a Boundary Clock, the ring has to be broken up at some place

Example: node 0 is master clock

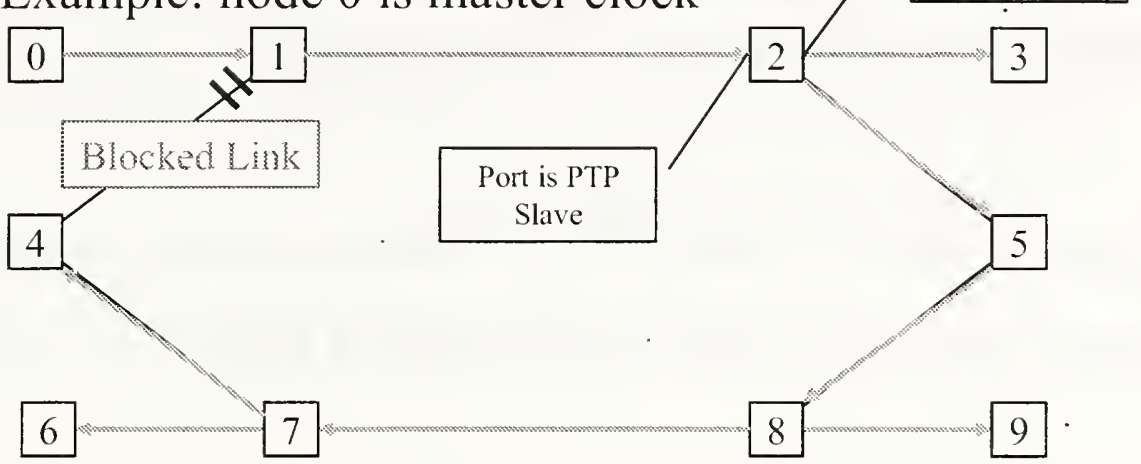

Page 3

\section{Error Recovery}

- Assume a link error between node 1 and 2

- Blocked link will be opened and 1 will send time synch to 4

- The Boundary clocks 2,5,8,7 continue to send synch

- Timeout occurs at node 2 first, he will change the clock state

- 5, 8, 7,4 will follow after additional time outs (some messaging)

- Beginning from node 4 the ring will be resynchronized $(7,8,5,2)$

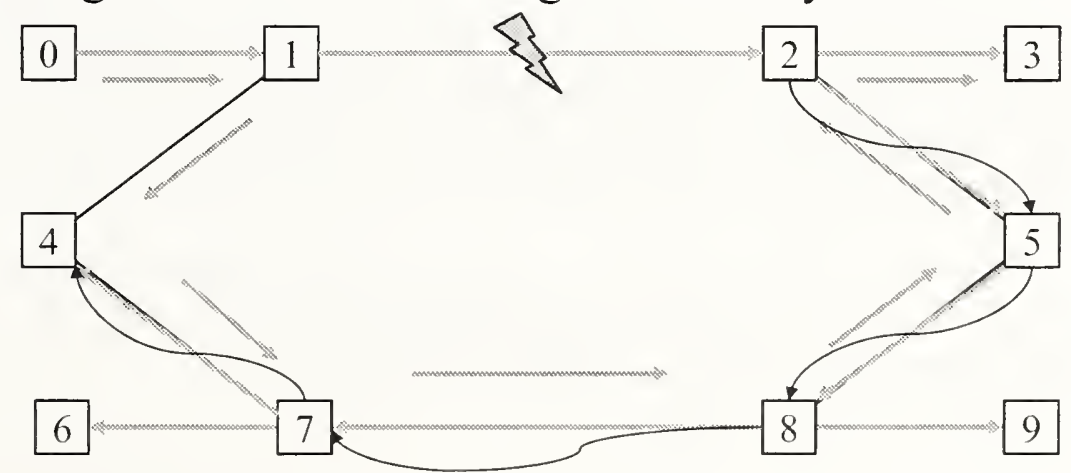

Page 4 


\section{Discussion of recovery}

- Most actions will be executed sequentially

- This will lead to not acceptable delay

- Management messages may improve performance

... but with a lot of sophisticated protocols

- Blocking ports result in a poor recovery time

Potential enhancements

- Redundant path may improve accuracy if used

\section{Proposed enhancement in PTP}

- Use both paths (red and blue)

- Use the switches that forward time with compensated delay instead of boundary clocks

- The follow up messages require a unique transaction identifier (stations that split messages shall produce two distinct transaction identifier in sync and follow up)

- Option: Average timing errors in each end node receiving from the same node on different paths 


\section{Time Master Redundancy}

- Time Masters in stand by will produce the same effects as blocked ports

- Boundary clocks in switches will block a second master

- Resynchronization will lead to instable conditions (each control loop has to adopt to new drift parameters)

- All happens sequentially which makes change slow

- Simultaneous adoption to new master clock required

- Same principles apply as shown before

\section{Secondary Master Handling}

- Switch uses best master clock algorithm

- Only the best master clock will be forwarded

- Delay measurement will be done on any link and is available on both sides (otherwise a delay measurement cycle is needed)

- At time out of the current best master clock the switch that has both as sources will forward the messages from the second master clock

- The end nodes work as specified by IEEE 1588 best master clock algorithm 


\section{Example: Node 0 and 4 are Master Clocks}

\section{0 is best Master Clock}

- Error in Master Clock 0

- 1-5 detects timeout

- 5 will forward master clock 4

- Link 1-3 and 3-5 will forward in reverse direction

- Delay parameter are unchanged

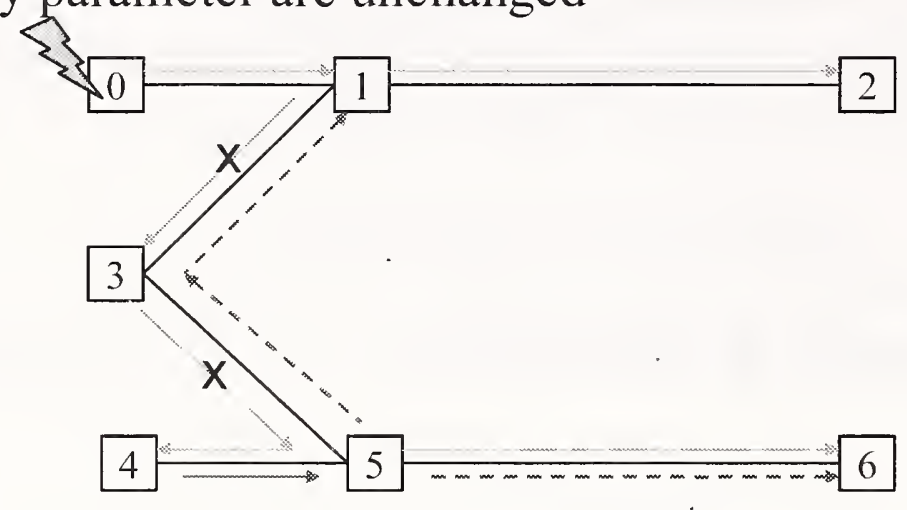

Page 9

\section{Conclusion}

- We propose to enhance IEEE 1588 for redundancy!

- The approach is in accordance with other requirements of a switched network for sub microsecond accuracy

- A technical proposal is under discussion within IEC SC65C REAL TIME Ethernet 


\section{A Solution for Fault-Tolerant IEEE-1588}

Jeff Allan and Dr. Dongik Lee

Dependable Real-Time Systems Ltd, U.K.

\section{Introducing DRTS Ltd}

$\square$ Dependable architecture and solutions for safety critical distributed embedded systems:

- Dependable CAN on the basis of reliable and accurate global time reference

$\square$ This presentation outlines:

- A fault-tolerant clock synchronisation technique;

- Its application to IEEE1588 


\section{Synchronisation Methods}

口Hardware based

- Nanoseconds precision, inflexible, expensive

口Hybrid approach

- Microseconds precision, cost-effective

$\square$ Software based

- Milliseconds precision, flexible, low cost

\section{Synchronisation for Embedded Systems}

$\square$ Common features of embedded system synchronisation

- Low cost and Low overhead

- Low bandwidth and computing resources

$\rightarrow$ Software approach with Master-Slave structure

$\square$ For safety-critical embedded applications

- High precision (microseconds)

- Fault-tolerance

$\Rightarrow$ Multiple-master 


\section{Software-Based Algorithms}

Message latency causes the major problem with software-based approach
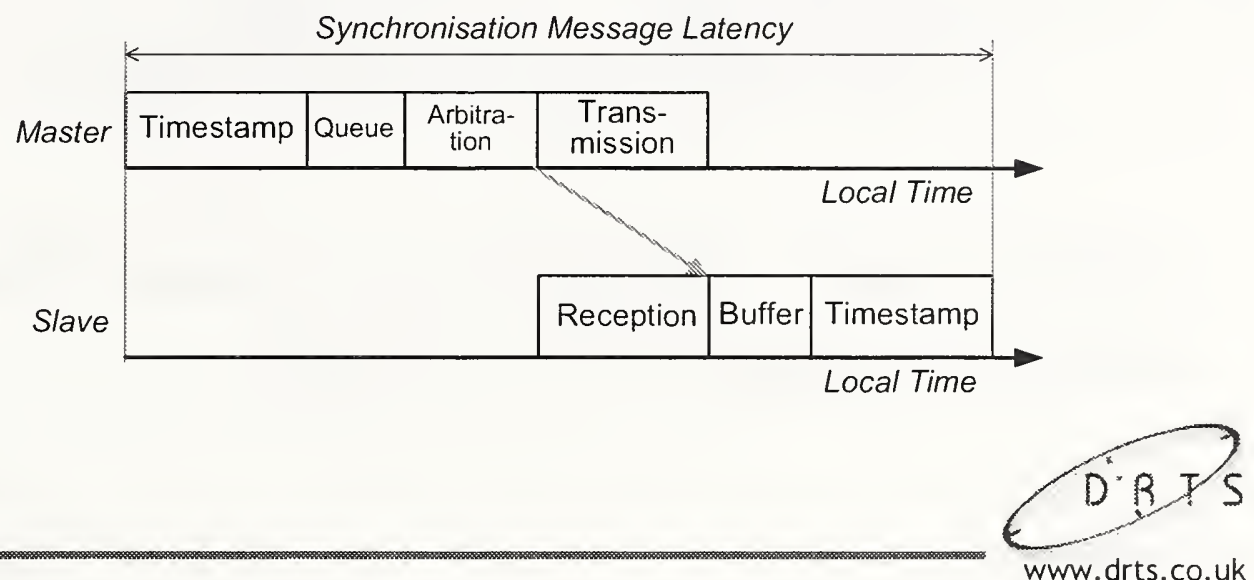

\section{A Posteriori Technique (1)}

$\square$ A software synchronisation method with microseconds precison for CAN network*

- To reduce the influence of message latency and jitter

- Master and slaves take timestamps at the end of messag transmission (or reception)

*Gergeleit \& Streich (1994). "Implementing a distributed high-resolution real-time clock using the CAN bus", Proc. $1^{\text {st }}$ iCC.

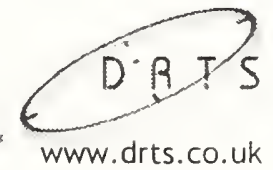




\section{A Posteriori Technique (2)}

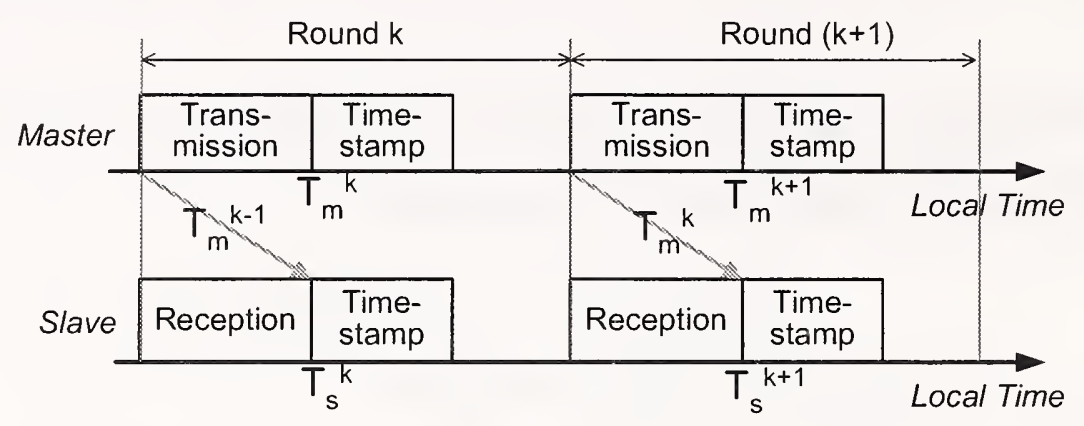

$\square$ Clock skew $\approx 1$ bit time (e.g., $1 \mu$ s at $1 \mathrm{Mbps}$ ) $\square$ Fault-tolerance?

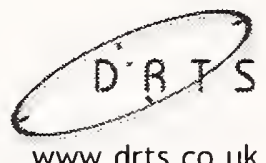

\section{Multi-Master Techniques}

A multi-master approach needs a complex voting mechanism

$\square$ Complexity and necessary bandwidth increases with the number of master clocks

$\Rightarrow$ A DRTS solution to:

$\square$ Minimise voting complexity, bus load, and use of computing resources

$\square$ Maximise fault-tolerance and design flexibility. 


\section{DRTS Technique to Achieve Fault-Tolerance}

$\square$ Classifying the clocks into three groups
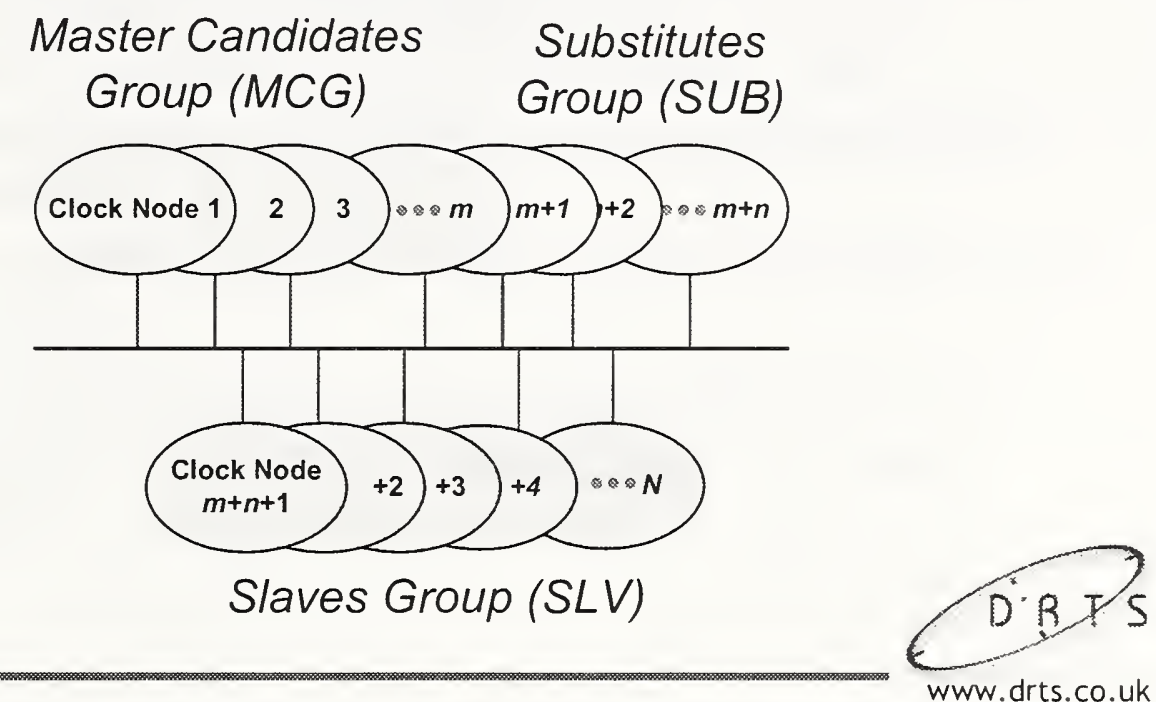

\section{Replacing Faulty Candidates}

$\square$ Voting takes place only in MCG

$\square$ Clocks in SUB replace any faulty clocks in MCG

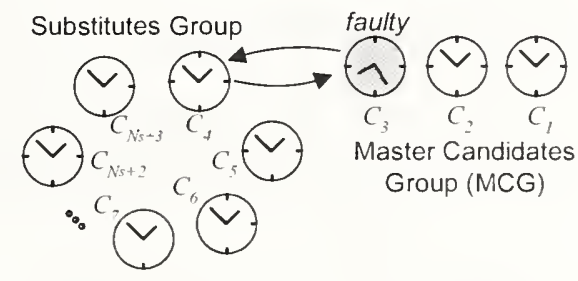

The size of MCG: $N_{m}=2 f_{m}+1$ (or $3 f_{m}+1$ to tolerate Byzantine faults)

$\square$ The size of SUB: $N_{s}=2 f_{m}$

$\square$ The level of fault-tolerance is mainly dependent on $N_{S}$ $\rightarrow$ The voting complexity is minimised

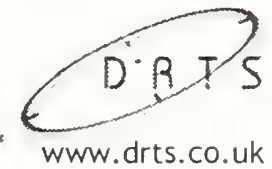




\section{Master Selection and Synchronisation}

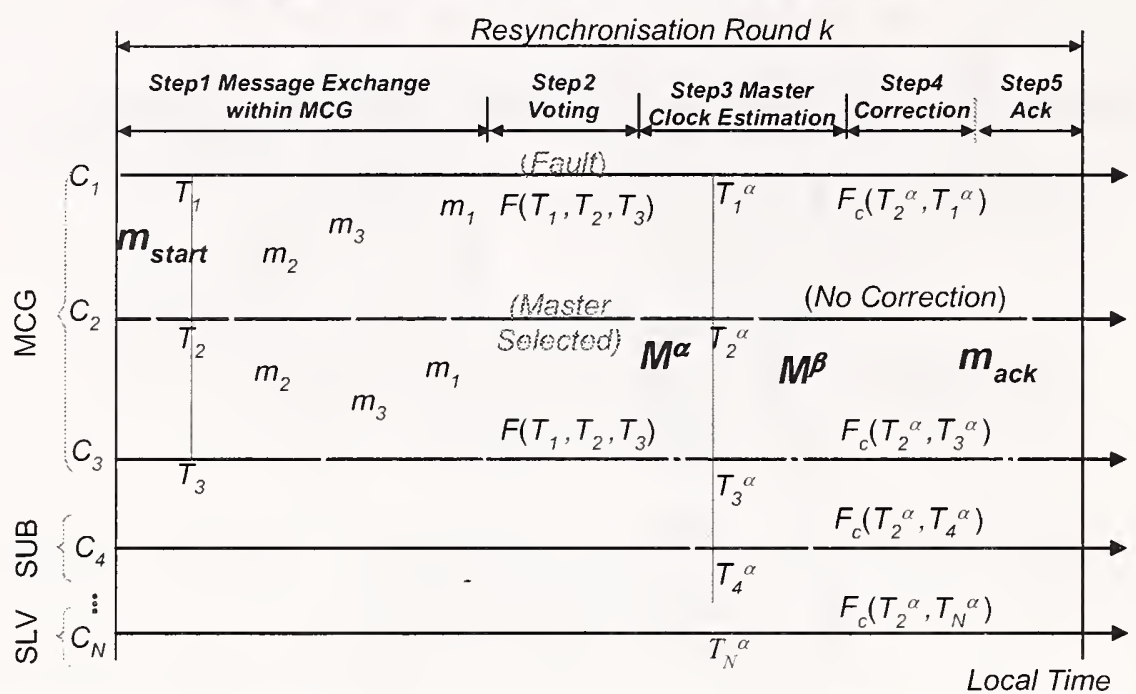

In this example:

- Size of MCG: $N_{m}=3$ (i.e. $f_{m}=1$ ) - Size of SUB: $N_{s}=2$

$-C_{2}$ is the best clock

- $C_{1}$ is a new faulty clock

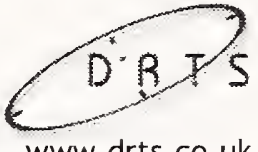

\section{Synchronisation Messages (1)}

$\square$ Start Message $\left(m_{\text {start }}\right)$

- Fastest clock(s) starts a new synchronisation round: Starting clocks are not necessarily the best clock

$\square$ Timestamps $\left(m_{i}\right)$ exchange within MCG clocks 


\section{Synchronisation Messages (2)}

$\square$ Two successive Sync Messages $\left(M^{\alpha}, M^{\beta}\right)$ :

- $M^{\alpha}$ for the list of MCG members

- $M^{\beta}$ for the master clock timestamp

- In the next round, different clock can be selected as master

- Immediately detect a fault of 'selected master' and re-elect

$\square$ Acknowledgement Message (mack) by a substitute: Accept or Reject to be a new member of MCG

\section{Analysis}

$\square$ Synchronisation precision:

- Worst clock skew: $\delta=4 \rho R+\xi$

Where, $\rho=$ drift rate, $R=$ resynchronisation period, $\xi=$ reading error (including 1-bit time and interrupt processing)

$\square$ Number of messages for tolerating $f_{m}$ faulty candidates in a single synchronisation round:

- $2 f_{m}+4 \leq n_{m s g} \leq(n+2) f_{m}+4, \quad(n=1,2, \ldots)$

(e.g.) Bandwidth of CAN used for synchronisation $\left(f_{m}=1 ; n=2 ; N_{s}=2 ; N_{m}=3\right):<0.1 \%$ at $1 \mathrm{Mbps},<0.4 \%$ at $250 \mathrm{Kbps}$ 


\section{Experiments with CAN}

$\square$ Steer-by-wire model system and demonstrator
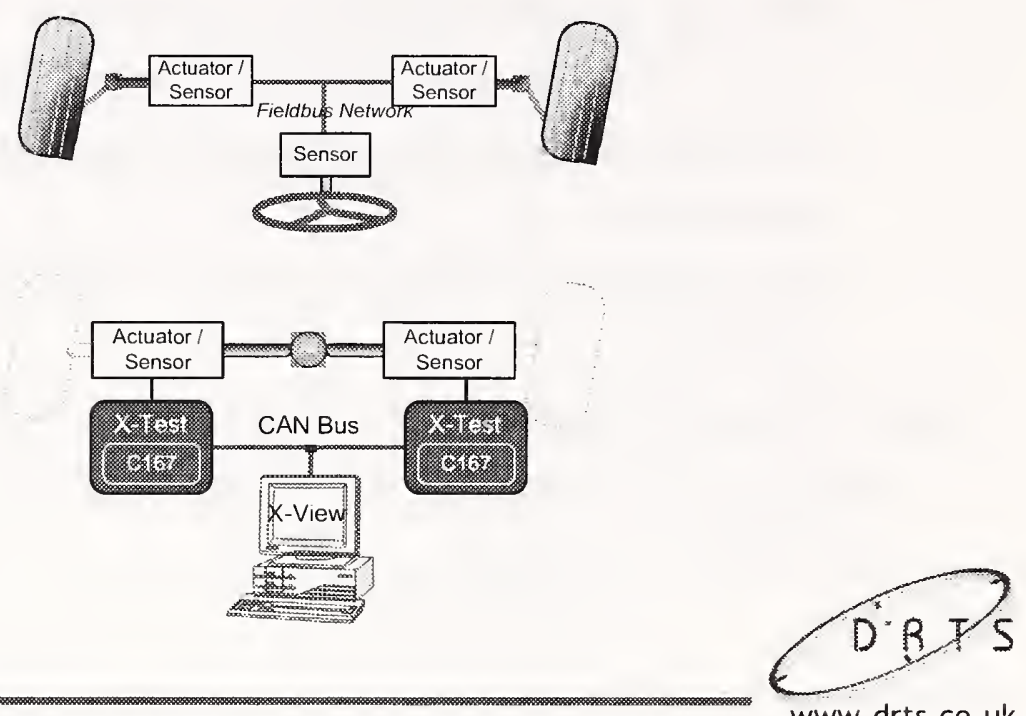

www.drts.co.uk

\section{Steer-By-Wire Demonstrator}

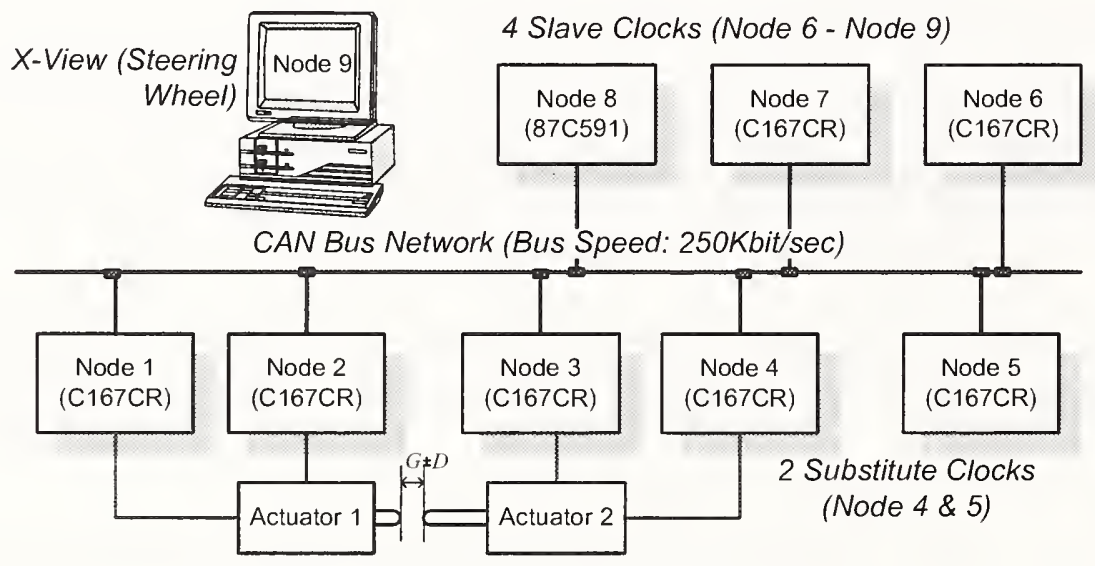

3 Master Candidate Clocks (Node 1 - 3) and 2 Actuators

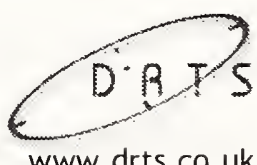




\section{Transitions of Clock Status}

$\square$ Clock status changes when the clocks in MCG are reset by manually
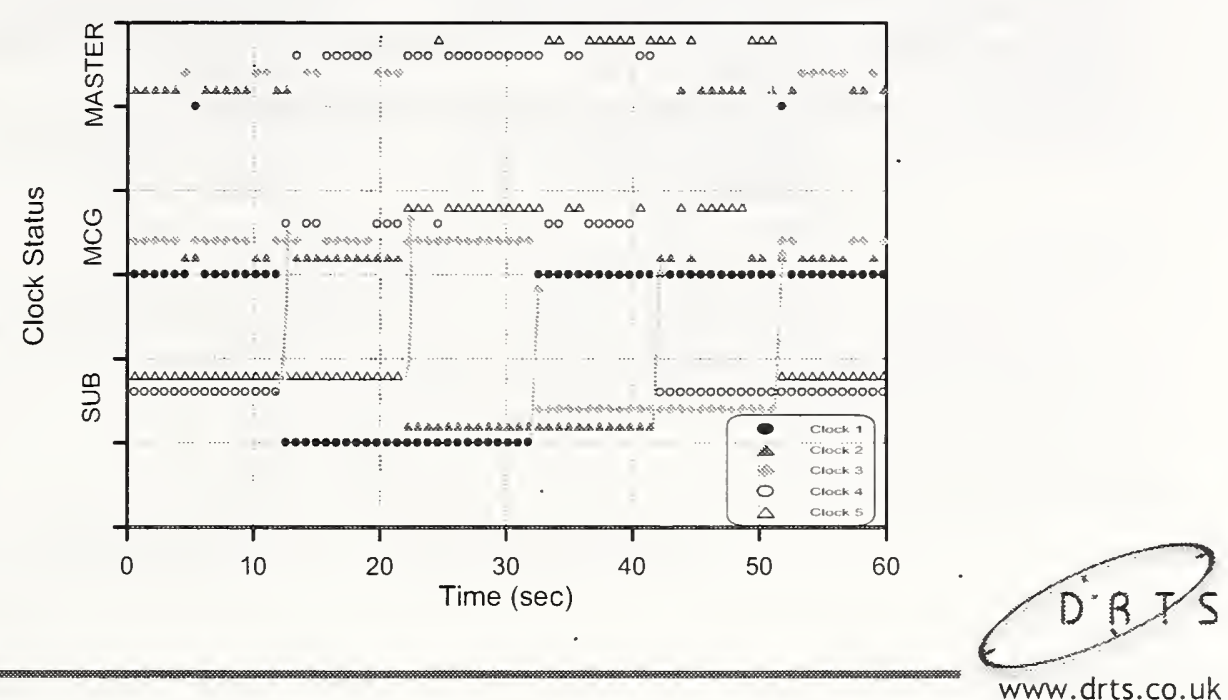

Control Performance

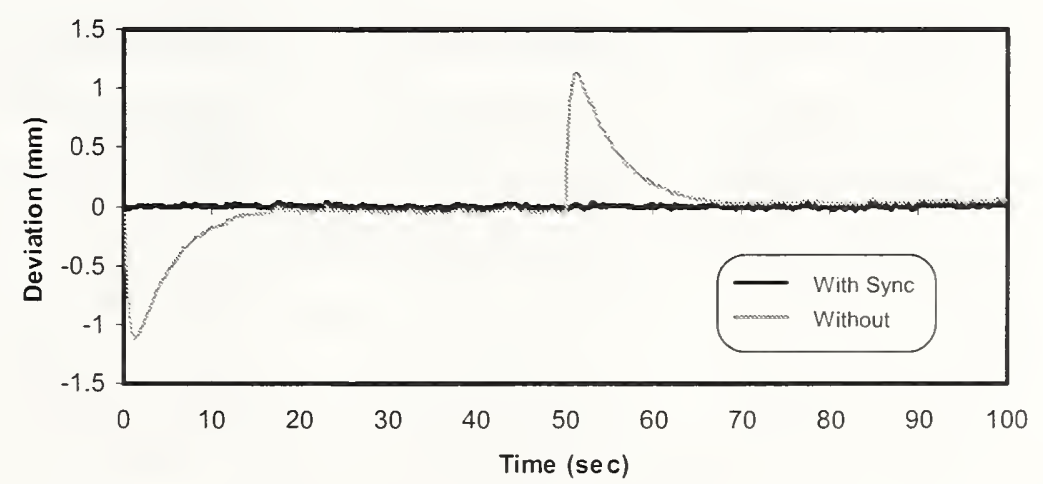

Deviation between two actuator strokes with/without clock synchronisation (background bus load level $90 \%)$

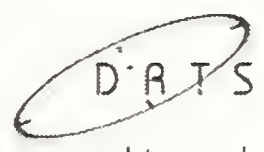




\section{IEEE1588: An Ideal Platform for DRTS Synchronisation}

\begin{tabular}{|c|c|c|}
\hline & IEEE1588 & DRTS Synchronisation \\
\hline Network & Multicast & Multicast \\
\hline Optimised for & Embedded systems & Embedded systems \\
\hline Category & Hybrid & Software \\
\hline Structure & $\begin{array}{l}\text { Master-slave (Single } \\
\text { master) }\end{array}$ & $\begin{array}{l}\text { Master-slave (Multi } \\
\text { master+voting) }\end{array}$ \\
\hline Precision & Nanoseconds & Microseconds \\
\hline $\begin{array}{l}\text { To overcome } \\
\text { message latency }\end{array}$ & $\begin{array}{l}\text { Special hardware }+ \\
\text { a posteriori technique }\end{array}$ & A posteriori technique \\
\hline Fault-tolerance & No & Yes \\
\hline
\end{tabular}

Ideal platform $\Leftrightarrow$ Fault-tolerance

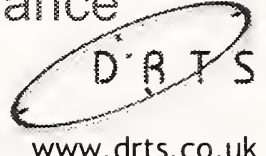

\section{A Solution to Fault-Tolerant IEEE1588}

Substitutes Group

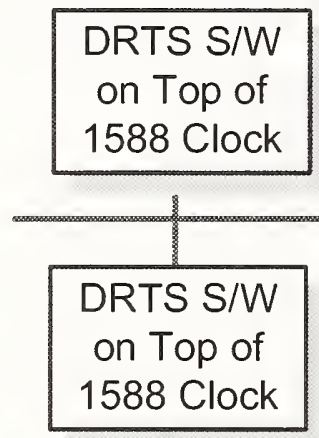

Slave Clocks Group

\begin{tabular}{|c|}
\hline DRTS S/W \\
on Top of \\
1588 Clock \\
\hline
\end{tabular}

DRTS S/W
on Top of
1588 Clock

DRTS S/W on Top of 1588 Clock

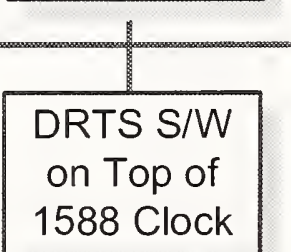

Master Candidate Clocks Group

$\rightarrow$ Fault-tolerant time reference with nanoseconds precision without hardware modification or extra hardware

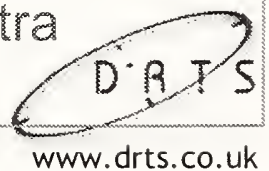




\section{Improvements to DRTS Synchronisation}

$\square$ DRTS synchronisation requires for $\mathrm{CPU}$ interrupt signals that are not always available.
口 The IEEE1588 clocks with special hardware provides nanoseconds precision without CPU interrupts.
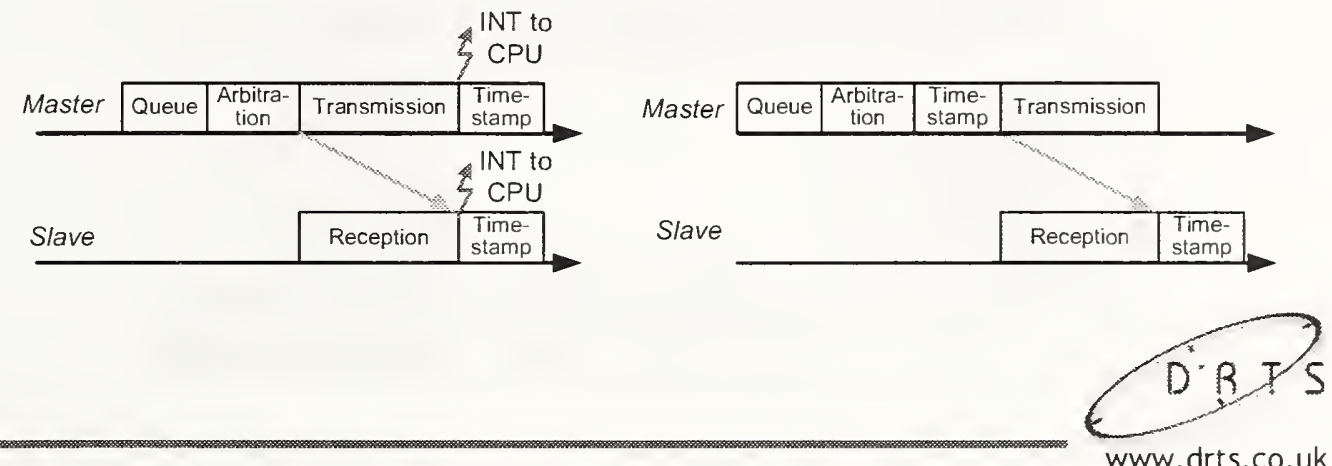

\section{Multi-Subnet Systems and Heterogeneous Clocks (1)}

Synchronisation within each sub-net

$\square$ If entire network is initially synchronised, synchronised clocks within each sub-net also synchronise to the rest of sub-nets*.

$\square$ GPS receiver may be a member of MCG.

$\square$ Boundary clocks can be a grand master

$\rightarrow$ Clocks within each subnet remains synchronised in the presence of faults in boundary clocks, routers, repeaters, or GPS receivers. 


\section{Multi-Subnet Systems and Heterogeneous Clocks (2)}
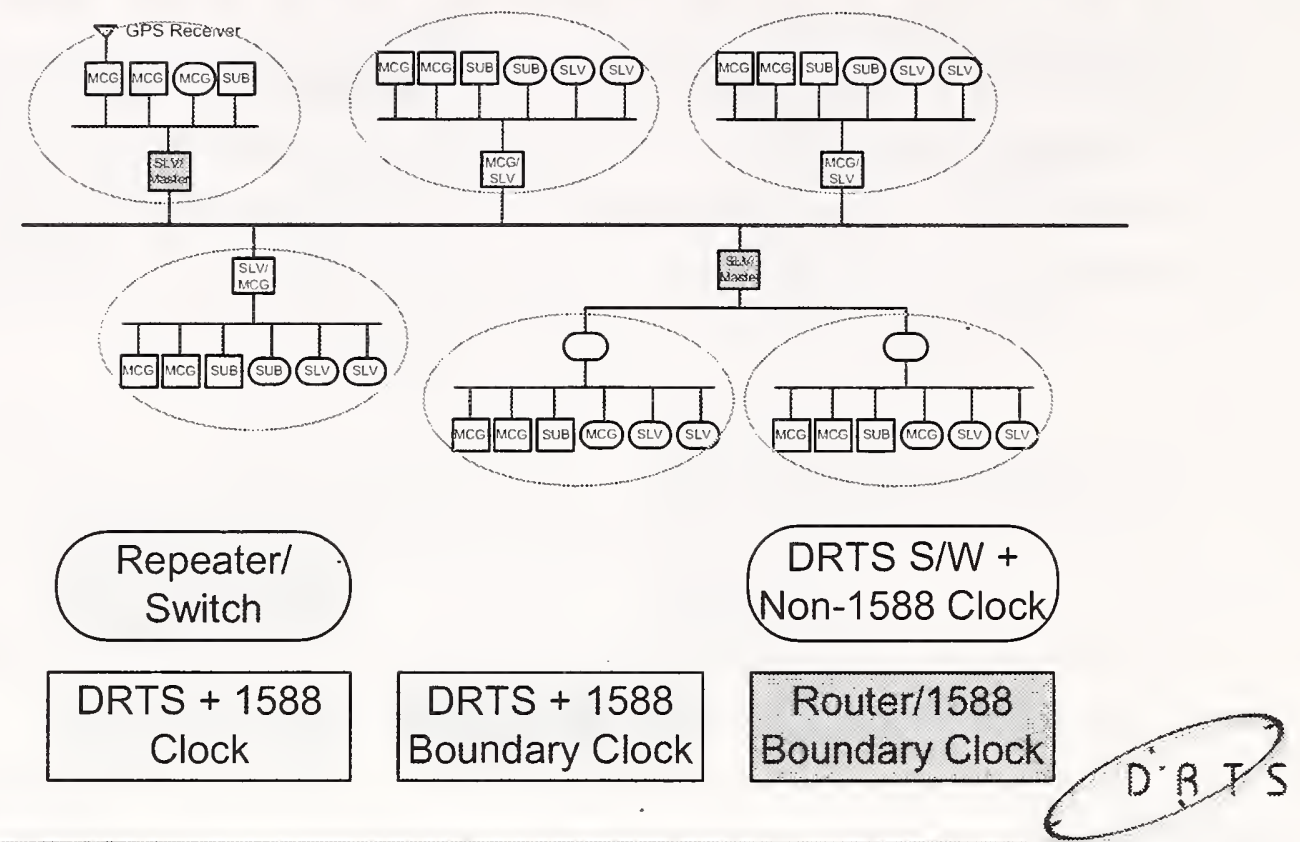

www.drts.co.uk

\section{Summary and Conclusion (1)}

$\square \mathrm{A}$ unique clustering technique has been presented:

- Software based technique-Low cost, No need for additional hardware

- Minimise voting process-Simplicity and efficiency

- Flexibility $-N_{s}$ and $N_{s}$ can be chosen as design factors

- Deterministic-Achievable fault-tolerance and bandwidth used can be predicted

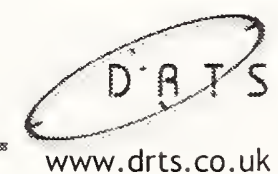




\section{Summary and Conclusion (2)}

QDRTS technique can provide fault-tolerance clock synchronisation with IEEE1588

- IEEE1588 provides ideal foundation for the DRTS approach

- Software technique $\rightarrow$ No need for hardware modification

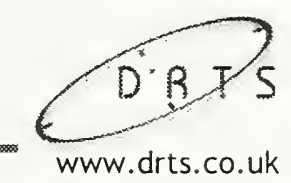




\title{
A Solution for Fault-Tolerant IEEE 1588
}

\author{
Jeff Allan and Dongik Lee \\ Dependable Real Time Systems Limited \\ The Innovation Centre, 217 Portobello Road, Sheffield, S1 4DP, United Kingdom \\ Phone/Fax: +44-(0)1142232301 \\ E-mail:jeff.allan@drts.co.uk \\ Website: www.drts.co.uk
}

\begin{abstract}
A software-based fault-tolerant clock synchronisation technique is presented. The proposed algorithm aims at fault-tolerant clock synchronisation within a subnet involving any number of heterogeneous clocks with or without IEEE1588 clocks. The proposed algorithm is simple, and provides predictable and reliable time references in the presence of faults with grandmaster or network connections.
\end{abstract}

\section{INTRODUCTION}

Clock synchronization is a key factor for many industrial systems. For example, synchronised clocks are the fundamental requirement for embedded control systems, time-triggered communications, redundancy management, etc. The IEEE 1588 standard [1], or PTP (Precision Time Protocol), provides a precise clock synchronisation protocol for multicast networks. In contrast to NTP (Network Time Protocol), IEEE1588 aims at measurement and control applications and the protocol can provide system-wide synchronisation precision in the submicrosecond range. However, the potential drawbacks of PTP are a lack of capability to tolerate faulty master clocks and a selection mechanism that is probably too complicated (and expensive) to be used in low-cost embedded applications.

This paper presents a software-based master selection mechanism that will tolerate faulty master clocks, and also maintain synchronisation in any IEEE1588 subnet, low-cost or otherwise. The proposed algorithm was originally developed for CAN (Controller Area Network), which is also a multicast network. The proposed algorithm deterministically guarantees an upper-bound for the clock skew and the message overhead.

\section{IEEE-1588 FOR LOW-COST EMBEDDED SYSTEMS}

DRTS Ltd has been developing dependable architecture and solutions for safety-critical embedded systems such as "x-by-wire" applications for vehicles. The fundamental requirement for a dependable distributed architecture is a precision system-wide time reference. From the point of view of synchronisation precision, the IEEE1588 standard may provide an ideal platform for establishing dependable systems architecture. However, for low-cost embedded systems including safety-critical applications, there are several potential drawbacks:

- The mechanism for selecting the master clock is complex and represents "overkill" for use in low-level embedded applications;

- The behaviour of faulty clocks is assumed as "fail-silent" - that is, a new master will be selected when the current master does not send a "sync" message in time. However, if the current master is faulty, so that it gets faster, this scenario may not be detected by other clocks, and the healthy slave clocks may claim themselves faulty; and also,

- The desired number of IEEE1588 clocks with the stratum number 1 or 2 (i.e., GPS 
receivers or atomic clocks) may not be available.

For example, considering an IEEE1588 " $\mathrm{x}$ by-wire" implementation typifying a low-cost embedded application, a single GPS receiver would be available, and the rest of nodes in the system would be likely to have lower quality clocks. Most likely, many of the nodes would not have IEEE1588 clocks. In this scenario, the PTP "best master clock" algorithm would be unable to tolerate a faulty master. In addition, because the standard allows only a single path to each subnet, a system could be left without synchronisation resulting in loss of messages or a disconnected communication.

\section{THE "A POSTERIORI" TECHNIQUE FOR CAN}

The communication network on which the DRTS approach is based is standard CAN. The precision that a software-based synchronisation method can achieve is limited by message latency and jitter. The result would be a typical precision in the order of a few milliseconds.

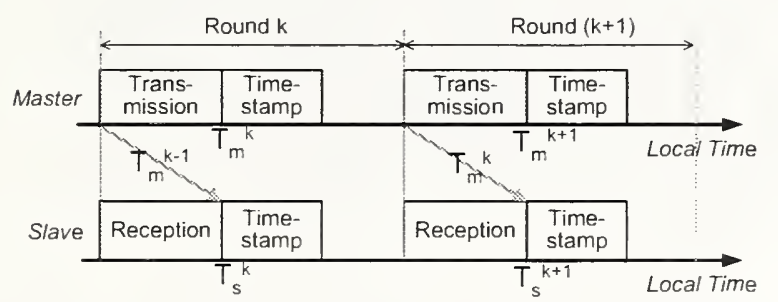

Figure 1. Clock synchronisation based on the "a posteriori" technique.

To overcome the message latency problem, Gergeleit and Streich [2] proposed a clock synchronisation technique based on " $a$ posteriori" technique (see figure 1). A clock in the system is designated as the master, which periodically broadcasts a synchronisation message that provides a reference time value. In this method, timestamps are taken right after a message is delivered, rather than before broadcasting the message. In the synchronisation round $\mathrm{k}$, the master broadcasts a synchronisation message $m^{k}$ which contains its timestamp taken at $T_{m}^{k-1}$ when the previous synchronisation message $m^{k-1}$ was delivered to the slaves. Every slave in the system simultaneously receives this message at $T_{s}^{k}$, and takes a timestamp right after the reception. Each slave clock then calculates a correction term using the difference between the timestamps $T_{s}^{k-1}$ and $T_{m}^{k-1}$. The key advantage is the high precision that does not depend on message latency. This approach can achieve the synchronisation precision up to 1

microsecond. However, the lack of capability to tolerate a faulty master is the major concern.

\section{FAULT-TOLERANT CLOCK SYNCHRONISATION FOR CAN}

DRTS has developed a unique mechanism to provide the "a posteriori" technique with fault-tolerance capability. This section describes the fault-tolerant clock synchronisation algorithm for CAN. The application of this technique to any broadcast networks having IEEE1588 clocks will be discussed in section 5 .

The key feature of the proposed algorithm it the use of dynamic voting within a set of master candidate clocks. The novelty and value of the method lies in the use of two groups of master candidates-'Master Candidates Group' (MCG) and 'Substitutes Group' (SUB). By classifying the candidate clocks into two groups, the complexity and bus traffic of the voting mechanism can be drastically reduced.

\subsection{Subsets of Clocks in the System}

The major drawback with a multiple-master technique is the need for a master selection mechanism. Selection algorithms are usually complicated and introduce extra load on the system in terms of bus traffic and processing time. The number of messages required for the selection mechanism increases with the 
size of the multiple-master cluster. The larger size of the multiple-master also leads to the higher complexity in the selection mechanism, which is not desirable.

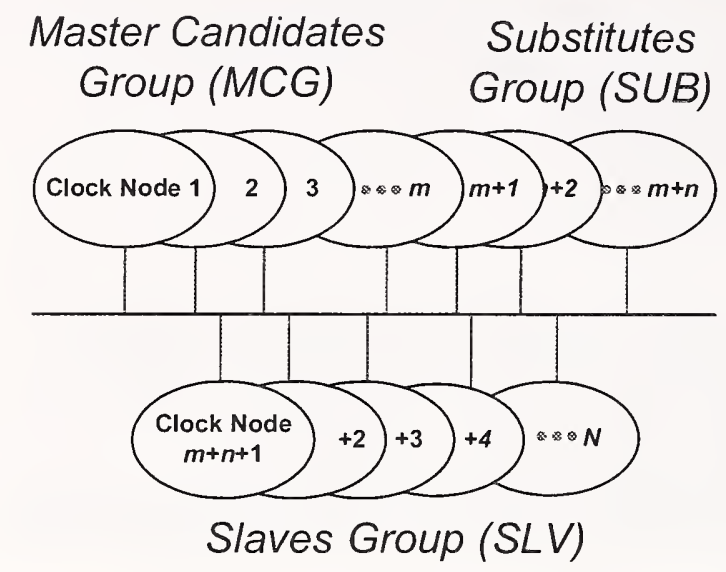

Figure 2. Subsets of clocks in the system.

In this paper, to overcome these problems, all clocks in the system are divided into three subsets (figure 2). At each synchronisation round, only clocks in the MCG take part in voting for a master. Clocks in the SUB group do not take part in the voting process, and are only for replacing faulty members of the MCG. The rest of the clocks in the system are considered to be slaves (SLV).

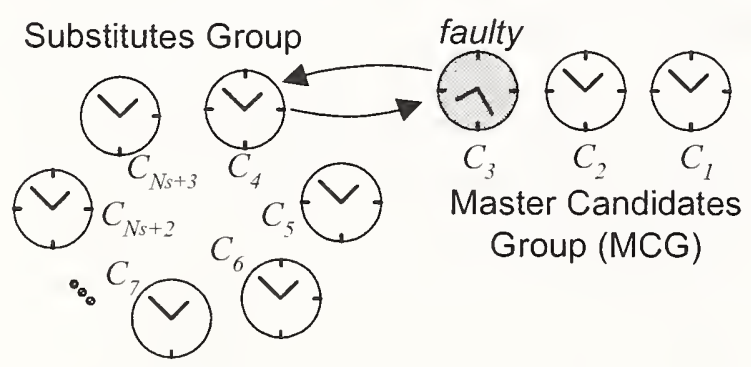

Figure 3 . Replacement of a faulty candidate clock.

An example given in figure 3 explains how the MCG and the substitutes group work in order to update the MCG. Each node of the MCG examines its clock value by comparing with the current master clock time. When a clock in the MCG is found to be faulty, a nonfaulty clock in the SUB group becomes a new member of the MCG. In figure 3, for example, the faulty candidate $\mathrm{C}_{3}$ is replaced with $\mathrm{C}_{4}$. Clock $\mathrm{C}_{3}$ takes the place of $\mathrm{C}_{4}$, rather than being removed from the system. The number of clocks for each group can be chosen by the system designer to achieve the desired level of fault-tolerance. The size of MCG to tolerate $f_{m}$ faults is given by

$$
N_{m}=2 f_{m}+1
$$

If Byzantine faults are assumed, the size of MCG group is given by $N_{m}=3 f_{m}+1$. Note that $f_{m}$ denotes the maximum number of new faulty clocks of the MCG that can arise in a single resynchronisation round. Thus, the complexity of the selection mechanism is not directly proportional to the total number of faulty clocks assumed in the system. On the other hand, the size of the substitutes group, $N_{s}$, depends on the total number of faulty clocks $(f)$ to be tolerated in the system. It seems useful to choose $N_{s}$ as a multiple of $f_{m}$, to achieve $\eta$-modular set of redundant clocks to substitute for faulty master candidates; that is,

$$
N_{s}=\eta f_{m}, \eta=0,1,2, \ldots
$$

The minimum size of SUB group is zero in the case that no clocks are designated to the SUB group. Since $f_{m}<<$, the proposed algorithm can achieve a desirable degree of fault-tolerance using a simple voting mechanism and a lower number of message exchanges.

\subsection{State Diagram Model for the Algorithm}

The state diagram in figure 4 is used to describe how the proposed synchronisation algorithm works. Circles and arrows represent states and transitions, respectively. In the resynchronisation process, clocks in the system can be in one of the states shown. Transitions from one state to another are triggered either on the reception of a message or by the expiration of a deadline. Conditions for the state transitions are shown on the upper part of the labels. The lower part of the label shows the content of message, which is 
sent at the time of the corresponding transition. A null label represents the condition that no message is sent or received.

RESYNC_DETECTION: The synchronisation round $k$ begins when any clock in the MCG reaches to $k R$ on its logical clock, where $R$ is the resynchronisation interval. In practice, the fastest clock, or even a faulty clock, in the MCG will reach a new synchronisation round at first, and will generate a start message.

MCG_TIMESTAMPS_EXCHANGE: This state is triggered on the reception of a start message. Each clock in the MCG takes its timestamp based on the "a posteriori" technique. Each candidate clock then broadcasts its timestamp to the other clocks, and also waits for messages from other candidate clocks. Either when all the timestamps are received, or when the deadline for the message exchanges is expired, each clock transits to the next state for selecting a master.

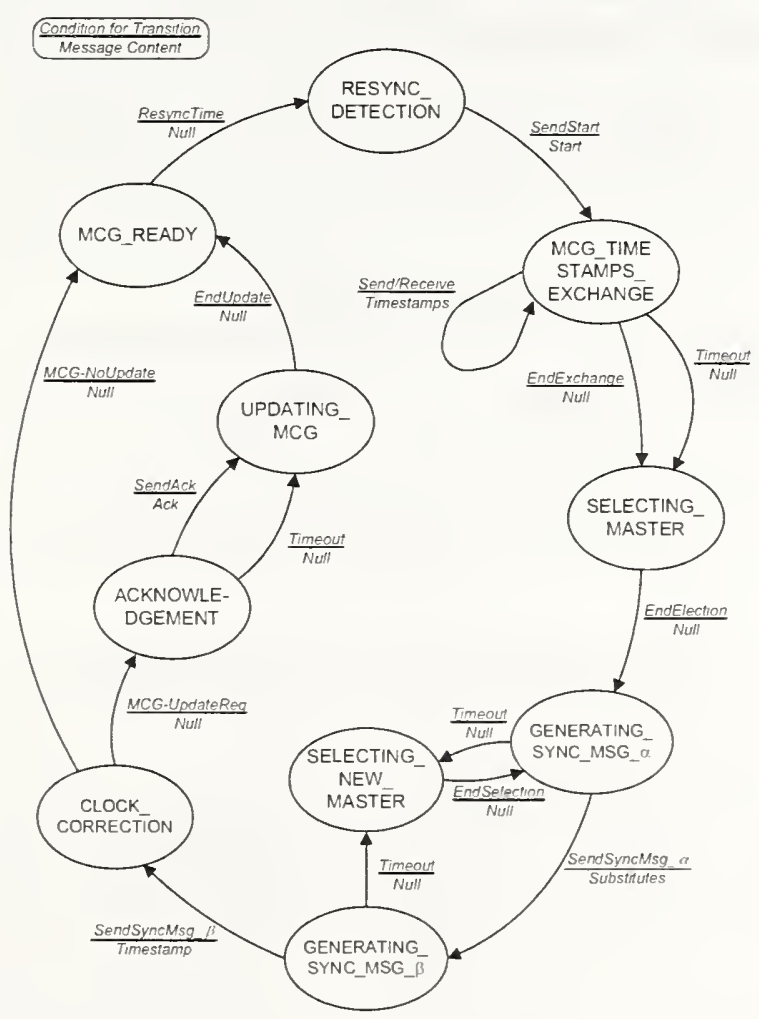

Figure 4. State diagram model of the proposed algorithm.
SELECTING_MASTER: Each master candidate clock has a set of timestamps representing other candidates' clock values, as well as its own timestamp. Clock values of the nodes that failed to send their timestamps by the deadline are replaced with zeros. By applying the fault-tolerant midpoint function the clock which has the median value is selected as the master of the corresponding synchronisation round. On the other hand, clocks whose time differences with the median value are bigger than a threshold D will be considered as faulty.

GENERATING_SYNC_MSG_ $\alpha$ : In this state, the selected master clock sends a synchronisation message, $\mathrm{M}^{\alpha}$. The content of this message is a set of identification numbers corresponding to the substitutes, with which the faulty candidates in the MCG identified at the selection process will be replaced. An empty message represents that all the current candidate clocks are non-faulty, so that updating the MCG will not take place.

GENERATING_SYNC_MSG_ $\beta$ : The first synchronisation message $\mathrm{M}^{\beta}$ is immediately followed by another message, $\mathrm{M}^{\beta}$, which is sent by the master too. This message contains the timestamp taken by the master when the first synchronisation message was delivered to all the non-faulty clocks in the system.

SELECTING_NEW_MASTER: This state is reached when any of the deadlines for sending the synchronisation messages has expired. In this state a new master clock is selected. After replacing the clock value of the faulty master with zero, the same selection function is applied.

CLOCK_CORRECTION: All clocks in the system except the master calculate their correction terms corresponding to the differences from the master's clock value which is delivered in the follow up message $\mathrm{M}^{\beta}$. Each clock then adjusts its logical clock with the correction term to remove the clock error.

ACKNOWLEDGEMENT: If any clocks in the MCG were identified as faulty through the master selection mechanisms, it is then necessary to replace them with the clocks in the SUB group. Clocks designated in the first 
synchronisation message $\mathrm{M}^{\alpha}$ as new candidates for the master have to acknowledge their clock status by saying "accept" or "refuse". No reply automatically indicates the refuse. Each substitute clock designated as a new candidate evaluates its own status by applying a threshold $\mathrm{D}$ to the clock error calculated by the clock correction function. If a designated substitute refuses to be a candidate clock, the next clock is automatically assumed to be a candidate and has to send its acknowledgement message. UPDATING_MCG: This state is to wait until all the faulty clocks in the MCG are substituted.

MCG_READY: The $k$-th synchronisation round ends when all of the master candidates are confirmed to be non-faulty.

\subsection{Master Clock Selection}

Figure 5 shows the entire steps for the suggested algorithm. It is assumed that at most $f_{m}=1$ new faulty clock can be found in the MCG in a single synchronisation round, and thus three clocks $\left(\mathrm{C}_{1}, \mathrm{C}_{2}\right.$ and $\left.\mathrm{C}_{3}\right)$ for the MCG are needed. In this example clocks $C_{2}$ and $C_{1}$ are assumed to be the best and the faulty, respectively. Arrows represent a broadcast of message with unknown latency.

Selecting a master is performed in the first two steps of figure 5. The selection function (F) is based on the timestamps $\left(T_{1}, T_{2}, T_{3}\right)$ taken by each candidate clock on the arrival of a start message $\left(\mathrm{m}_{\text {start }}\right)$. As soon as a timestamp has been taken, each candidate, including the sender of start message, broadcasts a time message that contains its timestamp, and then waits for other candidate's time messages.

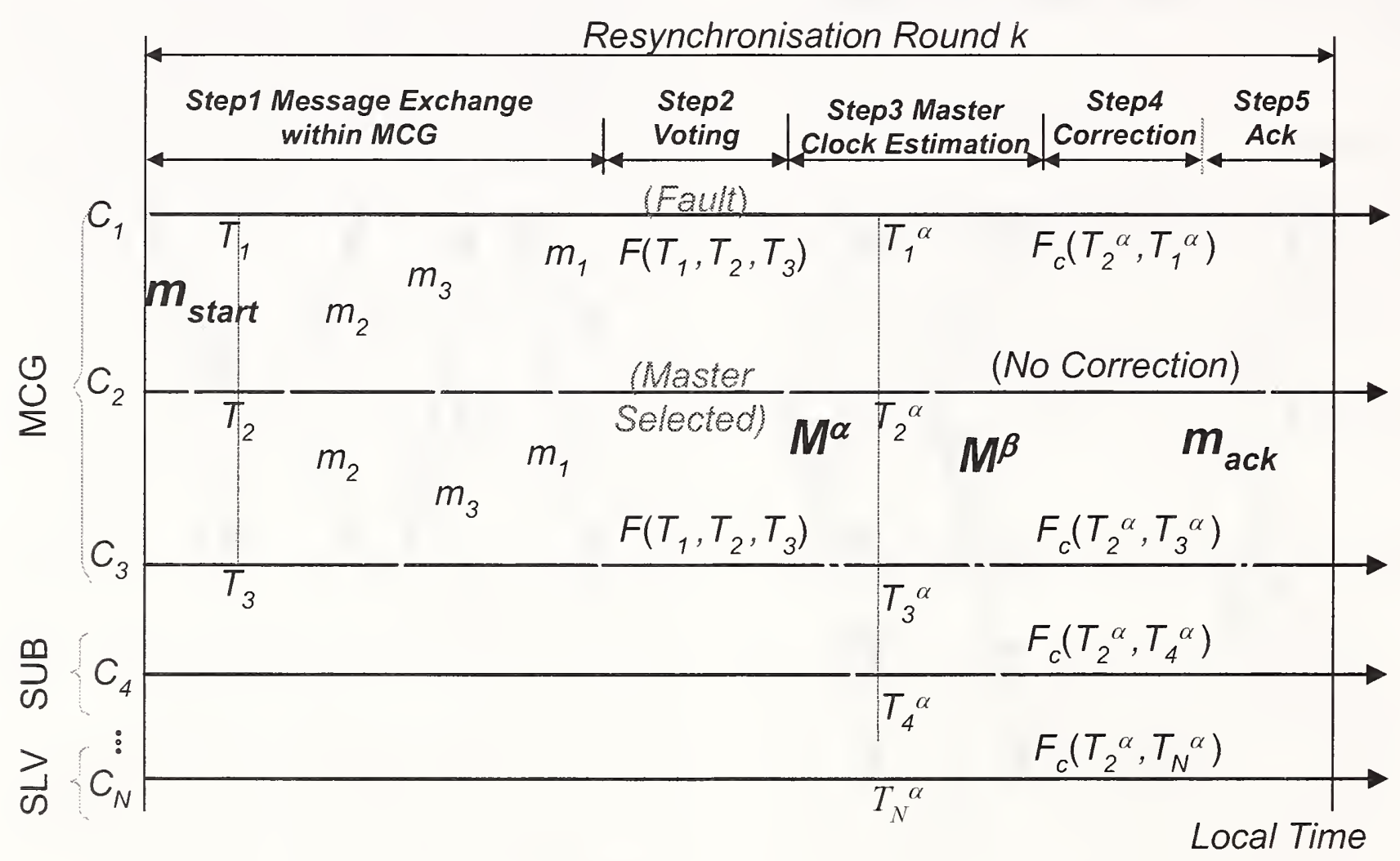

Figure 5. A timing diagram model of the synchronisation algorithm. 
Since CAN is a multicast network, all the correct candidates will obtain an identical set of timestamps, and thus, will vote for a common clock as the master.

In addition to the selection of a master, the selection mechanism also identifies any faulty clocks in the MCG. Any candidates whose time differences with the median value are larger than a predefined threshold will be considered faulty. The faulty clocks abandon themselves as candidates for a master and move to the SUB group, whilst the selected master builds a list of new candidates for the following synchronisation round.

A key advantage with the proposed voting mechanism is its robustness. Since all the timestamps needed for voting are taken at once, any delays (or even missing deadlines) in sending them do not lead to synchronisation failure. On the start messages, the fastest clock in the MCG may broadcast a "start" message. However, the selection mechanism still gives a correct result even though the resynchronisation round starts earlier due to the fastest clock (or a faulty clock in the worse case), since the selection algorithm relies on the fact that a start message has arrived rather than the time when it was generated.

\subsection{Clock Correction}

Except for the selected master, all clocks in the system synchronise to the master clock. Step3 and step 4 in figure 5 are related to the clock correction mechanism.

\subsection{Substitution of Faulty Candidates}

Each synchronisation round ends by updating the MCG with a set of non-faulty clocks (step5). The key to this process is the acknowledgement messages sent by the substitutes. Only the substitutes requested by the current master send the acknowledgement messages containing binary information, i.e., "accept" or "refuse" depending on the sender's clock status. x 4.6. Analysis
It is not feasible to describe in this paper the details of analysis on the achievable synchronisation precision and the number of messages. To summarise, the worst case synchronisation skew between any two clocks is given by:

$$
\delta=4 \rho R+\xi
$$

where, $\rho, \mathrm{R}$, and $\xi$ denote drift rate, resynchronisation period, and reading error, respectively. To tolerate $f_{m}$ faulty candidates in a single synchronisation round, the total number of messages for the synchronisation algorithm is given by:

$$
2 f_{m}+4 \leq n_{m s g} \leq(\eta+2) f_{m}+4
$$

where, $\eta=0,1,2, \ldots$ is a parameter to be selected by the system designer according to the desired degree of fault-tolerance.

\section{APPLICATION TO IEEE-1588}

The application of the proposed synchronisation method to a networked system involving any number of IEEE 1588 clocks is straightforward, because the proposed method:

- is developed for a multicast network;

- has a master-slave structure;

- does not require any assumptions by the clocks;

- use the "a posteriori" technique which can be easily replaced with the method used in the PTP (i.e., "sync" and "follow-up" messages); and

- is a software-based method;

A likely network configuration for typical embedded applications is shown in figure 6 . Some subnets may contain 1588 clocks, and others may not. 


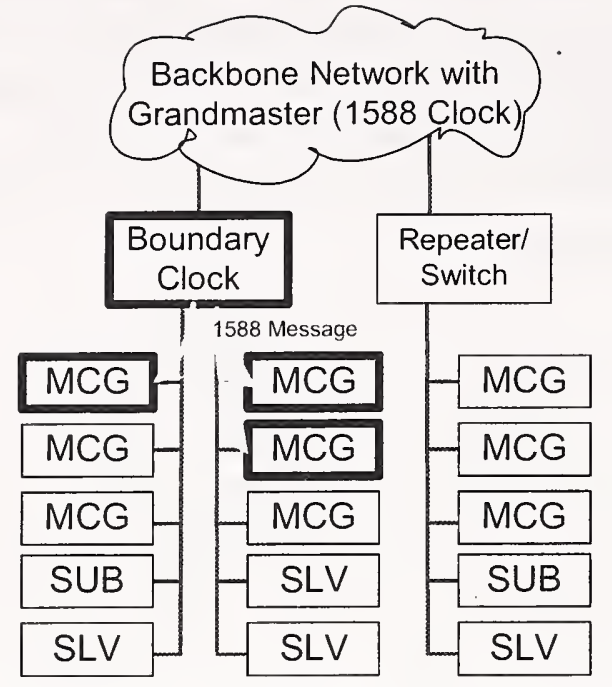

Figure 6. Synchronisation for a system with heterogeneous networks and clocks.

If the subnet contains any 1588 clocks, they can be considered as the member of MCG or SUB. By giving the 1588 clocks highest priority (i.e., the preferred master), the subnet will be synchronised to the external time sources or grandmaster as long as the 1588 clocks remain correct. In the presence of any faults with either the 1588 clocks, grandmaster clock, or the network connection, the DRTS synchronisation algorithm can still keep the subnet remain synchronised.

\section{CONCLUDING REMARKS}

This paper presented a deterministic and fault-tolerant clock synchronisation algorithm for low-cost embedded applications involving a limited number of 1588 clocks. The key advantage of this algorithm is the faulttolerance achieved by a clustering and voting mechanism that is flexible and deterministic, but simple. The proposed method can easily be implemented within subnets involving any number of clocks with various accuracy and types including 1588 clocks.

\section{REFERENCES}

[1] IEEE-1588 (2002). "Standard for a precision clock synchronization protocol for networked measurement and control systems", November 2002.

[2] Gergeleit, M. \& Streich, H. "Implementing a distributed highresolution real-time clock using the CAN bus", Proc. $I^{\text {st }}$ iCC, 1994. 


\section{PTP in switched networks}

\section{Topics}

- Switch architectural issues

- Discussion of sources of synchronisation errors

- Adjustment of time in an PTP client

- Discussion of required update frequencies

- Influence of cascading

- Proposed enhancements

Switch architectural issues

- Switches forward Ethernet frames based on address tables learned on source addresses while forwarding

- Switches have storage capabilities to buffer frames in case of a congestion

- The delay of switches is not fixed but will change with

- The load of the switch on a particular send port

- The time to find a destination address in the address table

- Other delays due to switch internal issues may occur

- Even in case of highest priority frames a delay of a full size frame (approx. $125 \mu \mathrm{s}$ at $100 \mathrm{Mb} / \mathrm{s}$ ) can occur (switching according to IEEE 802.1D assumed - preemptive strategies can improve this but has some other side effects) 


\section{Switch effects to time synchronization}

- Switches will produce asymmetric delays as frames will delay time messages

Additional Delays at Delay measurement

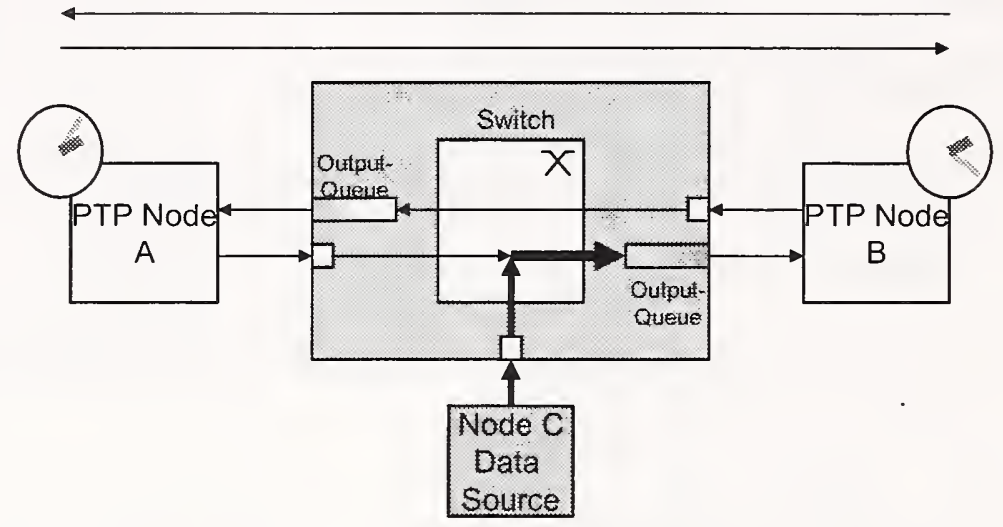

- Trying to filter delayed frames will lead to unpredictable results

\section{Discussion of sources of synchronisation errors}

- 3 types can be identified

- Jitter: random change of clock signals

- Delay: time offset between two clocks

- Drift: deviation due to frequency shifts of local clocks

- Jitters can be adopted by filtering (if it is of statistic nature)

- Delays are caused by non symmetrical paths between clock receiver and clock sender (this are a few ns at physical level but may be $100 \mu$ s or more in case of non-preemptive switches)

- Drifts can be measured at start up as a difference of the difference of a pair of local time and received time

- Drift will change with aging (very slow) and temperature change (moderate with a deviation of 1 PPM per degree of Kelvin) 


\section{Drift compensation issues}

- Drifts may occur at a cyclic base

- The cycle time is not correlated to the drift change

- Filtering will not be adequate as frequency is not fixed

- A control loop with PI should be used to solve this issue (Cycle $=$ Cycle of control loop)

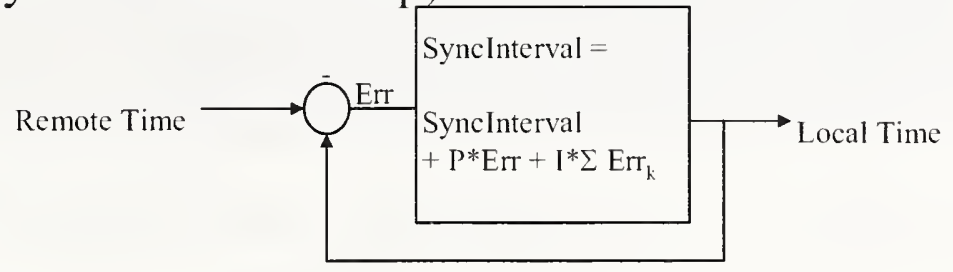

- The following parameter set will produce acceptable results

- $\mathrm{P} \quad 0,75$

- I 0,25

\section{Example(1): Synchronisation of a pair of nodes}

- Error-Phase of Sender is reverse to Error-Phase of Receiver

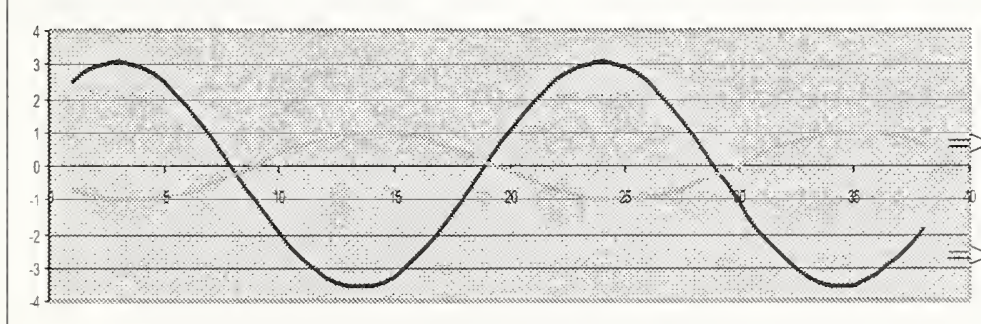

Error of Clock Receiver (relative Value)

Error of Clock Sender (absolute Value)

- Error Frequency of approx 20 cycles, Error of 1 unit $\Rightarrow$ even in that case the Error is about 1,2

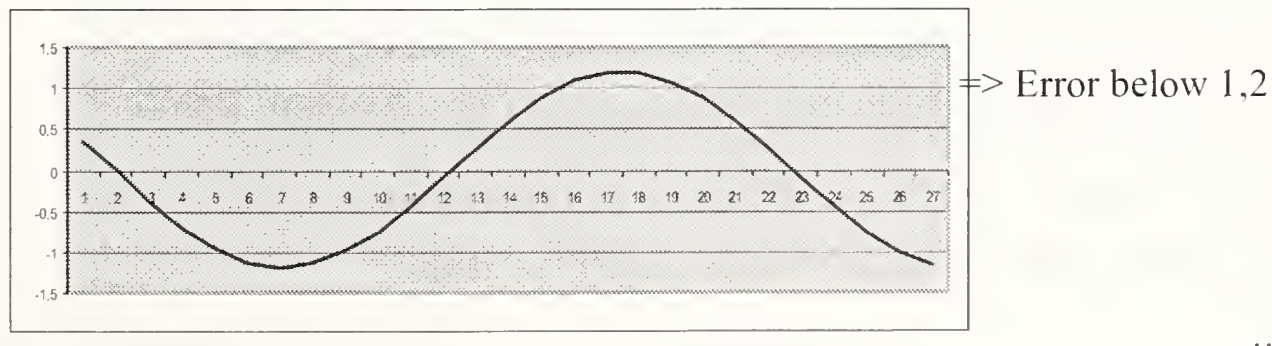

$9 / 29 / 2003 \quad 6$ 


\section{Example(2): Synchronisation of a pair of nodes}

- Error Frequency of 10 SyncIntervals, Error of $+/-1$ unit (at sender and receiver)

$=>$ results in an error of approx. 1,6 units

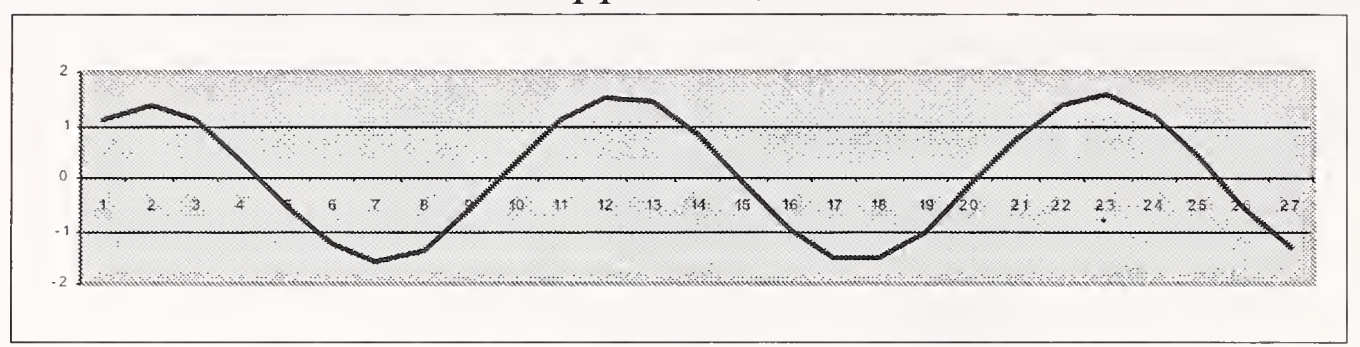

- Error Frequency of 2 SyncIntervals, Error of 1 unit $=>$ results in an error of approx. 1,8 units

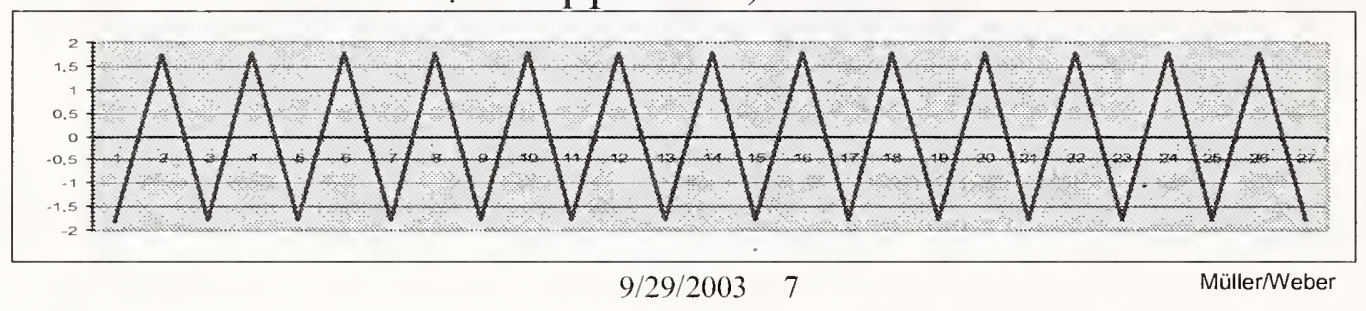

\section{Discussion: update frequencies}

- In case of a control loop cycle of $1 \mathrm{~s}$ : a cyclic temperature change of 1 degree within $10 \mathrm{~s}$ $\Rightarrow>$ results in an error of approx. 1,2 Microseconds .....see Diagram of Example(1)

- IEC 60068-2-14 requires temperature changes of 1 degree $\mathrm{K}$ in 20 s and this is not the only cause of error

- According to Example(2) a higher Error may occur in case of higher frequencies

- Errors due to jitter (or filters) may be added

- Consider to use shorter clock synchronization cycles

- $1 / 2,1 / 4,1 / 8,1 / 16,1 / 32$ seconds shall be used 


\section{Influence of cascading}

- As switches have an unacceptable jitter there should be a something like a border clock in every switch

- The number of switches between two nodes determines the degree of cascading of clock control loops

- Example: 3 Switches between Source and Destination

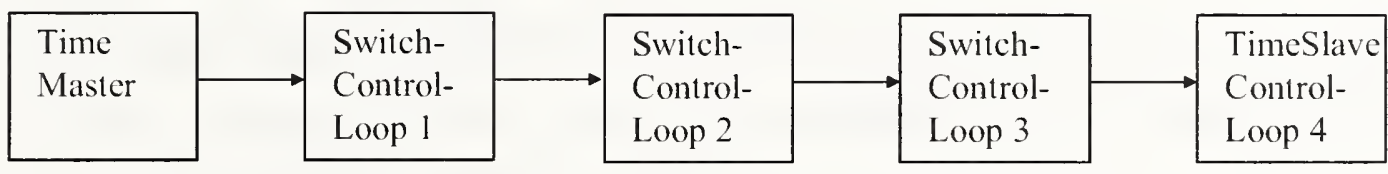

\section{Discussion: Cascading}

- Cascading is a risk in control loop design

- In small configurations, the system may work

- The larger the system the higher the risk of instability

- The problem is, that errors will be accumulated

- But the control loop cannot follow an error signal quickly

- A restricted set of control loop parameter may help but at the end the system will not be able to follow a drift change. 


\section{Cascading Example(1) : 3 Switches in a row}

- All 5 clocks have a drift change to +/-1

(frequency of 10 SyncIntervals)

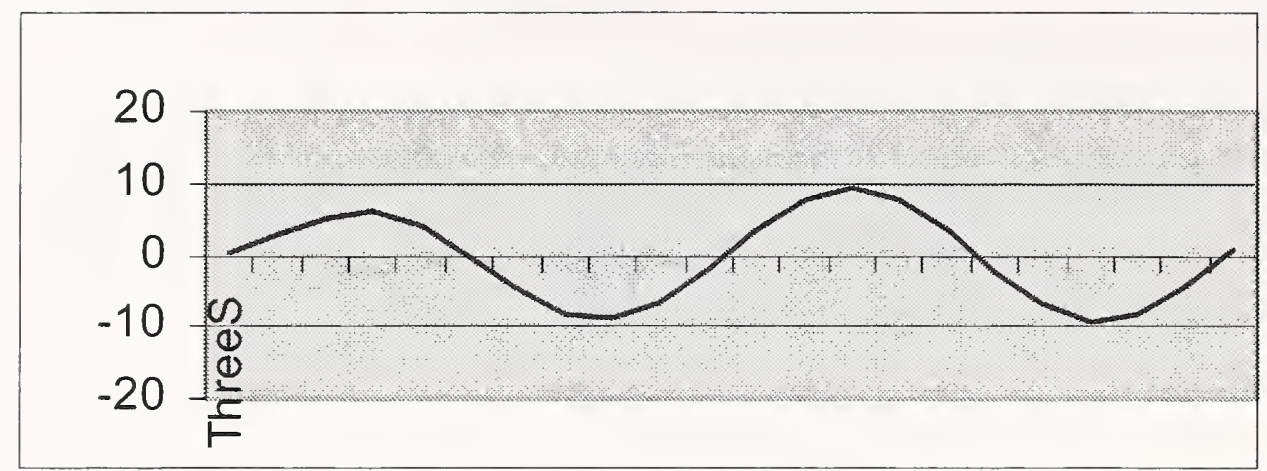

- The short Term drift is more than 8 times higher as the drift of a master-slave configuration

\section{Cascading(2): with more Switches}

- Same Situation witch 5 Switches

$\Rightarrow$ error must be below 0.1 PPM in $10 \mathrm{~s}$

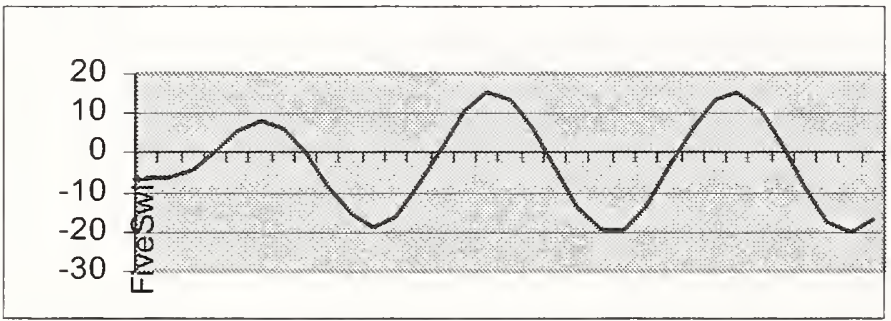

- Same Situation witch 10 Switches

$\Rightarrow$ no chance to handle this case with an error of $1 \mu \mathrm{s}$

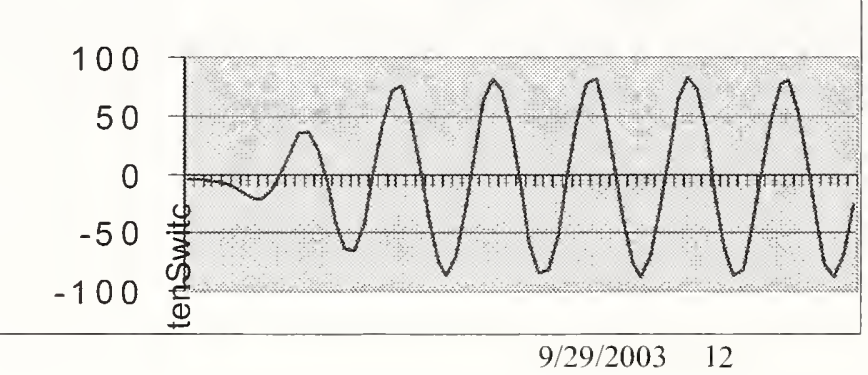




\section{Proposed enhancement in PTP}

- Introduce a new type of intermediate clock

$$
\text { bypass clock }
$$

- The Delay within a switch will be forwarded as extra parameter

- Only nodes requiring time will run a control loop

- Switches may maintain a clock but its local time is not used in the time forwarding process

- No additional hardware requirement ("just protocol")

- The design of the control loop in that way is much simpler and more robust

\section{Principles of operation}

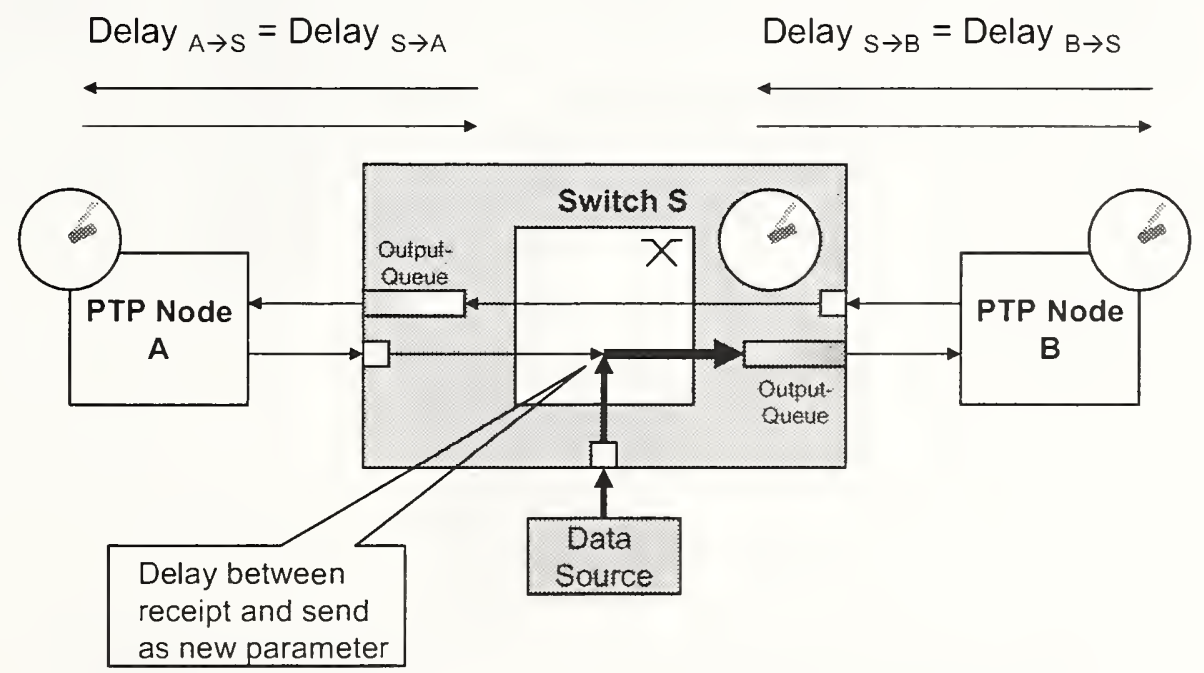




\section{Protocol considerations}

- The protocol is close to link layer and shall be treated as link layer protocol

- This protocol is should be restricted to a single link between switches and between switch and his adjacent DTE

- IEEE 802.1D reserved addresses that may help to resolve compatibility problems with non bypass switches:

Frames containing any of the group MAC Addresses specified in Table 7-9 in their destination address field shall not be relayed by the Bridge. They shall be configured in the Permanent Database. Management shall not provide the capability to modify or remove these entries from the Permanent or the Filtering Databases. These group MAC Addresses are reserved for assignment to standard protocols, according to the criteria for such assignments (Clause 5.5 of ISO/IEC TR 11802-2). 


\section{Impact of Switch Cascading on Time Accuracy \\ By Prof. Thomas Müller and Karl Weber}

Switches are the bases for modern Ethernet technology. Switches allow running networks with several hundreds of nodes without configuration overhead and little delays. Hubs limit the number of nodes for reasonable performance and routers add additional delay and limit the protocols being used.

To use switches needs special attention for Time Synchronization, because the general paradigm of symmetrical delay does not hold. Figure 1 shows the impact of other ports on forwarding PTP frames. Node $\mathrm{C}$ will add delay by filling the output queue to PTP Node B. Bridges are nonpreemptive according to the Standard IEEE 802.1 D. If Node A is PTP Master the additional delay on a delay response message to Node B will result in an error (Node B assumes a higher delay and the local time will be ahead).

There are some ways to reduce the effect of delays; one is priority tagging according to IEEE 802.1Q(give PTP frames higher priority than other frames). But this can produce a delay of $122 \mu \mathrm{s}$ in case of $100 \mathrm{Mb} / \mathrm{s}$ Ethernet for frames with highest priority per switch. The cascading of switches depends upon the application. Office applications will minimize the number of switches by using stackable switches with dozens of ports. Industrial environment need systems with flexible configurations and small switches to fit into a small cabinets. Wiring follows cabling channels that lead to a structure of a line with some trunks. Thus, an industrial control system may have tens or even up to hundreds of switches cascaded.

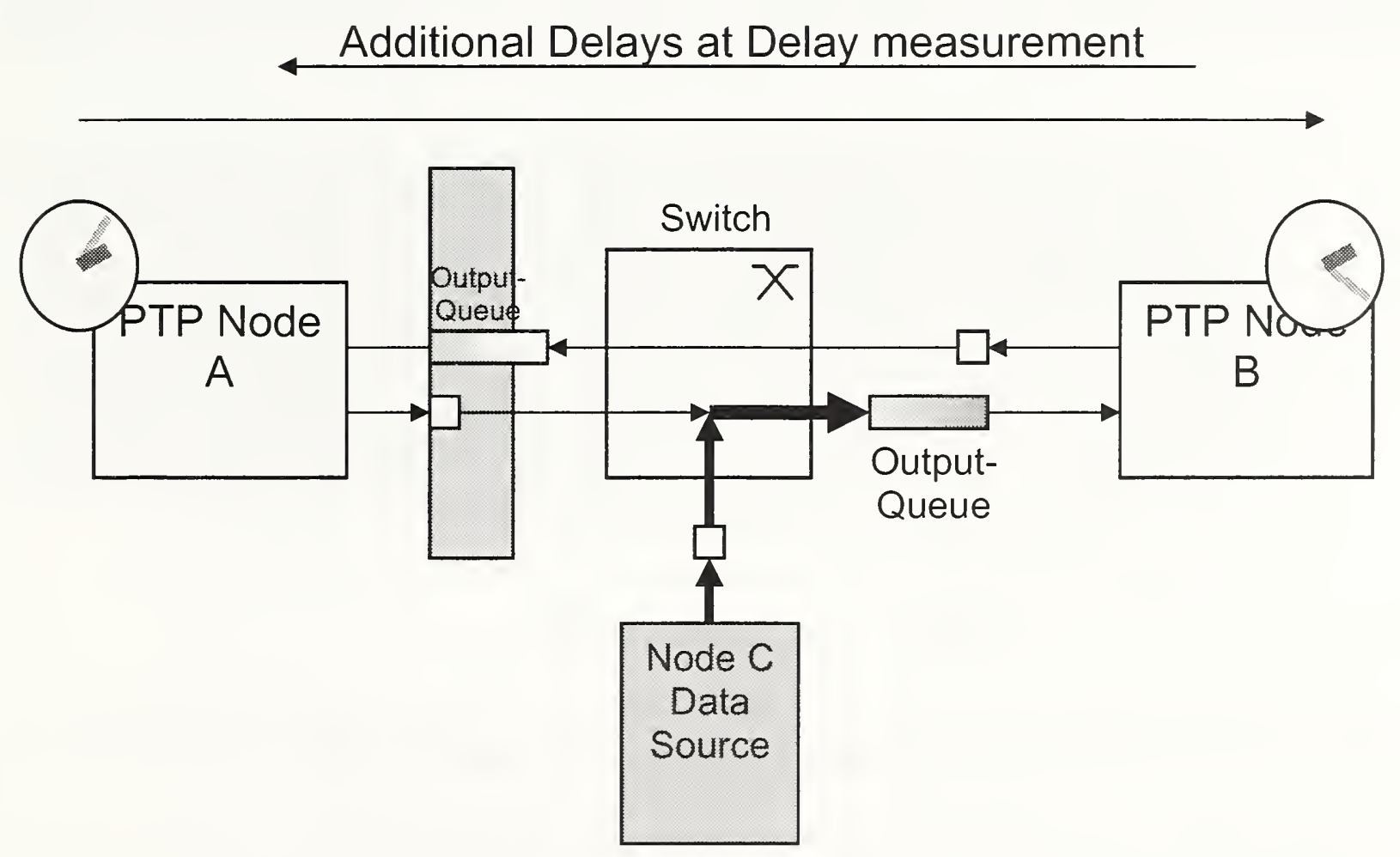

Figure 1: delay measurement with delay in one path

As local delays are not fixed, switches have to be somewhat like a boundary clock. But this requires a time adjustment in any switch.

The sources of time errors have to be determined to discuss this adjustment. Three types can be identified:

-Jitter: random change of clock signals 
-Delay: time offset between two clocks

-Drift: deviation due to frequency shifts of clocks

Jitter can be compensated by filtering (but this will additional delays to the control loop).

Delays at the transmission channels are fixed and several hundreds of nanoseconds for maximum extension of a point-to-point network. They can be compensated if they are symmetrical. As Transceiver/Receiver may not be symmetrical a correction value has to be added. The same applies for non-symmetrical cables (allowed delay skew $50 \mathrm{~ns}$ for $100 \mathrm{~m}$ according to ISO/IEC 11801). A value of 30-50 ns should be a base to estimate delays between two adjacent nodes. This is an absolute error, which cannot be compensated.

Drifts are mainly caused by deviation of the frequencies of the clock sender from the frequency of the clock receiver. They are mainly caused by temperature and ageing. As temperature change may occur in various kinds they should be compensated by a control loop. Each PTP slave has to have such kind of loop. The loop design is beyond the scope of PTP. But their design determines the quality of time synchronization and should be fixed for boundary clocks. It is of less importance for end nodes, which have no networking purpose.

Figure 2 shows the outline of a control loop. A PI-Loop is the most popular and robust design method for control loops with unknown behavior. The I-Factor should be about 1 Quarter of the PFactor and the P-Factor should be less than 1 to avoid instable conditions.

As control loops should be able to be cascaded, a P of 1 and an I of 0.25 seemed to be appropriate. This parameter are an example to show the effects of cascading.

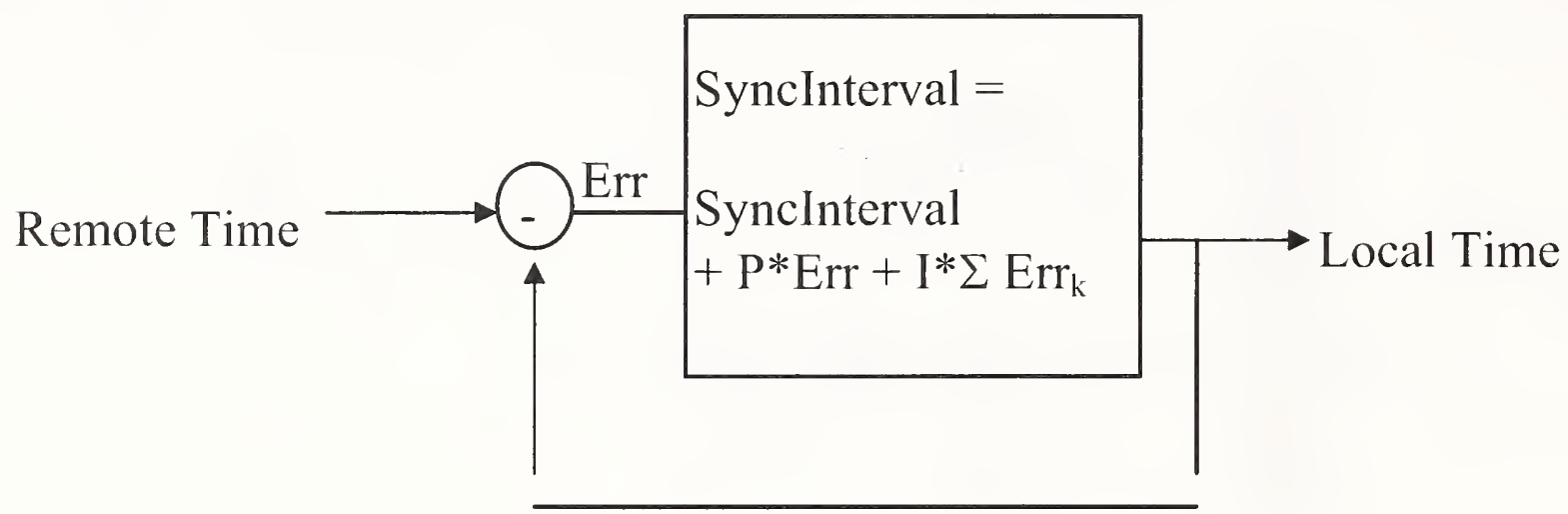

Figure 2: control loop design

Time errors are accumulated i.e. a relative time error of one unit will result in an absolute error of one in the first cycle, two in the second cycle, three in the third cycle and so on.

Note 1 the calculation was done with relative values which can be obtained by multiplying it with a time value.

Note 2 the absolute cycle time is of no importance - the maximum change and the value of an error signal have a correlation to the worst case diagram.

Figure 3 presents the basic configuration, with 2 nodes. The blue function represents the error of the PTP Master in correlation to absolute time. The purple function is the relative error of a PTP Slave. This diagram shows error changes as cyclic function which may occur in areas with regulated temperature. Other waveforms are tested with similar results. 
Figure 3 shows an absolute error of about 3 if the error cycle is about 20 Sync Intervals. The absolute error is of less importance but it will lead to time deviations. The numbers are selected because a temperature change of 1 Degree Kelvin will occur within 20s in the IEC 60068-2-14. So this figure represents a time synchronisation interval of $4 \mathrm{~s}$ without statistical filters and about $1 \mathrm{~s}$ with filters averaging the last 8 values.

tests. The selection of this parameter does not affect the general result. A longer cycle will reduce the absolute error at the same amplitude if the error slope is in the same range.

Figure 4 and 5 shows results for a error cycle of 20 Sync Intervals and 10 Sync Intervals with the same error maximum value. The resulting error in the Figure 5 is 1.8 times which is caused by the higher slope.

Figure 6 show the result of an error cycle of 40 Sync Intervals which is smaller because of the reduced slope.

It might be difficult to have a sub microsecond accuracy with standard clocks even in the case of no boundary clocks between because the error due to temperature drift is even higher as the requested mikrosecond.

In case of a Sync Interval of $4 \mathrm{~s}$ (or filtered with a Sync Interval of 1s) and cyclic temperature change of 1 degree within 20s will results in an error of approximately 1.7 Microseconds.

A shorter cycle time will improve the situation. The negative powers of 2 may be used without changing protocol formats.

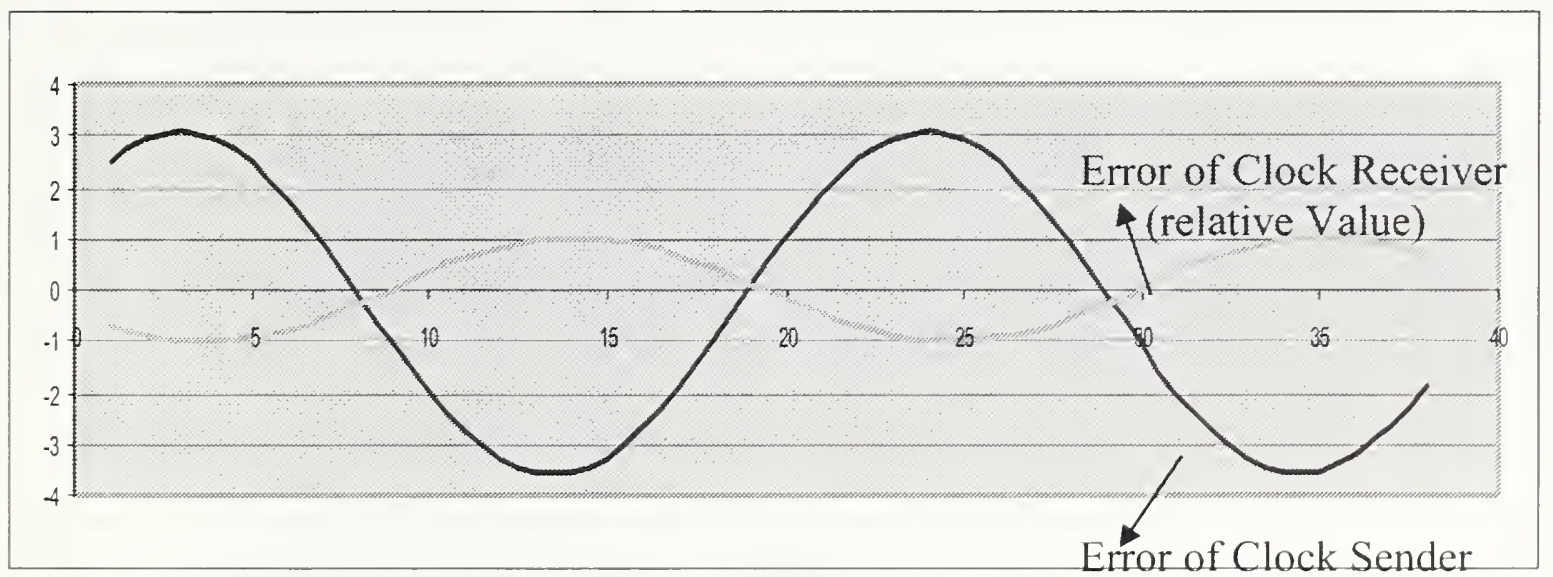

(absolute Value)

Figure 3: Absolute error and relative error at receiver 


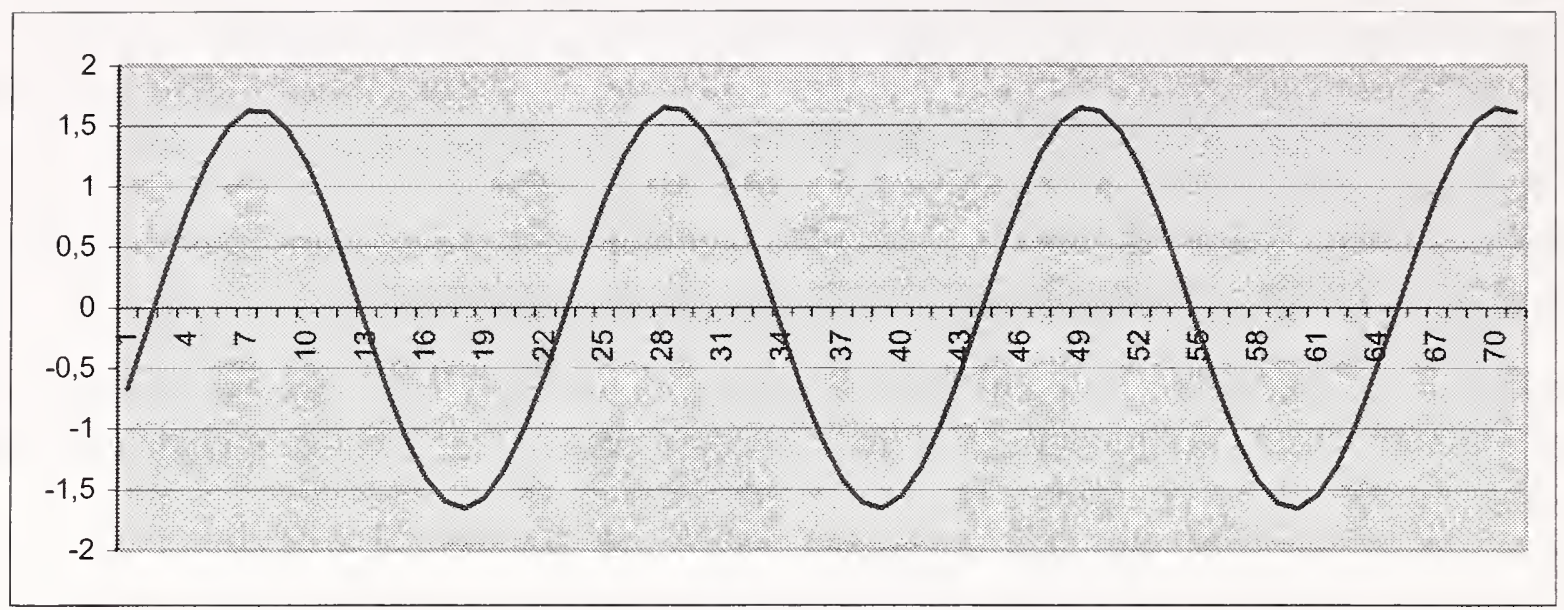

Figure 4: Error between sender and receiver(Error cycle $=20$ SyncIntervals)

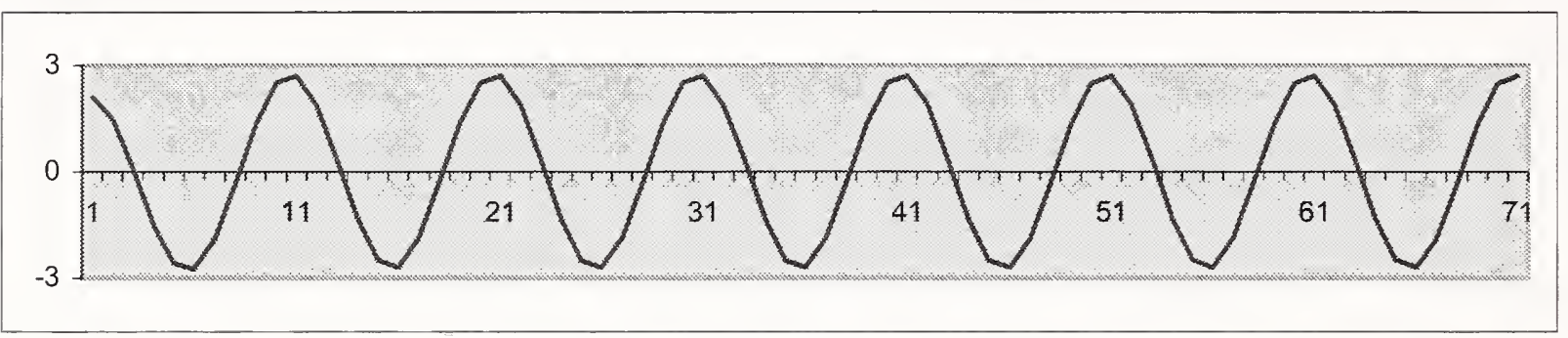

Figure 5: Error between sender and receiver(Error cycle $=10$ SyncIntervals)

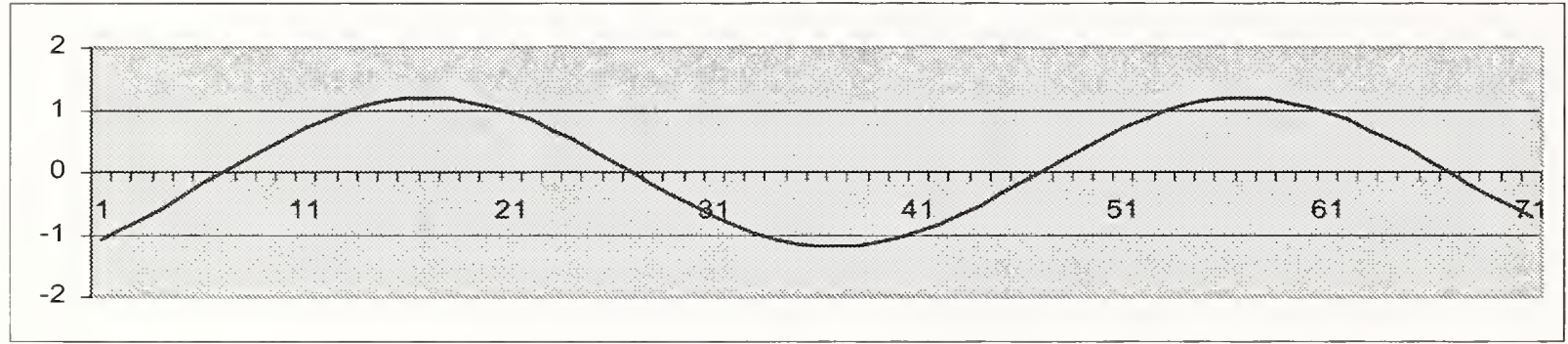

Figure 6: Error between sender and receiver(Error cycle $=40$ SyncIntervals)

As switches have an unacceptable jitter there should be a something like a border clock in every switch. The number of switches between two nodes determines the degree of cascading of clock control loops. If there are 5 Switches between PTP master and PTP slave the number of cascaded control loops is 6 .Figures 7,8 and 9 shows that the growth of the error is non linear. Therefore the cascading may work for configurations of 3 to 5 Switches but may be completely inacceptable when the number of switches is 10 or even higher. Even in case of small drifts in the area of less than 1 PPM over 20 Synch Intervals the result would be an error in the 50 microseconds range. 


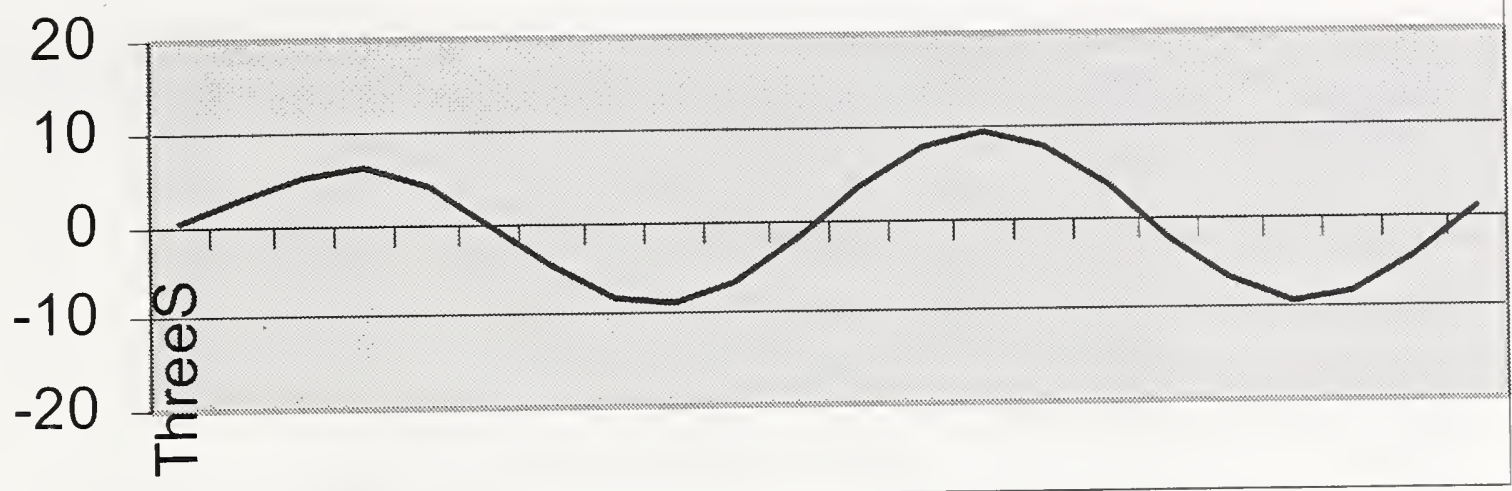

Figure 6: Error between sender and receiver(with 3 Switches in between)

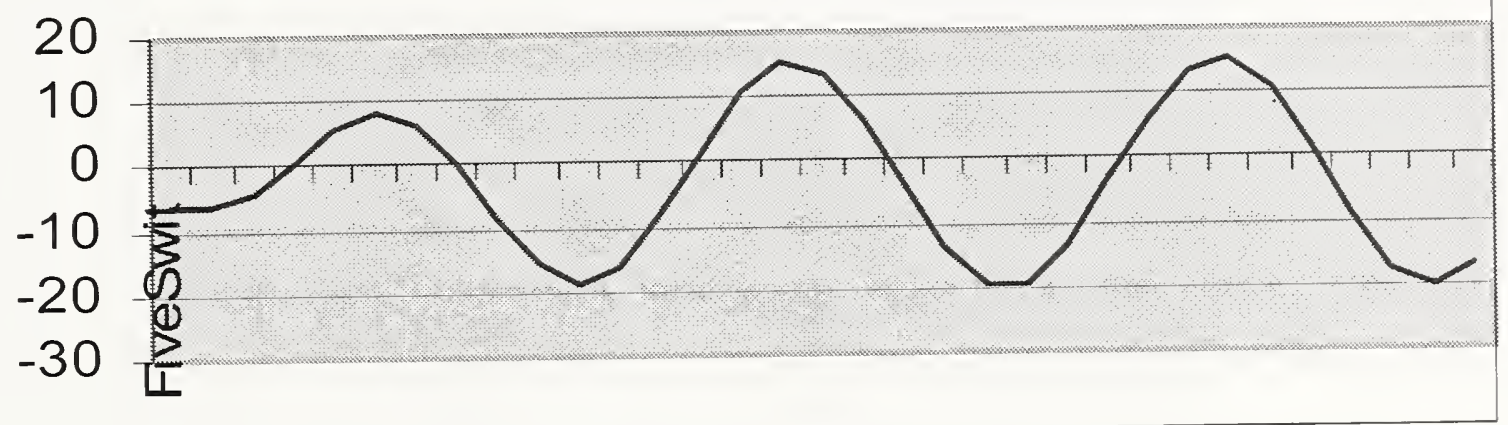

Figure 7: Error between sender and receiver(with 5 Switches in between)

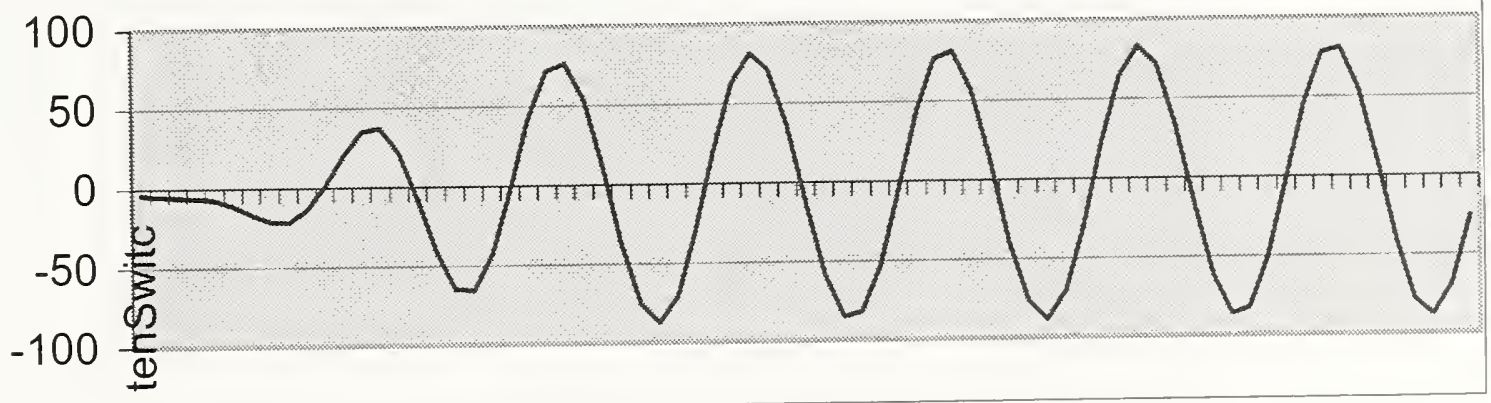

Figure 7: Error between sender and receiver(with 10 Switches in between)

The main cause of the problem is the control loop in the switches. The need to do this is because of the dynamic internal delay of the switches. PTP itself should have all means to measure this internal delay (= time outgoing - time incoming of the sync frame). The problem could be solved by in a system that sends the delays in the frames. If switches will do this a very simple solution of this 
problem can be found. Control loop design for switches may be much simpler. There is additional jitter due to cascading, but as it is of statistical nature it can be managed by filters easily. Switches with no need for synchronized time may not run a control loop.

There are a few additional thoughts how to handle PTP in a protocol architecture:

- The protocol is close to link layer and shall be treated as link layer protocol

- This protocol is should be restricted to a single link between switches and between switch and his adjacent DTE

- IEEE 802.1D reserved addresses that may help to resolve compatibility problems with non bypass switches: 


\section{IEEE 1588 and Network Devices}

Hirschmann Electronics GmbH \& Co. KG Automation and Network Solutions

Dirk S. Mohl

Part 1: IEEE 1588 - SW Implementation and Design Results

- System Architecture

- Software Architecture

- Protocol Simulation

- Ideas for Improvements of Precision of Software Stack

- Software for Linux, Windows and VxWorks 


\section{System Architecture of IEEE 1588 Implementation}

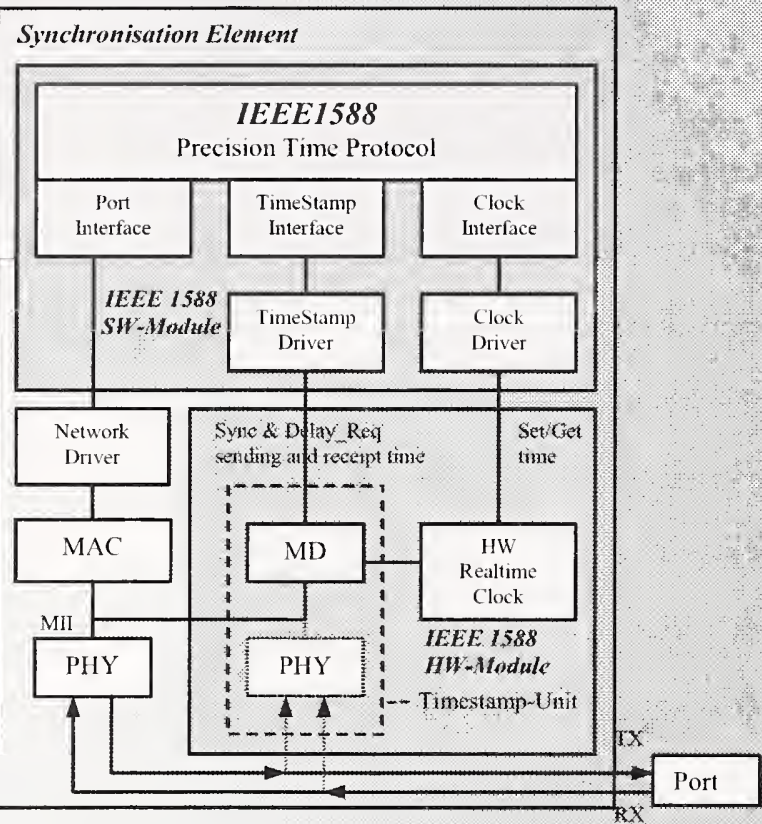

........... necessary if no MII- interface available

MD - Message Detector for Sync and Delay_Request packets

Main Goal: Operating System Independent Design

- OS independent Protocol Stack (1EEE1588 Implementation)

- OS Absuracion Laver

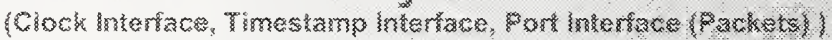

- OS dependent

(Tasks, Thmer, Semankors, Sockers)

- OS and Hardware dependent

(Network Driver, Clock Driver, Trmestamp Drisge? 


\section{Basic Software Architecture}

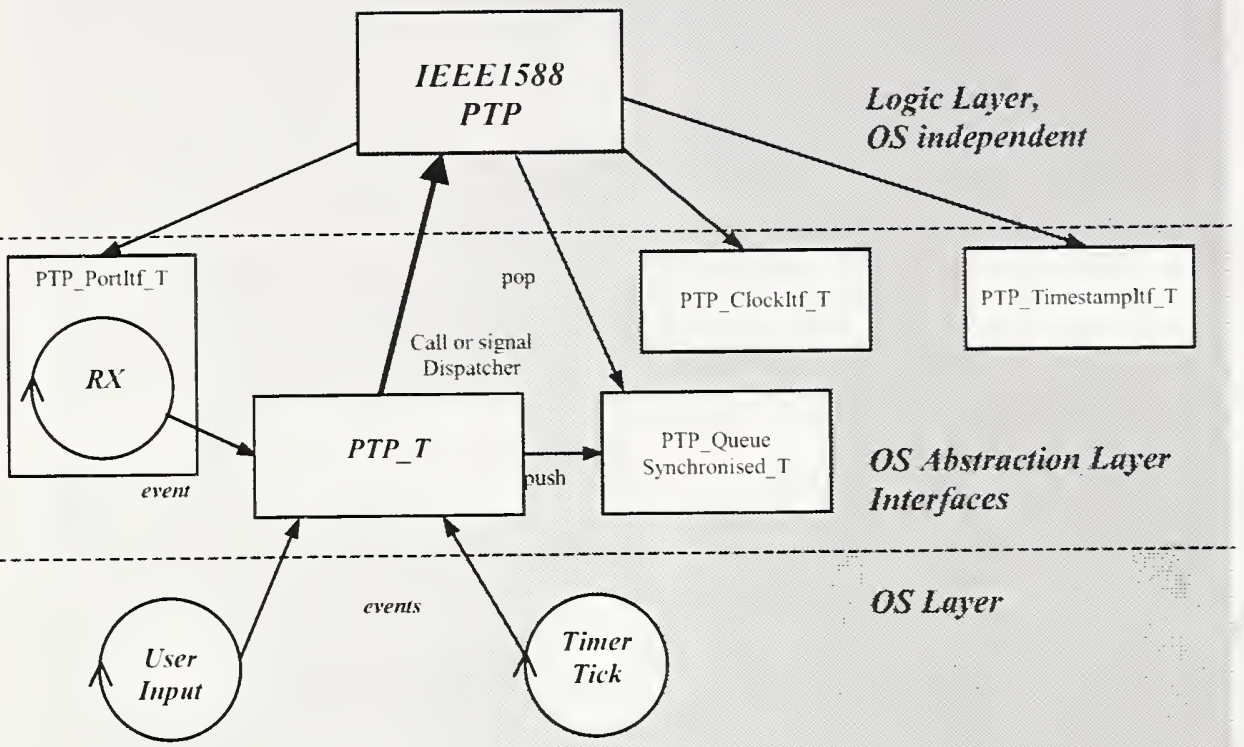

\section{Linux Software Architecture}

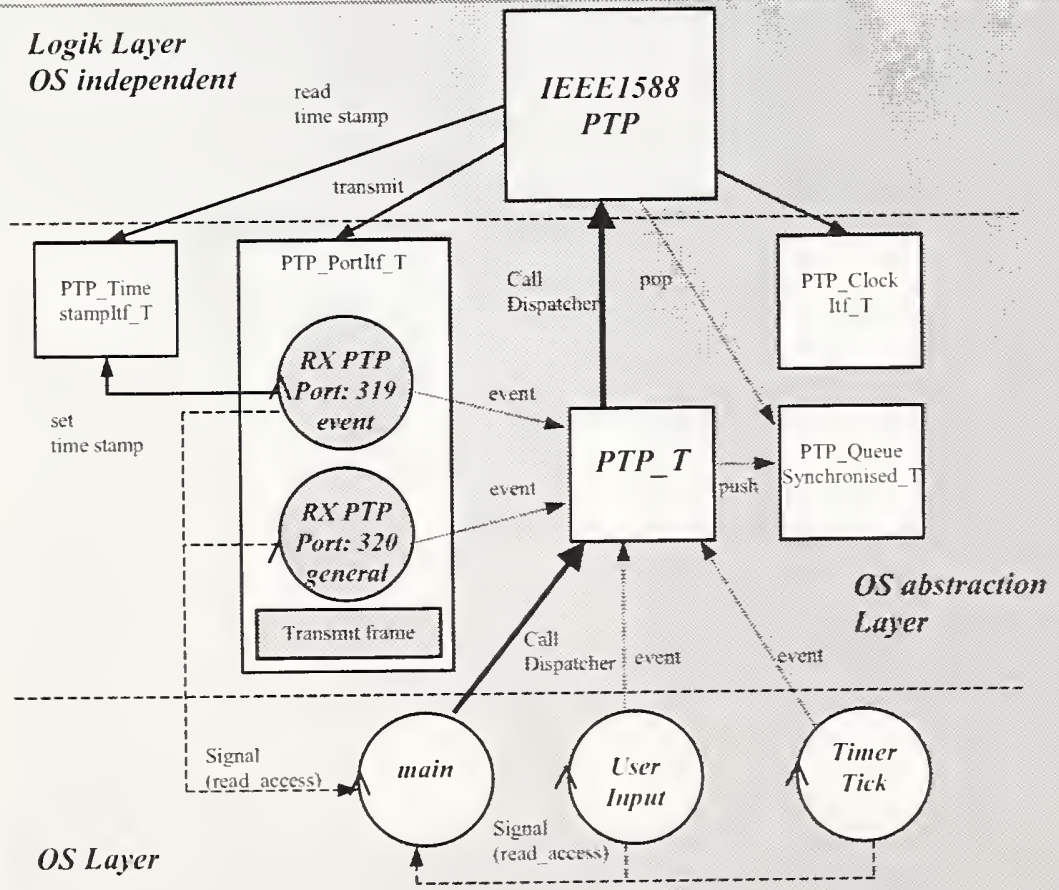




\section{IEEE 1588 Protocol Software Architecture (Ordinary Clock)}
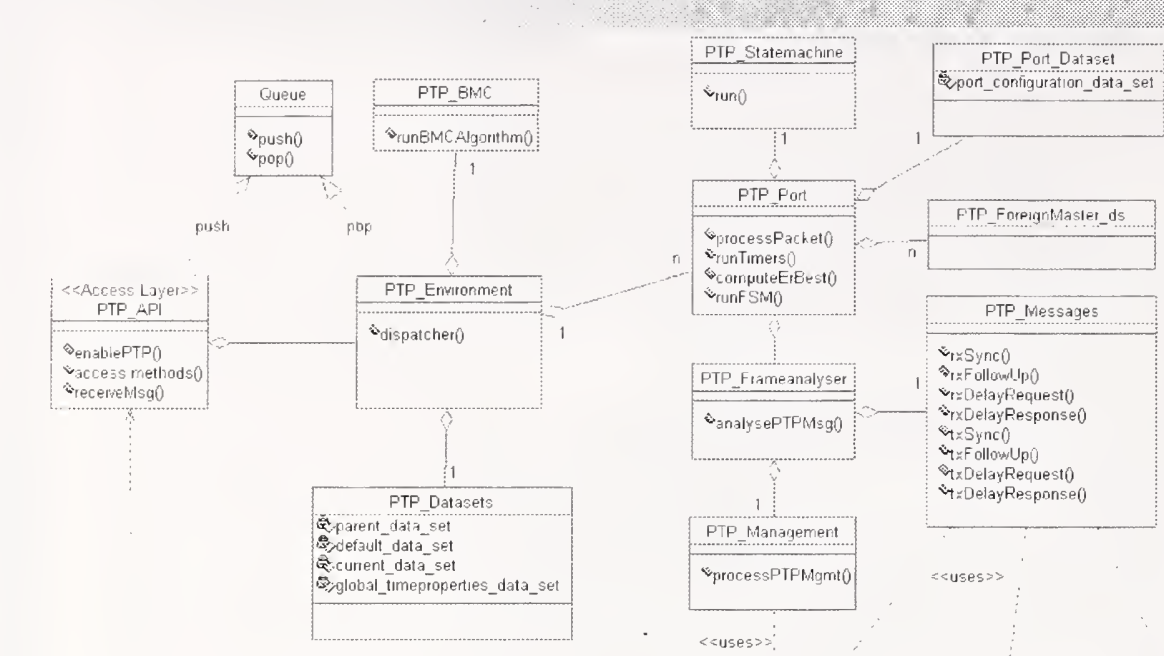

recerve

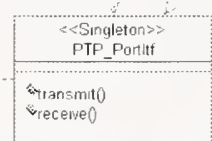
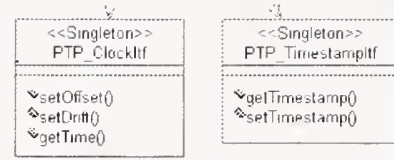

IEEE 1588 Protocol Simulation

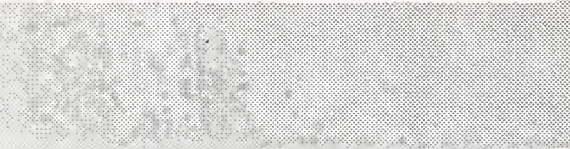

\section{(EEm}

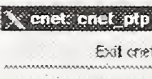

Exit (ret

Me ssagyes delivered carrectly:

zavee topoobory

Stengle grent

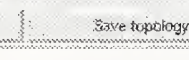

axtoos)

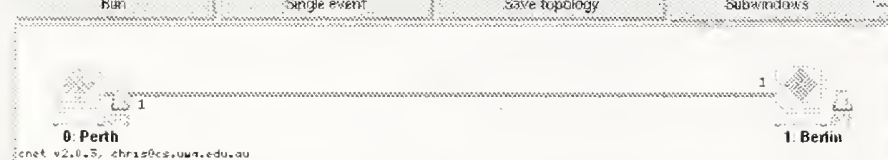

Q. Perth

1. Beniin

(3)

1588 Implementation in $\mathrm{C}$ Code

Running in the network simulator "onet"

Protocol Verfication for

- Point-10-Point Trafic

- Boundary Clocks

- Best Master Clock Algorthm

- Management Protocol

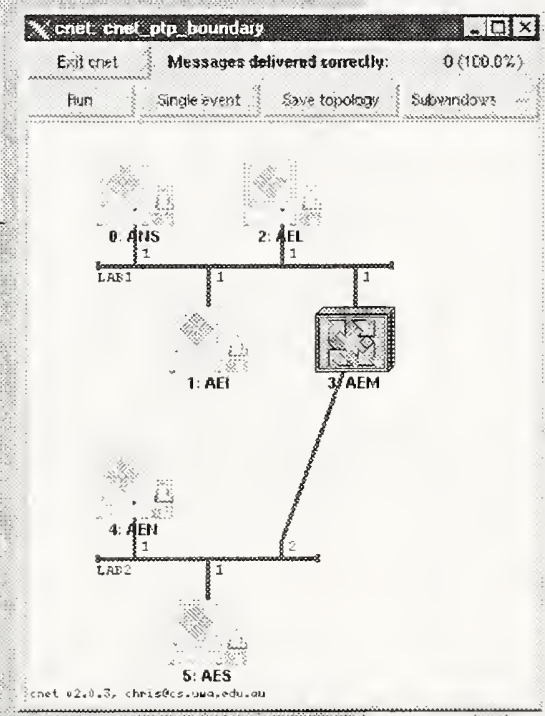

Network simulator cnet is available at httn:/ www.cs.uwa.edta.aucnet 
Improvements of Software Stack

First Step

- Using UDP sockets / Winsock

- High task priority for PTP

- Manual drift / rate compensation for high precision counter

$\Rightarrow$ Jitter typical $+/-10 . .100$ us

\section{Second Step}

- Algorithm for throwing away sync packets which are too late

- same for delay request packets

- Automatic drift / rate compensation

$\Rightarrow$ Jitter typical $+/-5 \mu \mathrm{s}$

\section{Third Step}

- optimized ISR: Software Timestamp for TX and RX packets: basics research done by ZHW

$\Rightarrow$ Jitter expected $+/-1 \mu s$

Assumption: no high Network traffic, no high CPU load (e. g. no Bus Master DMA

\section{Software Library Concept}

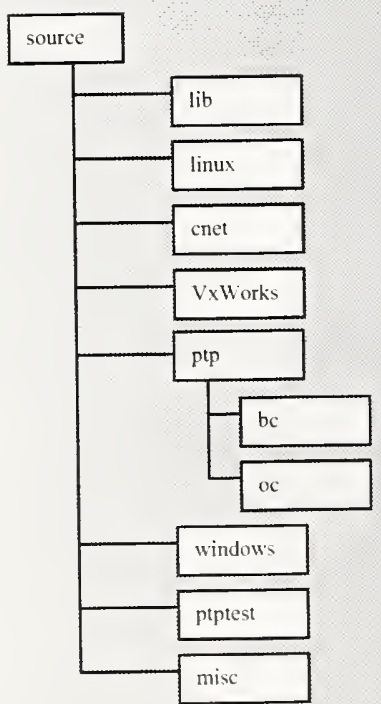

Hararies

Linux Interfice Into

Cnet Simulation

Vxworks Huterface inapl.

OS indeperutent

Boundiry Clock specifac

Ordirary Clock spexific:

Windows Interface fmpt.

PTP Test Program

Hodules for wirkterws and tanux

- Linux Boundary Clock: Code from directortes ptp, bc, misc und Hnux

- Linux Ordinary Clock: Code from directories ptp oc misc und finux.

- Linux Ordinary Clock: Code from directories ptp, oc. $p t p, b c$, misc und windows

- Windows Boundary Clock: Code from directones pip, bc, misc und windows 


\section{IEEE 1588 Linux and Windows Implementation}

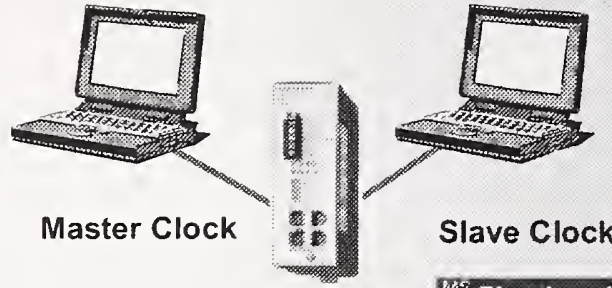

offer

Ofrse: correction by arit / rate control

System Clock of Windows (approx. Ius)

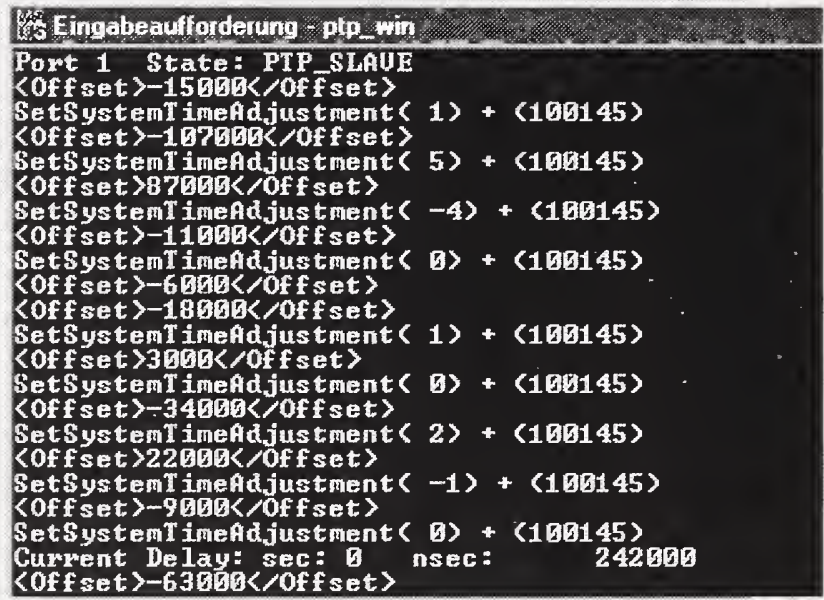

\section{Summary of Software Stack}

- FUIIEEE $1588-200 \%$ mplementation

- synchronization, follow up, delay measurement

- best master clock algorthm

- TUII IEEE 1588 management support

- addutunal features

- drif/rate correction

- jiter fher for synchonization and dalay measurements

- porable code

- adaptation layer for pure sottware or hardware based time stamping / clock generation

- SNMP MIB (VXWorks)

- time representation of nsec: UINT32

- Masted under Linu, VxWorks and Whdows

- Precision typically in the range of 10us (sW time stamp) 
Part II: IEEE 1588 - Layer 2 Switches as Boundary Clock

- Why IEEE 1588 Switches with Boundary Clock

- Boundary Clock Software and Hardware Architecture

- Prototype: Modular IEEE1588 Switch

- Results of Hardware implementation

IEEE 1588 and Switches whthout Boundary Clock

Latency of Layer 2 Switch - store and forward

Packet reception depending on packet length:

100 Mbits/s: $5,8 \mu$ s for 64 Bytes up to 122us for 1518 Bytes

"last bit in first bit out" latency of a Switch:

typical hardware based, no cascaded chip: 2 .. 5 us

Measured Jitter of switch latency:

e.g. Hirschmann Switch RS2-FXJF: 0,4 us

But this values are only valid under "no load" condition 


\section{IEEE 1588 and Switches without Boundary Clock}

The Problem:

Jitter under load condition:

Not depending of the Hardware, but depending on queued packets $=T \times$ queue length of swich worst case for e.g. a 20 packet queue: Jiter up to $20 \times 120 u s=2,4 \mathrm{~ms}$

Does prioritization help?

Alleast one low priorily packet can still be in process of transmission $=>$ jitter up to 125 us (maximum length packet) bul current available swiches show that atter the prionity scheduler there is another queue for 2 up 108 packets $=>$ inter $360 \mathrm{~ns}$ up to $1 \mathrm{~ms}$

$\Rightarrow$ avalable priontization e.g.801.D/p does not really help

\section{IEEE 1588 Switch: Boundary Clock on Layer 2}

The solution:

Use IEEE 1588 Boundary clocks in switches

- only point to point comections:

$\Rightarrow$ nearly no delay jitter between master and slave

- internal queuing delay/jitter of swich not relevant

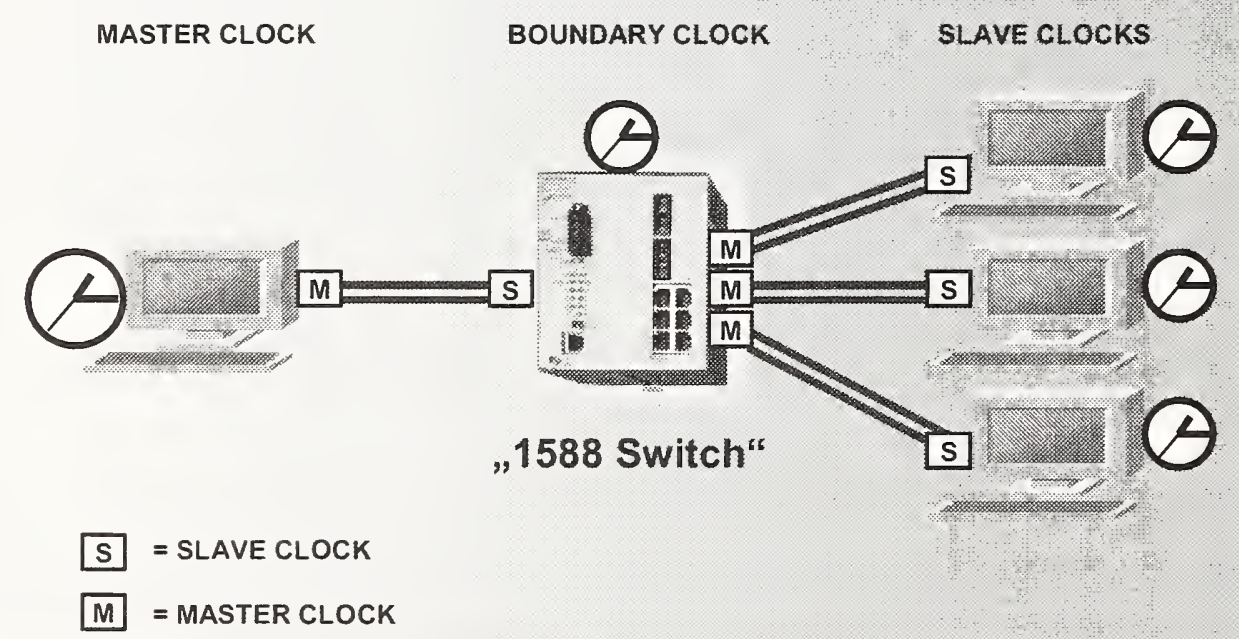


IEEE 1588 Hardware Architecture - Message Detector

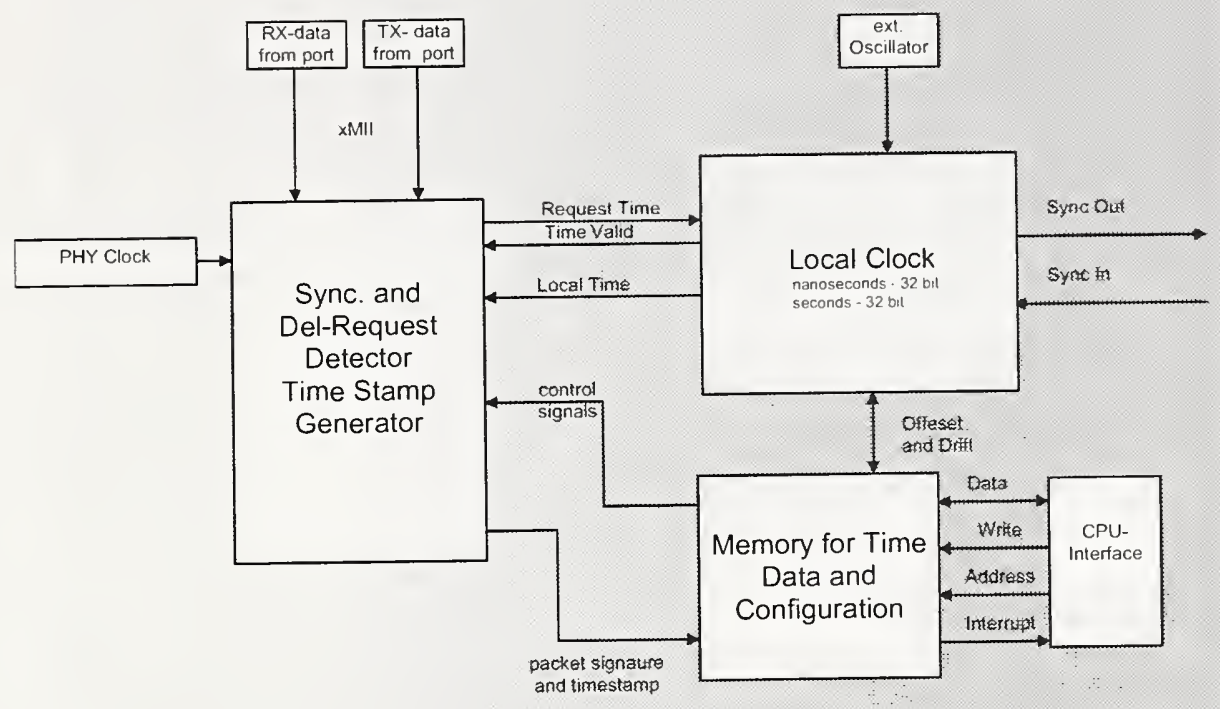

Design of Sync Message Detector and Clock

IEEE 1588 Modular Switch: Prototype
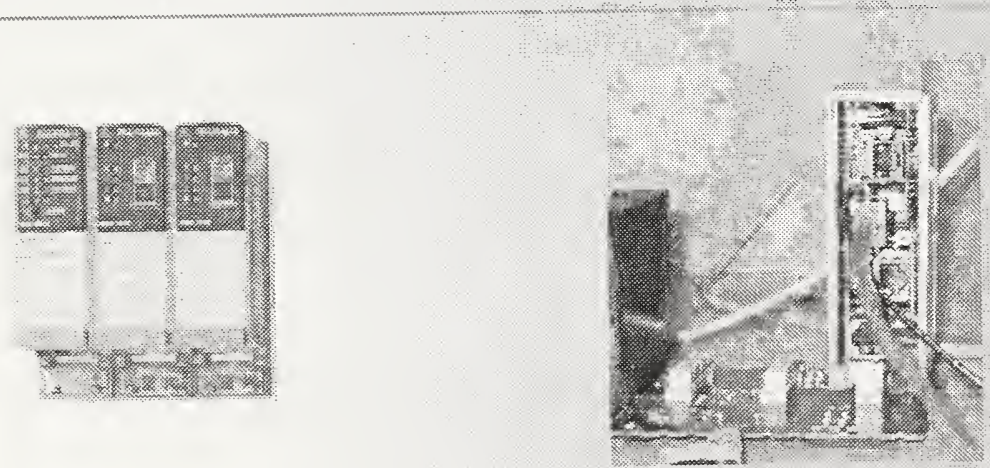

Modular Industrial Ethemet Switch

- new module with 4 IEEE 1588 Ports

- full implementation of IEEE1588 protocol

- time stamp and clock in hardware

- SNMP management 
IEEE 1588 Network

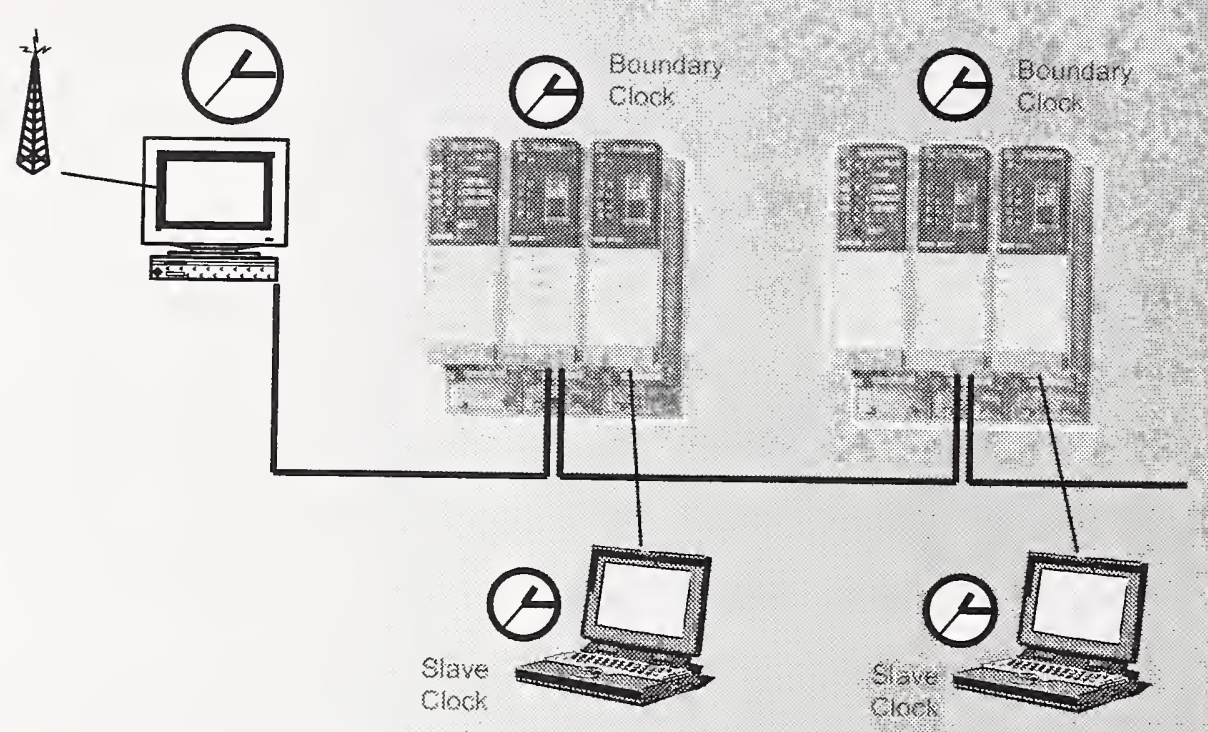

IEEE 1588 Precision Measurement

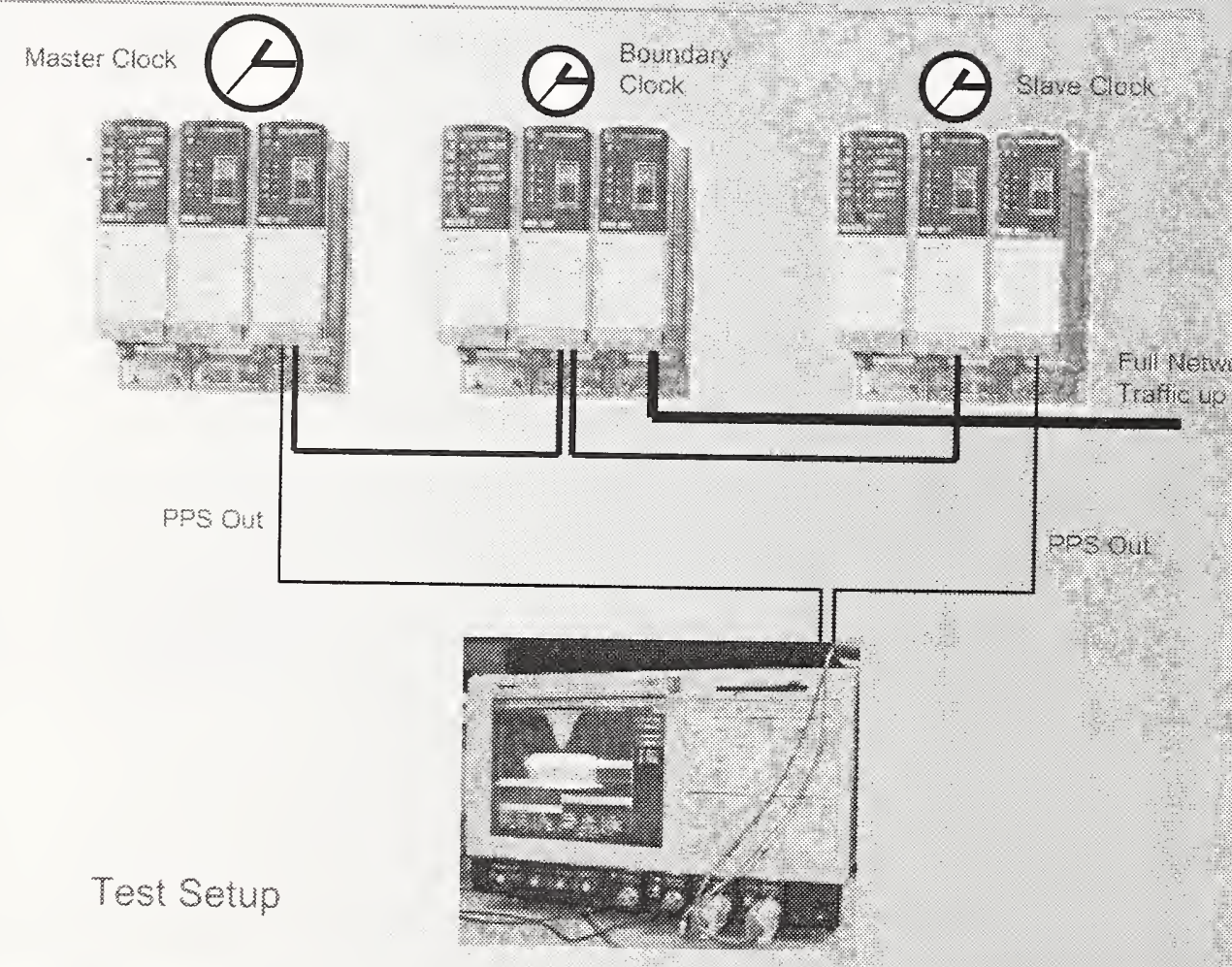




\section{IEEE 1588 Test results}

no special crystals/oscillators, typical network traffic

\section{Devices directly coupled}

$\Rightarrow$ synchronicity about \pm 120 n (approx. 84h)

\section{Cascaded Clocks}

2 devices coupled through a IEEE 1588 switch

$\Rightarrow$ synchronicily about 240 ns.

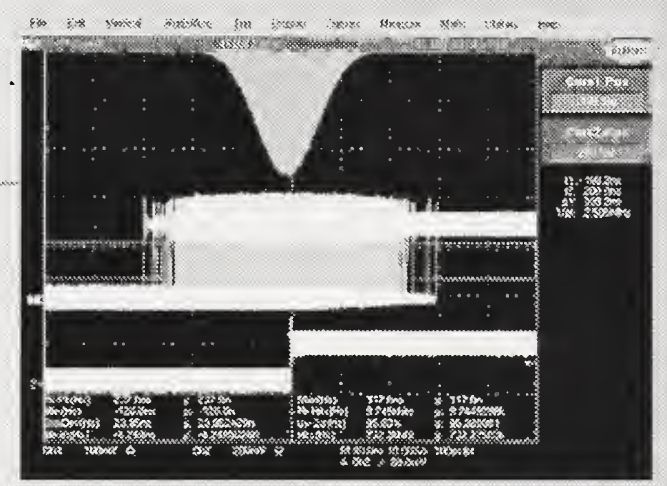

Approximately it can be said, that each cascaded switch adds its clock jitter to the complete transmission line

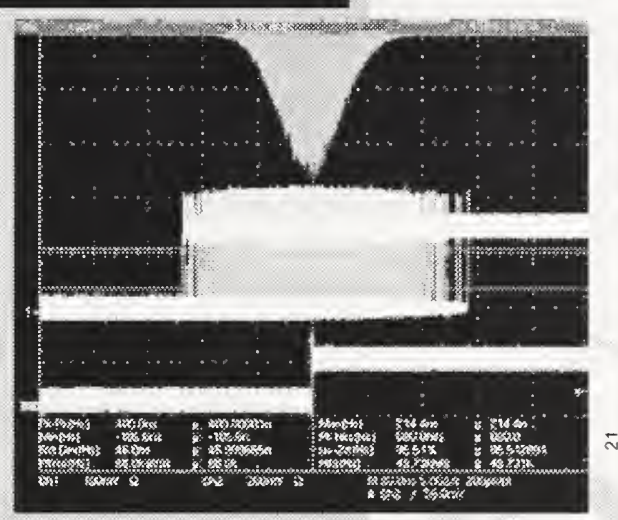

If no special crystals/oscillator are used the system is very sensitive to variation in temperature, even a breath of wind results in a higher clock jitter

IEEE 1588 Test results

\section{Speed of synchronization}

an other very important point is the speed how fast the clock of a slave clock can be adjusted

special control algorithms are necessary if

drift correction and offset correction is implemented

in the current implementation to directly connected clocks reach maximum synchronicity within 60 seconds (IEEE 1588 default configuration)

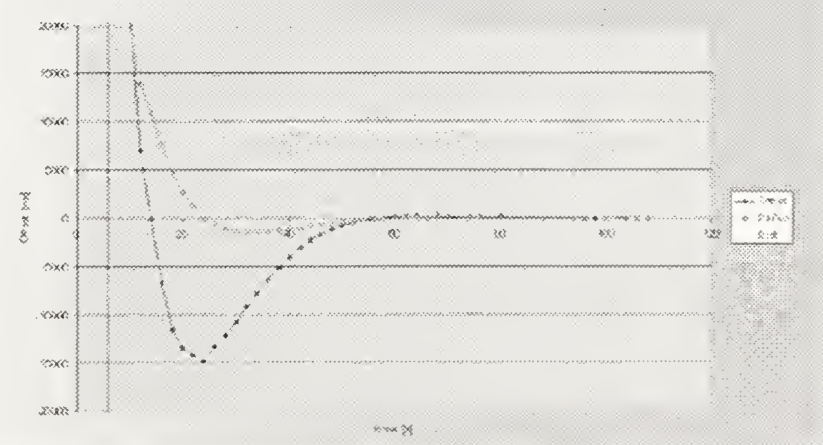




\section{Part 1II: IEEE 1588 - Additions to the Standard}

- Management by SNMP

- Ideas for Improvements of IEEE1588

- IEEE1588 and Ethernet Powerlink (EPSG)

\section{IEEE 1588 Management by SNMP}

\section{Extract of IEEE 1588 MIB}

SNMP is by now the most frequenty used protocol on nework devices.

Since this protocolis already implemented on the switch it is obvious that it is also used to configure relevant IEEE 1588 parameters.

- hmNetwork / ptp grottp (FEEE 1588$)$ -

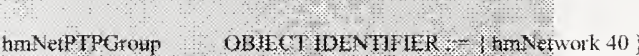
tomPTPConfiguration OBHECT IDENFIFIER = / haNkePTPGroup 1 \}

lamprpetable himPTPActum

hamPTEClockstratuma hamPT PC lockldentifiar hazPTPPieferred Master hmPTPSynclnterval hmPTP'Subdontain Name hmP PnOffset ToNasierNanosecs

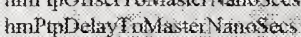
hmPTPParentULis tomP T Tranidnaster thmP TPC enrentu f Cofiset tanPTPleapst hin TPleapol tanPTPEochdiumber fanPTFPort atofe AmPTPPortEntry ! 
IEEE 1588 Enhancements

Higher Synchronization Rate (e.g. 10x)

- faster transient oscillation

- enables the use of oscillators with higher temperature drift

- suitable for small domains (bandwidth)

Adaptive delay request measurements

- faster transient oscillation

- faster reaction to network topology changes

- suitable for small domains (bandwidth)

\section{IEEE 1588 Enhancements}

\section{Detection of error conditions}

- Aging speed of slave ports (10 lost Sync messages)

- Handling of faulty state (e.g. due to missing FollowUp msgs)

- PTP transparent switch / bypass clock

- Idea: measuring the propagation delay of sync messages and delay request messages and correcting corresponding follow up messages and delay response messages with these values

- switch is transparent for IEEE1588 clocks

- no IEEE1588 protocol modification necessary

- switch adds nearly no jitter to clock synchronization

- new protocol on this transparent switch (small)

- does only work if FollowUp service is supported 


\section{IEEE 1588 in Automation}

Intially EEE 1588 was designed for time synchronization in test and measurement applications.

Automation is now very interested in IEEE 1588 :

CIPSync, Promet V3, Ehemel Powerink (EPSC), IEC61850 (Communication networks and systems in substations\}...

Hirschmamn is active in EPSG

Ethernet Powerlink is one solution for Ethernet in Motion Control

- 100\% Ethernet 802.3 compliant

- Cyclic comnunication

- Profile: CANopen

- Segmentation by Gateway

- Time synchromzation $100 \%$ compliant to IEEE 588

Real Time Applications with Ethemet

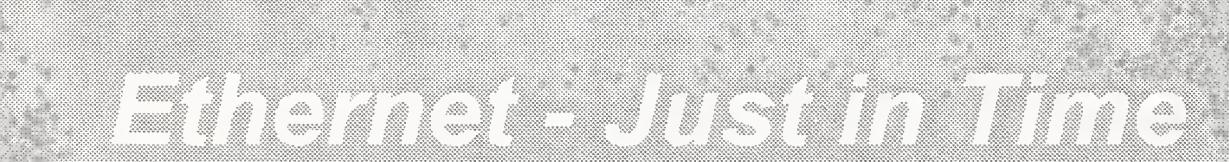

(2.

(3:

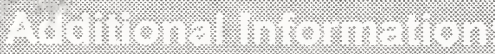

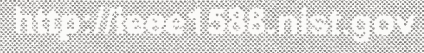

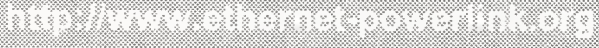

ETHERNET

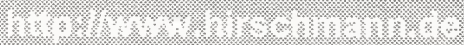

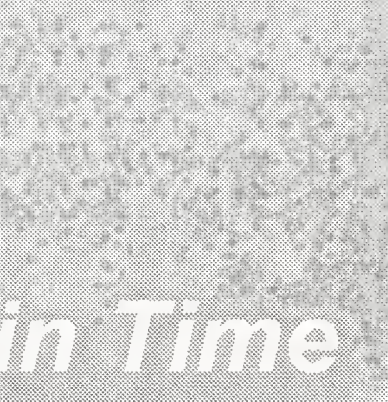

Dirk S. Mohl, Hirschmann Electronics GmbH \& Co.KG, dirk.mohl@nt.hirschmann.de 


\section{A Frequency Compensated Clock for Precision Synchronization using IEEE 1588 Protocol and its Application to Ethernet}

\section{Sivaram Balasubramanian, Kendal Harris and Anatoly Moldovansky}

Rockwell Automation

September 24, 2003

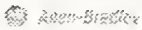

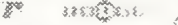

Fis:

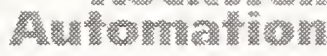

\section{Introduction}

- Clock/oscillator drift

- Temperature, air circulation, mechanical stress, vibration, aging, etc.

- IEEE 1588 protocol

- Standardizes time \& distribution method

- Distributed control

- Managing drift during sync interval

- Frequency Compensated clock

- Simple, fast \& accurate

- Compensates frequency drifts \& clock difference 


\section{Frequency Compensated clock}

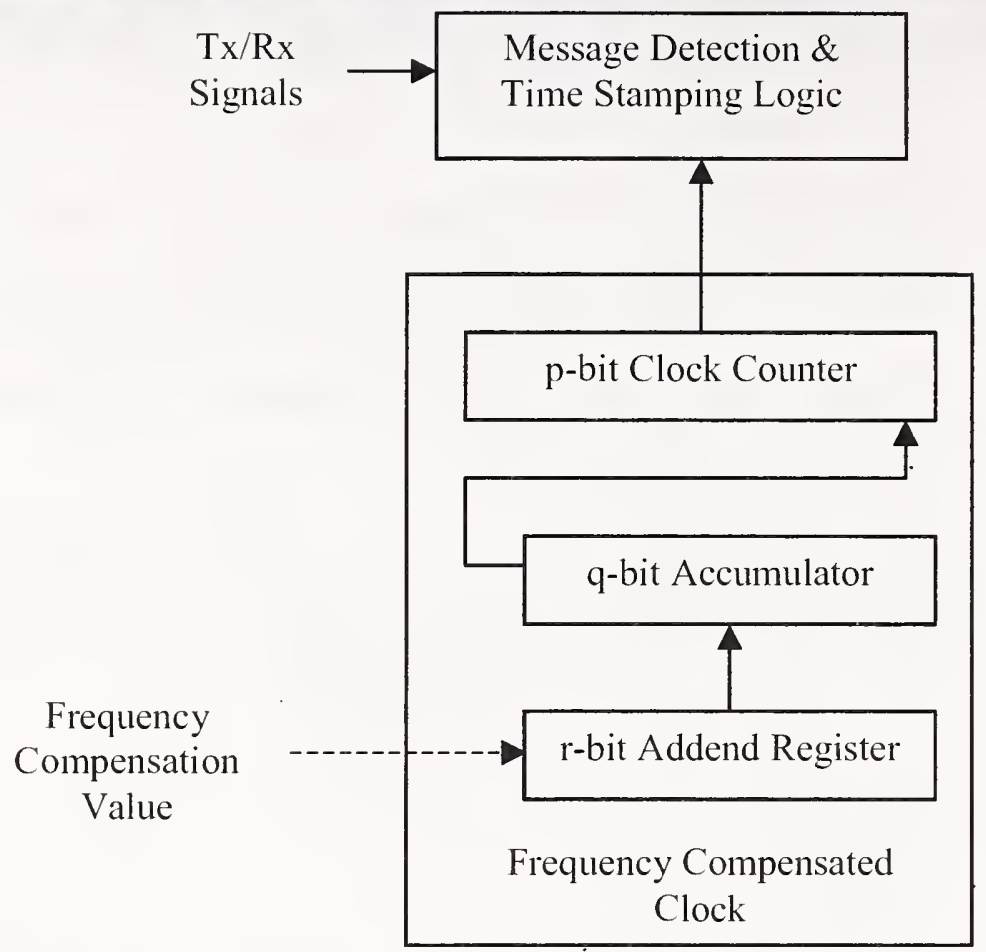

\section{Frequency Divider}

- FreqDivisionRatio $=$ FreqOscillator $/$ FreqClock

- CompensationPrecision $\leq 1$ / (SyncInterval * FreqClock)

- $2^{q} \geq$ FreqDivisionRatio / CompensationPrecision

- $2^{r} \geq 2^{q}$ / FreqDivisionRatio

- $2^{p} \geq 2^{q}$

- For example

- FreqOscillator $=50 \mathrm{MHz}$, FreqClock $=40 \mathrm{MHz}$, FreqDivisionRatio $=1.25$, CompensationPrecision $=$ $1 \times 10^{-9}$, width of accumulator $q=32$, width of addend register $r=32$, width of clock counter $p=64$ 


\section{Frequentr Compensation Algorithm}

- Fast, one sync interval settling time

- Self-correcting behavior, initial accuracy not required

- Initially, FreqCompValue $=29 /$ FreqDivisionRatio

- Algorithm

- MasterClockTime ${ }_{n}=$ MasterSyncTime $_{n}+$ MasterToSlaveDelay

- ClockDiffCount $_{n}=$ MasterClockTime $_{n}-$ SlaveClockTime $_{n}$

- Where,

- $n$-Sync message count

- MasterSyncTime $e_{n}$ - time at which Master sends a Sync message to a Slave

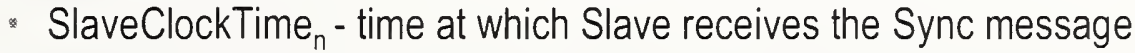

- MasterClockTime ${ }_{n}-$ computed by the Slave after the Sync message is received

\section{Frequency Compensation Algorithm (cont.}

- FreqScaleFactor ${ }_{n}=\left(\right.$ MasterClockCount $_{n}+$ ClockDiffCount $_{n}$ ) / SlaveClockCount

- Where,

- MasterClockCount $t_{n}=$ MasterClockTime $_{n}-$ MasterClockTime $_{n-1}$

- SlaveClockCount $=$ SlaveClockTime $_{n}-$ SlaveClockTime $_{n-1}$

- FreqCompValue ${ }_{n}=$ FreqScaleFactor $_{n}{ }^{*}$ FreqCompValue $_{n-1}$ 


\section{Summary}

- Implementation on 10/100 Mbps switched Ethernet using FPGA

- 25 nanosecond clock resolution with $+/-100$ nanosecond worst case accuracy

- Accuracy limited by non-determinism of PHY devices and switch

- Frequency compensation eliminates most oscillator errors

- Short term stability: Many standard crystal oscillators are short-term stable to few ppb 


\section{IEEE-1588 Node Synchronization Improvement by High Stability \\ Oscillators}

\section{John Eidson \& Bruce Hamilton}

-Sources of fluctuation in an IEEE 1588 system - Characterization of oscillators

-Measurement results

-Conclusions

Work supported by U. S. Naval Research Laboratory- Dr. Joe White (John Eidson, Bruce Hamilton- Agilent; Mike Fischer- Fischer Consulting) 


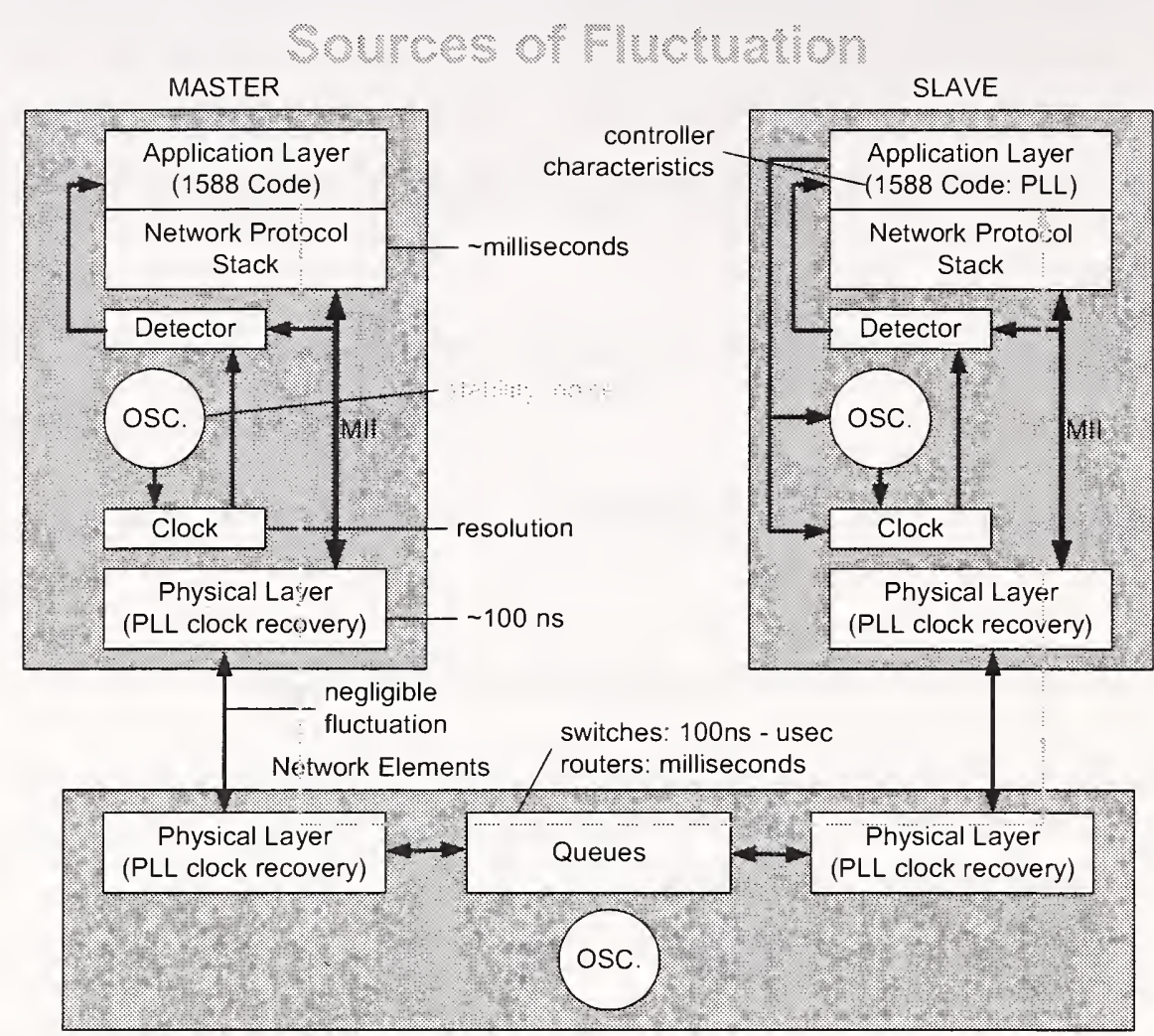

\section{Characterization of oscillators}

- Long term systematic effects: aging

- Short term systematic effects:

- temperature, pressure, operating voltage

- Short term random processes: Allan variance 


\section{Allan frequency deviations for test \\ oscillators}

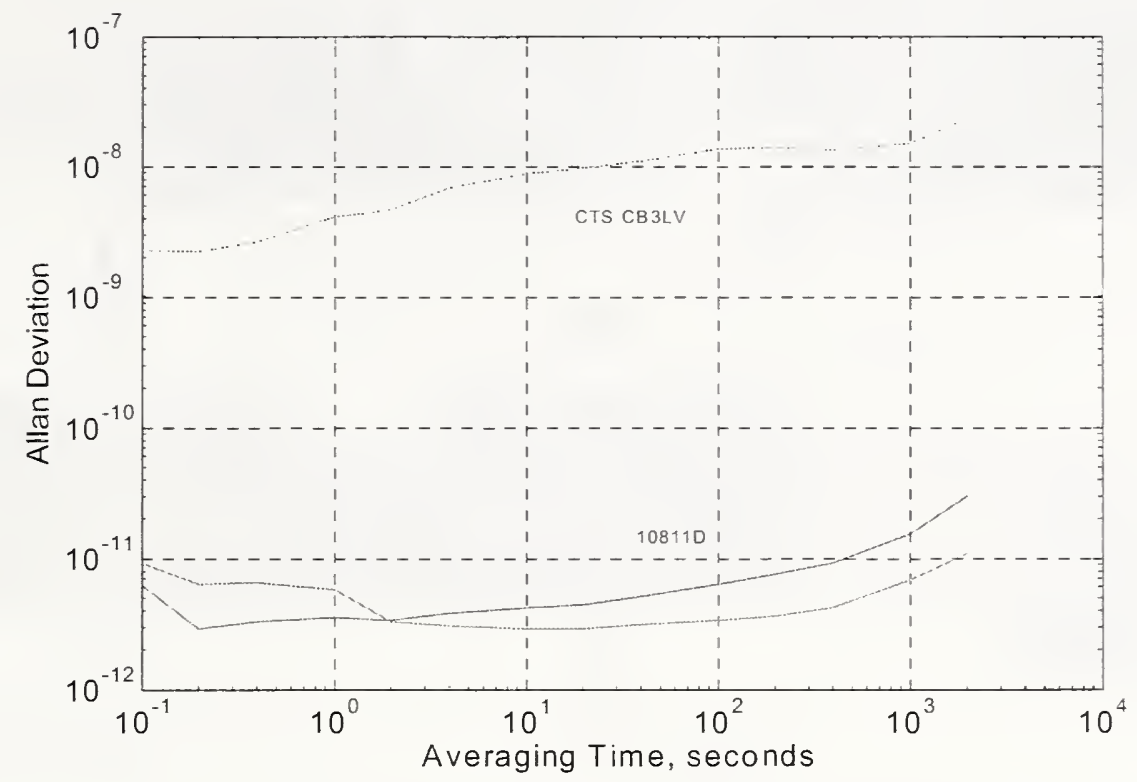

\section{Measurement setup lo test prototypes of an earler}

\section{version of 1588}

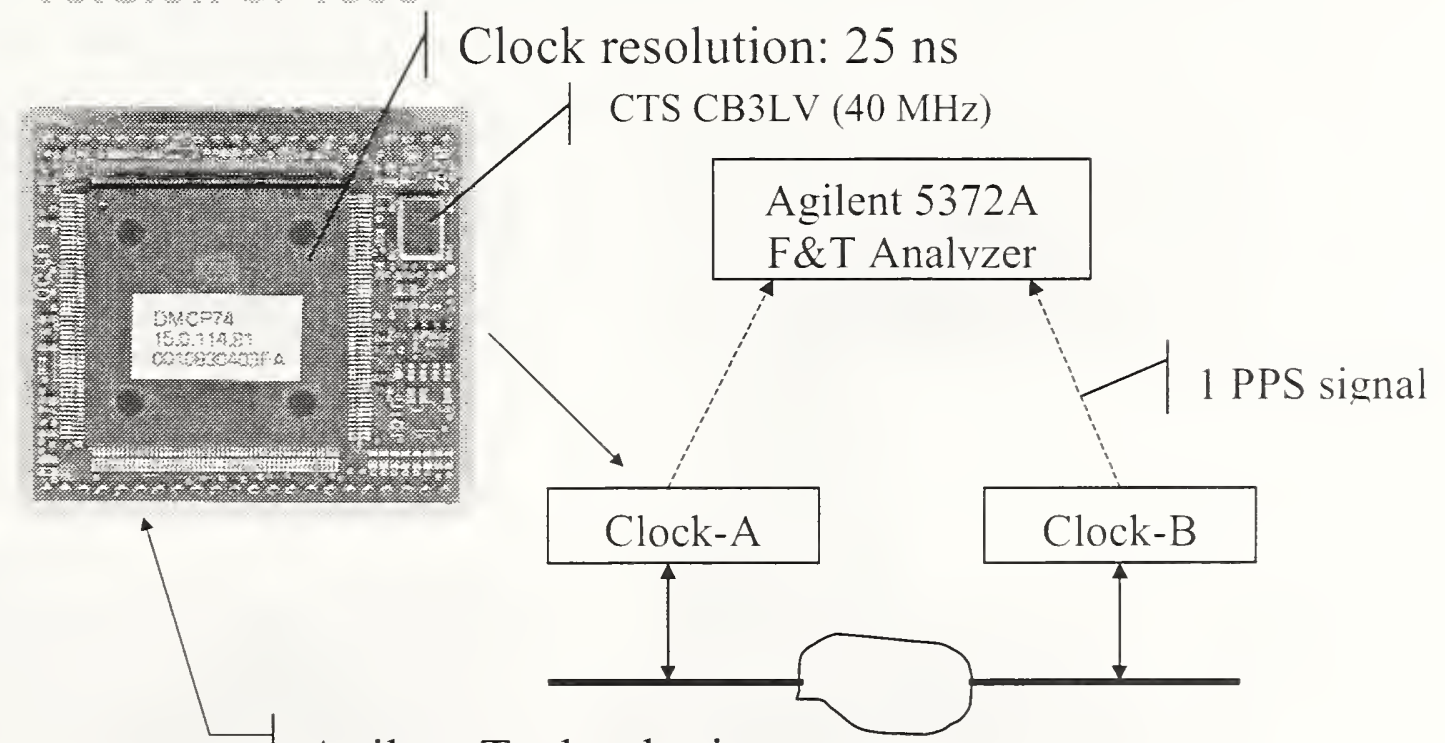

Agilent Technologies prototype clock 


\section{IEEE 1588 Topologies}

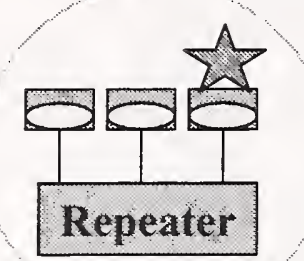

$\checkmark=1588$ clock, code \& hardware

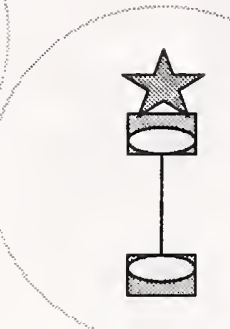

Grand Master Clock

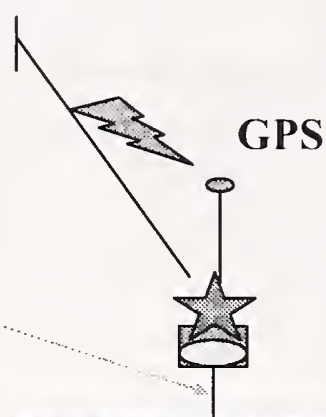

Slave Clock
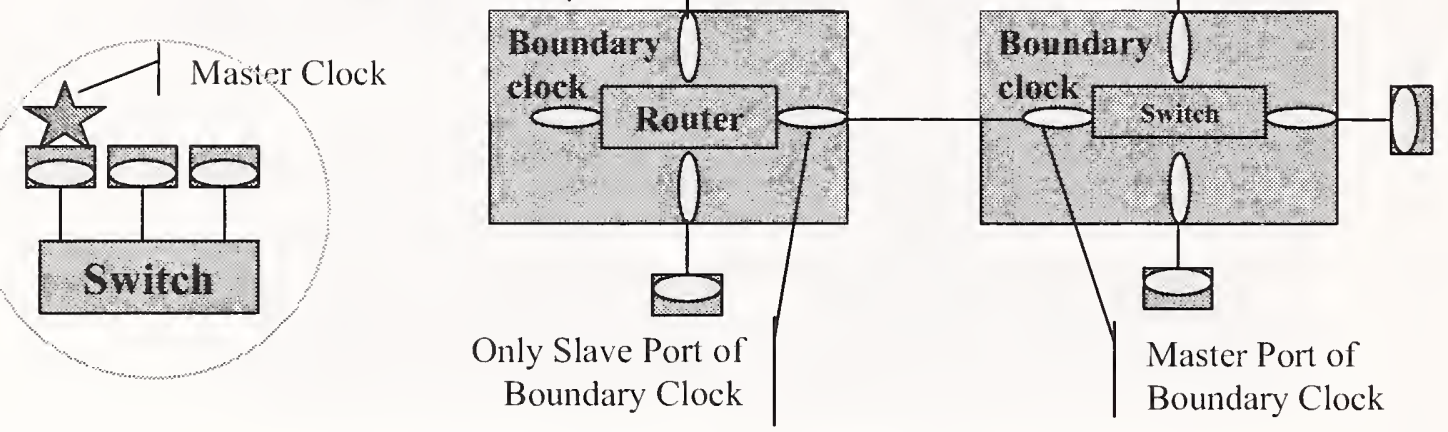

IEEE1588 Workshop September 24, 2003

\section{Switch Connection (1 hour)}
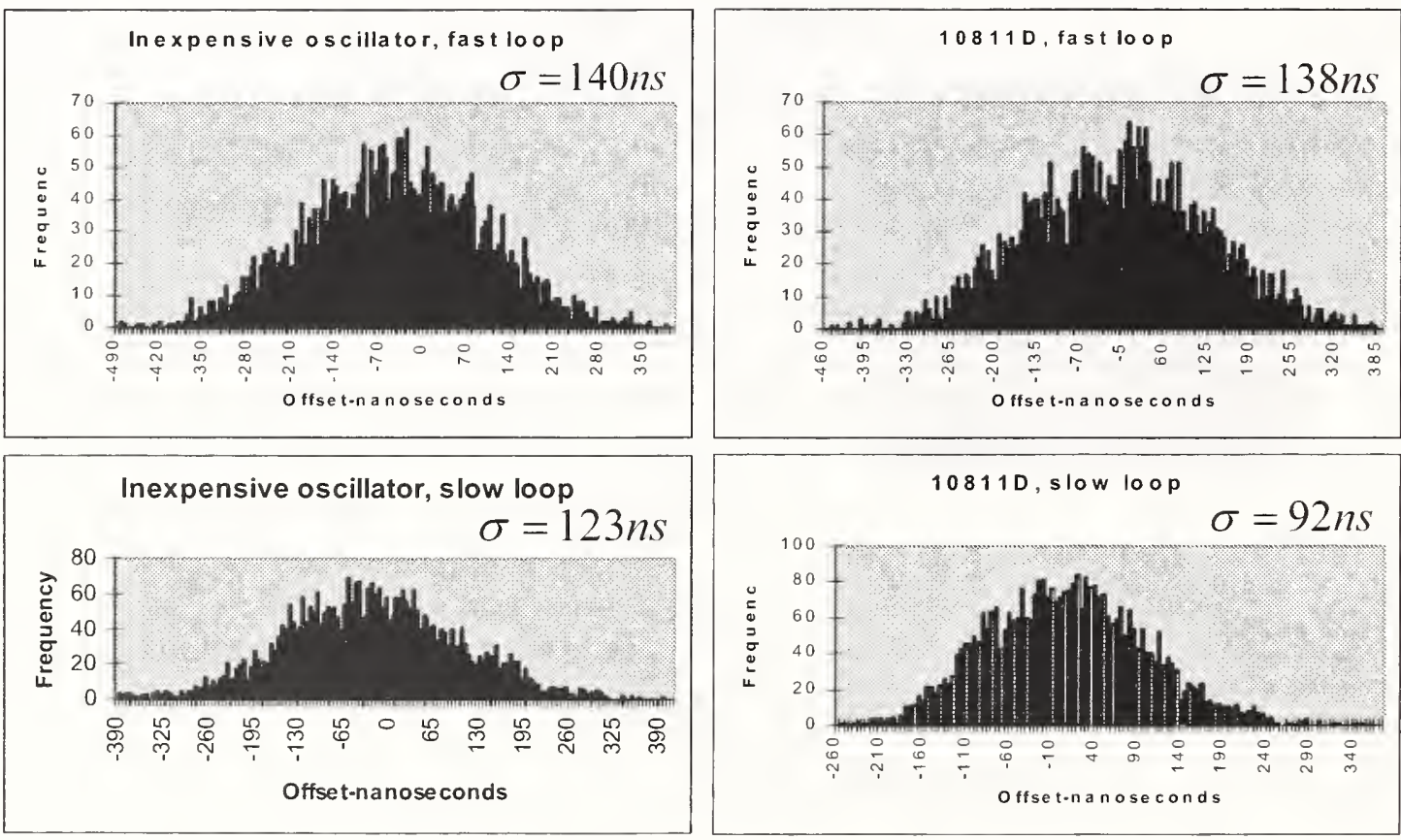


\section{Repeater Connection (1 hour)}
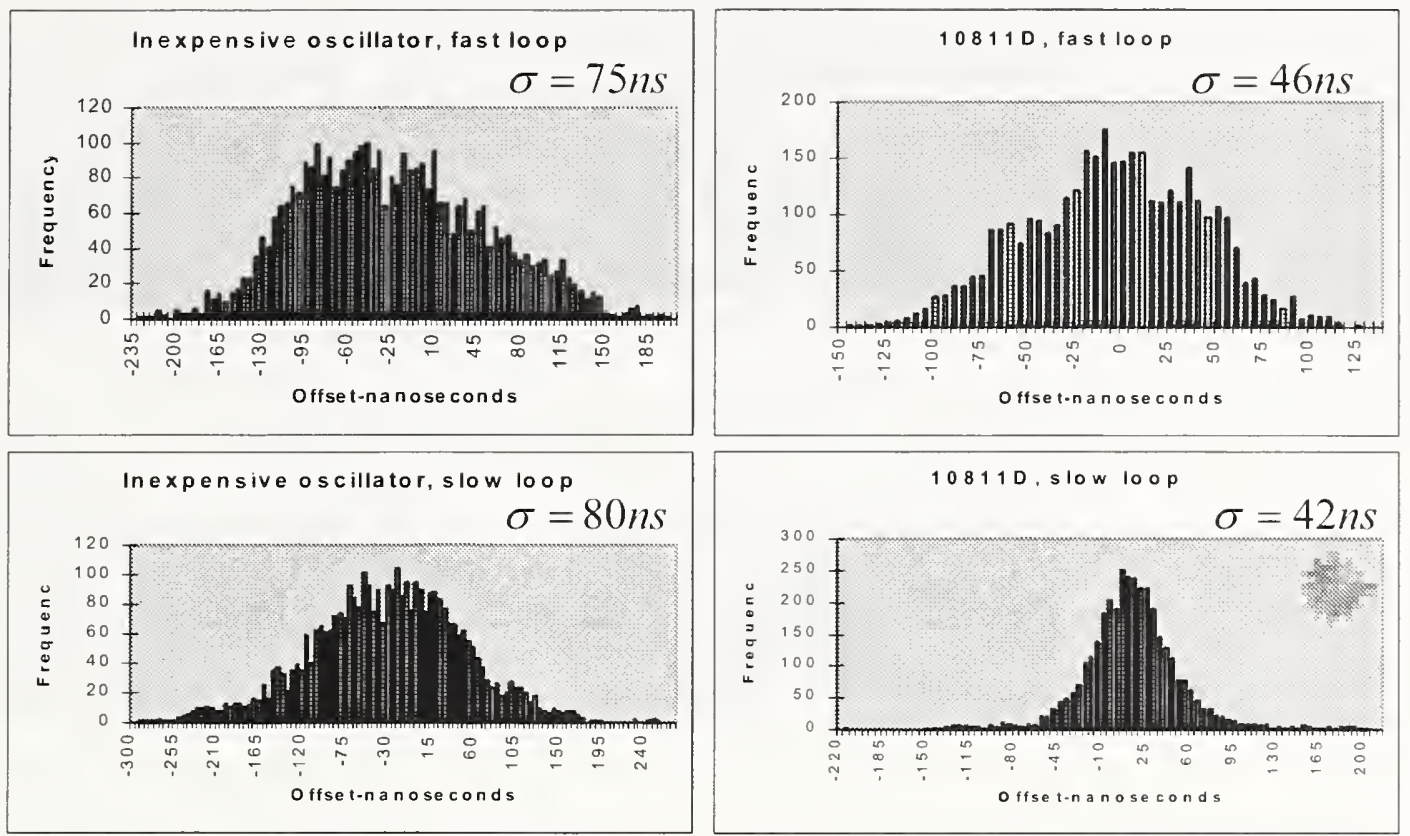

\section{Seconds tick deviations: connection via a repeater-72 hours}

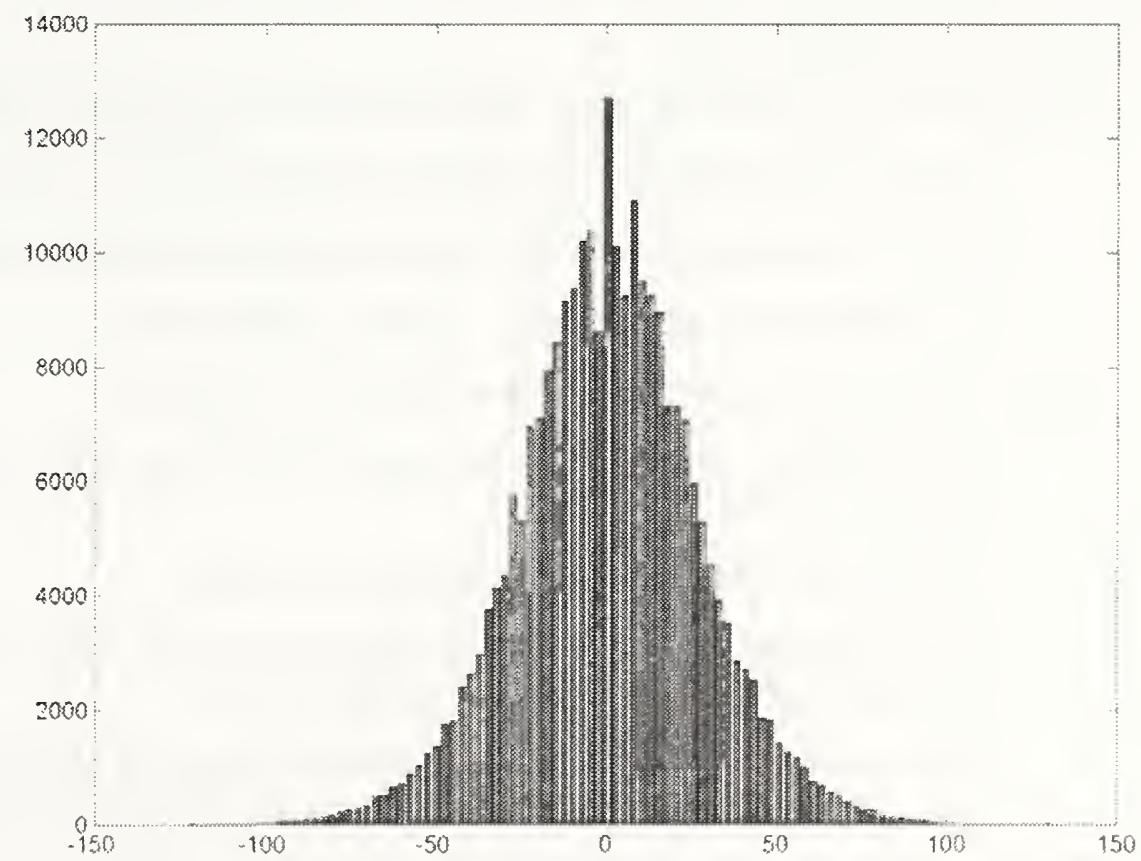

mean: 801 ps Std. Dev: $26.8 \mathrm{~ns}$

IEEE1588 Workshop September 24. 2003

\section{nanoseconds}

Agilent Technologies

Page 10 


\section{Direct Connection}
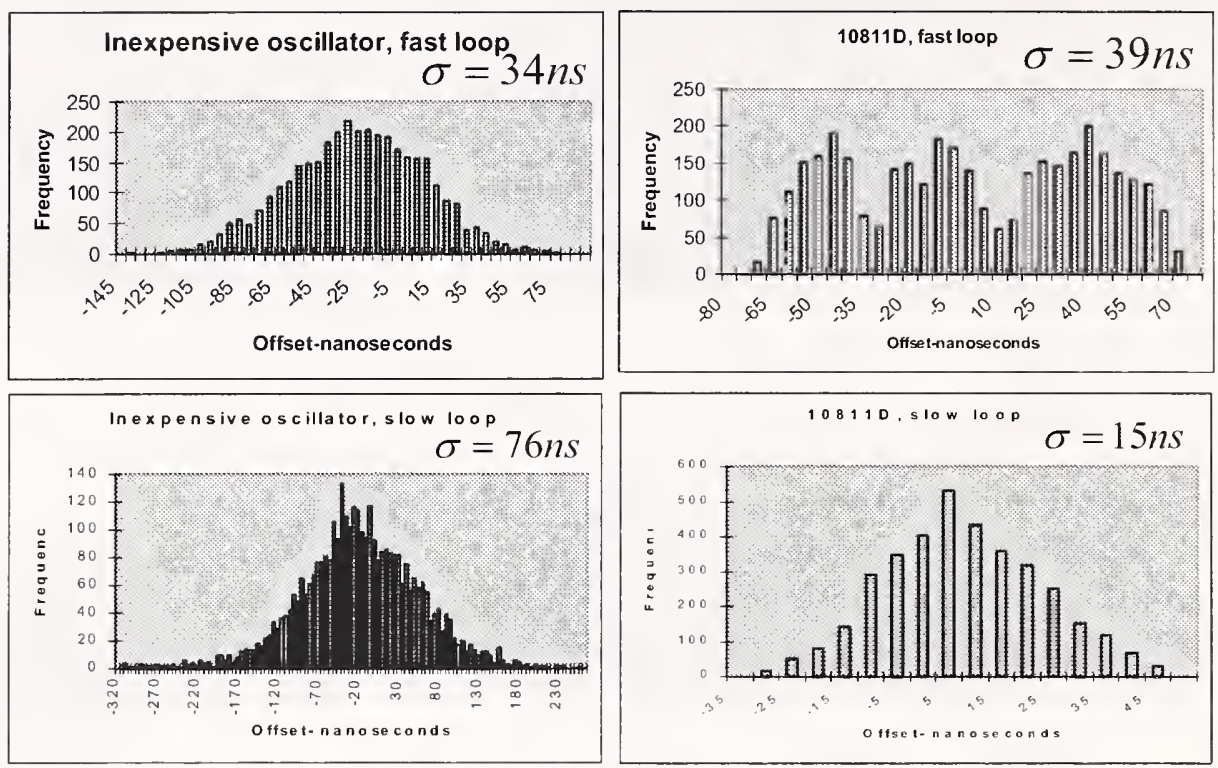

\section{Summary of effects of oscillator}

\section{characteristics}

-Inexpensive oscillators: poor temperature \& noise properties

- Limit integration and statistical techniques in slave controllers

- Shorter synchronization intervals use bandwidth and computation

- If in master clock produce unstable time base

-High quality, temperature compensated oscillators:

- Allow longer integration times and use of statistical techniques in slave controllers

- If in master clock produce stable time base

- Provide good holdover which allows more latitude in handling communication failure or reconfiguration

- Reasonably priced oscillators should support clock to clock synchronization limited by clock resolution to $\sim 10$ nanoseconds 


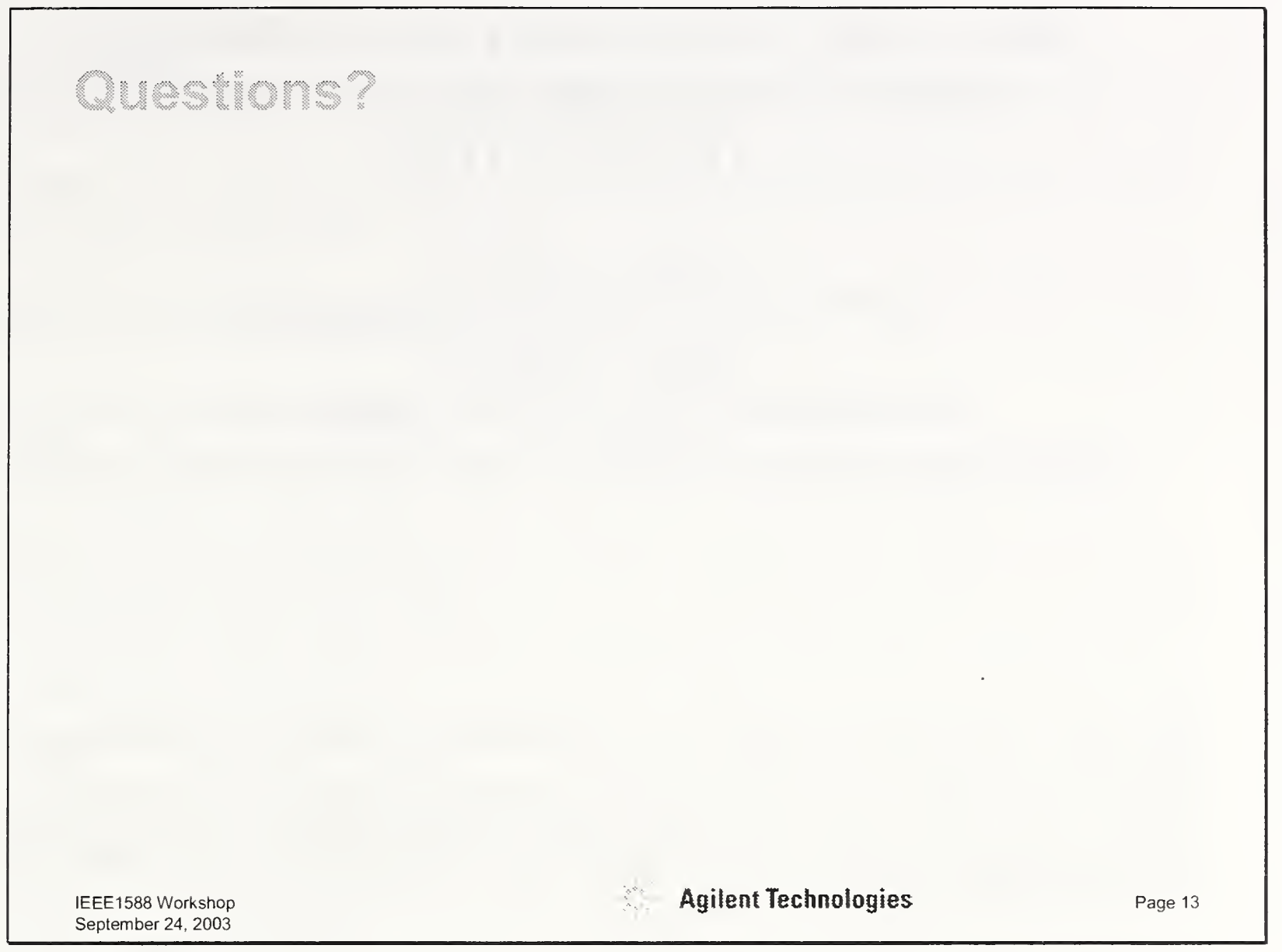




\title{
IEEE-1588 ${ }^{\text {TM }}$ NODE SYNCHRONIZATION IMPROVEMENT BY HIGH STABILITY OSCILLATORS
}

\author{
John C. Eidson \\ 650-485-4263, john eidson@agilent.com \\ Bruce Hamilton \\ 650-485-2818, bruce_hamilton@agilent.com \\ Agilent Laboratories, 3500 Deer Creek Rd., Palo Alto, CA, 94304
}

\begin{abstract}
This paper outlines work done for the US Naval Research Laboratory to investigate the sensitivity of synchronization accuracy with the stability of local oscillators in IEEE 1588 systems. Performance results of prototype implementations of this standard in an Ethernet environment will be presented. The implications of this work on IEEE 1588 design will be discussed.
\end{abstract}

\section{INTRODUCTION}

In most measurement and control systems execution timing is determined by the design of electronic components and the implicit timing in computer programs. As the systems being measured or controlled increase in size and complexity it becomes increasingly difficult to manage timing constraints in this fashion. This is particularly true when the communication between devices and controllers is via a network.

Recently there has been increased interest in making time explicit in such systems as a way to improve timing performance. This has been common practice in the general computer environment but generally with looser timing constraints than found in typical measurement and control systems. Such networked systems are typically implemented with a real-time clock in each node and with the suite of clocks synchronized via some protocol. In the general computing world the dominant protocol is the Network Time Protocol, NTP [5]. IEEE-1588-2002, 'Standard for a Precision Clock Synchronization Protocol for Networked Measurement and Control Systems' was designed to serve the clock synchronization needs of measurement and control systems. Typical applications and their required accuracies are listed in Table 1.

Table 1: Typical application synchronization requirements

\begin{tabular}{|l|l|}
\hline \multicolumn{1}{|c|}{ Application area } & $\begin{array}{l}\text { Required synchronization } \\
\text { accuracy }\end{array}$ \\
\hline Low speed sensors (e.g. pressure, temperature) & Milliseconds \\
\hline $\begin{array}{l}\text { Common electro-mechanical devices (e.g. relays, breakers, } \\
\text { solenoids, valves) }\end{array}$ & Milliseconds \\
\hline $\begin{array}{l}\text { General automation (e.g. materials handling, chemical } \\
\text { processing) }\end{array}$ & Milliseconds \\
\hline $\begin{array}{l}\text { Precise motion control (e.g. high speed packaging, printing, } \\
\text { robotics) }\end{array}$ & A few microseconds \\
\hline
\end{tabular}




\begin{tabular}{|l|l|}
\hline $\begin{array}{l}\text { High speed electrical devices (e.g. synchrophasor } \\
\text { measurements) }\end{array}$ & Microseconds \\
\hline Electronic ranging (e.g. fault detection, triangulation) & Sub microsecond \\
\hline
\end{tabular}

This paper discusses some of the practical considerations in implementing IEEE 1588 needed to meet the most demanding timing requirements.

The U. S. Naval Research Laboratory sponsored much of the work reported here. A more complete report of this work may be found in [1].

\section{High ACCURACY Operation OF The IEEE-1588 Protocol}

Within a network subnet the IEEE-1588 protocol establishes a master-slave relationship among the participating clocks. The master is selected as the best clock based on defined descriptors maintained by cach clock describing inherent accuracy, traceabilty to UTC, inherent variance, etc. A properly designed IEEE 1588 system produces a self-consistent, system-wide time base closely synchronized to the master clock. Since the slave nodes operate a servo to synchronize their local clock to the clock of the master, the stability and noise properties of the master limit the overall time performance of the system. If the time base of the system is to be UTC then the master clock must maintain or be synchronized to an appropriate source of UTC time.

To obtain high accuracy the slaves synchronize their local clocks to that of their master by an exchange of messages illustrated in Figure 1. Periodically the master clock sends a distinguished message, a Sync message, as a multicast to all its slaves. The master implements mechanism for detecting and time stamping the time that the Sync message is actually placed on the nctwork based on the master's local clock. The master's IEEE-1588 code sends this measured time stamp, the actual sending time stamp, to all slaves in a second message, the Follow_up message. The slaves receive the Sync message and detect and time stamp its arrival as close to the network as possible. Upon receiving the Follow_up message the slave's IEEE-1588 code uses the contained actual scnding time stamp and the local receipt time for the Sync message to correct the time of the slave's local clock. Periodically, but with longer period to reduce network loading, this process is reversed. This forward and reverse path information is used to compute the onc-way network latency on the assumption that the path is symmetrical. The slaves use this measured latency in computing the correction to their local clock. This procedure effectively removes the latency in the communication path.

\section{Sources Of Timing Fluctuations In A IEEE 1588 System}

There are several sources of timing fluctuations in an IEEE 1588 system. These are illustrated in Figure 2 for an Ethernet implementation. The dashed line shows the communication path taken by the Syne messages between the IEEE 1588 code in the master and the slave. The primary sources of fluctuation are found in network components and in the end devices themselves. Fluctuations in the actual network media are gencrally negligible for the target applications of IEEE 1588 although this may not hold if there are wireless links involved.

A major source of timing errors is network latency fluctuations introduced by network elements such as repeaters, switches, and routers. Routers introduce fluctuations too large and inconsistent to be reduced to the desired accuracy with statistics. IEEE-1588 specifies a transfer standard mechanism, the boundary clock, for logically eliminating routers from the IEEE-1588 protocol communication path. Modern switches implement level 2 protocols that can further reduce fluctuations based on the priority of Sync messages [2]. It is also possible to design switches incorporating IEEE-1588 boundary clocks that logically remove them from the IEEE-1588 communication paths. 
A boundary clock appears to each subnet of interest as an ordinary IEEE-1588 clock as provided in an end device. A boundary clock uses a single local clock to scrve as a master in either all or all but one of the subnets of interest. As a result boundary clock equipped routers and switches appear to a link as two end devices as far as clock synchronization is concerned. This technique effectively produces direct synchronization between the clocks in the end device and the clock in the router or switch. In cases where multiple boundary clocks are needed in an application, the boundary clocks themsclves form a hierarchy so that in a properly implemented IEEE-1588 system there is always a single clock serving as the primary source of time for the ensemble.

In the restricted environments typical of the target applications, the fluctuations in repeaters and in many cases switches can generally be reduced to acceptable levels by the use of statistical techniques amcnable to low cost deviccs. Further details on these mechanisms and other features of IEEE-1588 beyond the scope of this paper may be found in the standard itself or on the IEEE1588 web site [3].

The other major source of timing fluctuations is in the end devices, or the individual ports of a boundary clock. Within such a device the worst fluctuations occur in the network protocol stack and opcrating system usually due to the queuing of messages and context switching. In an Ethernet node these fluctuations are avoided by detecting both incoming and outgoing Sync messages as close to the physical layer, the PHY, as possible. The proper usc of interrupt service routines can help, but for the highest accuracy some sort of hardware assist is required. The MII interface is a natural place to implement this function as illustrated in Figure 2.

Additional sources of fluctuation within a node are the PHY, the oscillator, and in the slave the servo. PHY chips introduce a low lcvel of fluctuation primarily due to the phasc lock loop, PLL, used for clock recovery. These fluctuations are amenable to statistical averaging. A second PLL exists in each slave node implementing the servo to synchronize the slave clock to the master. The design of this servo is also sensitive to the stability and noise properties of the oscillators in both the slave and the master.

\section{The Effect Of Local Oscillators On IEEE 1588 Performance}

The timc base of a local clock in an IEEE 1588 system is derived from some sort of local oscillator. In low cost end devices this oscillator will often be an inexpensive crystal oscillator that also serves as the clock source for a microprocessor. Since the IEEE 1588 protocol specifies a sampled data system any drift or fluctuations of these oscillators between samples cannot be corrected by the slave clock's scrvo. These drift and fluctuations also limit the effective avcraging times of the slave's PLL thus limiting the ability of the slave servo average out fluctuations due to any network components. In addition the stability of the oscillator in the master clock determines the overall time stability of the system. No servo can track the master clock perfectly so the drift rate of the master clock must be controlled in high accuracy situations. The absolutc frequency accuracy of the oscillators is critical only insofar as it influences the dynamic range over which the local servo must be prepared to operatc. IEEE 1588 specifies $0.01 \%$ absolute frequency accuracy, the same as required to ensure operation of the 10 BaseT Ethernet protocol.

All oscillators are subject to frequency errors. These errors may be categorized as long and shorttcrm systematic effects, and as various random processes. Long-term systematic effects, usually termed aging, are not of concern in the design of an IEEE 1588 system since servo time constants will usually be on the order of seconds. Short-term systematic effects result from the sensitivity of oscillator frequency to temperature, pressure, supply voltage, etc.

Of these temperature is the most difficult to control in the target environments. Since the scrvo must track any temperature induced drift of the oscillators this thermal drift must be controlled. For example with a timing accuracy specification of 1 microsecond, a sampling time of 2 
seconds, and a 1 PPM temperature cocfficient for the oscillator the thermal time gradient must be held to less than 0.5 degree per second at the oscillators. There are only three ways to improve this situation. The first is to decrease the sampling interval. The standard currently specifies a default interval of 2 seconds and a minimum interval of 1 second. These were chosen as a compromise between responsiveness and network loading. Secondly oscillators with better temperature coefficients may be selected. Cheap oscillators tend to be very poor in this regard, often $10-50 \mathrm{ppm} / \mathrm{deg} \mathrm{C}$. The use of temperature compensated oscillators, TXCOs, can provide between 0.3 and $1.0 \mathrm{ppm} / \mathrm{deg} \mathrm{C}$ with moderate costs. Oven controlled oscillators are at least an order of magnitude better and can be used for the most demanding circumstances. Finally careful thermal design can minimize the thermal drift rates. It is the drift rate with time rather than the absolute frequency that is the issue. A side benefit of improved thermal performance is better holdover time in the event of communication failure.

All oscillators are also subject to various random processes manifesting in frequency fluctuation. The spectral characteristics and absolute magnitudes of these fluctuations differ markedly between various forms of oscillators. For the target environments most oscillators will be quartz crystals due to their lower cost compared to more stable oscillators such as rubidium or cesium. Thesc oscillator fluctuations are characterized by the Allan variance [4].

Figure 3 illustrates the Allan frequency variance for two types of oscillators. Data derived using two different grades of oscillators is reported. The CTS CB3LV, an inexpensive $40 \mathrm{MHz}$ oscillator, is typical of quartz oscillators used in very cost sensitive applications. The 10811D oscillators are ovenizcd, instrument grade quartz oscillators. For the $10811 \mathrm{D}$ oscillators the 10 $\mathrm{MHz}$ oscillator frequency was quadrupled to the needed $40 \mathrm{MHz}$ by use of a PLL.

Based on the data in Figure 3 the expected time fluctuations introduced by the oscillators are shown in Table 2 for several values of the averaging time $\tau$.

Table 2: Expected oscillator induced time fluctuation

\begin{tabular}{|l|l|l|}
\hline$\tau$ - seconds & \multicolumn{2}{|c|}{ Fluctuations- nanoseconds } \\
\hline & Inexpcnsive & $10811 \mathrm{D}$ \\
\hline 1 & 4 & 0.006 \\
\hline 2 & 9.2 & 0.006 \\
\hline 10 & 90 & 0.040 \\
\hline 100 & 1400 & 0.700 \\
\hline
\end{tabular}

From Figure 3 and Table 2 it is apparent that for synchronization accuracies in the submicrosecond range and with averaging times on the order of seconds that oscillators with about an order of magnitude better performance than the inexpensive oscillator should be selected.

\section{IEEE-1588 PERFORMANCE EXPERIENCE}

This section discusses performance measurements on a prototype implementation of IEEE-1588 under varying network topologics. The measurements are made using the experimental configuration shown in Figure 4. The ASIC shown contains a 68020 class $40 \mathrm{Mhz}$ processor and a Sync message detector observing the MII interface as illustrated in Figure 2. $40 \mathrm{MHz}$ crystal oscillators drive the hardware clocks to provide a real-time clock with a resolution of 25 ns. Performance data is reported for samples of these prototype clocks driven by the inexpensive oscillators and by the $10811 \mathrm{D}$ oscillators described in Figure 3.

Each clock has a 1 PPS test point that rolls over at the seconds boundary of the clock. An Agilent 5372A Frequency and Time Analyzer is used to measure the relative offsets between the 1 PPS signals of the master and slavc. In each casc the data represents statistics on 3600 measurcments made over a one-hour period. 
The prototypes use a simple PI control loop in the slave clocks to servo the local clock to that of the master. The clock offset errors are sampled every 2 seconds as the basis for the PI loop input. Two sets of parameters for the proportional and integral terms in the PI loop are reported. A fast loop set with $\mathrm{P}=2$ and $\mathrm{I}=0.5$, and a slow loop set with $\mathrm{P}=0.5$ and $\mathrm{I}=0.125$. The fast loop has relatively high gain, wide bandwidth and fast response time, a desirable condition during startup to speed acquisition of lock and to drive large initial errors to small values, typically on the order of a minute.

Synchronization results are given for several network connection topologies as illustrated in Figure 5. IEEE-1588 is cxpected to find most extensive application in topologies consisting of a single subnet. This is illustrated in Figure 5 by the collection of three clocks communicating via a repeater or a switch. When multiple subnets are required then for the highest accuracy routers must implement IEEE-1588 boundary clock specifications. Switches may also implement boundary clocks as illustrated. When boundary clocks are uscd then end nodes effectively synchronize directly to their master clock. Results follow for the three basic topologies shown enclosed in circles in Figure 5.

\section{DIRECT CONNECTION BETWEEN CLOCKS}

To show the limiting characteristics of the clock implementation free from fluctuations introduced by network components clocks were tested using a direct connection, e.g. connected via a crossover cable rather than via a repeater or switch. This case also represents the expected performance for topologies in which end dcvices interact directly with a switch, or any other device, implementing IEEE-1588 boundary clock functionality.

Figure 6 contains histograms of the synchronization error for two clocks directly connected via Ethcrnet for both inexpensive and 10811D oscillators and for the slow and fast loop parameters. No fluctuations from the 10811D oscillators are significant for the range of parameters used as noted in Table 2. With the inexpensive oscillators and the fast loop (a few seconds time constant) the clocks manage to track the oscillator induced fluctuations well enough to keep the offsets reasonably well bounded as illustrated by the width of the histogram. However with the slow loop the fluctuations of the inexpensive oscillators bccome more apparent as scen in the incrcased width of the histogram. The means and standard deviations of the measurcments in Figure 6 are shown in Table 3. With the 10811D oscillators one would expect the standard deviations to improve in implementations with clock resolution finer than the $25 \mathrm{~ns}$, and with a true VCO rather than the digital version of the reported implementation.

Table 3: Offset error statistics for direct connection

\begin{tabular}{|l|l|l|l|}
\hline Loop speed & Oscillators & Mean-ns & Std.Dev-ns \\
\hline \multirow{2}{*}{ Fast loop } & Inexpcnsivc oscillators & -28 & 34 \\
\cline { 2 - 4 } & 10811 D oscillators & -1 & 39 \\
\hline \multirow{2}{*}{ Slow loop } & Inexpensive oscillators & -21 & 76 \\
\cline { 2 - 4 } & 10811 D oscillators & 5 & 15 \\
\hline
\end{tabular}

\section{Clocks CONNECTED ViA A REPEATER}

For these mcasurements the clocks were connected via a single HP J4090A Ethernet repeater. No traffic other than synchronization messages was present on the subnet.

Figure 7 contains histograms of the synchronization error for the repcatcr connected clocks for both inexpensive and 10811D oscillators and for the slow and fast loop parameters. The means and standard deviations of the measurements in Figure 7 are shown in Tablc 4. 
Table 4: Offset error statistics for connection via a repeater

\begin{tabular}{|l|l|l|l|}
\hline Loop speed & Oscillators & Mean-ns & Std.Dev-ns \\
\hline \multirow{2}{*}{ Fast loop } & Inexpensive oscillators & -27 & 75 \\
\cline { 2 - 4 } & 10811 D oscillators & -7 & 46 \\
\hline \multirow{2}{*}{ Slow loop } & Inexpensivc oscillators & -32 & 80 \\
\cline { 2 - 4 } & 10811 D oscillators & 10 & 42 \\
\hline
\end{tabular}

In this case the repeater introduced measurable fluctuations for all cases resulting in the increase in the widths of the histograms compared to the corresponding cases for the direct connection. To first order repeater connections will be relatively traffic independent since synchronization messages suffering collisions and retransmission will bear a time stamp for only the successful transmission.

\section{Clocks CONNECTED VIA A SWITCH}

For these measurements the clocks were connected via a single HP J4121 A Ethernet switch. No other traffic other than synchronization messages was present on the subnet.

Figure 8 contains histograms of the synchronization error for the switch connected clocks for both inexpensive and 10811D oscillators and for the slow and fast loop parameters. The means and standard deviations of the measurements in Figure 8 are shown in Table 5.

Table 5: Offset error statistics for connection via a switch

\begin{tabular}{|l|l|l|l|}
\hline Loop speed & Oscillators & Mean-ns & Std.Dev-ns \\
\hline \multirow{2}{*}{ Fast loop } & Inexpensive oscillators & -49 & 140 \\
\cline { 2 - 4 } & $10811 \mathrm{D}$ oscillators & -14 & 138 \\
\hline \multirow{2}{*}{ Slow loop } & Inexpensive oscillators & -21 & 123 \\
\cline { 2 - 4 } & $10811 \mathrm{D}$ oscillators & 5 & 92 \\
\hline
\end{tabular}

Comparing the data for the repeater and the switch, the switch clearly introduces more fluctuations. Unlike repeaters, switches will show sensitivity to traffic although improvement is possible using both priority features of modern switches and more sophisticated statistical treatment of measured transit times of the synchronization messages. These techniques will only be effective when the underlying stability of the oscillators permits statistical treatment of synchronization messages spanning several sampling intervals.

\section{SUMMARY}

In summary inexpensive oscillators have poor temperature and noise properties that limit the synchronization accuracy of an IEEE-1588 system. In particular poor oscillators limit the allowable integration times and statistical techniques used in the phase lock loops of the slave clock controllers. These effects may be somewhat offset by shorter sampling intervals but at considerable cost in network traffic and computational resources.

High quality temperature compensated or controlled oscillators allow longer integration times and more sophisticated statistical techniques to be used in the slave clocks. In addition a high quality clock in the grandmaster clock node will produce a much more stable time basc. An additional benefit is that higher quality clocks generally will allow more design freedom in implementing holdover strategies used in the event of communication failure or reconfiguration.

\section{REFERENCES}

[1]: "Node Synchronization Improvement by High Stability Oscillators" Michael C. Fischer, John C. Eidson, Bruce Hamilton. Agilent Laboratories Technical Report AGL-2002-13. 
[2]: 'Utilization of Modern Switching Technology in EtherNet/IPTM Networks', Anatoly Moldovansky, RTLIA 2002, $1^{\text {st }}$ Int'l Workshop on Real Time LANS in the Internet Age, Technical University of Vienna, Austria, June 18, 2002

[3]: See 'IEEE 1588 Standard for a Precision Clock Synchronization Protocol for Networked Measurement and Control Systems' at http//ireel 588.nist.gov

[4]: "Draft Revision of IEEE Std 1139-1988. IEEE Standard Definitions of Plysical Puantites For Fundamental Frequency and Time Metrology-Random Instabilities." E.S. Ferre-Bikal, J.R. Vig, J.C. Camparo. L.S. Culer, L. Malek, W.J. Riley, S.R. Stein. C. Thomas, F. W. Walls and 1.D. White, 1997 IEEE Intemational Frequency Control Symposium Procedings.

[5]: See htp//wrw eecis.udeledu/ ntp/ for details concerning the Network Time Protocol.

MASTER

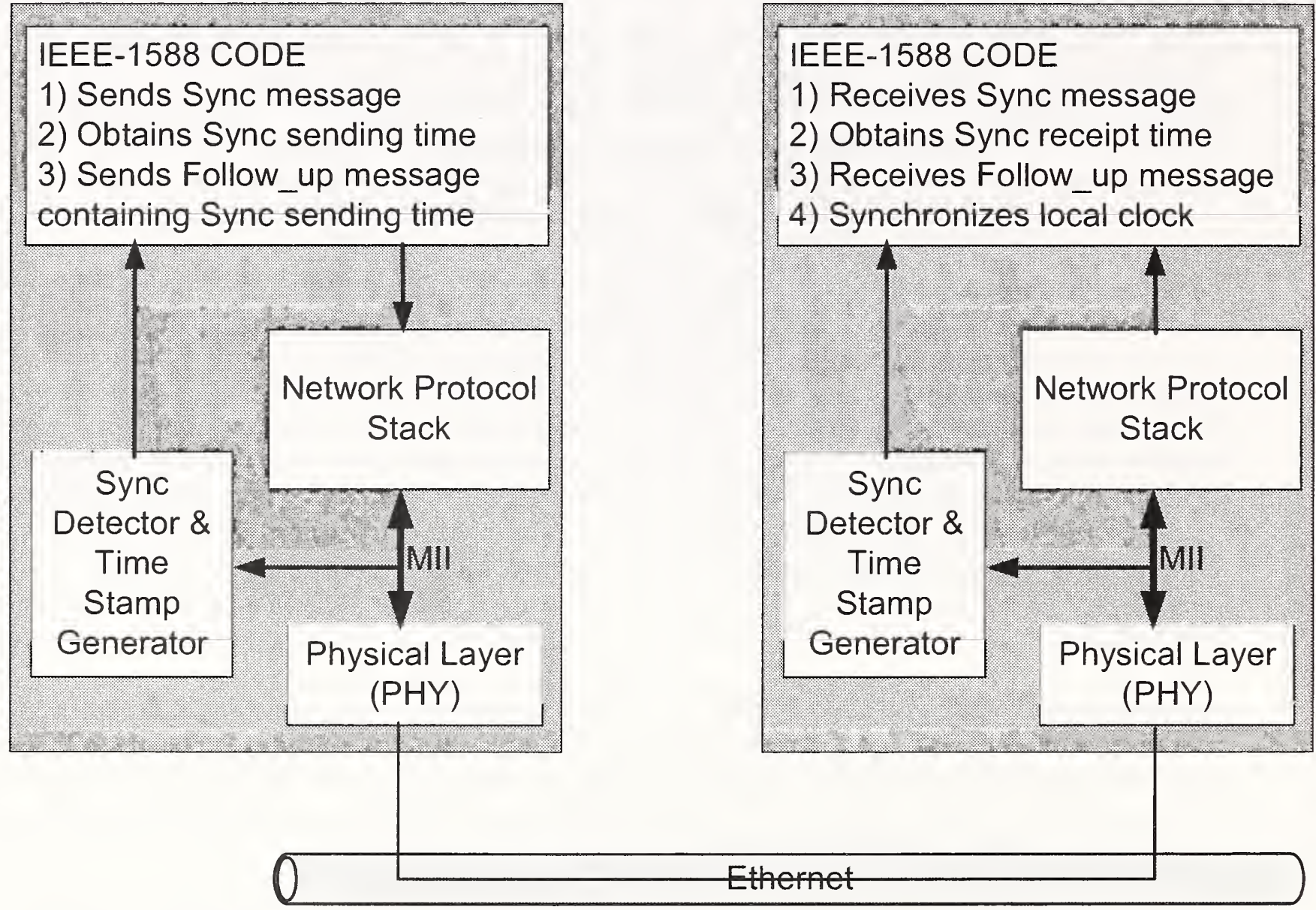

Figure 1: IEEE-1588 messages used in high accuracy synchronization 
MASTER

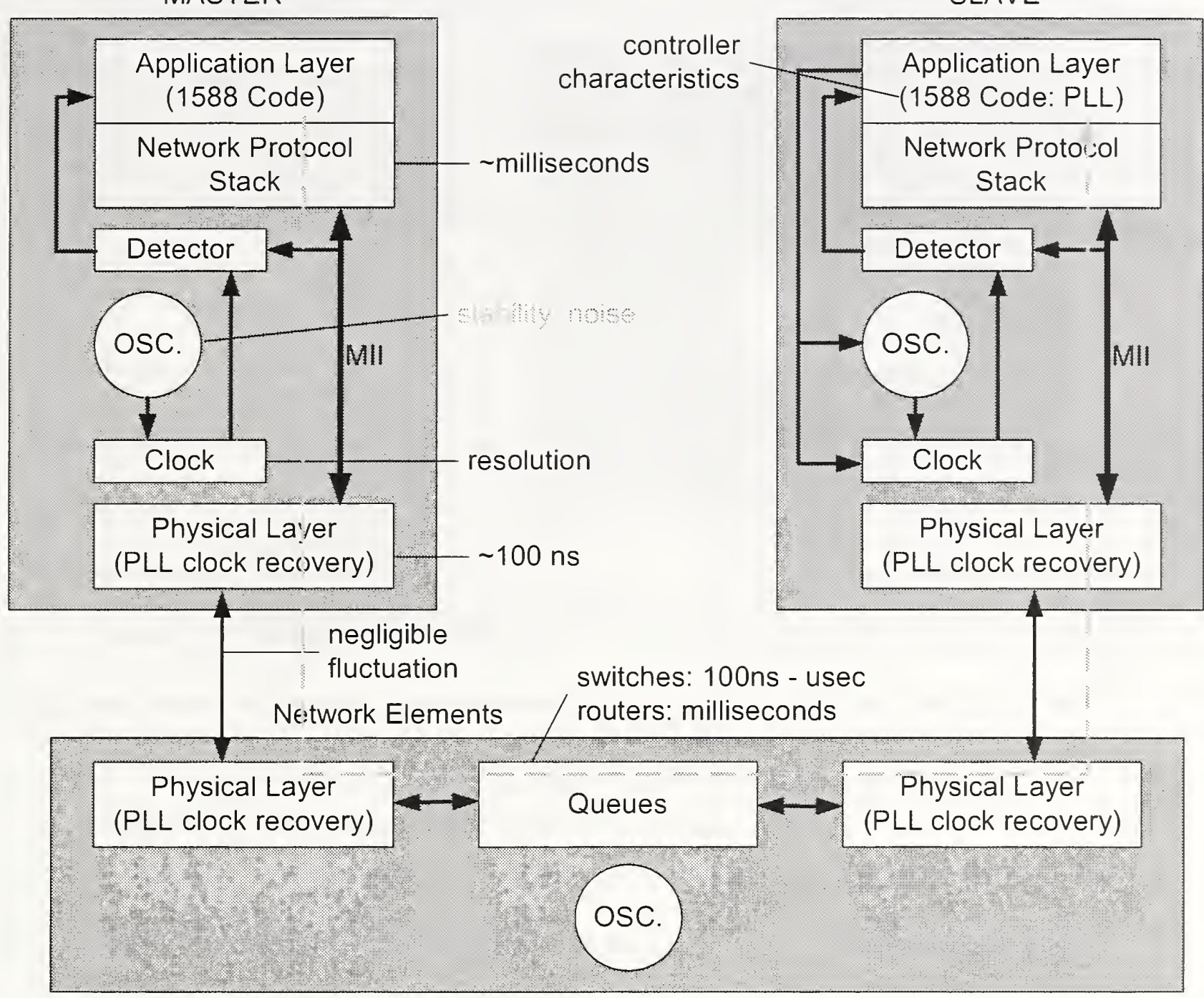

Figure 2: Sources of timing fluctuation in an IEEE 1588 system

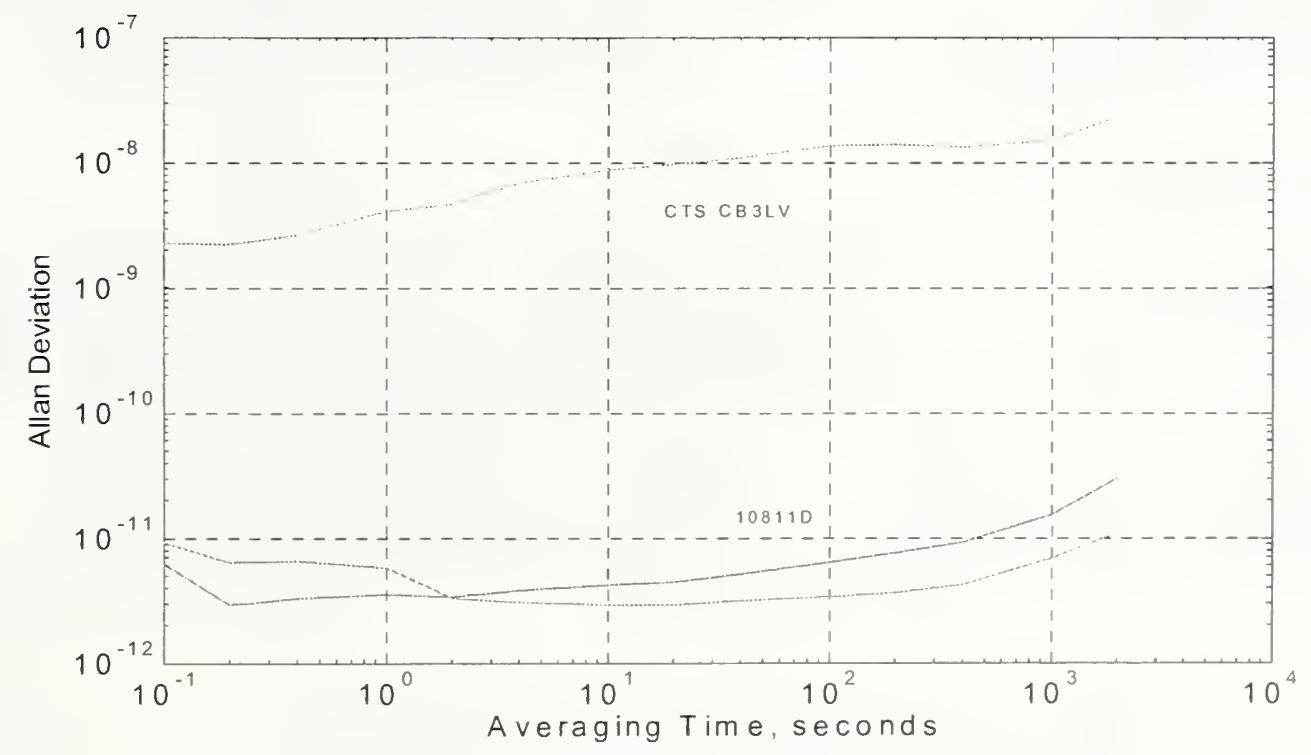

Figure 3: Allan frequency deviations for test oscillators 


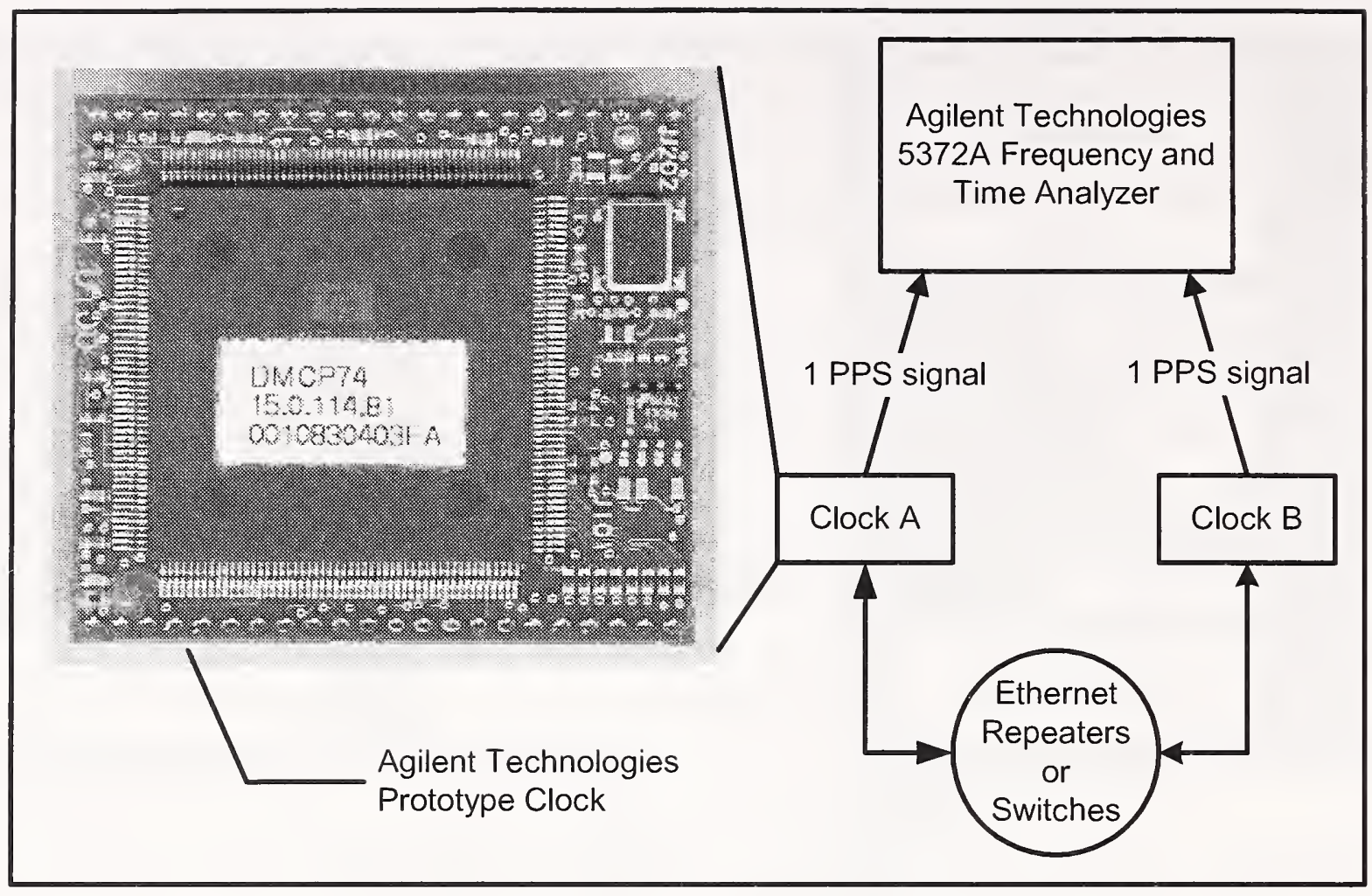

Figure 4: Measurement topology

$\bigcirc-\mid$ IEEE 1588 clock, code \& hardware

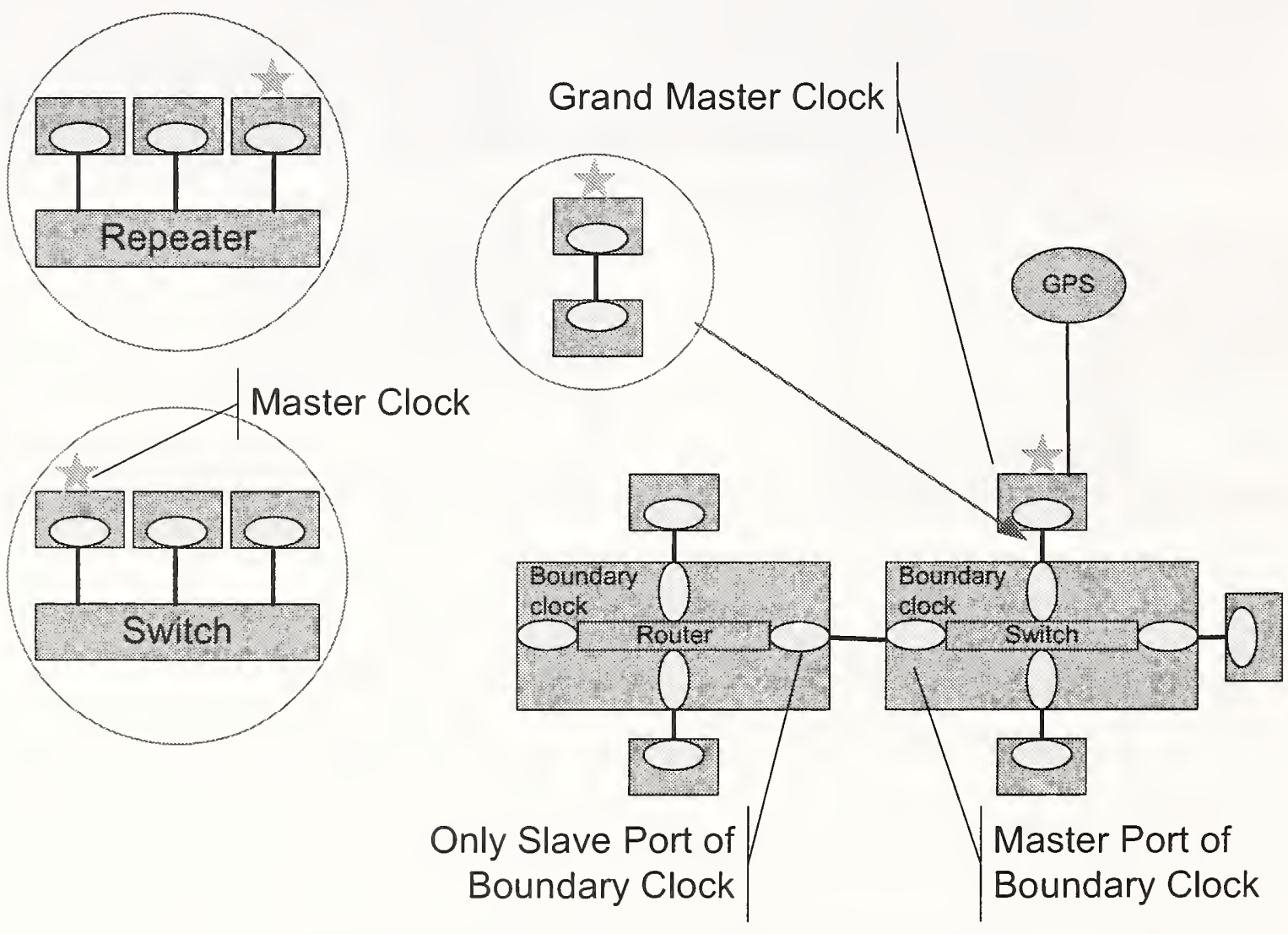

Figure 5: Experimental network connection topologies 

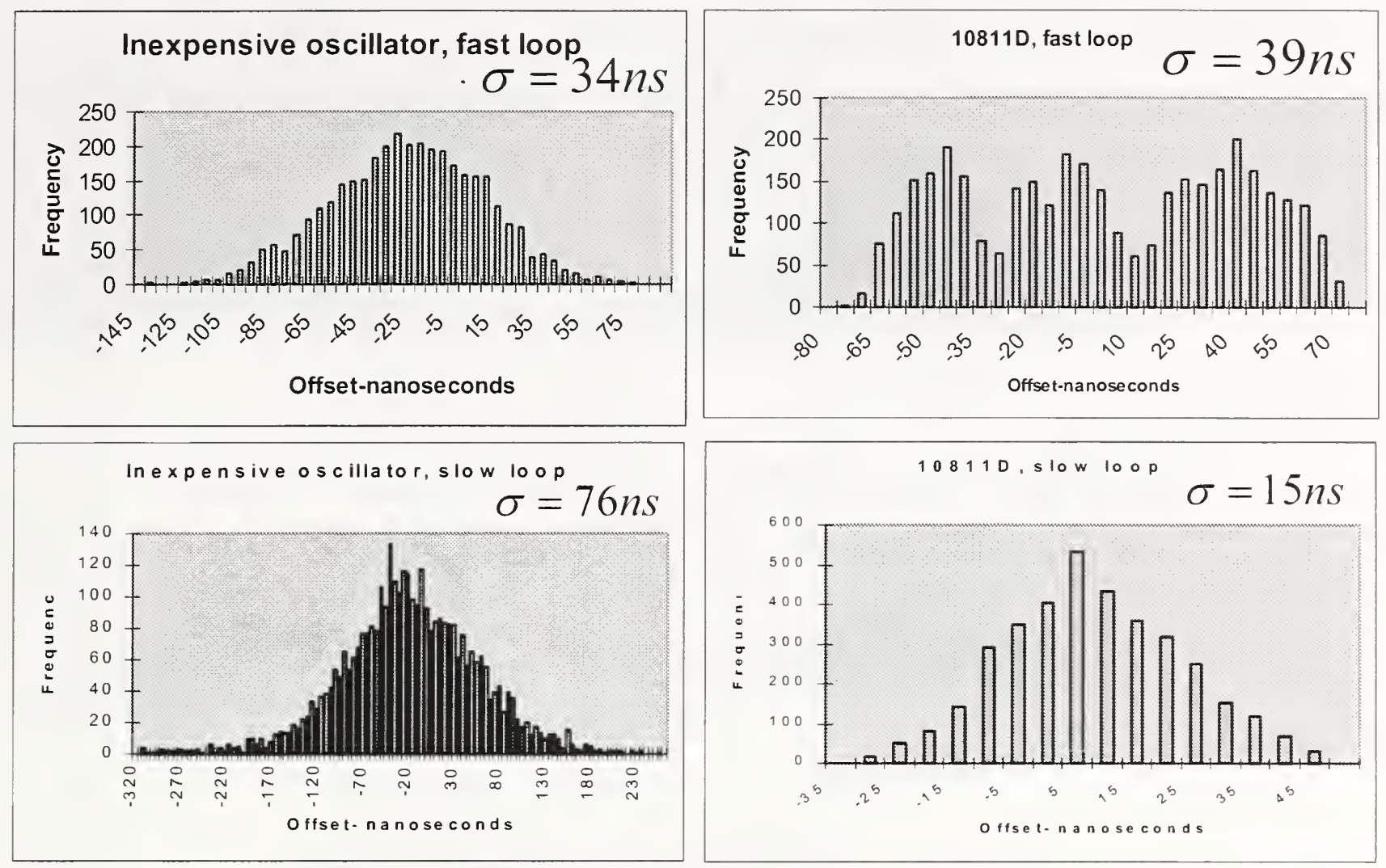

Figure 6: Histogram of synchronization error for direct connection
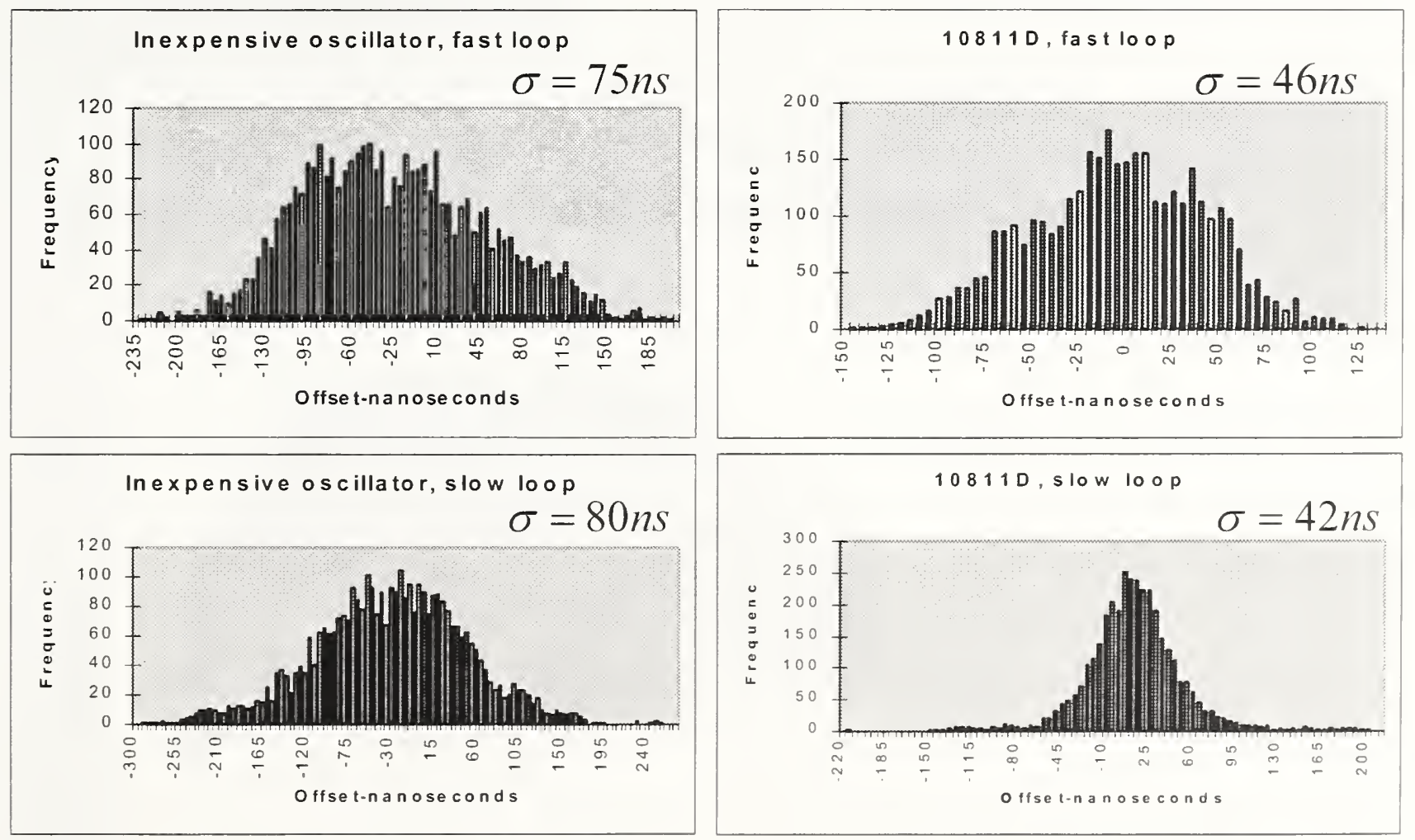

Figure 7: Synchronization error for connection via a repeater 

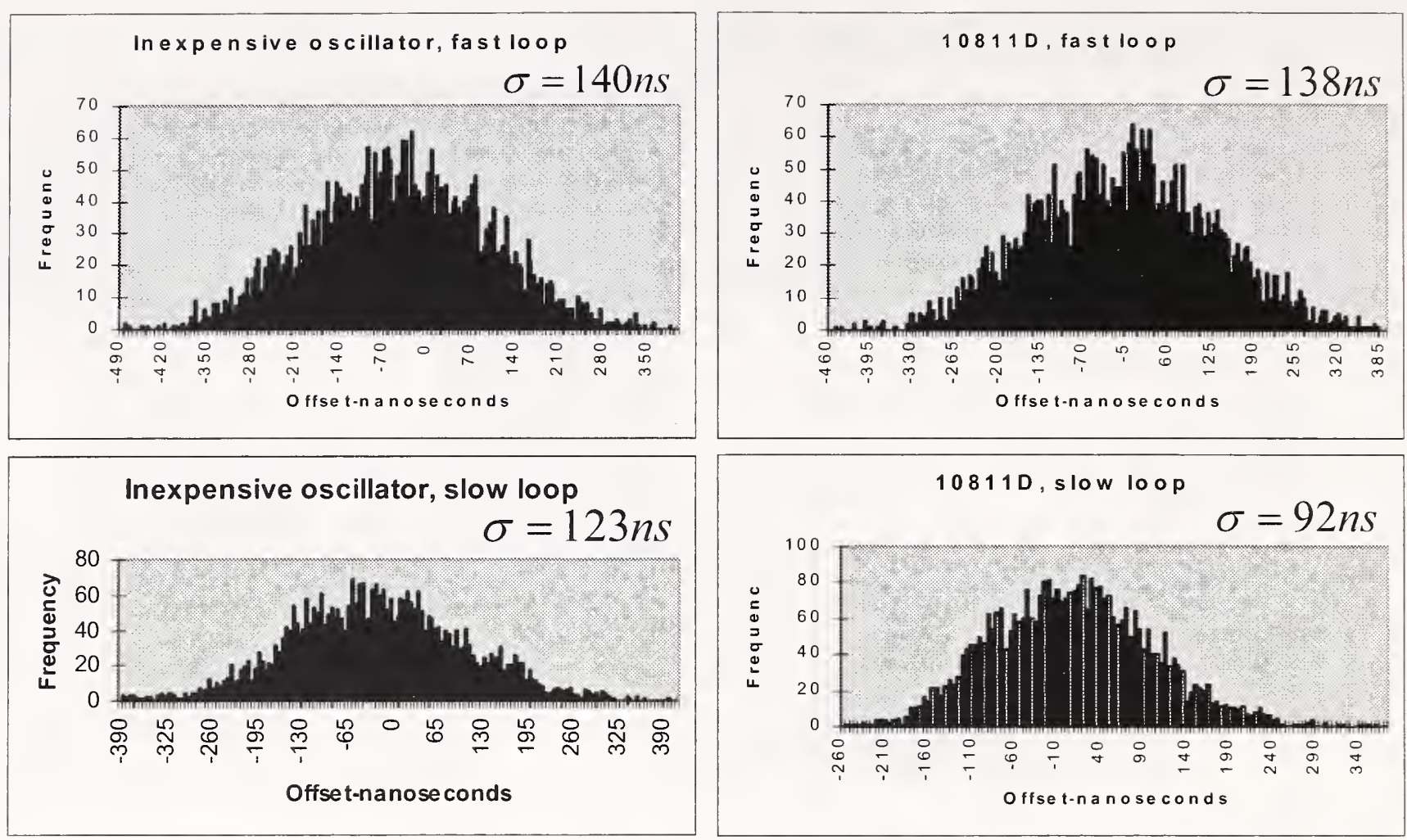

Figure 8: Synchronization error for connection via a switch 


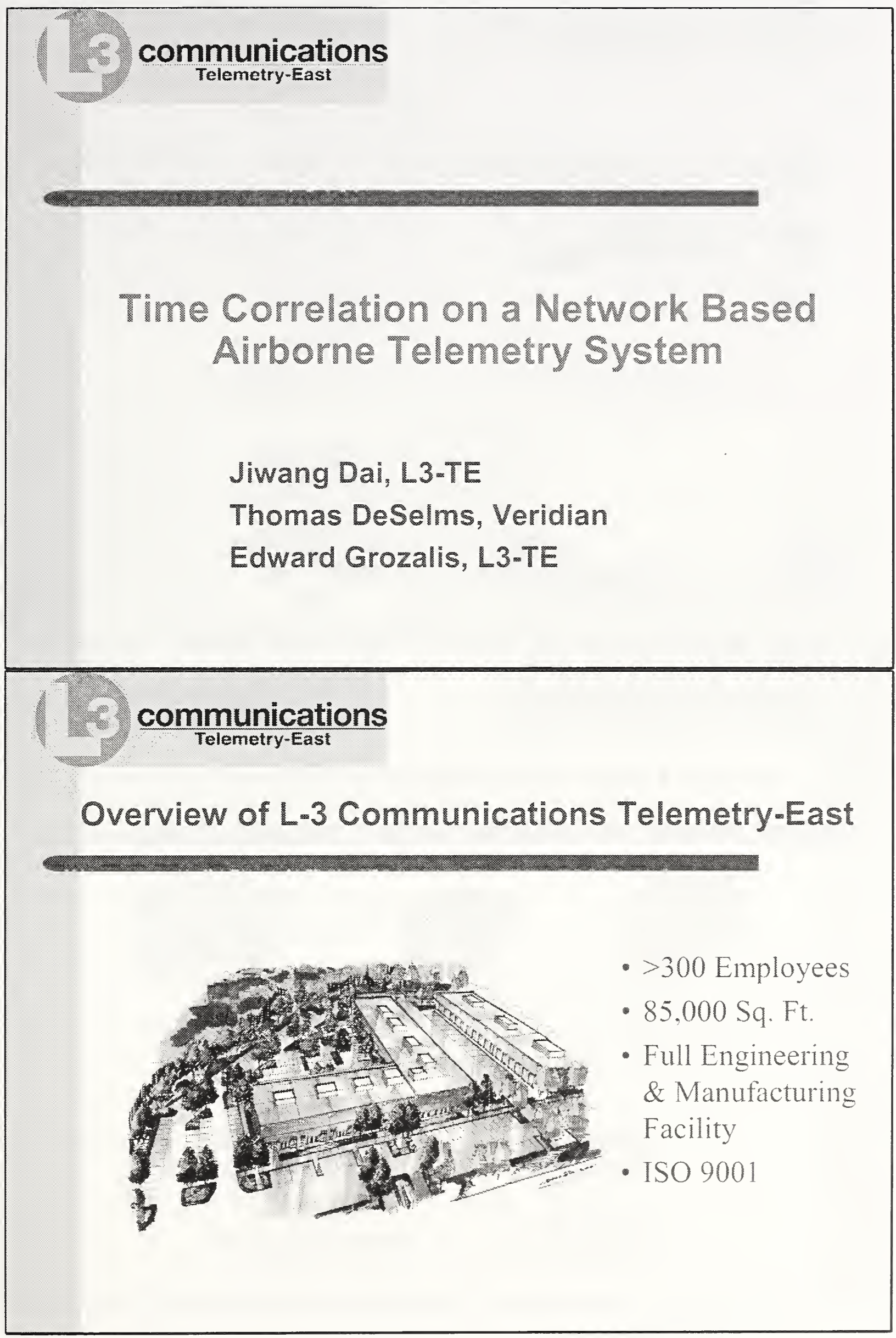




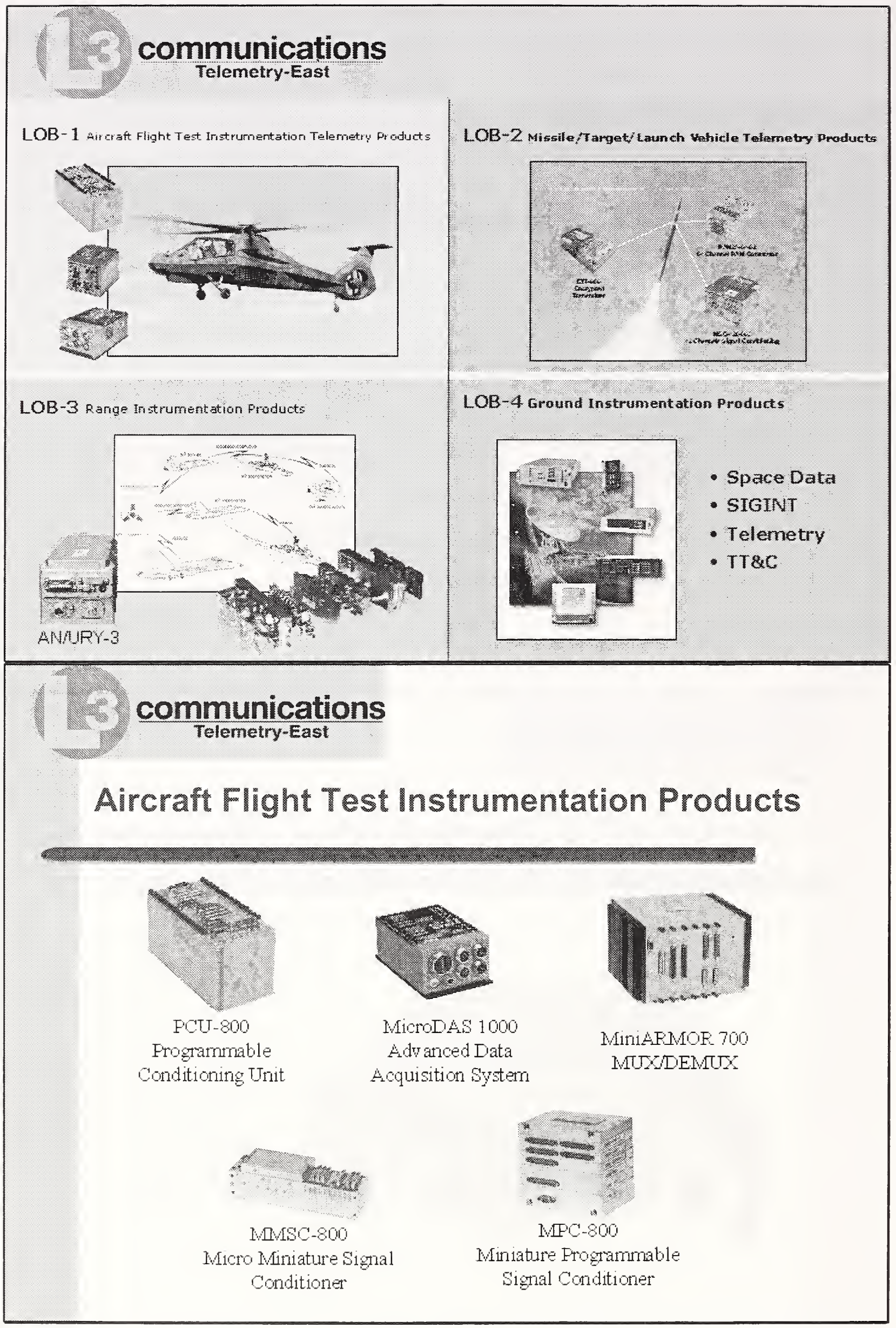




\section{communications}

Telemetry-East

\section{Legacy Telemetry System (standalone)}

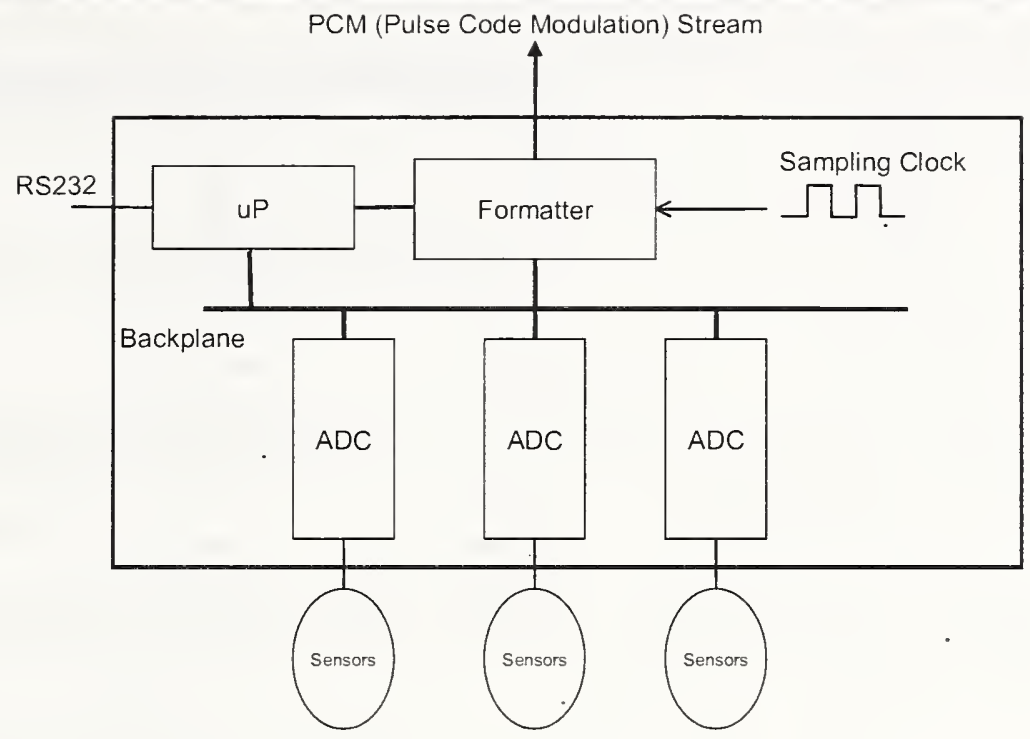

\section{communications}

Telemetry-East

\section{Legacy Telemery System (WasterRemote Slave)}
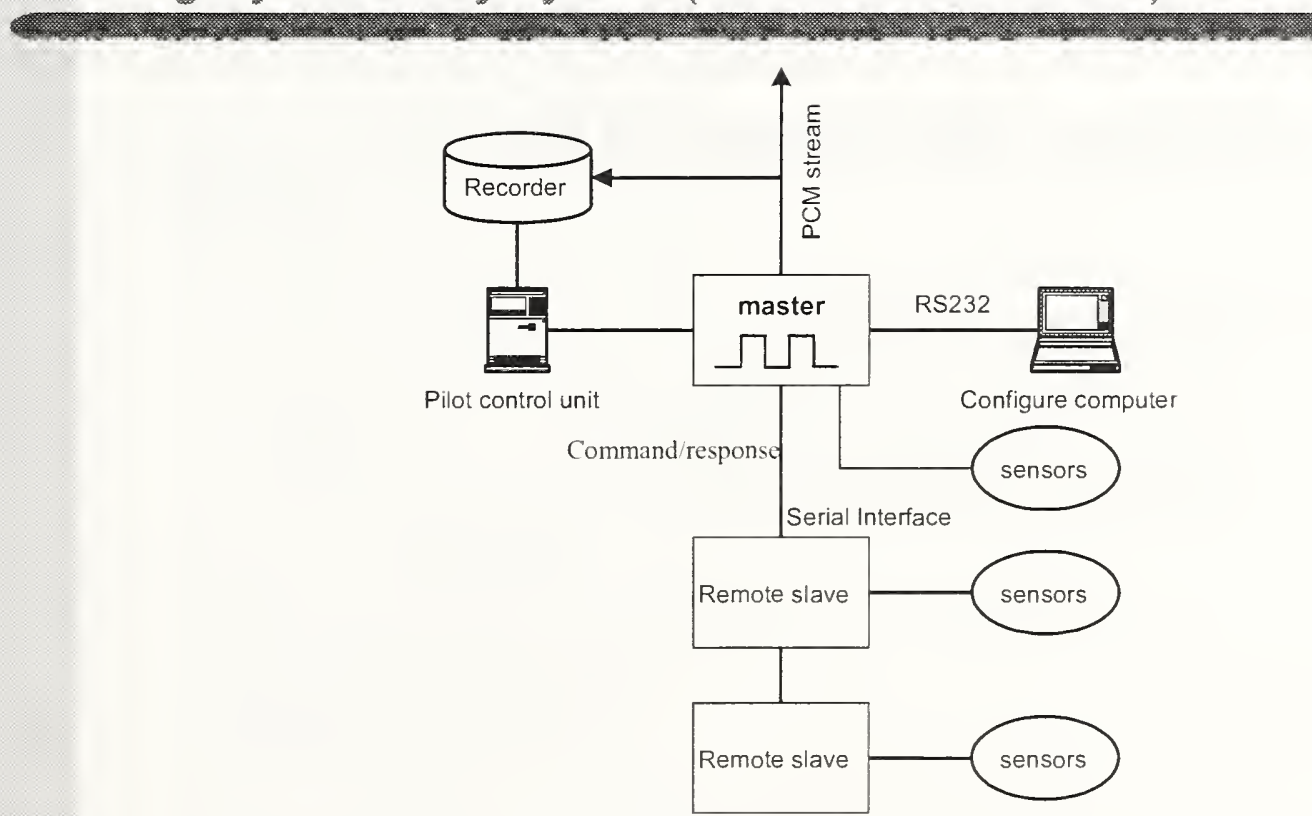


\section{communications}

Telemetry-East

Advanced Legacy Telemetry System (Multiple Masters)
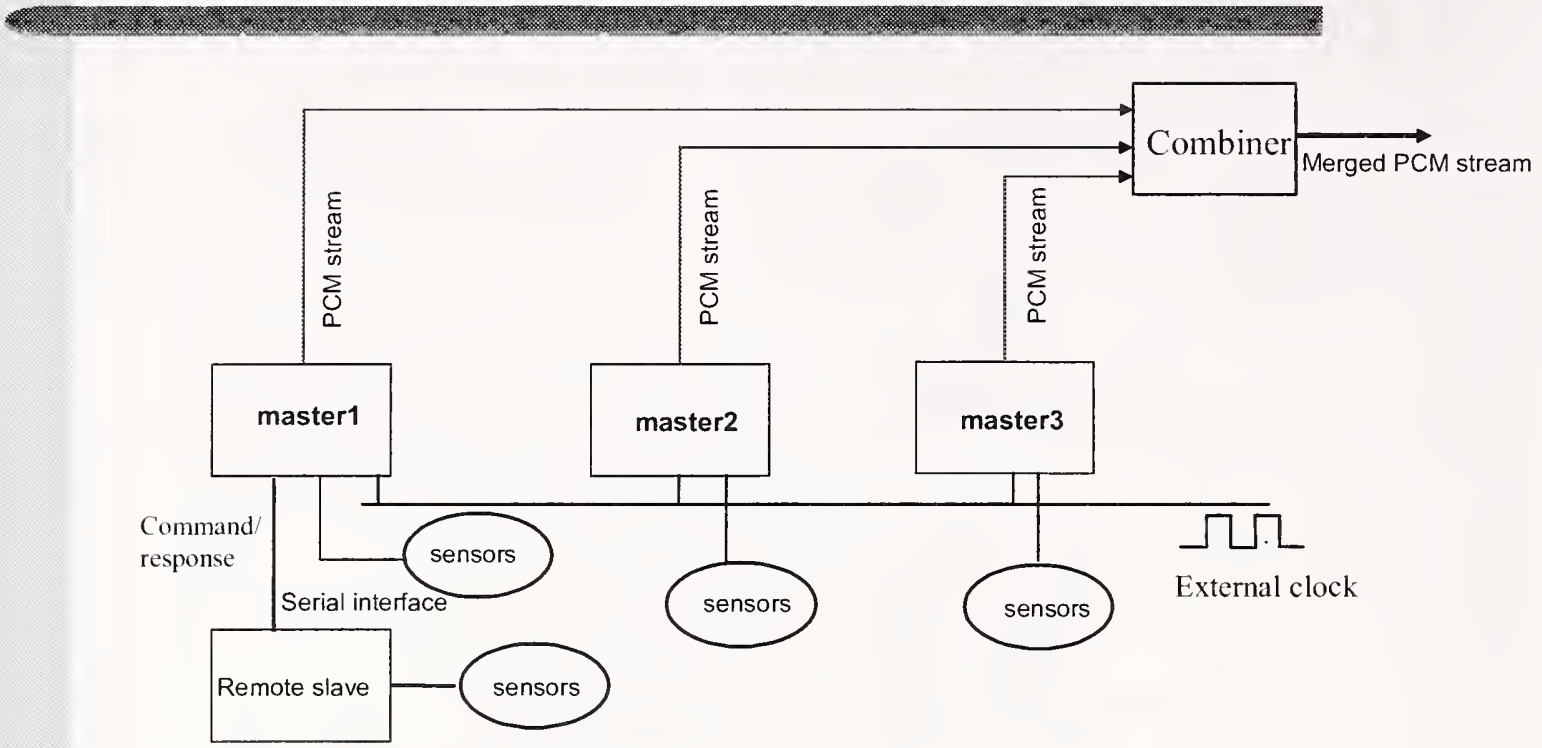

\section{communications}

$$
\text { Telemetry-East }
$$

Limization of the Legacy System

- Relatively low bit rate ( $\sim 5 \mathrm{Mbps})$

- Proprietary Interface

- Limited Interoperability

- Limited Usage of COTS 


\section{communications}

Telemetry-East

Network Based Telemetry System

- Better Interoperability

- Improved Scalability

- Higher Bit Rate

- Better Usage of COTS

\section{communications}

Telemetry-East

(1)

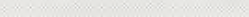

Architecture of the Network Based Telemetry System

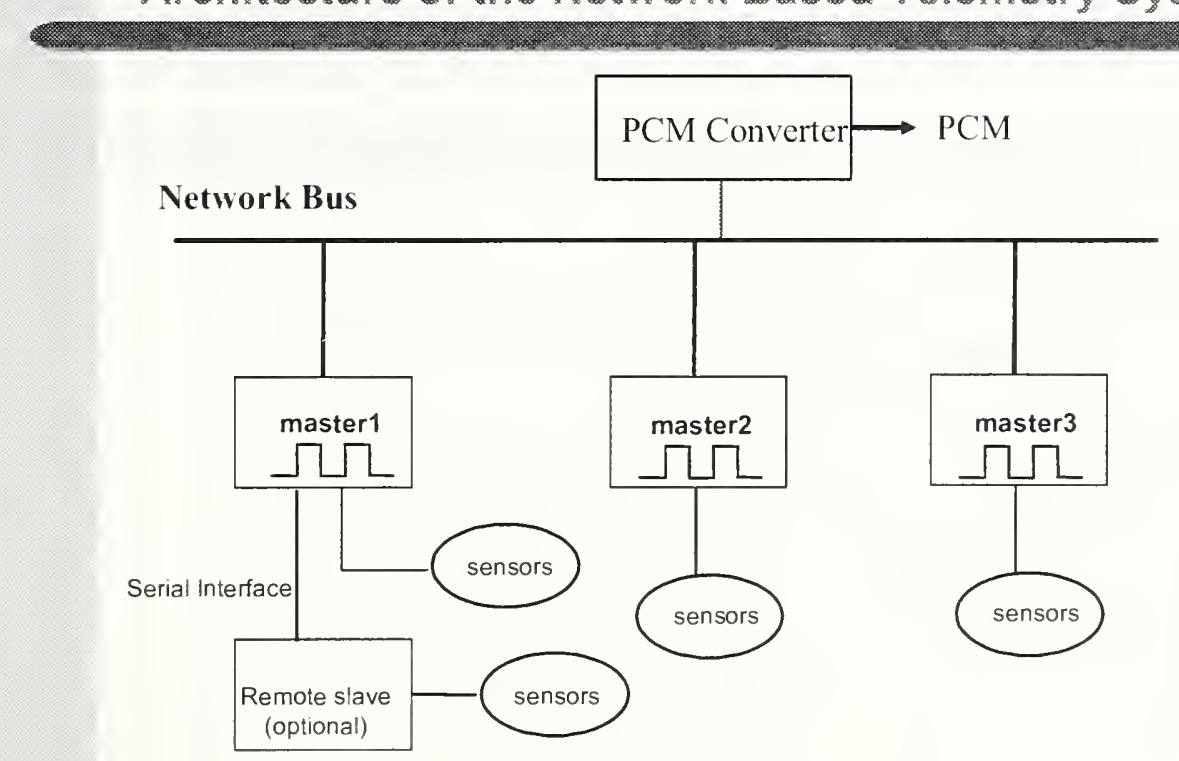




\section{communications}

Telemetry-East

How to Synchronize the clock of Masters?

- Add Additional Interface for Clock Sync

- Proprietary Solution (e.g. External clock)

- IRIG Time

- Use the Network Bus for Clock Sync

\section{communications \\ Telemetry-East}

\section{Choices of the System Bus}

- Fibre Channel Avionics Environment (FC-AE)

- Embedded Time Sync Design

- Expensive

- Lack of Industrial Switches

- Ethernet

- Cost Saving

- Mature Technology

- No Time Sync

- Others 


\section{communications}

Telemetry-East

Time Correlation Methods for the Ethemet

- $\operatorname{NTP}(10$ 's $\mu \mathrm{s})$

- $\operatorname{PTP}(<1 \mu s)$

(Ref. IRIG-G, 20ns )

\section{communications}

Telemetry-East

Time Sync Requirements

- 1K Signal

- 12 Bits accuracy : 39 ns

- 10 Bits accuracy : 155 ns 


\section{communications}

Telemetry-East

\section{A NPCU.800 Unit}

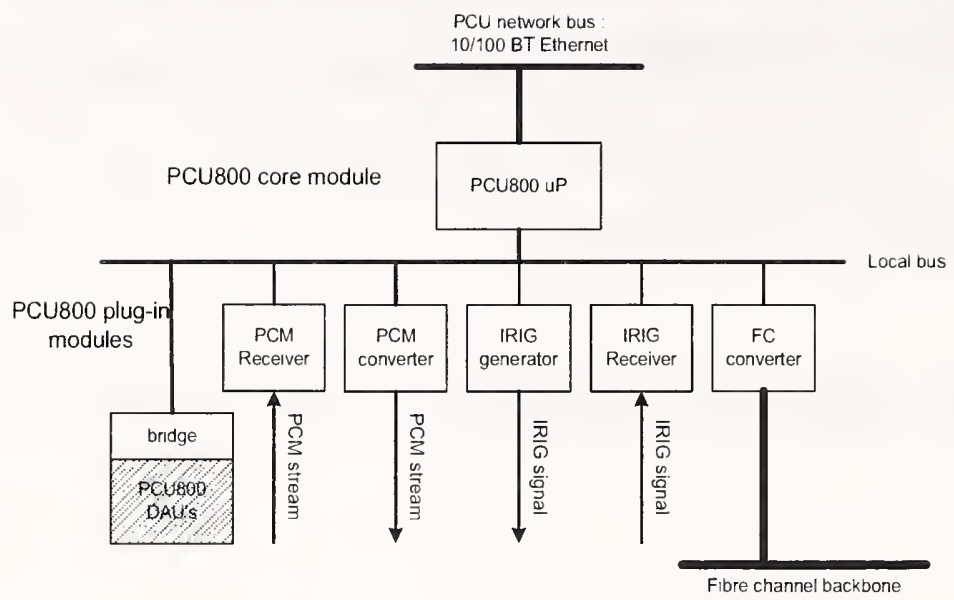

\section{communications}

\section{Telemetry-East}

\section{PTP Hardware Implementation}

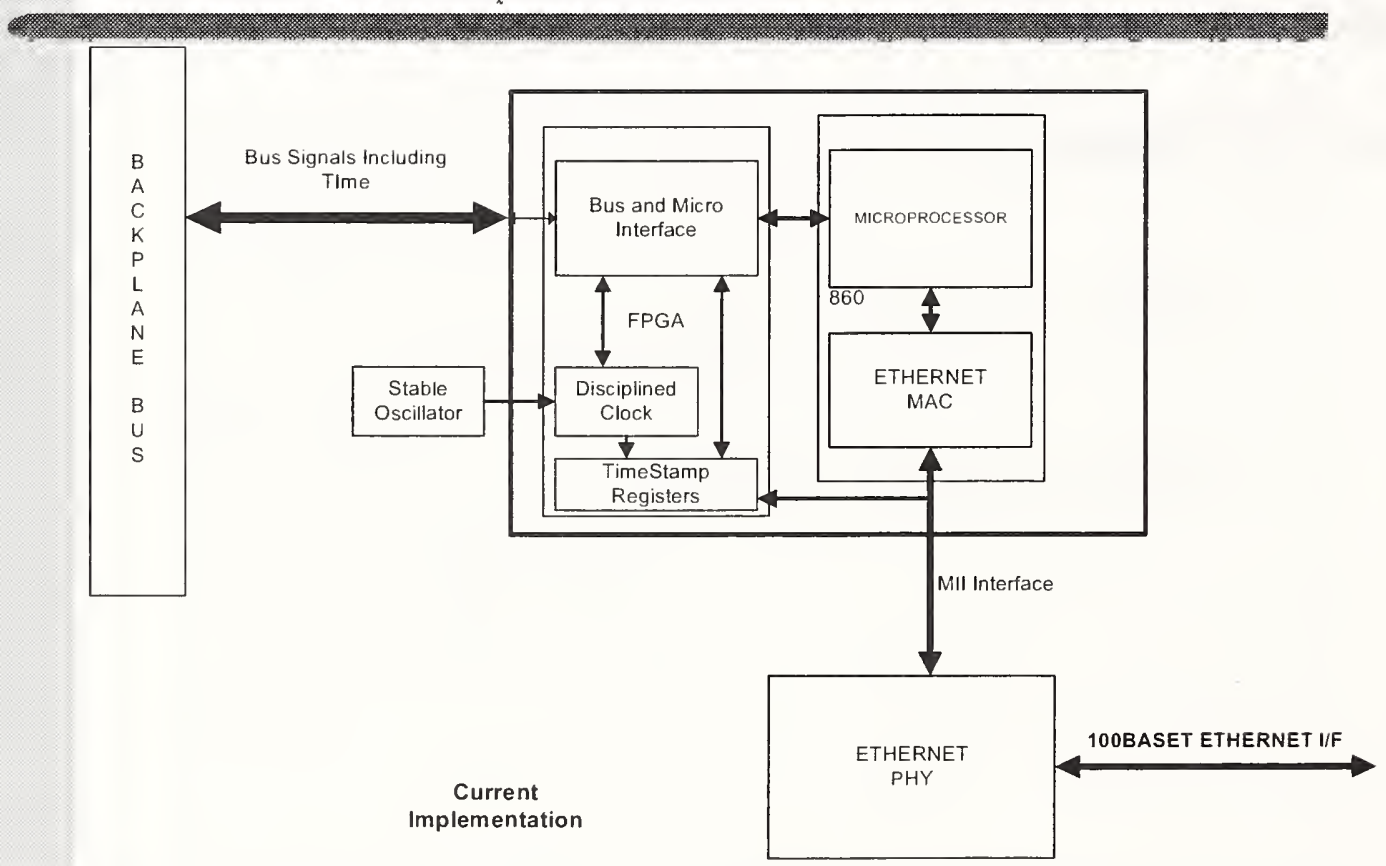




\section{communications}

Telemetry-East

Implementation Details

- Stable Oscillator - a Temperature Compensated Crystal Oscillator(TXCO)

- FPGA - The "glue" logic of the system

- Timestamp registers

- Control logic to discipline the clock

- Bus interfaces to the microprocessor and the Backplane Bus.

- Microprocessor - A Motorola 860 PowerPC (Internal Ethernet MAC)

- Ethernet PHY

\section{communications}

Telemetry-East

\section{Ehernet Based Telemery System}

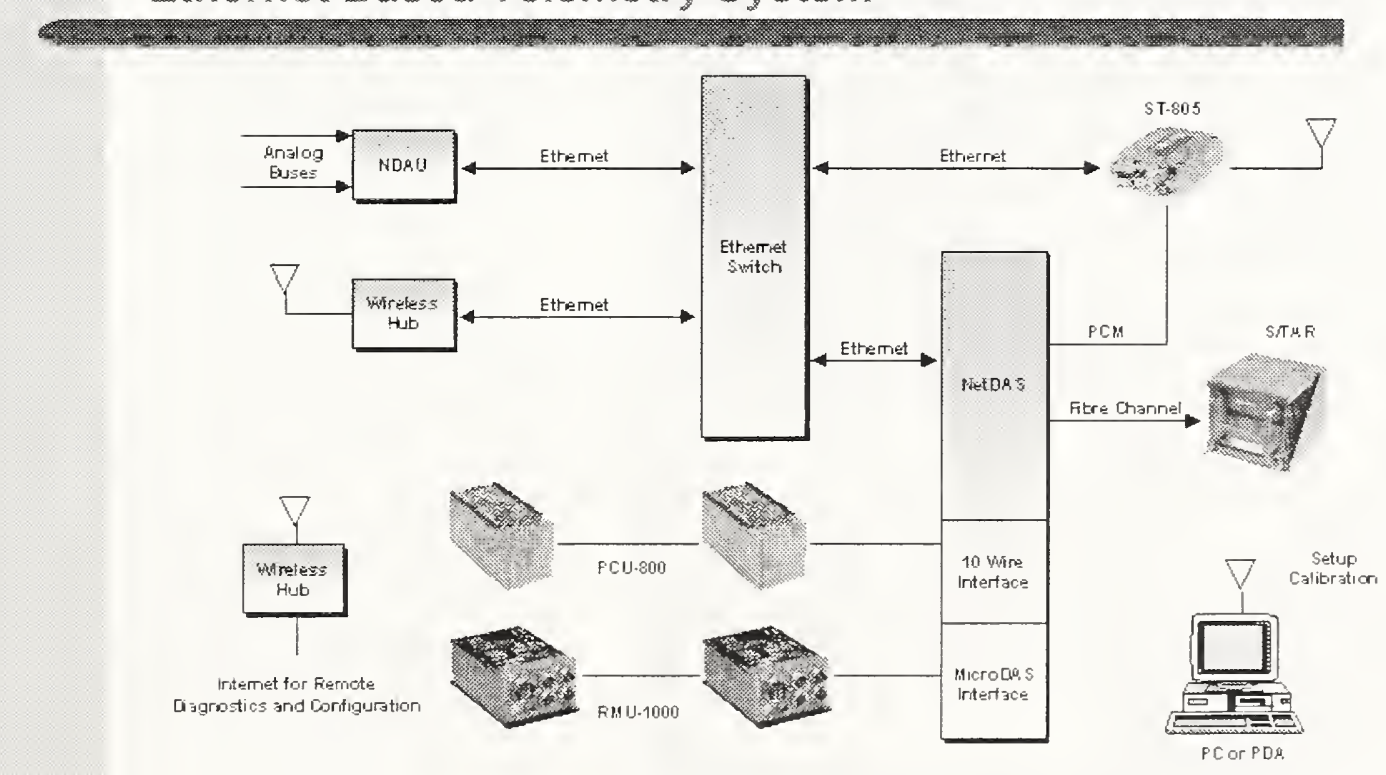


g

\title{
Implementation of IEEE Std.-1588 in a Networked I/O Node
}

\author{
Mark E. Shepard \\ Douglas G. Fowley \\ Roy L. Jackson \\ Dennis B. King
}

GE Drives \& Controls, Inc.

Salem, VA

g

- Turbine controls

- Large stationary gas turbines (10 - 200 MW)

- Aeroderivative turbines for marine, oil \& gas (25 - $75 \mathrm{MW}$ )

- Large steam turbines (20 - $1500 \mathrm{MW})$

- Hydroelectric turbines

- Power conversion products

- Turbine static starters

- Excitation products

- System drives

- Rolling mill main drives

- Marine propulsion

- Converters for alternate energy - wind, fuel cell, etc.

- Drive Systems - recently spun off to a JV with Toshiba 


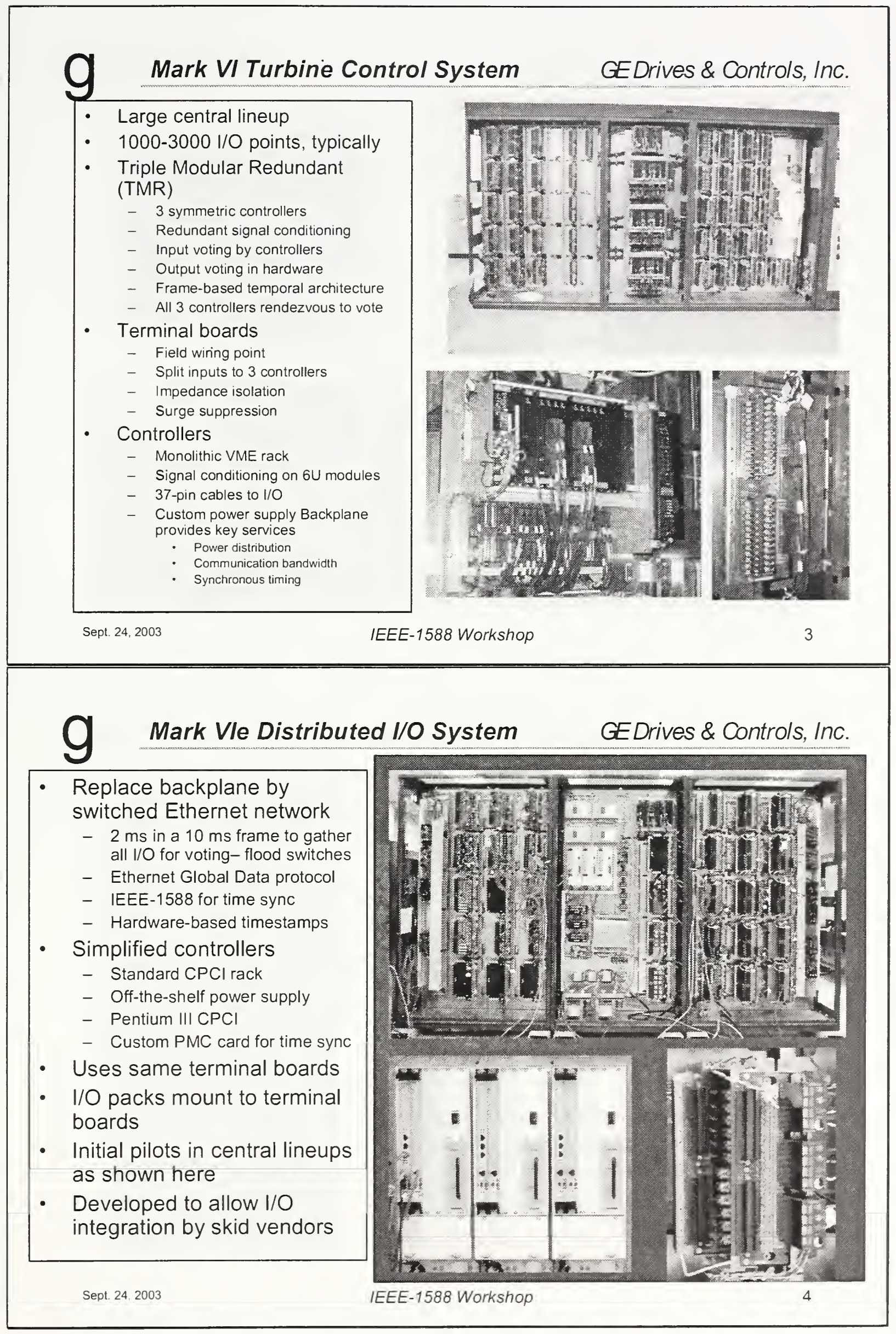


- CPU card common to all packs

- AMD Alchemy Au1000 SoC

- 266MHz MIPS32 CPU

- Dual 10/100 Ethernet, IrDA

- 100K Xilinx Spartan-II FPGA

- QNX Neutrino RTOS

- Specific signal-conditioning board for each $1 / O$ type

- Industrial-hardened

- $0-60 \mathrm{C}$ at full accuracy

- $-40-+70 \mathrm{C}$ operating

- Shock, vibration, EMI
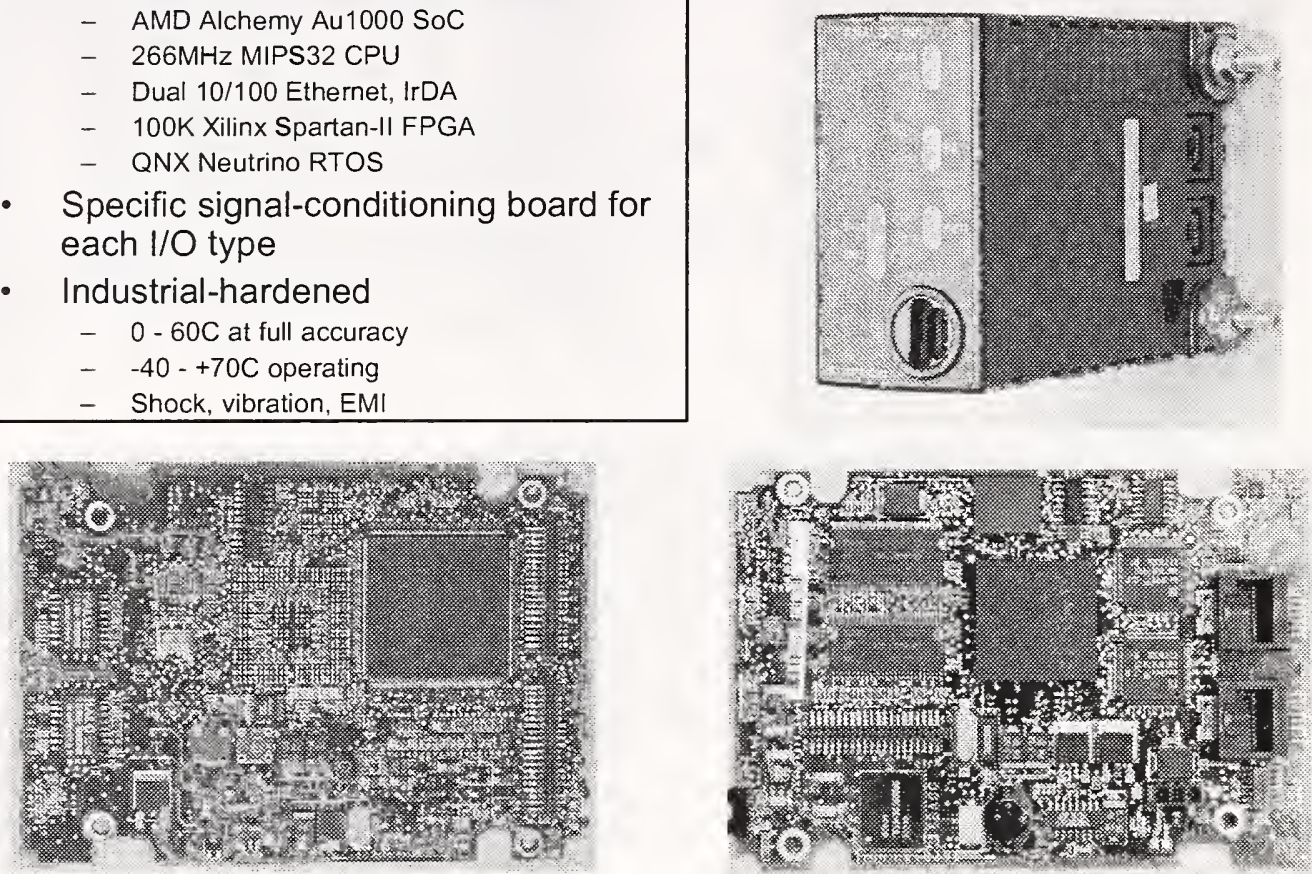

\section{PMC Ethernet with hardware-assist GEDrives \& Controls, Inc.}

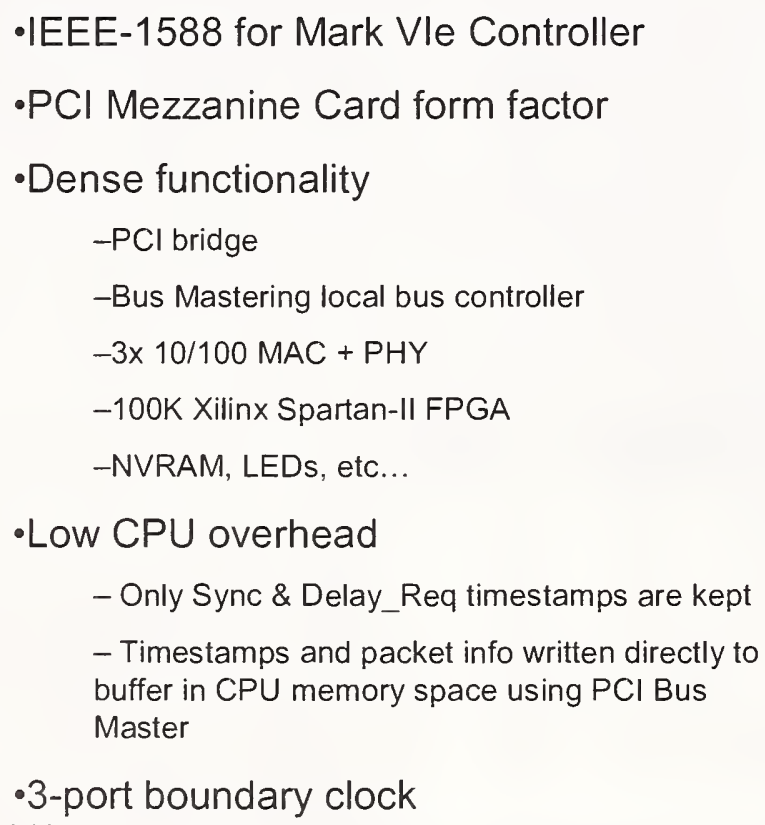

- Only Sync \& Delay_Req timestamps are kept - Timestamps and packet info written directly to buffer in CPU memory space using $\mathrm{PCI}$ Bus Master

-3-port boundary clock

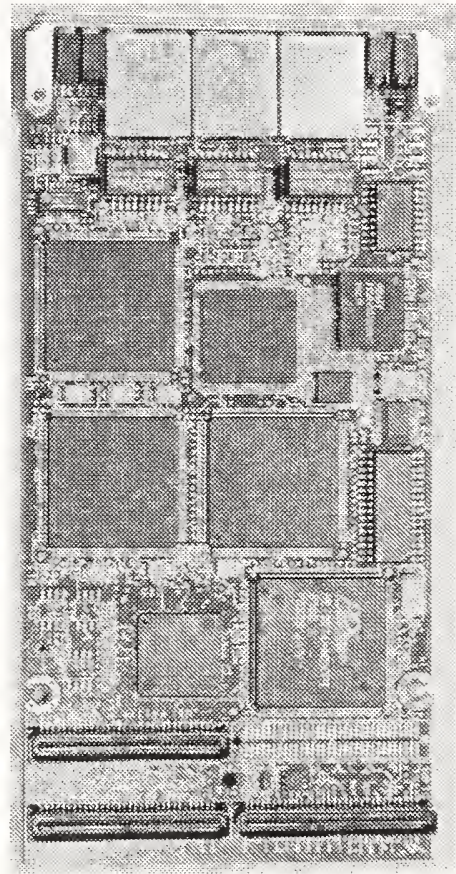


- In the Turbine control system

- UTC reference is important for timestamping events for "flight-data-recorder" usage

- But - turbine operation must never be compromised by a lost or corrupt UTC reference

- Networked nodes must remain synchronized to their controllers, ignoring ambiguous or conflicting UTC references (esp. from NTP)

- So we choose to avoid

- Arbitrary autonomous selection of their time master by network nodes

- Possible bidding for slaves by rogue masters

- Other unanticipated failure modes from the autonomy inherent in IEEE-1588

- The Mark Vie will apply IEEE-1588 so that:

- The nodes and controllers are synchronized to a time reference with arbitrary epoch which we call System Time, using ClockIdentifier INIT

- Only the controller which is the System Time GrandMaster is empowered to coordinate the Mark Vle internal time reference with UTC

- The epoch of System Time, expressed as UTC, must be maintained outside the PTP protocol

- The system can run without GPS/UTC

- All nodes belong to CommunicationID PTP_CLOSED

- Each controller R, S, T uses a distinct AlternatePTPDomain for its nodes

- The three controllers belong to DefaultPTPDomain

- The controllers maintain separate synchronization to UTC sources with additional virtual clocks in the DefaultPTPDomain on CommunicationID PTP_ETHER

\section{Need better-defined accommodation for this in IEEE- $1588 ?$}

- 64-bit register (adder) with time in ns

- Locked to remote clock by PLL

- Clocked by local oscillator

- VCO - increment by clock period (in ns)

- Latch register time at SFD on PHY

- Error detector and regulator can be closed in $\mathrm{h} / \mathrm{w}$ or $\mathrm{s} / \mathrm{w}$

- Adjust VCO by $0, \pm 1$ increment value

- Pros

- Can be self-contained

- All nodes have synchronized registers

- Cons

- Fairly complex logic

- Replicated for each clock

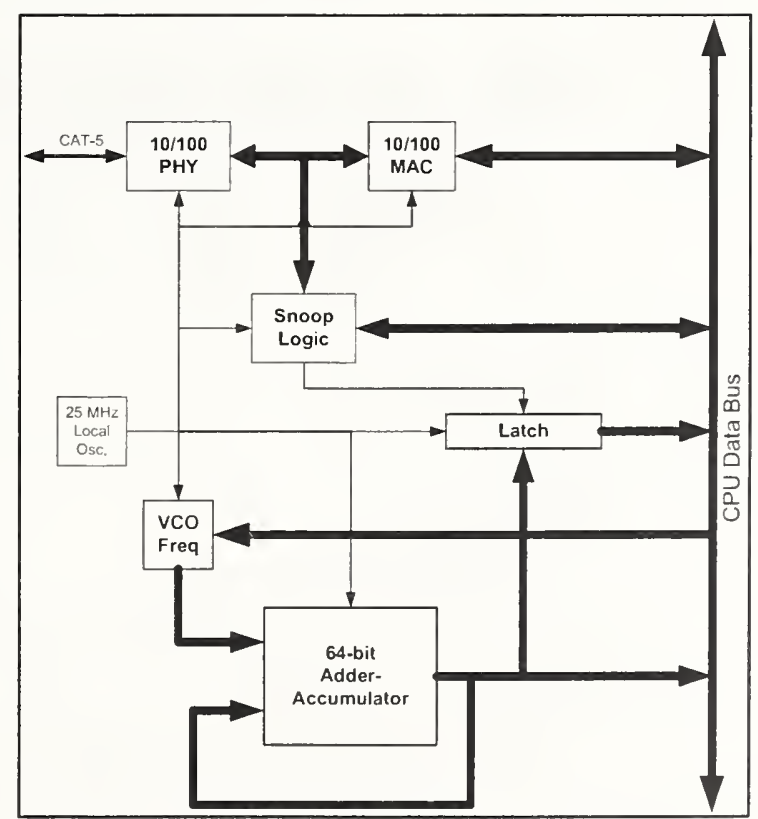




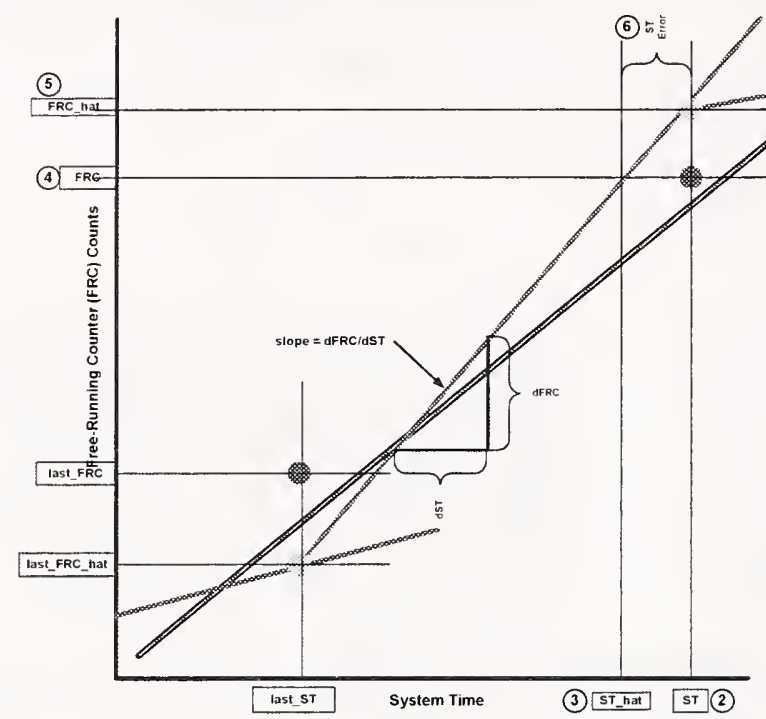

(7) new_slope

- Hardware free-running counter (FRC) latched for incoming timestamp

- Piecewise-linear model of FRC vs. System Time

- Slope (VCO frequency) constant between synchronization points

- Error calculated from timestamps in Sync messages

- Slope is output of PI regulator looking at error

- Software functions convert between FRC and System Time

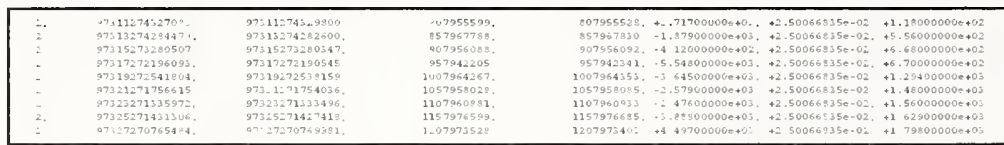

- Data written to text file on RAMdisk in node

- Use FTP to download, Matlab to analyze and plot

- Show raw timestamp data and PLL tracking

- Detrend and magnify

- Scale counts to usec
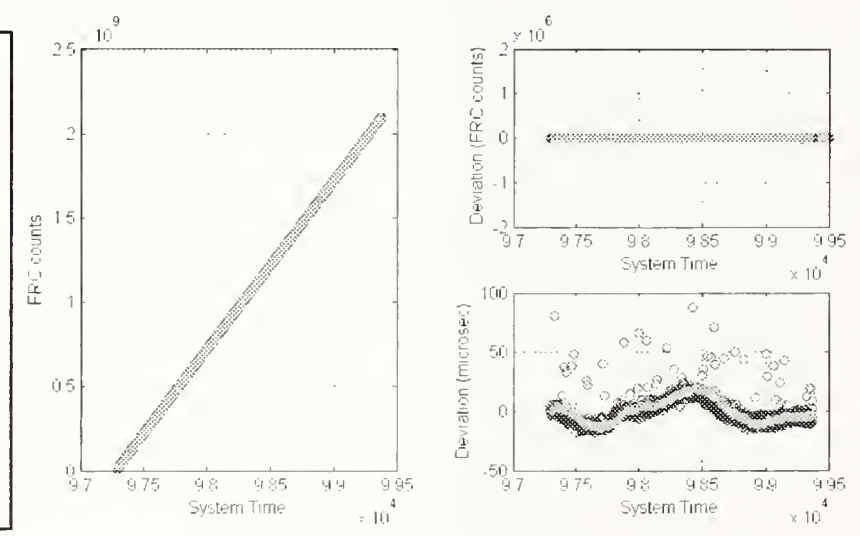


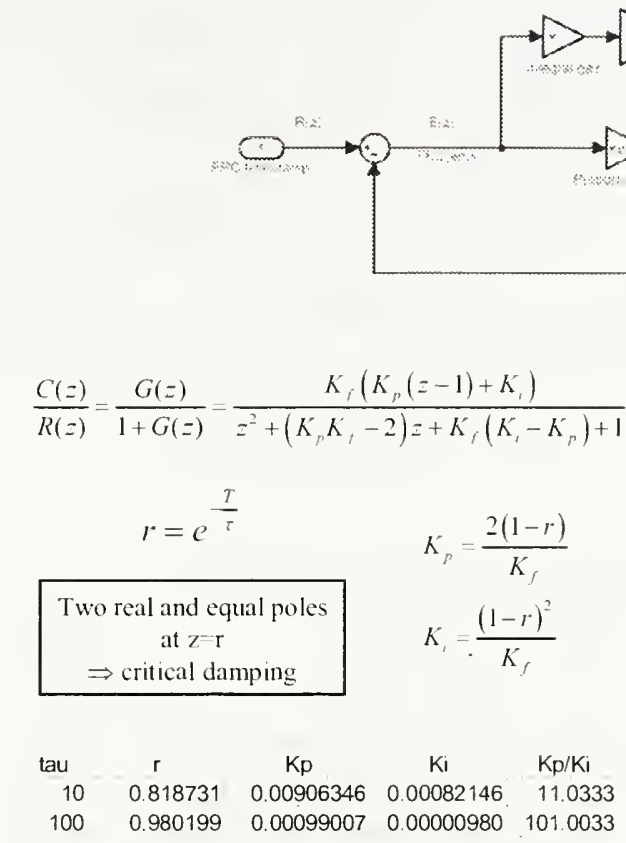

\section{PLL Acquisition}

GEDrives \& Controls, Inc.

- Use 10 Sync messages

- Linear regression, least squares fit

- Initialize integrator and first sync point

$$
\begin{gathered}
y=m x+b \\
m=\frac{\sum_{i} x_{i} y_{i}-\frac{\left(\sum_{i} x_{i}\right)\left(\sum_{i} y_{i}\right)}{i}}{\sum_{i} x^{2}-\frac{\left(\sum_{i} x_{i}\right)^{2}}{i}} \\
b=\left(\frac{\sum_{i} y_{i}}{i}\right)-m\left(\frac{\sum_{i} x_{i}}{i}\right)
\end{gathered}
$$

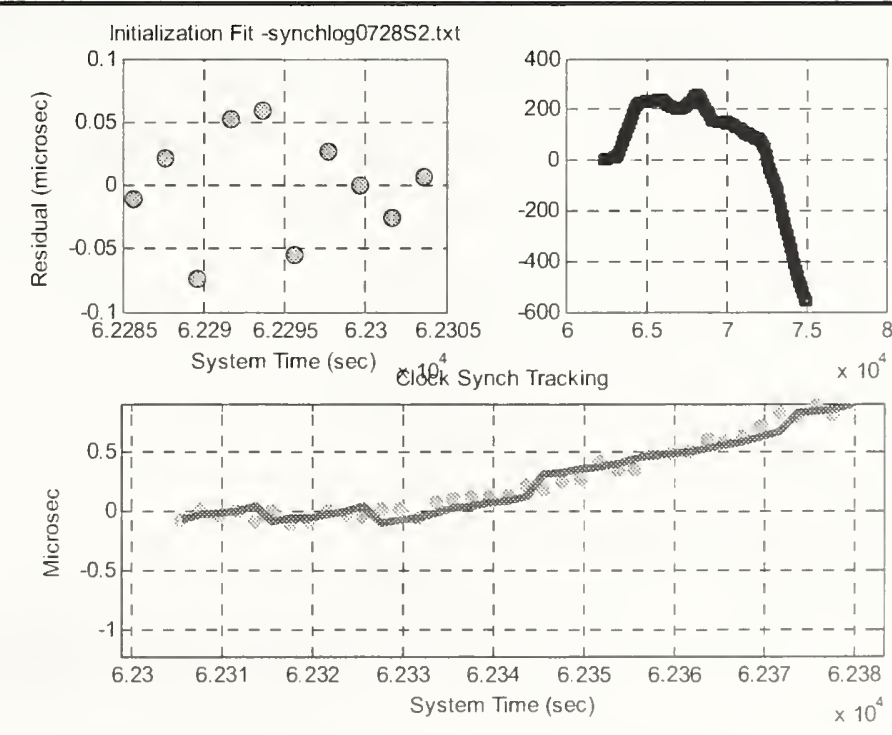


- Acquire both Sync and Delay_Req messages

- Fit regression model that uses both, constrains equal slopes

- Least-squares fit using SVD for matrix inversion

- Sort by residual value

- Apply Chauvenet's Criterion to largest residual. If largest residual is an outlier, discard and repeat

- Chauvenet's criterion: "You may consider rejecting the data when the number of measurements expected this far from the mean is less than one half " (assuming a normal distribution)

$$
\begin{gathered}
\left(\begin{array}{ccc}
S T_{1} & 1 & 0 \\
S T_{2} & 0 & 1 \\
\vdots & \vdots & \vdots \\
S T_{N} & 1 & 0
\end{array}\right)\left(\begin{array}{l}
m \\
b_{1} \\
b_{2}
\end{array}\right)=\left(\begin{array}{c}
F R C_{1} \\
F R C_{2} \\
\vdots \\
F R C_{N}
\end{array}\right) \\
\mathbf{A x}=\mathbf{b}
\end{gathered}
$$

$$
\begin{aligned}
(\mathbf{A}) \cdot \mathbf{x} & =\left(\mathbf{U} \cdot \mathbf{W} \cdot \mathbf{V}^{\mathrm{T}}\right) \cdot \mathbf{x}=\mathbf{b} \\
\mathbf{x} & =\mathbf{V} \cdot \mathbf{W}^{-1} \cdot \mathbf{U}^{\mathrm{T}} \cdot \mathbf{b}
\end{aligned}
$$

\section{Approximation of CDF}

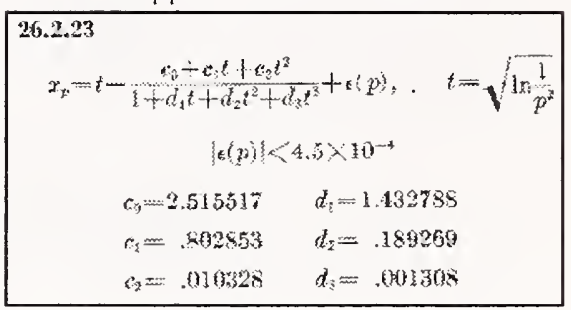

- 100 BaseTx Ethernet

- 3 switches in series

- $2.1 \pm 0.1$ us switch latency

- $10 \%$ probability of queuing delay in each switch

- Uniform queuing latency distribution, zero-max

- 1 Sync message/sec

- Random distribution of Delay_Req messages per IEEE-1588

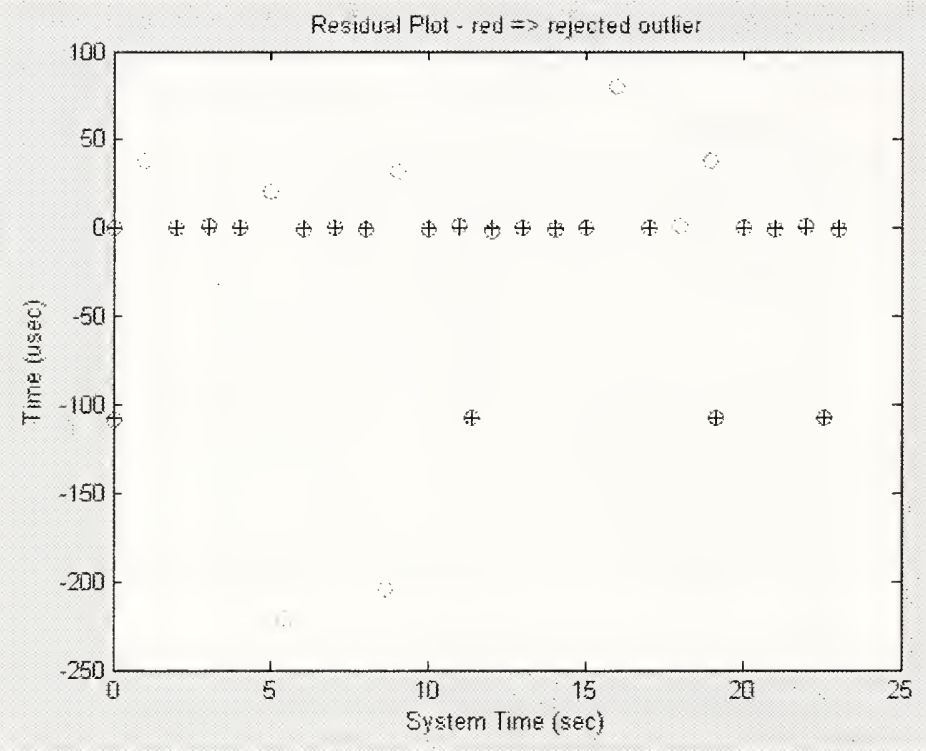


g
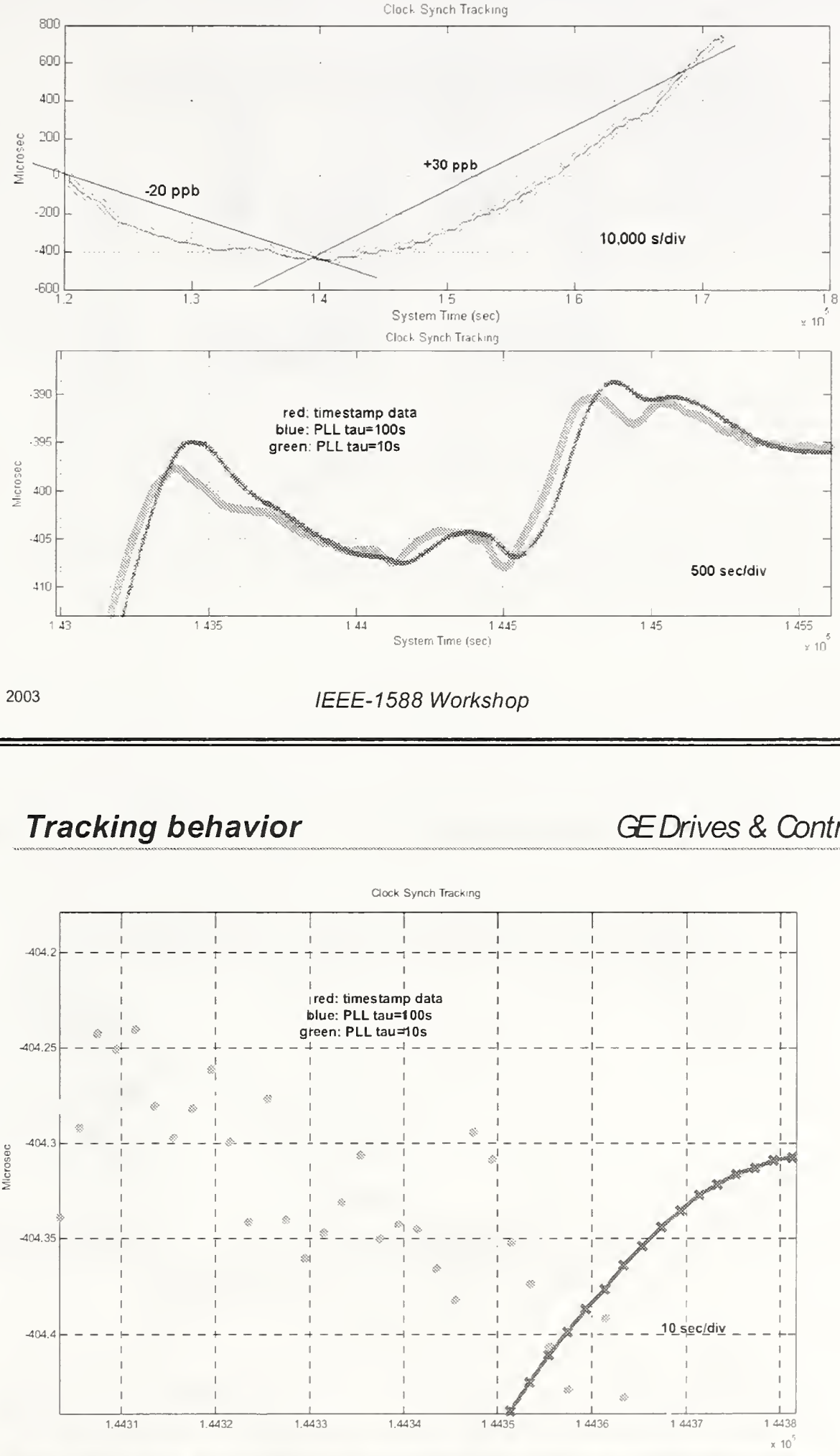

System Time (sec)

\section{It's all about the error estimator...}


g

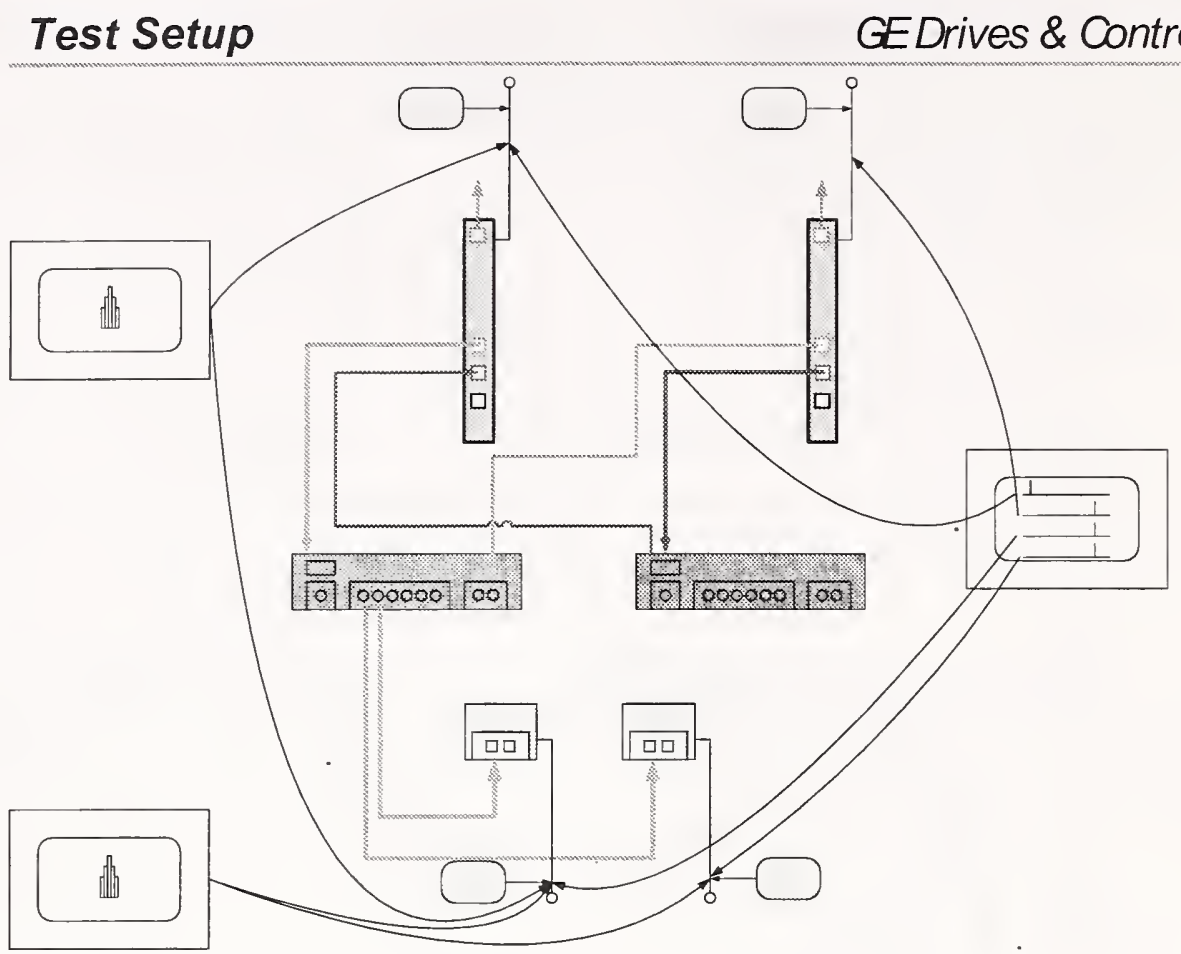

9 Master-slave delay vs time

GEDrives \& Controls, Inc.

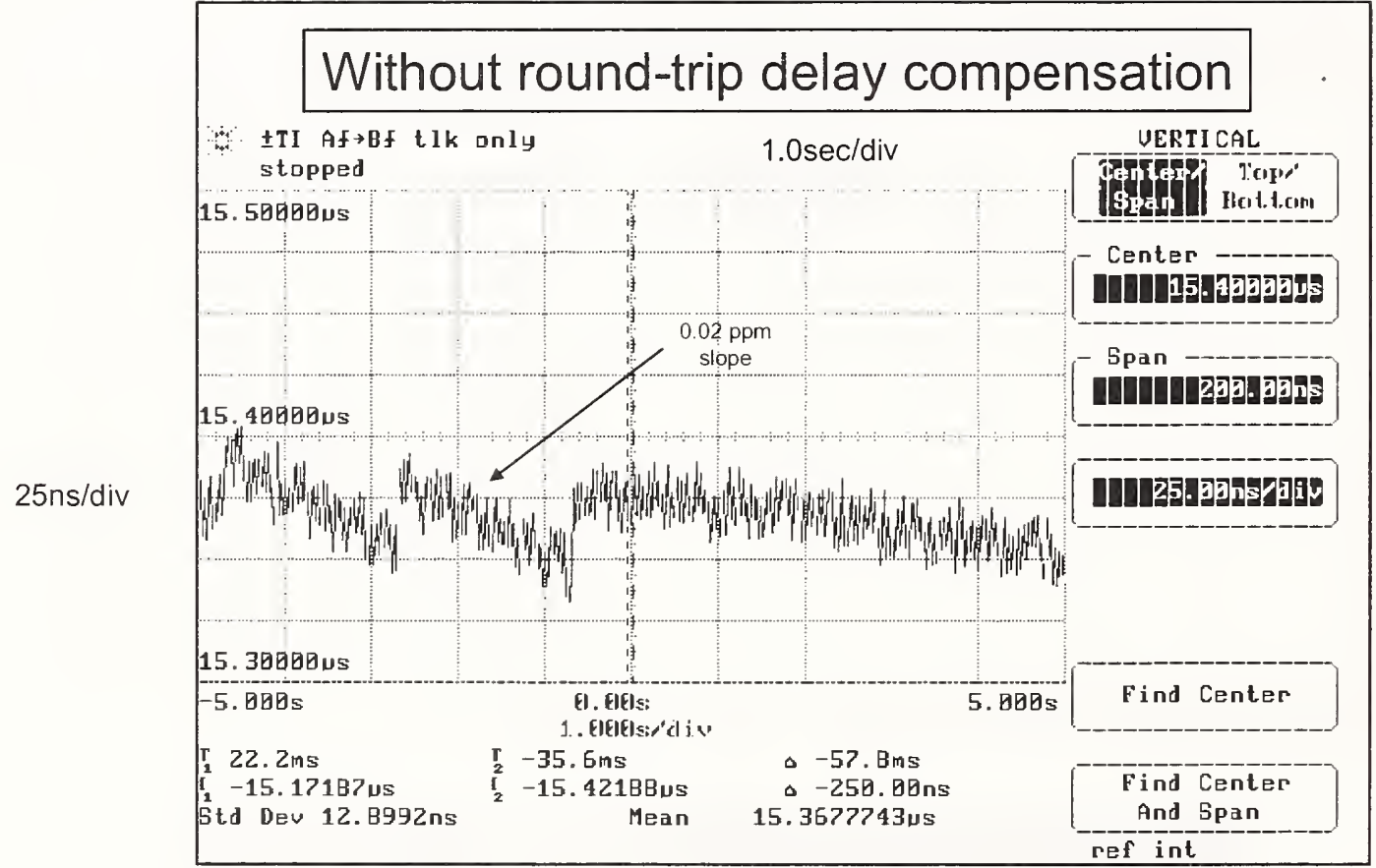




\section{O Timing with 100BaseTx repeater hub GEDrives \& Controls, Inc.}

Without round-trip delay compensation

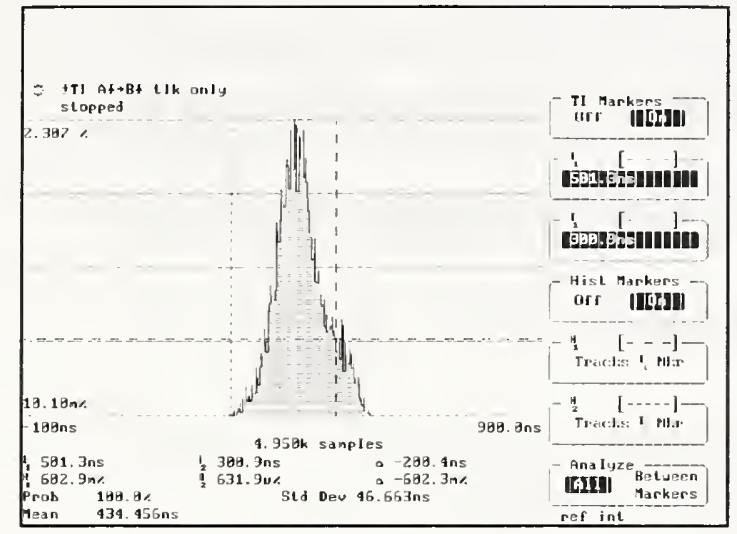

Controller-to-pack timing

$x=434 \mathrm{~ns} \quad \sigma=47 \mathrm{~ns}$

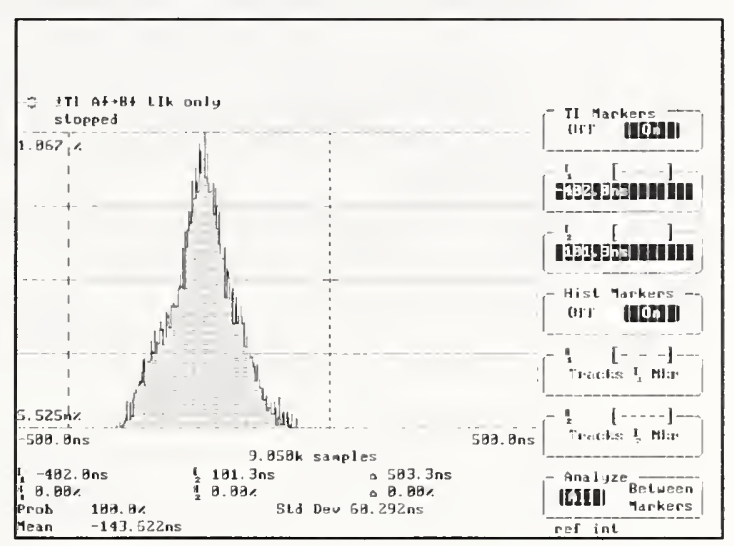

Pack-to-pack timing $\bar{x}=-144 n s \quad \sigma=60$ ns

9 Timing with 100BaseTx switch GEDrives \& Controls, Inc.

\section{Without round-trip delay compensation}
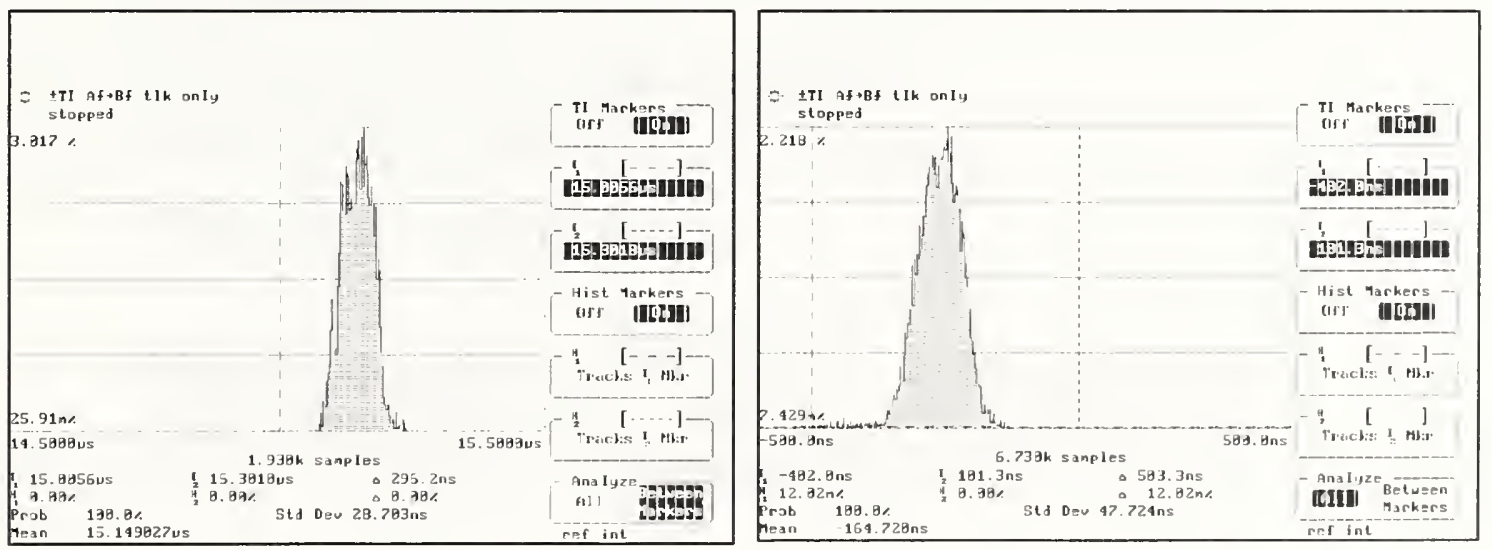

Controller-to-pack timing $x=15.15 \mu \mathrm{s} \quad \sigma=29 \mathrm{~ns}$

$$
\begin{aligned}
& \text { Pack-to-pack timing } \\
& \bar{x}=-165 \text { ns } \sigma=48 \text { ns }
\end{aligned}
$$




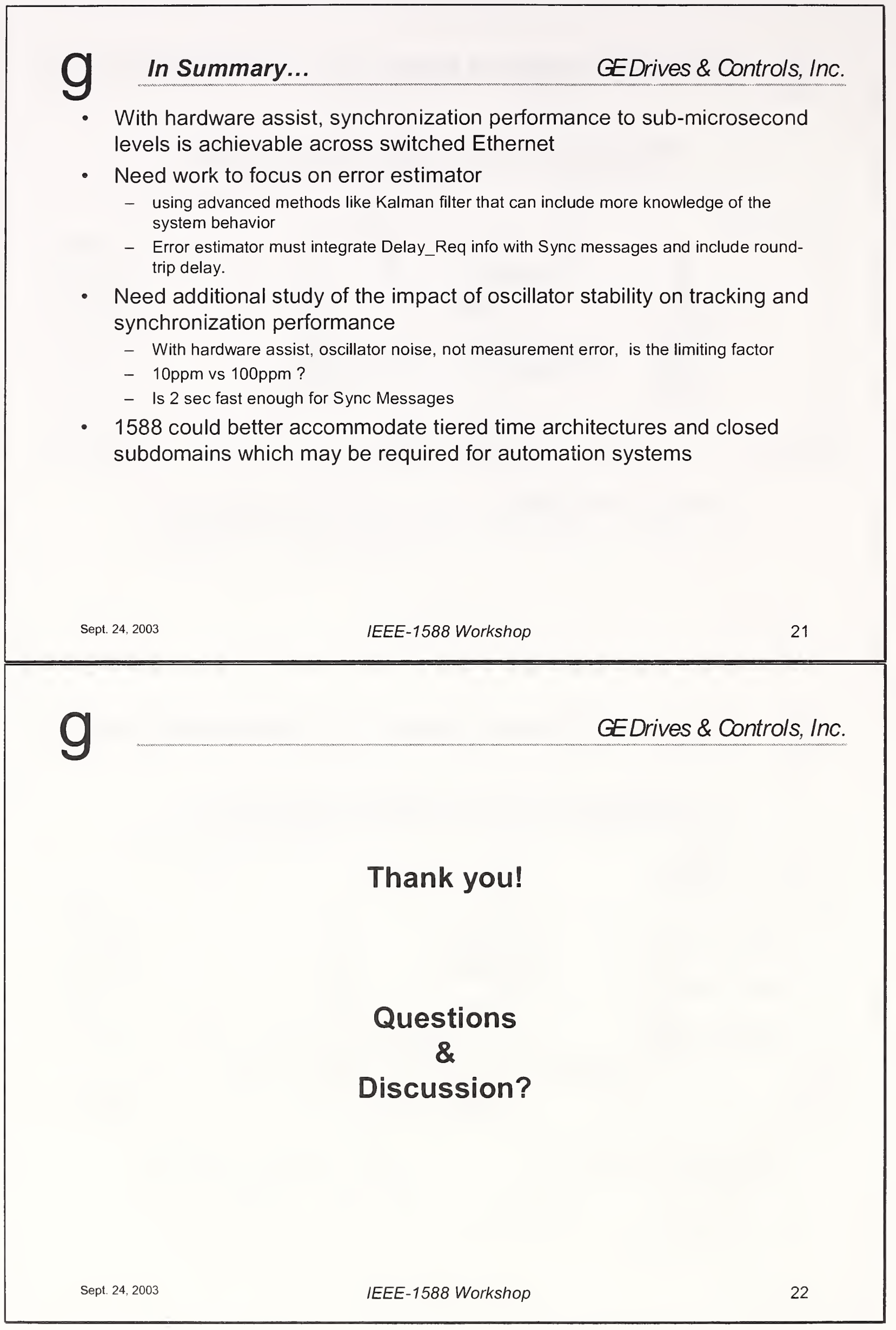




\title{
Application of IEEE 1588 to a Distributed Motion Control System
}

\author{
Ken Harris \\ Sivaram Balasubramanian \\ Anatoly Moldovansky \\ September 24, 2003
}

\section{Motion Application with 1588}

- Simple Distributed Motion Application

- 3 Controllers, 3 Motors

- 100 Mbps switched Ethernet

- 1588 Precision Time Protocol over Ethernet

- Replaces proprietary solution
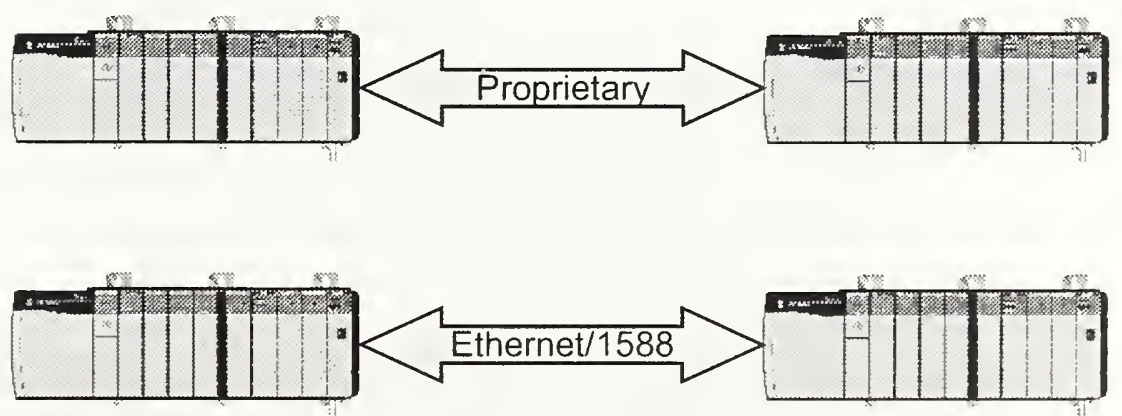


\section{Block Diagram}

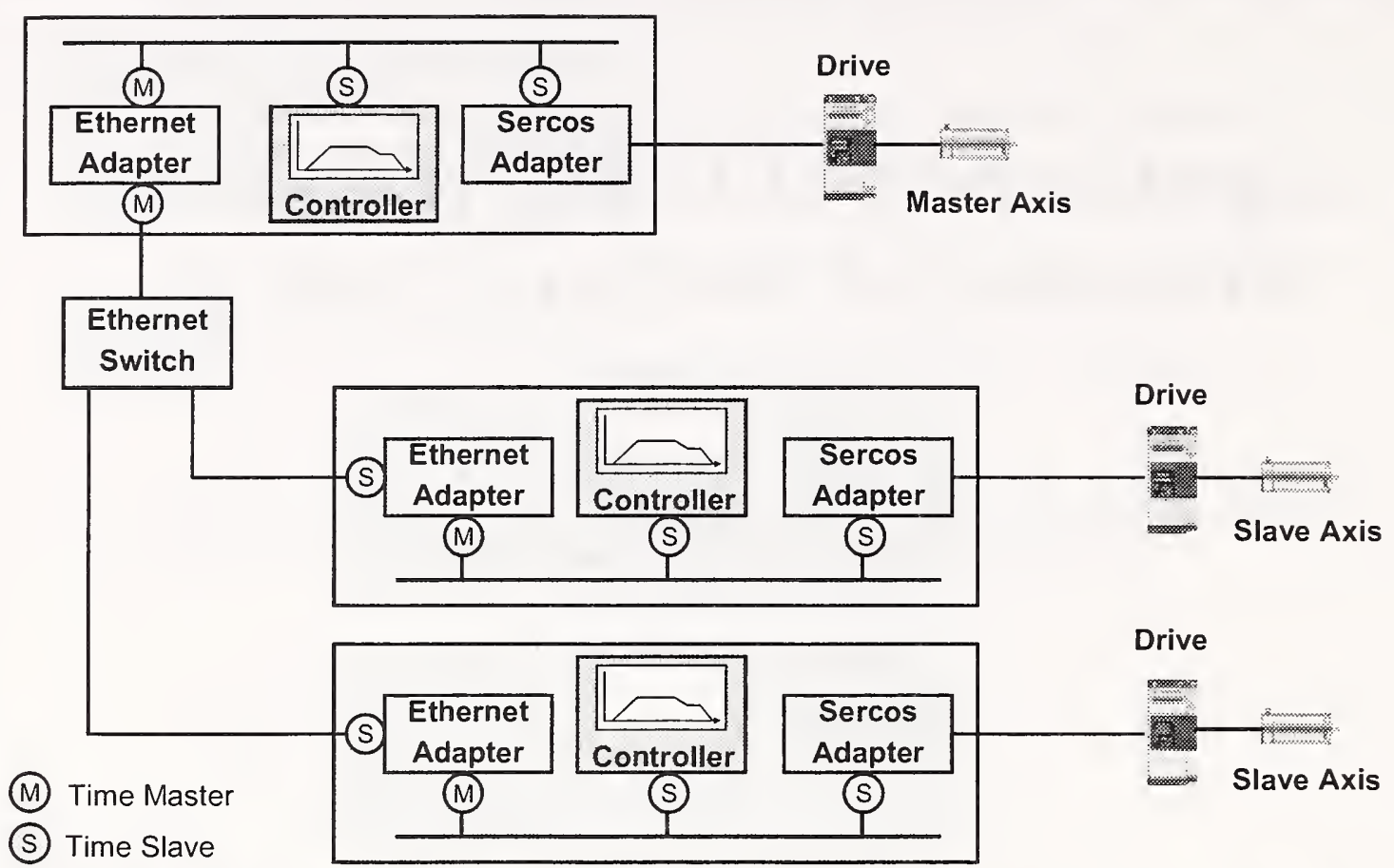

\section{Block Dlagram}

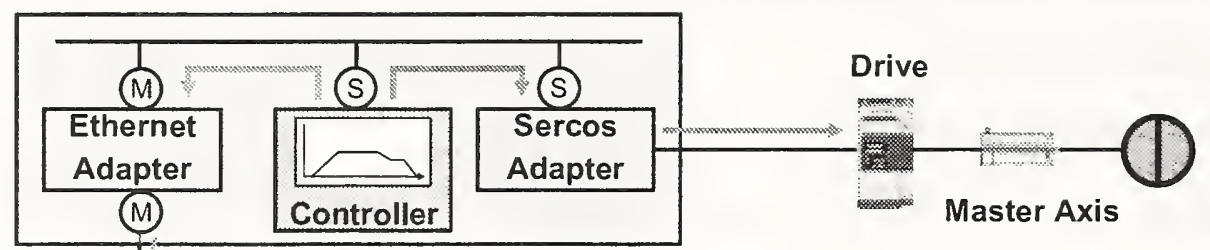

(M) Time Master

Ethernet
Switch

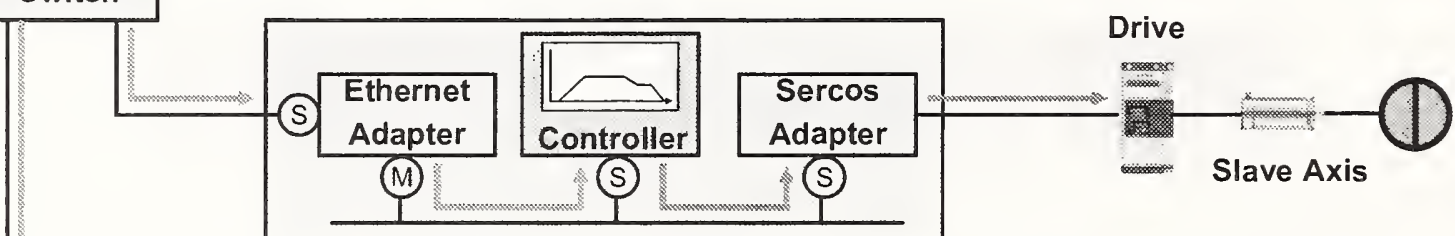

(S) Time Slave 


\section{Ethernet Adapter}

- Ethernet adapter implements 1588

- FPGA for HW assist

- PowerPC for protocol

- Backplane clock synchronized to 1588 clock

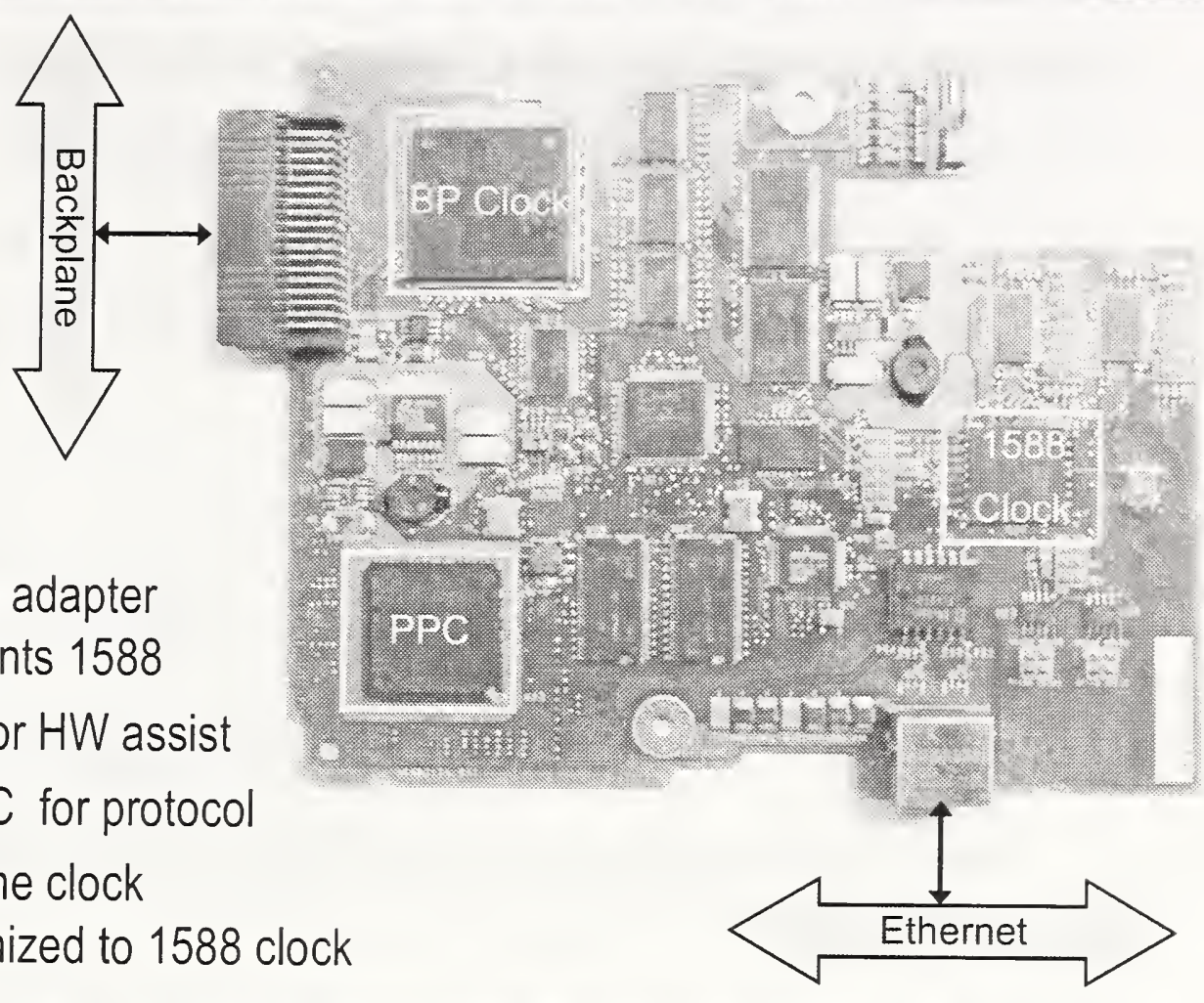

\section{Protocol Implementaton}

- 1588 implementation on Ethernet Adapter

- Hardware Assist circuit in FPGA

- Firmware Implementation of 1588 protocol

- $\mathrm{C} / \mathrm{C}++$ on $50 \mathrm{MHz}$ PowerPC

- Sync, Follow-up, Delay Request, Delay Response Messages

- Management Messages

- No "Best Master" algorithm, uses "preferred" master

- Detects loss of master

- Burst Messages supported (burst of 8)

- Boundary clock implementation to backplane clock (foreign clock)

- Results: 200 nanoseconds jitter on Ethernet 


\section{Wotion Operation}

- Motion operation requires nodes to be "synchronized"

- Transactions based on synchronized periodic update cycle

- Coniroller to Drive transactions
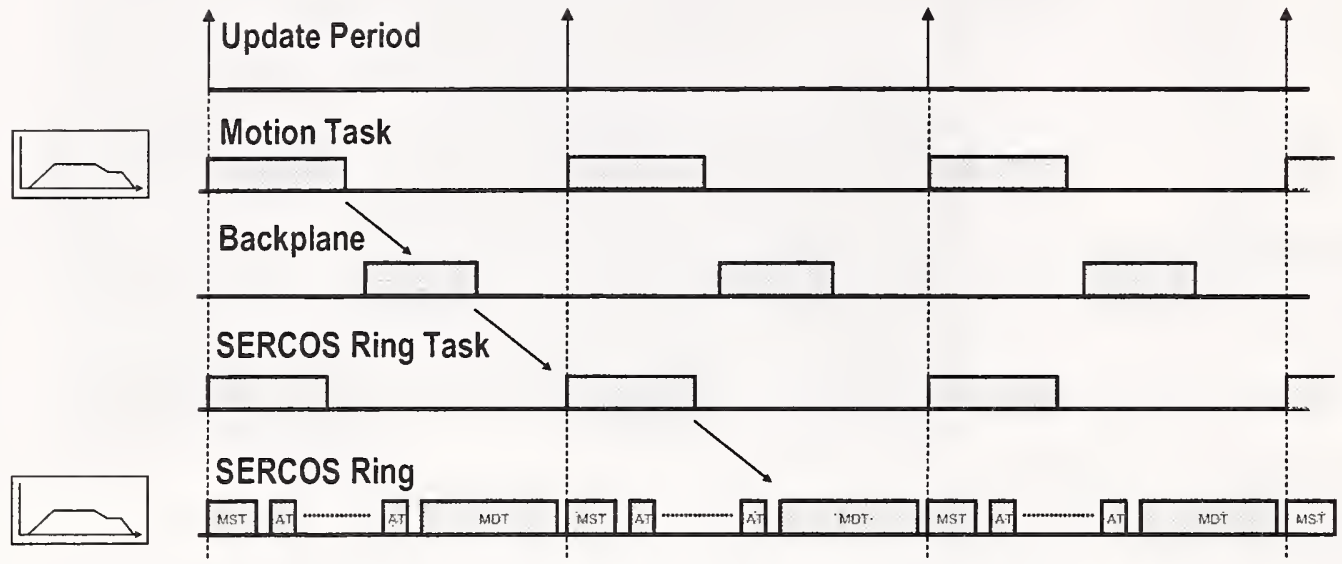

Controlen bo Orve Transactions

\section{Motion Operation}

- Motion operation requires nodes to be "synchronized"

- Transactions based on synchronized periodic update cycle

- Controller to Controller transactions

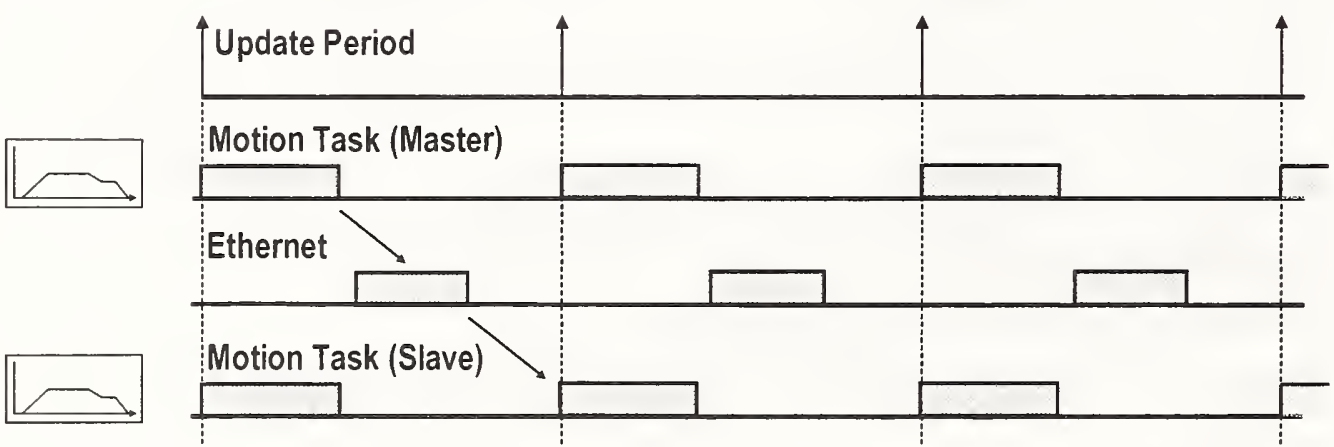




\section{Task Synchronizing}

- Tasks synchronized to 1588 clock with hardware support

- Target Time register loaded with "Update" start time

- Interrupt occurs when 1588 clock equals start time

- New target time loaded to repeat cycle

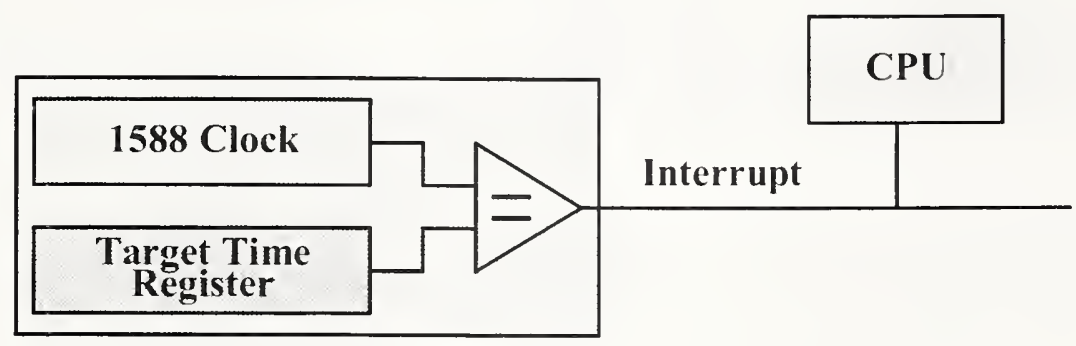

Task Synchronizing Circuit

\section{Synchronized Outputs}

- Output is asserted/deasserted based on motion position

- "Planner" interpolates on/off position and translates to on/off time

- Controller sends "schedule" to output module

- Output module asserts/deasserts output at "scheduled" time

- Output module clock is synchronized to controller clock

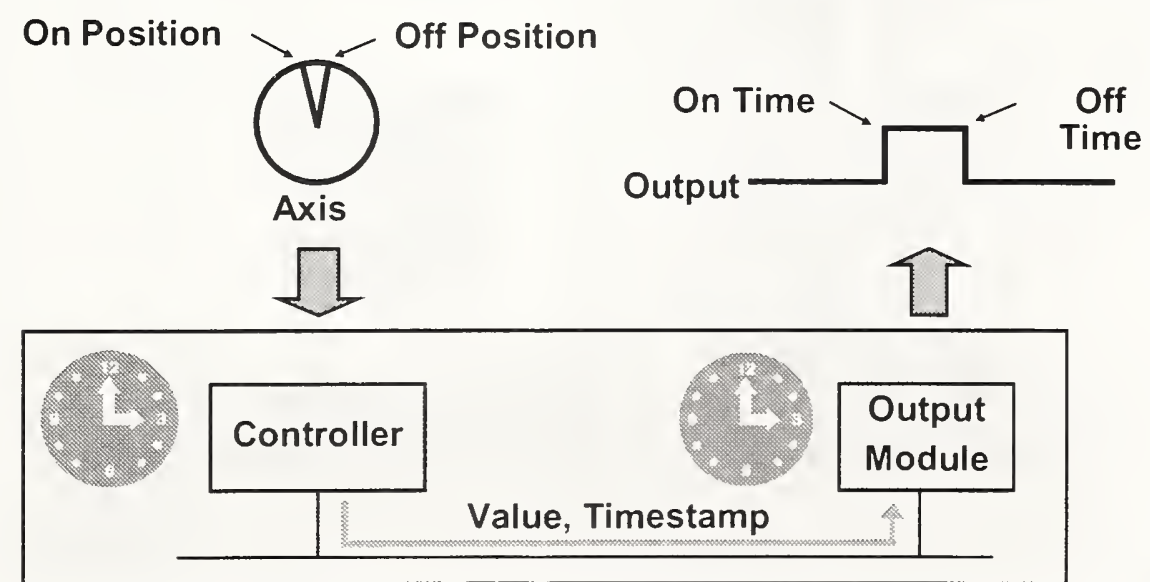




\section{GPS as Grandilaster}

- Ethernet adapter modified to interface to GPS receiver

- Receives Pulse-Per-Second and UTC from receiver

- Local 1588 clock synchronized to GPS clock

- Results: 500 nsec accumulated jitter

(M) Time Master

(S) Time Slave

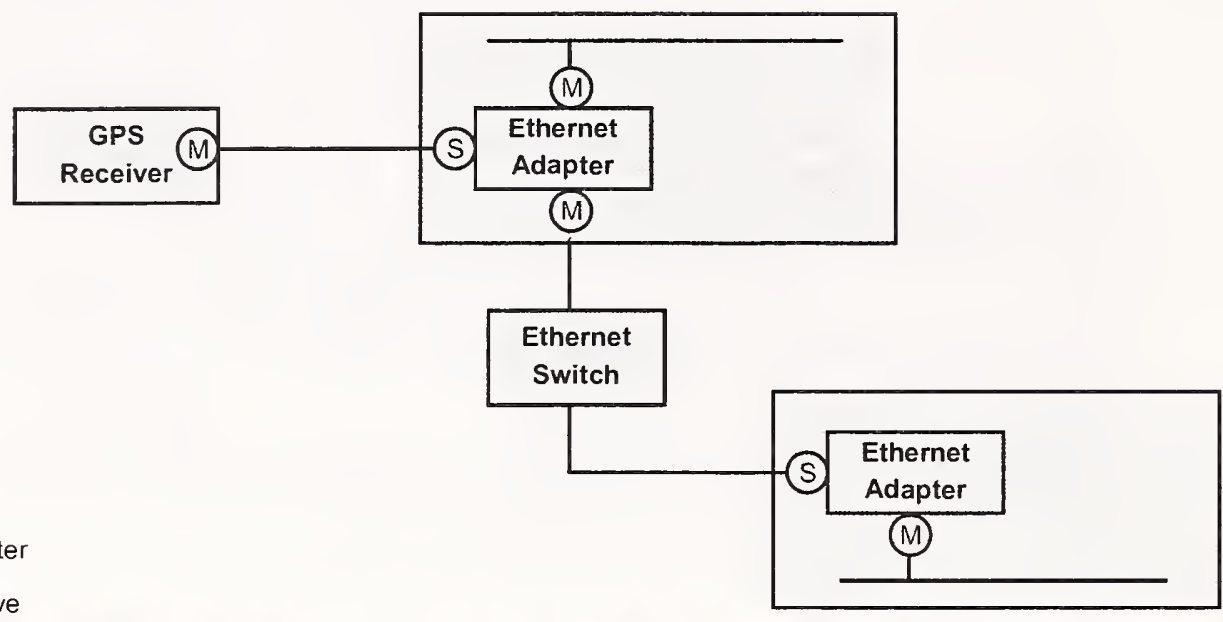

\section{Demo Pedestal}

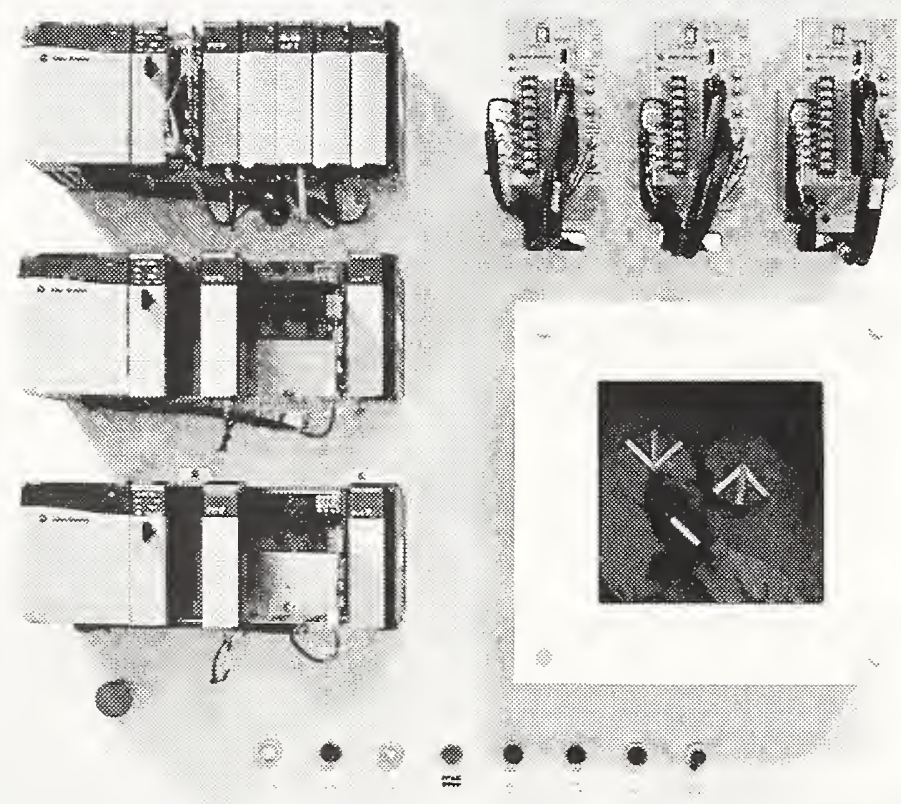




\section{IEEE1588 proposal for Metro Ethernet Enterprise Solutions}

IEEE1588 Sept 242003 workshop presentation

\section{Glenn Algie}

Nortel Networks, Wireless Technology Labs

Sept 242003

NORTEL

Why IEEE1588 Enhancements ?
Problem Statement:
- Transition is now occurring from Circuit to Packet in the Metro
- Ethernet edges are replacing the Traditional E1/T1 circuit demarcation (803.3ah)
Timing sensitive services that used the Circuit/Sonet/SDH timing
references can't transition to Ethernet edge without a packet based
Precision timing reference. New timing sensitive packet based
services are also emerging.
Solution Proposed:
NORTEL NETWORKS proposes that IEEE1588 be adapted for this
need. Positioned as a Precision timing service over Metro Ethernet
demarcations into Enterprise VPN (Virtual Private Network).
Slight enhancements to the IEEE1588 Standard are proposed here for
these Metro applications.
1588 timing payloads are extensible to any frame/cell transport.
Does not replace NTP. Interworking is expected.
IEEE1588 enables timing sensitive end services on
Enterprise VPNs to utilize Metro Ethernet Solutions
NORTEL
NETWORKS




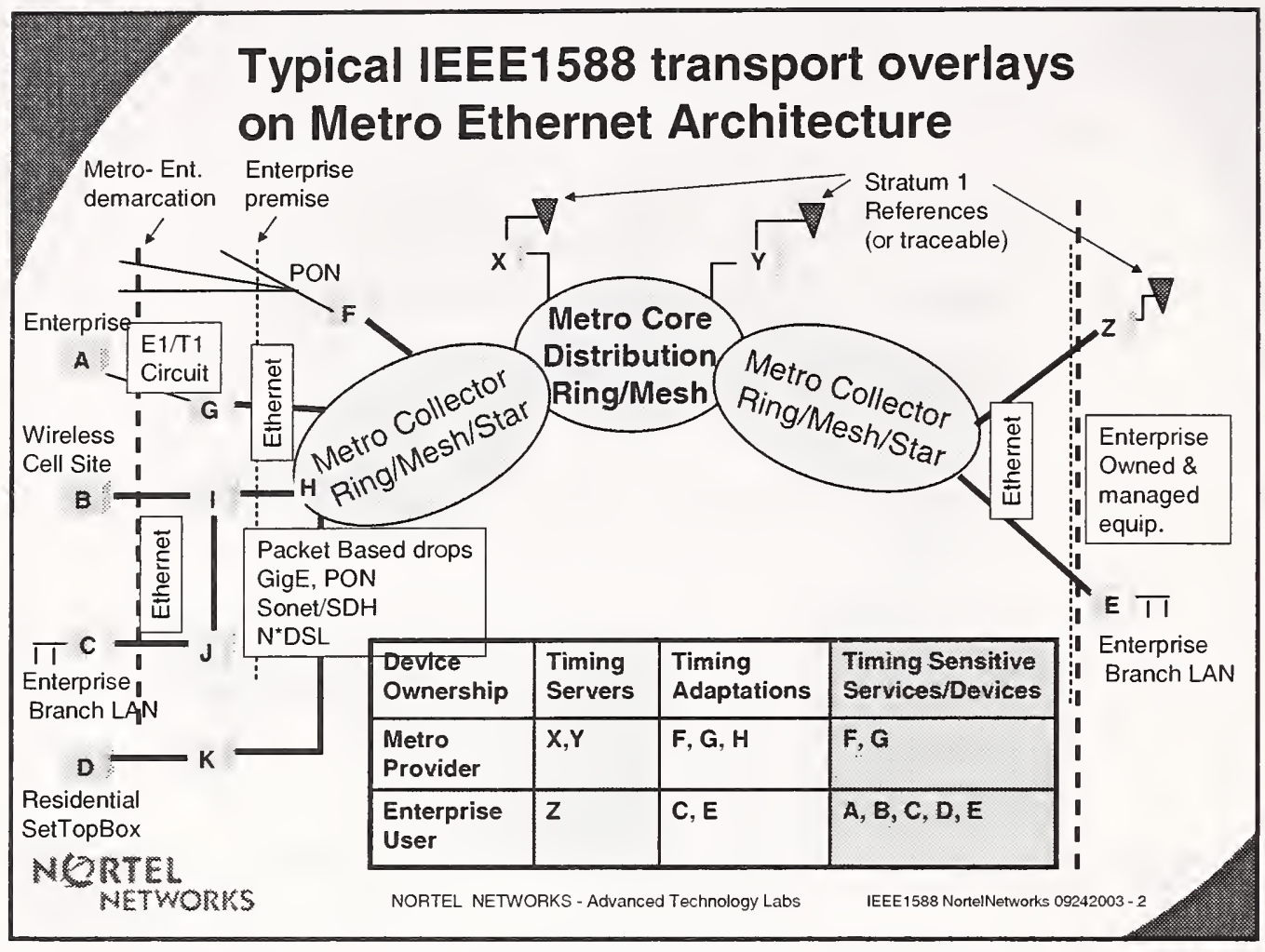

\section{Metro Ethernet 1588 Timing Service Dimensions}

Precision Timing Service Components:

- $\quad$ Timing Sourcing edge point(s)

- Timing Recovery/Adaptation edge points

- Timing Sensitive Service edge points

Management/ownership options:

- Enterprise managed endpoint

- Metro Provider managed endpoint

- Hosted Service provider endpoint

Variety of Metro Ethernet Network Technologies:

- core distribution vs. collector edge, Ring vs Mesh deployments

- $\quad$ L1/L2 transport technology variety has performance impact on IEEE1588 PTP flows

- Precision Timing Service Components span management boundaries

- A multivendor timing solution requires a Standard

- Variety of Metro Ethernet technologies affect end-end performance NORTEL 


\section{Metro Ethernet Network Technologies}

Typical Metro Network Elements:

- DWDM Metro optical cores in most cities in North America

- Variety of Mesh or Ring optical configurations in Metro Primary distribution core and Metro Secondary collectors

- Spans the dense City Metro fanning out to urban and suburban areas

- $\quad$ Further subtending pt-pt and pt-mpt optical and copper hybrid circuits/packet based feeders

Metro Ethernet Ring/Mesh Transport technologies \& influences

- Ethernet over Sonet/SDH (X.86/GFP) - Sonet/SDH switched at each hop

- $\quad$ Ethernet/MPLS Mesh - L2 switched/queued at each hop

- RPRMAC (802.17) - L2 switched at the edge then express forwarding around the ring .

Metro Ethernet last hop technologies

- $\quad$ Native Ethernet (802.3ah), Ethemet over DSL

- $\quad E 1 / T 1 / T 3$ Circuit interfaces still at the Small/Medium Enterprise premises

- End User/Enterprise Ethernet MAC layer is transparently bridged over the Metro Ethernet

- Metro Ethernet deployment options are Metro Provider specific

- A Variety of Packet and Optical technologies are used

- Impairments to the bridged Ethernet MAC can be quantified

NORTEL

METWORKS

NORTEL NETWORKS - Advanced Technology Labs

IEEE1588 Nortel Networks 09242003 - 4

\section{Metro Ethernet Technologies impacts on IEEE1588 PTP flows}

- PTP Flows impacted with:

- Fixed Packet Latency, plan for 0.5 - 50 Millisecond

- Variable Packet delays, "packet jitter", plan for 0.5 - $3.5 \mathrm{Msec}$

- Novel Techniques exist that can tolerate and filter out these impacts in the timing service components.

- Packet QOS treatments in the Metro edges and core switching points exist to minimize packet forwarding affects.

- In progress Nortel Technology Labs field test findings show the affects can be filtered and recovered to parts per billion level of stability on typical Metro Ethernet Solutions!

- Nortel Technology Lab running it now over a typical public Ottawa (Ontario Canada) Metro Ethernet service.

- A multi tiered DWDM and RPRMAC and L2 switched cumulative set of technologies applied. 20-30 Metro/Enterprise devices.

- Modern technologies and techniques can tolerate Metro Network affects on the precision timing packets

NORTEL

NETWORKS 


\section{IEEE1588 impacts to enable a Metro Ethernet Enterprise VPN use case.}

Key revision items needed for a Metro Ethernet use of 1588:

- Capture Metro Ethernet "Boundary clock" deployment rules

- Add packet latency and packet delay variation boundary conditions.

- Allow subsecond rate granularity of the timestamps based messages.

- Propose a new bit/field parameter in spare octet of Sync, Delay_req message.

- Bit " 0 " value means existing seconds based granularity for the existing "rate" field.

- Bit "1" value means 10 or 100 millisecond based interval rate for the existing "rate" field.

Additional Standards items:

- Seek necessary reserved identifiers such as Ethertype and/or Multicast from IEEE Registration Authority for IEEE1588 payloads.

- Is a unique Ethernet multicast address enough?

- Some scenarios deployable where no UDP/IP stack exists!

- ITU-T SG15 Q13 address synchronization service needs over asynchronous (packet) transport. Packet based timing transport rules could be worked here.

- Informative presentations into MEF, ITU-T, 802..., and Telecom Industry conferences as appropriate.

\section{NORTEL}

\section{Next steps}

- Seek IEEE1588 and Telecom Industry user/vendor sponsorships for this proposal.

- Propose to IEEE1588 Chair for a PAR revision project.

- Review by Registration Authority, include new Ethertype and/or multicast address needs in the request

- Liaisons and informative presentations as needed with IEEE802.3, 802.1, 802.17, MEF and ITU-T SG15. Also other industry conferences as appropriate to spread the word.

\section{Tybom Indusing Target objective:}

- Enable timing sensitive edge services to utilize the cost effective value of Metro Ethernet solutions.

- Make the packet timing service a "no brainer" for Metro Enterprise solutions. Make it better or equal to the network derived timing performance of legacy E1/T1/Sonet/SDH that Metro - Enterprise demarcations had. 


\section{Backup slide - Proposed revision to "synclnterval" octets}

- Backward compatible enhancement to Sync and Delay_Req Messages

- Add a "intervalGranularity" octet into spare octet

- intervalGranularity definition:

0 : second (default)

1: 10 millisecond

2: 1 millisecond

\begin{tabular}{|l|l|l|l|l|l|l|l|l|}
\hline \multirow{2}{*}{ SOF } & $\mathrm{N}$ & $\begin{array}{l}\text { Octet } \\
\mathrm{N}\end{array}$ & $\begin{array}{l}\text { Octet } \\
\mathrm{N}+1\end{array}$ & $\begin{array}{l}\text { Octet } \\
\mathrm{N}+2\end{array}$ & $\begin{array}{l}\text { Octet } \\
\mathrm{N}+3\end{array}$ & $\begin{array}{l}\text { Type } \\
\text { (Informative) }\end{array}$ & $\begin{array}{l}\text { Field Name } \\
\text { Clause 8.2 and 8.3 }\end{array}$ \\
\cline { 2 - 8 } proposed & 122 & 80 & 00 & 00 & 00 & $\mathrm{H} 0 \mathrm{H} 1$ & Integer8 & synclnterval \\
\cline { 2 - 7 } & 122 & 80 & 00 & $\mathrm{~K} 0 \mathrm{~K} 1$ & 00 & $\mathrm{H} 0 \mathrm{H} 1$ & $\begin{array}{l}\text { Octet| Integer8| } \\
\text { Octet| Integer8 }\end{array}$ & $\begin{array}{l}\text { intervalGranularity| } \\
\text { synclnterval }\end{array}$ \\
\hline
\end{tabular}

NORTEL

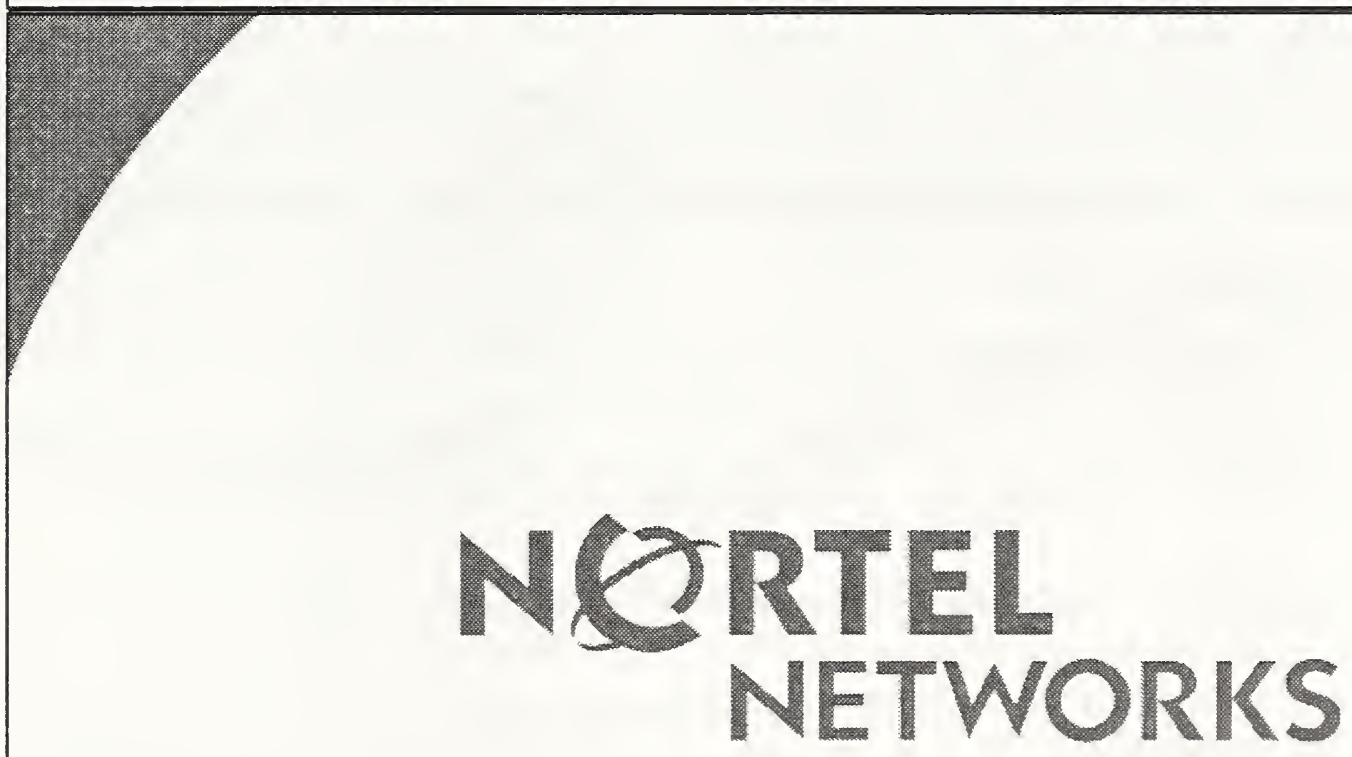




\section{Appendix A}

The followings are approximate transcripts of the questions and answers following each of the presented papers at the IEEE 1588 workshop held on Sept. 24, 2003.

\section{Paper 1: Boundary Clock Implementation: Holmeide.}

Q: How does flow control influence the access to the Ethernet and synchronization? Holmeide: This relates to the specifics of an Ethernet switch chip set and whether it can be used to disable communication. I don't want to end up with the drop link being disabled just when I wand to send a crucial time sync packet. So flow control is useful not for halting general traffic but rather to control synchronization traffic... It can be a tool to help be sure the switch is silent in preparation for sending a sync packet. Q: Do we need the hardware assist and the accuracies in industrial controllers and silicon?

Holmeide: Most applications are targeting milliseconds and will be for a long time therefore most will be enabled in software. I have seen initiatives from silicon manufactures to implement the hardware and that will be great. The number of gates needed is small.

\section{Paper 2: Extending IEEE 1588 to fault tolerant synchronization with a worst-case precision in the 100 ns range: Kero, Holler and Sauter.}

Q: What is the difference between using time information from all clocks vs. a master slave principle as in 1588 ,

Kero: If you have a guaranteed master clock with say an atomic clock and a 1588 net then you are in the same range and you are fine. However if you have a few clocks of different quality and maybe one fails then the transient will be worse. An ensemble may work better in this case.

Q: Processing overhead?

Kero: It is not an issue since the sync is only every 10 seconds.

Q: The issue is not the delay through the PHY it is the asymmetry.

Kero: We know all the relevant times in transit through the switch so we know all the needed questions. (There were further questions deferred due to time)

\section{Paper 3: Consequences of Redundant Structures PTP: Winkel.}

Q: Question about the kinds of redundancy being considered.

Winkel: The rapid spanning tree is a fine solution for high-end systems but in industrial automation we need 10-millisecond recovery.

Q: Is the SC65 proposal going to be a competing standard?

Winkel: We would like to open an IEEE PAR to address this issue. In SC65 the view is wider, beyond time sync. SC 65 is to define what real-time means and then to bring the issues to the 1588 committee.

\section{Paper 4: A solution for fault-tolerant IEEE 1588: Allan, Lee}

\section{Q: No questions}


Paper 5: Impact of Switch Cascading on Time Accuracy: Mueller, Weber

Q: You are proposing eliminating UDP and IP and running directly over Ethernet Weber: Yes

\section{Paper 6: IEEE 1588 and Network Devices: Mohl.}

Q: Your SNMP proposal is it similar to the 1588 equivalent and do you implement traps? Mohl: It covers the same information and we do not implement traps at present.

Paper 7: A frequency compensated clock for Precision synchronization using IEEE 1588 protocol and its application to Ethernet: Balasubramanian, Harris, Moldovansky.

Q: What about start up where there may be large differences?

Moldovansky: There needs to be a separate algorithm for big start up situations. We see at most 6-7 seconds pull in for the transients we see. Rockwell has a proprietary system that does have a fast pull in for start up. We may apply it to this environment as well.

\section{Paper 8: IEEE-1588 Node Synchronization Improvement by High Stability Oscillators: Eidson, Hamilton.}

Q: Is there provision for a burst mode in the protocol to allow compensation for some of the effects mentioned today?

Eidson: There is a burst mode in the standard allowing either slaves or master to increase the rate of sync message. It was put in to allow more rapid recovery in the event of the need to recompute parameters.

Q: In the direct case you reported 15 ns standard deviation with good oscillators. What could be done to improve this?

Eidson: The oscillators we used had x 1000 headroom. To do better you will need a finer resolution on the clock. I am not optimistic about reaching one or two nanoseconds.

Paper 9: Time Correlation using network based data acquisition onboard a Military Test Vehicle: Dai, DeSelms, Grozalis.

Q: (Question could not be heard on the tape)

Dai: We have built the first prototype to bridge to existing systems. We have not completed the time synchronization portion.

Q: We had same connector problem.

Dai: The problem is standardization on a single connector.

Paper 10: Implementation of IEEE Std.-1588 in a Networked I/O Node: Shepard.

Q: Did you tweak the standard?

Shepard: We did not tweak it but we may have used elements in odd ways.

Paper 11: Application of IEEE 1588 to Distributed Motion Control: Harris, Balasubramanian, Moldovansky. 
Q: Being a part of Rockwell are you part of the CIPSync definition

Harris: Yes

Q: How did you use burst?

Harris: We used it to speed up the clock synchronization during pull in.

Q: How much did this help?

Harris: We improved from 20 -second pull in to about 4 seconds.

\section{Paper 12: Proposal for IEEE 1588 use over Metro Ethernet Layer 2: Algie.}

Q: Operating with voice packet networks that use RTP?

Algie: RTP does include timestamps but only to milliseconds alignments. We use NTP derived clock now. Think 1588 will interwork with these. My concern is with video and similar.

Q: (Question could not be heard on the tape)

Algie: For voice over IP could use derived clock from 1588 but could use NTP. For more precise things like cell sites we think we can use 1588.

Q: Are your pictures US centric or worldwide?

Algie: It is similar everywhere. 


\section{IEEE 1588 Prototype Demonstration}

Hirschmann Electronics $\mathrm{GmbH} \& \mathrm{CO} . \mathrm{KG}$ Automation and Network Solutions

T. Gramann

IEEE 1588 Network

(A) Boundary Clock as Grandmaster

$\left(\begin{array}{l}\text { Boundary } \\ \text { clock }\end{array}\right.$

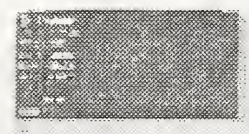

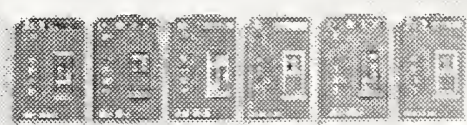

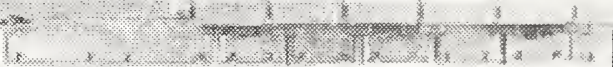
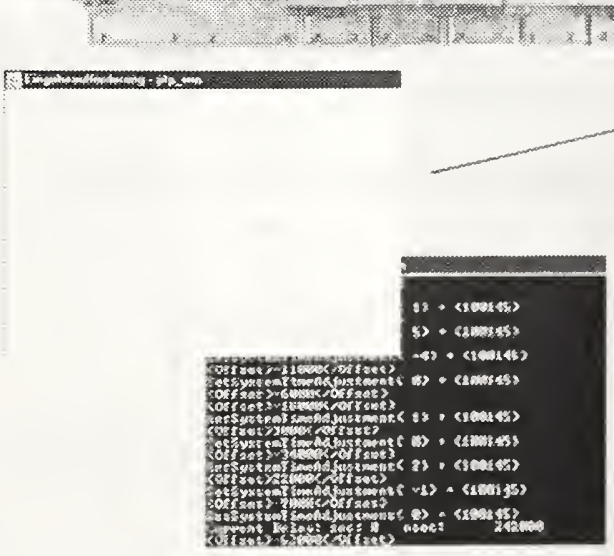

Ordinary Clock 


\title{
Demonstration for the Application of IEEE-1588 to Distributed Motion Control
}

\author{
Steven A. Zuponcic
}

Rockwell Automation

September 24, 2003

$\frac{5}{20}$

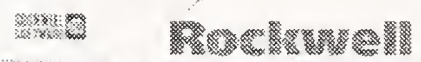
Automatron

\section{EEE-1588 Demo for Distributed Motion Control}

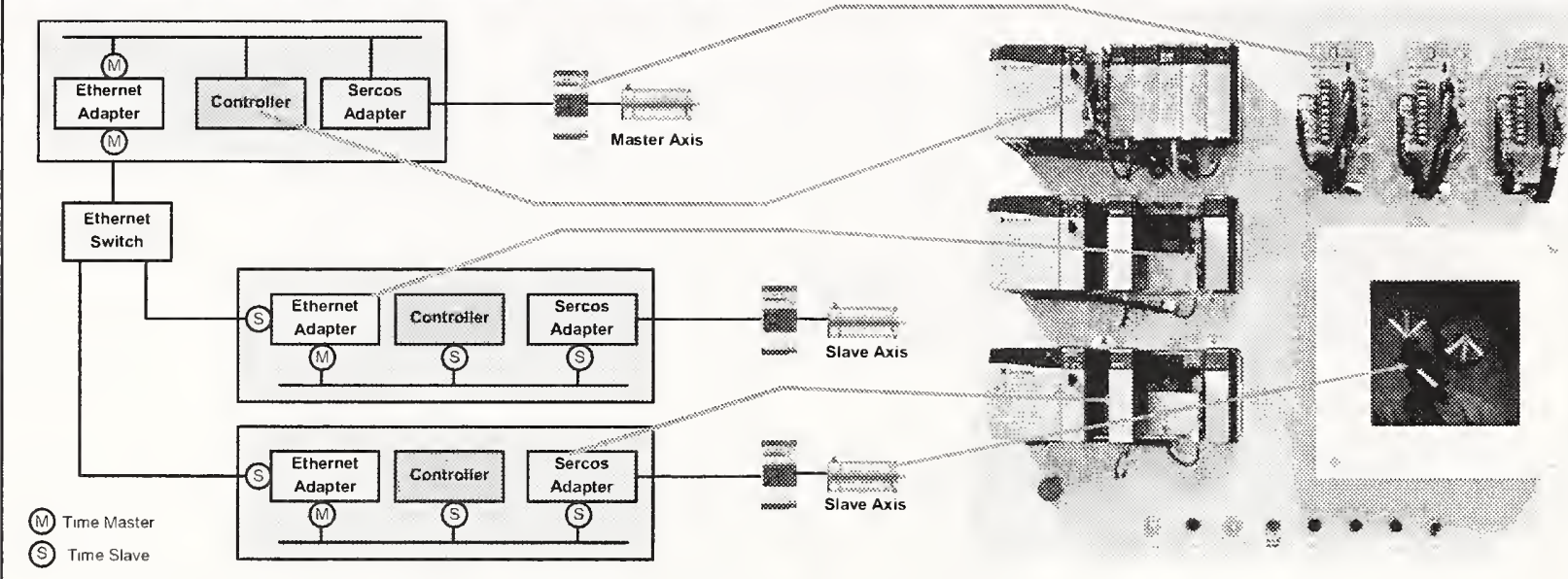

- Distributed Chassis Clocks are Synchronized to within 100ns of each other

- Motion Reference Data is Produced in the Master Chassis and Consumed in the Slave Chassis

- Task Execution in Master Chassis is Synchronized with Task Execution in Slave Chassis to Produce Smooth, Precise, Coordination 


\section{Selup to show etrects of oscillator qually on synchronization}

Agilent Technologies prototype clock

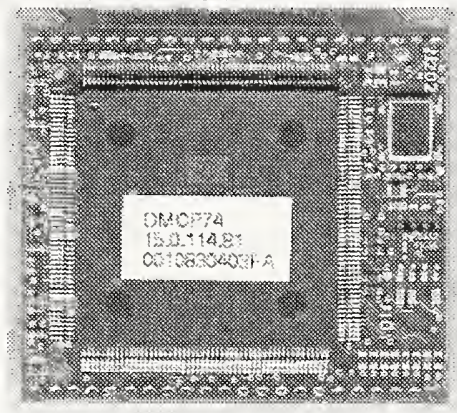

Clock resolution: 25 ns

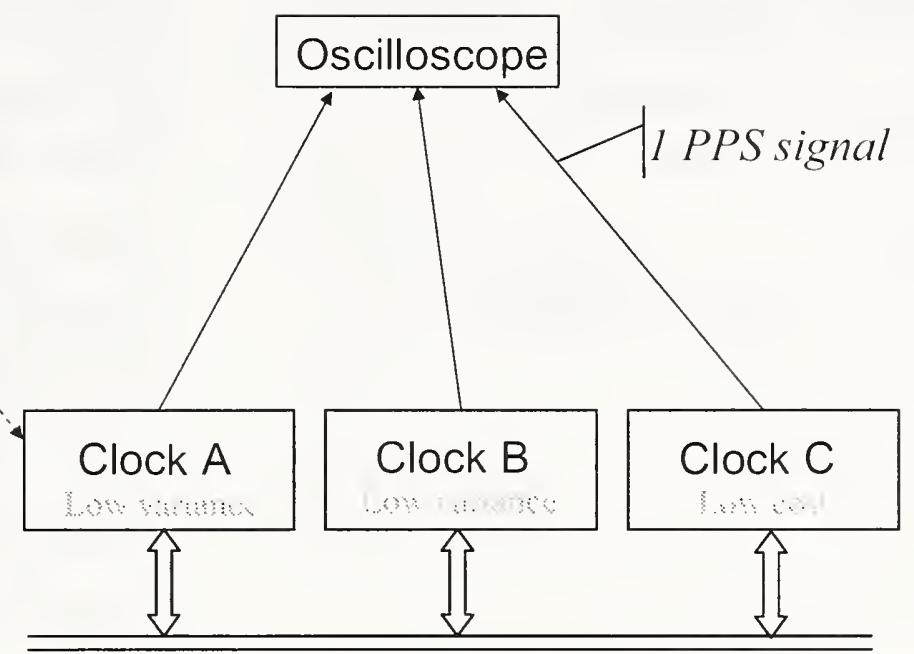




\section{Final Participants List}

Glenn Algie

Nortel Networks

3500 Carling Ave.

Ottawa, Ontario, K2H 8E9

CANADA

(613) 763-2211

algie@nortelnetworks.com

Geoffrey Allan

Dependable Real Time Systems Ltd.

The Innovation Centre

Sheffield S1 4Dp,

UNITED KINGDOM

(44) 1142232301

44/1142232301

jeff.allan@drts.co.uk

Fred Balady

Intel Corporation

1515 Route Ten

Parsippany, NJ 07054

USA

(973) 967-6617

fred.balady@intel.com

Christopher Chang

Netsilicon, Inc.

400 Waverley Oaks Road

Waltham, MA 02452

USA

(781) $647-1234$

781/893-1338

cchang@netsilicon.com

Muhammad Saeed Chaudhry

Nortel Networks

20410 Century Blvd.

Germatown, MD 20874

USA

(301) 528-3233

301/528-3298

mchaud@nortelnetworks.com
Jiwang Dai

L-3 Communications

1515 Grundy's Lane

Bristol, PA 19007

USA

(267) 545-7037

jiwang.dai@1-3com.com

Patrick Diamond

Semtech Corp.

200 Flynn Road

Camarillo, CA 93021

USA

(805) 480-2188

$805 / 498-5621$

pdiamond@semtech.com

John Eidson

Agilent Technologies

3500 Deer Creek Rd.

Palo Alto, CA 94304

USA

(650) 485-4263

650/485-3894

john_edison@agilent.com

Mark Elliott

Symmetricom

3750 Westwind Blvd.

Santa Rosa, CA 95403

USA

(707) 636-1931

melliot@symmetricom.com

Doug Fowley

GE Industrial Systems

1501 Roanoke Blvd.

Room 281

Salem, VA 24153

USA

(540) 387-8598

540/387-8651

douglas.fowley@indsys.ge.com 
Michael Gerstenberger

KUKA Development Labs

2619 Product Drive

Suite 104

Rochester Hills, MI 48309

USA

(248) 844-0561

248/853-0707

michaelgerstenberger@kukadevlabs.com

Grant Gilchrist

GE Energy Services

2728 Hopewell Place, NE

Calgary, Alberta, T1Y 1 G6

CANADA

(403) 214-4519

403/287-7946

grant.gilchrist@ps.ge.com

James Gilsinn

NIST

100 Bureau Drive

Mail Stop 8230

Gaithersburg, MD 20899-8230

USA

(301) 975-3865

301/990-9688

gilsinnj@cme.nist.gov

Barbara Goldstein

NIST

100 Bureau Drive

Mail Stop 8103

Gaithersburg, MD 20899-8103

USA

(301) 975-2304

301/975-4091

barbara.goldstein@nist.gov

Chris Guo

$3 \mathrm{eTI}$

700 King Farm Blvd.

Rockville, MD 22182

USA

(301) 944-1294

eguo@3eti.com
Bruce Hamilton

Agilent Technologies

Box 10350

MS 24M-A

Palo Alto, CA 94303

USA

(650) 485-2818

650/485-4917

bruce_hamilton@agilent.com

Ken Harris

Rockwell Automation

1 Allen-Bradley Dr.

Mayfield Hts., $\mathrm{OH} \quad 44124$

USA

(440) 646-3621

440/646-3258

krharris@ra.rockwell.com

Oyvind Holmeide

OnTime Networks AS

Gladsvei 20

Oslo 0489,

NORWAY

(47) 22090304

47/22090310

oeyvind@ontimenet.com

W. Harrell Huckeba

Symmetricom, Inc.

3750 Westwind Blvd.

Santa Rosa, CA 95403

USA

(707) 636-1937

hhuckeba@symmetricom.com

Dominic Iadonisi

Hirschmann USA

55 Oakmont

Auburn Hill, MI 48326

USA

(248) 852-0338

diadonisi@hirschmann-usa.com 
Roy Jackson

GE Industrial Systems

1501 Roanoke Blvd.

Salem, VA 24153

USA

(540) 387-8417

roy.jackson@indsys.ge.com

Robert Johnson

Telemonitor, Inc.

9055F Guilford Road

Columbia, MD 21046

USA

(410) 312-6621

410/312-6653

robertj@telemonitor.com

John Kates

JXI2, Inc.

2308 Lake Omega St.

El Paso, TX 79936

USA

(915) 856-0241

915/856-0245

jkates@elp.rr.com

Krishna Kaza

3e Technologies International

700 King Farm Blvd.

Suite 600

Rockville, MD 20850

USA

(301) 944-1306

301/670-6989

kkaza@3eti.com

Nikolaus Keroe

Oregano Systems Ltd.

Phorusgasse 8

Vienna A-1040,

AUSTRIA

(43) 6763126964

43/856539914

keroe@oregano.at
Dennis King

GE Industrial Systems

1501 Roanoke Blvd.

Salem, VA 24153

USA

(540) 387-8322

540/387-8651

dennis.king@indsys.ge.com

Kang Lee

NIST

100 Bureau Drive

MS 8220

Gaithersburg, MD 20899-8220

USA

(301) 975-6604

301/990-3851

kang.lee@nist.gov

Ya-Shian Li

NIST

100 Bureau Drive

Mail Stop 8114

Gaithersburg, MD 20899-8114

USA

(301) 975-5319

ya-shian.li@nist.gov

John MacKay

Progeny Systems, Inc.

8809 Sudley Road

Suite 101

Manassas, VA 20110

USA

(703) 368-6107

703/331-5651

john.mackay@progeny.net

Li Mingfu

Telecommunication Labs

No 12, Lane 551

Min-Tsu Rd Sec 5, Yang-Mei

Taoyuan 326,

TAIWAN

(886) 34245181

$886 / 34245474$

mfli@cht.com.tw 
Dirk Mohl

Hirschmann Elect. GmbH \& Co. KG

Stuttgarter Str. 45-51

Neckartenzlingen, 72654

GERMANY

(49) 7127141622

49/71271400

dmohl@nt.hirschmann.de

Anatoly Moldovansky

Rockwell Automation

1 Allen-Bradley Dr.

Mayfield Hts., OH 44124

USA

(440) 646-5369

440/646-3076

amoldovansky@ra.rockwell.com

David Moyer

Semtech Corporation

958 Spring City Road

Phoenixville, PA 19460

USA

(610) 792-9395

610/948-2945

dmoyer@semtech.com

Lee $\mathrm{Ng}$

Agilent Technologies

3500 Deer Creek Road

Palo Alto, CA 94304

USA

(650) 485-5726

650/485-6522

leenng@agilent.com

Wanda Reiss

NetSilicon

411 Waverley Oaks Road

Suite 304

Waltham, MA 02452

USA

(781) 398-4508

wreiss@netsilicon.com
Silvana Rodrigues

Zarlink Seminconductor

400 March Road

Ottawa, Ontario, $\mathrm{K} 2 \mathrm{~K} 3 \mathrm{H} 4$

CANADA

(613) 270-7590

613/592-1010

silvana.rodrigues@zarlink.com

Martin Rostan

Beckhoff

Ostendstr 196

Nuremberg 90482,

GERMANY

(49) 9115405611

49-9115405629

m.rostan@beckhoff.com

Michael Rupert

Zarlink Semiconductor

400 March Road

Ottawa, Ontario, $\mathrm{K} 2 \mathrm{~K} 3 \mathrm{H} 4$

CANADA

(613) 270-7347

michael.rupert@zarlink.com

Paul Rushton

Semtech Corp.

Units 2 \& 3

Park Court, Premier Way

Romsey, Hampshire, S051 9DN

UNITED KINGDOM

(44) 1794527600

441794527601

prushton@semtech.com

Mark Shepard

GE Drives \& Controls, Inc.

1501 Roanoke Blvd.

Salem, VA 24018

USA

(540) $387-8710$

mark.shepard@indsys.ge.com 
Paul Skoog

Symmetricom

3750 Westwind Blvd.

Santa Rosa, CA 95403

USA

(707) 636-1955

707/527-6640

pskoog@symmetricom.com

Eugene Song

NIST

100 Bureau Drive

MS 8220

Gaithersburg, MD 20899-8220

USA

(301) 975-6542

301/990-3851

ysong@cme.nist.gov

James St. Pierre

NIST

100 Bureau Drive

Mail Stop 8111

Gaithersburg, MD 20899-8111

USA

(301) 975-2403

301/948-5796

jimstp@nist.gov

Bradley Van Eck

International SEMATECH

2706 Montopolis Drive

Austin, TX 78741

USA

(512) 356-3981

512/356-7848

brad.van.eck@sematech.org

Milan Vlajnic

Nortel Networks

20410 Century Blvd.

Germantown, MD 20874

USA

(301) 528-3228

milan@nortelnetworks.com
Karl Weber

Siemens A\&D SE

Wuerzburger Str 121

Fuerth 90766,

GERMANY

(49) 1712307569

49/9117502100

karl.weber@siemens.com

Adam Wertheimer

Telcordia Technologies

100 North Road

Chester, NJ 07930

USA

(973) 829-2635

973/829-2622

adam.wertheimer@telcordia.com

Joseph White

U.S. Naval Research Laboratory

4555 Overlook Ave., SW

Washington, DC 20375

USA

(202) 767-5111

202/767-4050

joe.white@nrl.navy.mil

Ludwig Winkel

Siemens AG

Siemensallee 84

Karlsruhe 76181,

GERMANY

(49) 7215956098

49/7215954938

ludwig.winkel@siemens.com

Steven Wissler

Industrial Ethernet Net. Guide

1322 Marie Ave.

Ephrata, PA 17522

USA

(717) 738-2724

stevew@godfrey.com 
Stan Woods

Agilent Technologies

3500 Deer Creek Road

Palo Alto, CA 94304

USA

(650) 485-5067

650/485-8670

stan_woods@agilent.com

Gary Workman

General Motors CCRW

30300 Mound Road

$\mathrm{M} / \mathrm{C}$ 480-109-267

Warren, MI 48090

USA

(810) 602-9918

586/947-2268

gary.c.workman@gm.com
George Zampetti

Symmetricom

2300 Orchard Parkway

San Jose, CA 95131

USA

(408) 428-7835

925/383-1814

gzampetti@symmetricom.com

Steven Zuponcic

Rockwell Automation

1 Allen-Bradley Drive

Mayfield Hts., OH 44124

USA

(440) 646-5384

440/646-3076

sazuponcic@ra.rockwell.com 


